\title{
The many vacua of gauged extended supergravities
}

\author{
Thomas Fischbacher
}

Received: 17 November 2008 / Accepted: 30 November 2008

(C) Springer Science+Business Media, LLC 2008

\begin{abstract}
A novel method is presented which employs advanced numerical techniques used in the engineering sciences to find and study the properties of nontrivial vacua of gauged extended supergravity models. While this method only produces approximate numerical data rather than analytic results, it overcomes the previous limitation of only being able to find vacua with large residual unbroken gauge symmetry groups. The effectiveness of this method is demonstrated by applying it to the technically most challenging $D \geq 3$ scalar potential-that of $\mathrm{SO}(8) \times \mathrm{SO}(8)$ gauged $\mathcal{N}=16$ Chern-Simons Supergravity in $D=3$. Extensive data on the properties of 99 different vacua (92 of them new) of this model are given. Furthermore, techniques are briefly discussed which should allow using this numerical information as an input to the construction of semi-automatic stringent analytic proofs on the locations and properties of vacua. It hence is argued that these combined techniques presumably are powerful enough to systematically map all the nontrivial vacua of every supergravity model.
\end{abstract}

Keywords Chern-Simons supergravity $\cdot$ Numerical methods $\cdot$ Automatic differentiation · Maximal supergravity · Gauged supergravity · Vacuum solutions

\section{Introduction}

This work utilizes techniques that are widely used in the Engineering disciplines to solve an old open problem in supergravity/superstring theory. While these approaches manage to produce results that have a number of applications considering the present

T. Fischbacher $(\bowtie)$

University of Southampton School of Engineering Sciences,

Highfield Campus, University Road, Southampton SO17 1BJ, UK

e-mail: t.fischbacher@soton.ac.uk 
focus of activity in string theory, the study of supergravity models itself did not receive nearly as much attention recently as it did twenty years ago. As the relevant aspects of the problem can be presented in a self-contained way that requires little more than basic knowledge in (multi)linear algebra, it is expected that parts of the program of mapping all supergravity vacua may be carried out by non-field-theorists. Thus, both a brief gentle introduction to the physical context (which may be skipped by experts) as well as a complete specification of the mathematical construction relevant for the most challenging example- $\mathcal{N}=16, D=3$ Chern-Simons Supergravity with gauge group $S O(8) \times S O(8)$-are given. ${ }^{1}$

\subsection{Motivation and context}

Both quantum mechanics and the special theory of relativity are believed to be based on deep insights into the structure of the physical world. Yet, under very general assumptions, any attempt to reconcile them in an unified framework necessarily leads to relativistic quantum field theory (cf. e.g. [2,35]). The perhaps most striking features of quantum field theory are (i) that it was spectacularly successful in producing quantitative agreement between theory and experiment hitherto unknown in the whole history of physics, (ii) that it is a more rigid construction than one would naively guess and involves a number of 'hidden catches' (such as in the form of anomalies that must cancel) which impose highly nontrivial restrictions which invalidate many constructions which may naïvely seem to make sense, and finally, (iii) that putting it on a sound mathematical foundation still remains a highly elusive problem. Yet, quantum field theory has had some striking successes both in mathematics and in physics. Apart from e.g. providing a framework to both explain the origin of and generalize knot theoretic invariants [36], to effectively compute invariants of four-manifolds [30], and much more, one of the most spectacular breakthroughs was the (experimentally verified) unification of the electromagnetic and weak fundamental forces [34]. While the framework in which this is achieved - nonabelian gauge theory-is powerful enough to also allow further unification of the electroweak with the strong force (in various ways), fundamental obstacles prevent the construction of a fully unified quantum theory of all fundamental forces, including gravity. At the heart of this problem lies the observation that the quanta of gravitational attraction-gravitons - differ in important characteristics from the quanta of the other fundamental forces: while the source currents of the electromagnetic, weak, and strong interaction behave in an analogous way under rotations of space, those of gravity do not. Consequently, a fully unified theory of all forces would have to involve extensions of spacetime symmetry (i.e. the Lorentz group) that allow one to overcome this obstacle. Such extensions do exist, but (due to the strong restrictions imposed by the framework of quantum field theory) involve a generalization of the notion of a symmetry group, which, despite being highly un-intuitive, remarkably seems to be perfectly compatible

\footnotetext{
1 Note that we are not accurate in properly discerning between $\operatorname{Spin}(8)$ and $S O(8)$ here. While this model came to be known as the $S O(8) \times S O(8)$ gauged model, it is clear that the double cover must be meant, for the spacetime scalars transform in spinorial representations.
} 
with the requirements of quantum field theory. These 'supersymmetry' transformations unify particles of different spin, and, as such, ultimately even allow unification of force quanta (bosons of integer spin) with matter quanta (fermions of half-integer spin).

Even temporarily ignoring quantum mechanics, finding supersymmetric extensions of general relativity is an interesting problem in itself. There is a limit on the number of supersymmetry generators possible due to the restriction that increasing their number unifies more and more fundamental fields. This makes the construction ever more rigid until complete unification - in which all fields are related to one another through supersymmetry transformations - is achieved. Then, both the particle content of the model as well as all interactions are completely determined by symmetry requirements, usually up to some discrete choices between alternative possible constructions, and very few (e.g. one) real parameters. One finds that, surprisingly, different constructions of supergravity usually are related to one another by going up and down in the number of spacetime dimensions. In particular, most supergravity models (though not the three-dimensional gauged Chern-Simons models we are mainly concerned with in this work) can be obtained through suitable compactification of a higher-dimensional ancestor model, i.e. splitting $D+1$-dimensional spacetime into $3+1$-dimensional Minkowski space and some $D-3$-dimensional compact space $\mathcal{M}$ and then considering supergravity on such spacetime backgrounds with the 'radius' of $\mathcal{M}$ going to zero. By studying supergravity in various spacetime dimensions, one indeed finds that virtually all models have a single common ancestor, the (classically) unique $10+1$-dimensional supergravity discovered in 1978 by Cremmer et al. [9].

Compactification of eleven-dimensional supergravity to ten spacetime dimensions gives supergravity models which are known to be the low energy limit of tendimensional superstring theory. Therefore, supergravity is intimately linked to superstring theory, and indeed, it is now widely believed that eleven-dimensional supergravity is the low energy limit of an elusive eleven-dimensional theory preliminarily called $M$-Theory [37], which can be reduced to each of the five possible constructions of ten-dimensional superstring theory and in which two-dimensional membranes rather than strings seem to play a fundamental role.

Progress in theoretical physics often comes from advances in our understanding of the role of symmetry principles. It is hence interesting to observe that-rather unexpectedly — new global (and rather special) symmetries (and hence, due the Noether theorem, conserved currents) emerge in compactifications of eleven-dimensional supergravity to lower dimensions. One may conjecture that these can be traced back to hidden symmetry properties of eleven-dimensional $M$-Theory that are not well understood yet, but may eventually become important in the construction of the full theory [3,4,7,10-12].

An important motivation of practical relevance to understand the detailed structure of supergravity models comes from the 'AdS/CFT conjecture' that some models of supergravity have an equivalent dual (in the sense of a one-to-one dictionary of notions and statements) description in terms of conformal field theory in lower-dimensional space [24]. This duality is exciting as it e.g. may allow the computation of quantities which are technically difficult (which may mean: at present impossible) to obtain in a direct approach by taking a detour through the dual theory. 
While a large number of quantum field theories can be constructed that seem to be free of internal inconsistencies, the rules of the game are rather limited due to such constraints as anomaly cancellation, renormalizability, and ghost-freeness. It is therefore not surprising to see that effects well known from the standard model of particle physics also play a role in much more general models whose purpose is more about understanding how quantum field theory works than about explaining the observed world. In particular, the Higgs effect [21]—spontaneous breaking of a local symmetry due to a nonzero background of a charged scalar field-also plays a role in supergravity models with scalars. What one finds here is that a model can have different (perturbatively) stable vacuum solutions that differ by the background values of these scalar fields, and have markedly different properties of elementary particles. One particular vacuum solution that recently attracted considerable interest after 25 years of dormancy is the stationary point in the Higgs potential of maximally supersymmetric four-dimensional supergravity which breaks the gauged R-symmetry group $S O(8)$ down to $S U(3) \times U(1)$ (which-purely coincidentally-also is the local symmetry underlying electromagnetism and the strong force). This solution, originally found by Warner [33], seems to be related via AdS/CFT duality to a $2+1$-dimensional field theory model describing the physics of coincident membranes of $10+1$-dimensional $M$-Theory [5,23], providing another link [1,20] by which detailed information about the properties of supergravity vacua may help to ultimately solve the puzzle of the structure of $M$-Theory.

\subsection{The vacua of gauged extended supergravity}

The existence of scalar field configurations that give stable alternative vacua of models with extended supergravity was first demonstrated in [6] for $S O(5)$-gauged fourdimensional extended supergravity. From the perspective of nontrivial vacuum solutions, the most challenging and most interesting supergravity models are those which-in $D$ dimensions - feature a hidden global $E_{11-D(11-D)}$ symmetry. In $D=3$, this global symmetry group is the (maximal split real form of the) largest exceptional Lie group $E_{8}$. These models may be deformed in such a way that different subgroups of this exceptional global symmetry get promoted to a gauge group. Retaining supersymmetry then mandates the introduction of a potential for the scalar fields. Usually (but not always), setting all scalar fields to zero gives a stable vacuum solution which then has the largest residual unbroken symmetry. Other stationary points, which usually exist (sometimes in large number), are not true extrema but saddle points in the potential, may or may not correspond to stable vacua, and spontaneously break the gauge symmetry to smaller gauge groups.

\section{The scalar potential of gauged $\mathcal{N}=16 D=3$ supergravity}

\subsection{On the choice of the example}

The $\mathrm{SO}(8) \times \mathrm{SO}(8)$ gauged $\mathcal{N}=16 D=3$ model was chosen as the primary example to explain the method for these reasons: 
- From the purely technical perspective, the scalar potential of this model is singled out as the most challenging one in $D \geq 3$ : it lives on the 128-dimensional octooctonionic plane $\mathrm{E}_{8(8)} /\left(\operatorname{Spin}(16) / \mathbb{Z}_{2}\right)$ and does not show any of the simplifications that occur for other gauge groups not contained in $\operatorname{Spin}(16) / \mathbb{Z}_{2} \subset \mathrm{E}_{8(8)}$.

- Despite the complexity of this potential, detailed data (including mass spectra) are available on a number of nontrivial vacua: this allows independent validation of results.

- As the three-dimensional maximal gauged models have been discovered more recently than higher-dimensional ancestors, literature available on them uses more systematized concepts which also generalize to the higher-dimensional cases.

- Folk lore says that vacua in higher-dimensional gauged maximal supergravity models have counterparts in lower dimensions. We therefore naturally expect both the vacuum structure in $D=3$ to be richer than in higher dimensions, as well as to give clues about possible higher-dimensional ancestors of newly discovered vacua.

\subsection{General structure of the problem}

The full Lagrangian of this model is given in [27]. It is not repeated here (although this would be desirable for the sake of completeness) as this would require us to introduce conventions for $3+1$-dimensional spacetime vector and spinor indices which would inevitably clash with our extensive conventions on subgroup index alphabets. In this work, we are (almost) exclusively concerned with the scalar potential $\mathcal{L}_{\text {Vac }}$ as well as a related auxiliary quantity, which (in this context) will be called the 'misalignment' $\mathcal{M}^{I \dot{A}}$ :

$$
\begin{aligned}
\mathcal{L}_{\mathrm{Vac}} /\left(g^{2} e\right) & =\frac{1}{8}\left(A_{1}^{I J} A_{1}^{I J}-\frac{1}{2} A_{2}^{I \dot{A}} A_{2}^{I \dot{A}}\right) \\
\mathcal{M}^{I \dot{A}} & =3 A_{1}^{I M} A_{2}^{M \dot{A}}-A_{2}^{I \dot{B}} A_{3}^{\dot{A} \dot{B}}
\end{aligned}
$$

These expressions are defined on the 128-dimensional octooctonionic plane $\mathcal{P}$ as follows: the matrices $A_{1}, A_{2}, A_{3}$ of respective size $16 \times 16,16 \times 128$, and $128 \times 128$ are specific linear combinations (details to be given further down) of entries of the so-called T-tensor, which in this case is a $248 \times 248$ matrix of the form $T=\mathcal{V}^{T} \Theta \mathcal{V}$, where the (usually sparsely occupied) $248 \times 248$ matrix $\Theta$ is a constant that describes the embedding of the gauge group-here $\mathrm{SO}(8) \times \mathrm{SO}(8)$-into $E_{8(8)}$. $\mathcal{P}$ can be understood as being embedded into the 248-dimensional manifold $E_{8(8)}$ which itself is a subgroup of the double cover of the noncompact real form $\mathrm{SO}(128,120)$ of the group of rotations in 248-dimensions. There is a 1:1 correspondence between points on $\mathcal{P}$ and matrices $\mathcal{V}$ of the form $\mathcal{V}=\exp \left(c^{A} G_{A}^{(128)}\right)$ with $c^{A}$ being an entry of a 128-dimensionalZ vector of coordinates on $\mathcal{P}$ and $G_{A}^{(128)}$ the corresponding $248 \times 248$ generator matrix of $E_{8(8)}$. The potential $\mathcal{L}_{\text {Vac }}$ has a local maximum at the origin, $c^{A}=0$, which retains maximal symmetry. While there seem to be no further true extrema, it is known that there nevertheless are additional stationary (saddle) points which in some cases correspond to meaningful (perturbatively) stable physical backgrounds (vacua). The saddle points $d \mathcal{L} / d c^{A}=0$ can be alternatively characterized by the vanishing of the $16 \times 128$ matrix $\mathcal{M}^{I \dot{A}}$. 


\subsection{Detailed conventions}

For the sake of easy reproducibility of results, conventions are given in full detail. We generally use the Einstein summation convention: any index which occurs twice in a product of tensors is implicitly being summed over. For Spin(N) groups, we do not discern between upper and lower indices.

Conventions for indices denoting different group representations are:

\begin{tabular}{|c|c|}
\hline Indices & Range (representation) \\
\hline$I, J, K, \ldots$ & $1 \ldots 16$ (Spin(16) vector) \\
\hline$[I J],[K L]$, & $1 \ldots 120$ (Spin(120) adjoint) \\
\hline$\overline{A, B}, \overline{C, \ldots}$ & $1 \ldots 128$ (Spin(16) spinor) \\
\hline$\dot{A}, \dot{B}, \dot{C}, \ldots$ & $1 \ldots 128$ (Spin(16) co-spinor) \\
\hline $\mathcal{A}, \mathcal{B}, \mathcal{C}, \ldots$ & $1 \ldots 248\left(E_{8(8)}\right.$ fundamental/adjoint $)$ \\
\hline$i, j, k, \ldots$ & $1 \ldots 8(\operatorname{Spin}(8)$ vector $)$ \\
\hline$\alpha, \beta, \gamma, \ldots$ & $1 \ldots 8$ (Spin(8) spinor) \\
\hline$\dot{\alpha}, \dot{\beta}, \dot{\gamma}, \ldots$ & $1 \ldots 8$ (Spin $(8)$ co-spinor $)$ \\
\hline
\end{tabular}

We generally list only the nonzero components of all tensors. At the heart of the construction lies the Spin(8) Clifford algebra, given as a set of $88 \times 8$ matrices $\gamma^{i}$ that satisfy

$$
\begin{aligned}
& \gamma_{\alpha \dot{\gamma}}^{i} \gamma_{\alpha \dot{\delta}}^{j}+\gamma_{\alpha \dot{\gamma}}^{j} \gamma_{\alpha \dot{\delta}}^{i}=2 \delta^{i j} \delta_{\dot{\gamma} \dot{\delta}} \\
& \gamma_{\alpha \dot{\gamma}}^{i} \gamma_{\beta \dot{\gamma}}^{j}+\gamma_{\alpha \dot{\gamma}}^{j} \gamma_{\beta \dot{\gamma}}^{i}=2 \delta^{i j} \delta_{\alpha \beta} \\
& \gamma_{\alpha \dot{\gamma}}^{i} \gamma_{\beta \dot{\delta}}^{i}+\gamma_{\beta \dot{\gamma}}^{i} \gamma_{\alpha \dot{\delta}}^{i}=2 \delta_{\alpha \beta} \delta_{\dot{\gamma} \dot{\delta}} \\
& \gamma_{\alpha \dot{\gamma}}^{i} \gamma_{\beta \dot{\delta}}^{i}+\gamma_{\alpha \dot{\delta}}^{i} \gamma_{\beta \dot{\gamma}}^{i}=2 \delta_{\alpha \beta} \delta_{\dot{\gamma} \dot{\delta}} \\
& \gamma_{\alpha \dot{\gamma}}^{i} \gamma^{j}{ }_{\alpha \dot{\delta}}+\gamma_{\alpha \dot{\delta}}^{i} \gamma_{\alpha \dot{\gamma}}^{j}=2 \delta^{i j} \delta_{\dot{\gamma} \dot{\delta}} \\
& \gamma_{\alpha \dot{\gamma}}^{i} \gamma_{\beta \dot{\gamma}}^{j}+\gamma_{\beta \dot{\gamma}}^{i} \gamma_{\alpha \dot{\gamma}}^{j}=2 \delta^{i j} \delta_{\alpha \beta}
\end{aligned}
$$

Our detailed conventions on $\gamma_{\alpha \dot{\gamma}}^{i}$ will become important later on, for different reasons. It is possible to satisfy these conditions by $8 \times 8$ matrices which each have one single entry \pm 1 per row and column. This following choice reproduces the conventions given in [17]: we concisely list all 64 nonzero entries $\gamma_{\alpha \dot{\gamma}}^{i}$ in the form $P Q R_{ \pm}$, meaning $\gamma^{i=P}{ }_{\alpha=Q} \dot{\gamma}=R= \pm 1$ :

$$
\begin{aligned}
& 118_{+} 127_{-} 136_{-} 145_{+} 15_{-} 14_{+} 172_{+} 181_{-} \\
& 212_{+} 221_{-} 234_{-} 243_{+} 256_{+} 265_{-} 278_{-} 287_{+} \\
& 315_{+} 326_{-} 337_{+} 348_{-} 351_{-} 362_{+} 373_{-} 384_{+} \\
& 413_{+} 424_{+} 431_{-} 442_{-} 457_{-} 468_{-} 475_{+} 486_{+} \\
& 514_{+} 523_{-} 532_{+} 541_{-} 558_{+} 567_{-} 576_{+} 585_{-} \\
& 616_{+} 625_{+} 638_{+} 647_{+} 652_{-} 661_{-} 674_{-} 683_{-} \\
& 717_{+} 728_{+} 735_{-} 746_{-} 753_{+} 764_{+} 771_{-} 782_{-} \\
& 811_{+} 822_{+} 833_{+} 844_{+} 855_{+} 866_{+} 877_{+} 888_{+}
\end{aligned}
$$


From these eight matrices, we form the real $128 \times 128$ matrices $\Gamma_{A \dot{B}}^{I}$ of the Clifford algebra of 16-dimensional Euclidean space via the embedding $\operatorname{Spin}(8) \times \operatorname{Spin}(8) \subset$ Spin(16). It generally makes sense to define a set of index splitting/embedding matrices for all of which we will use the name $H$ and which are discerned by the types of indices they carry. For these, we use the conventions given below. Here, we encounter an annoying issue that can be traced back to the widespread yet conceptually awkward convention to use indices for a $D$-dimensional representation in the range $1 \ldots D$ rather than $0 \ldots(D-1)$. In order to deal with this, we define $\mathcal{R}(p, q):=$ $(p-1) \cdot 8+(q-1)+1$. Thus, we have:

$$
\begin{aligned}
H_{i}^{I} & =\delta^{I, i} \quad H_{\dot{i}}^{I}=\delta^{I, \bar{i}+8} \\
H_{\alpha \beta}^{A} & =\delta_{A, \mathcal{R}(\alpha, \beta)} \quad H_{\dot{\alpha} \dot{\beta}}^{A}=\delta_{A, 64+\mathcal{R}(\dot{\alpha}, \dot{\beta})} \\
H_{\alpha \dot{\beta}}^{\dot{A}} & =\delta_{\dot{A}, \mathcal{R}(\alpha, \dot{\beta})} \quad H_{\dot{\gamma} \delta}^{\dot{A}}=\delta_{\dot{A}, 64+\mathcal{R}(\dot{\gamma}, \delta)} \\
H_{B}^{\mathcal{A}} & =\delta^{\mathcal{A}, B} \quad H_{[\underline{[I J]}}^{\mathcal{A}}=\delta^{\mathcal{A}, 128+\underline{[I J]}}
\end{aligned}
$$

Additionally, we use the convention

$$
\delta_{k_{1} \ldots k_{n}}^{i_{1} \ldots i_{n}}=(1 / n !) \cdot \sum_{p \in \pi_{n}}(-1)^{\operatorname{sign}(p)} \delta_{k_{p_{1}}}^{i_{1}} \cdots \delta_{k_{p_{n}}}^{i_{n}}
$$

so that this 'generalized Kronecker delta' can be used as an anti-symmetrizing projector.

Then,

$$
\begin{aligned}
\Gamma_{A \dot{A}}^{I}= & H_{i}^{I} H_{\alpha \beta}^{A} H_{\gamma \dot{\delta}}^{\dot{A}} \delta_{\alpha \gamma} \gamma_{\beta \dot{\delta}}^{i}+H_{i}^{I} H_{\dot{\alpha} \dot{\beta}}^{A} H_{\dot{\gamma} \delta}^{\dot{A}} \delta_{\dot{\alpha} \dot{\gamma}} \gamma_{\delta \dot{\beta}}^{i}+H_{\dot{i}}^{I} H_{\alpha \beta}^{A} H_{\dot{\gamma} \delta}^{\dot{A}} \delta_{\beta \delta} \gamma_{\alpha \dot{\gamma}}^{\bar{i}} \\
& -H_{\dot{i}}^{I} H_{\dot{\alpha} \dot{\beta}}^{A} H_{\gamma \dot{\delta}}^{\dot{A}} \delta_{\dot{\beta} \dot{\delta}} \gamma_{\gamma \dot{\alpha}}^{\bar{i}}, \\
\Gamma_{A B}^{I J}= & \delta_{K L}^{I J} \Gamma_{A \dot{C}}^{K} \Gamma_{B \dot{C}}^{L} .
\end{aligned}
$$

Considering efficient numerical computations involving explicit sparse matrix representations, these particular conventions have a number of remarkable highly desirable properties which do not hold in general but will come to play an important role to make numerical computations fast (this is our first important trick!), such as in particular:

$$
\begin{gathered}
\Gamma_{A \dot{C}}^{I} \Gamma_{D \dot{C}}^{J} \Gamma_{D \dot{E}}^{K} \Gamma_{B \dot{E}}^{L} \delta_{M N P Q}^{I J K L}=\Gamma_{A \dot{C}}^{M} \Gamma_{D \dot{C}}^{N} \Gamma^{P}{ }_{D \dot{E}} \Gamma^{Q}{ }_{B \dot{E}} \\
\Gamma_{A \dot{C}}^{I} \Gamma^{J}{ }_{D \dot{C}} \Gamma^{K}{ }_{D \dot{E}} \delta_{M N P}^{I J K}=\Gamma^{M}{ }_{A \dot{C}} \Gamma^{N}{ }_{D \dot{C}} \Gamma^{P}{ }_{D \dot{E}} .
\end{gathered}
$$

As usual, we further define:

$$
\Gamma_{A B}^{K L}:=\delta_{I J}^{K L} \Gamma_{A \dot{C}}^{I} \Gamma^{J}{ }_{B \dot{C}}
$$


Before we can proceed to define the 248 sparse $248 \times 248$ matrices $\left(G_{\mathcal{A}}^{(E 8)}\right)^{\mathcal{C}}{ }_{\mathcal{B}}$ (the generators of the group $\left.E_{8(+8)}\right)$, we need further conventions that extract the adjoint index $[I J]$ from a pair of vector indices $I, J$. We also use the name $H$ for this form of index projection, in detail:

$$
\begin{aligned}
H_{K L}^{[I J]}= & \delta_{M=[I J]}, M=(K-1) \cdot 16+L-K(K+1) / 2 \\
& -\delta_{M=\underline{[I J]}, M=(L-1) \cdot 16+K-L(L+1) / 2}
\end{aligned}
$$

and likewise for $H_{[I J]}^{K L}$. Also, we define $H_{I J}^{\mathcal{A}}:=H_{[I J]}^{\mathcal{A}} H_{I J}^{[I J]}$.

With these, we first introduce generators for the group Spin(16) of rotations in 16 dimensions, in the adjoint representation:

$$
\left(G_{\underline{[I J]}}^{(\operatorname{Spin}(16))}\right)^{\underline{[K L]}} \underline{\underline{[M N]}}=-H_{\underline{[I J]}}^{I J} H_{K L}^{[K L]} H_{[\underline{[M N]}}^{M N} \delta_{I^{\prime} J^{\prime}}^{I J} \delta_{K^{\prime} L^{\prime}}^{K L} \delta^{I^{\prime} K^{\prime}} \delta_{L^{\prime} J^{\prime}}^{M N}
$$

Then, the $\left(G_{\mathcal{A}}^{(E 8)}\right)^{\mathcal{C}} \mathcal{B}$ are given by:

$$
\begin{aligned}
\left(G_{\mathcal{A}}^{(E 8)}\right)^{\mathcal{C}}{ }_{\mathcal{B}}= & H_{\underline{[I J]}}^{\mathcal{A}} H_{\underline{[K L]}}^{\mathcal{B}} H_{\mathcal{C}}^{[M N]}\left(G_{\underline{[I J]}}^{(\operatorname{Spin}(16))}\right)^{\underline{[K L]}} \underline{\underline{[M N]}} \\
& +\frac{1}{4} \Gamma_{A B}^{I J}\left(H_{I J}^{\mathcal{A}} H_{A}^{\mathcal{B}} H_{\mathcal{C}}^{B}+H_{B}^{\mathcal{A}} H_{I J}^{\mathcal{B}} H_{\mathcal{C}}^{A}-H_{A}^{\mathcal{A}} H_{B}^{\mathcal{B}} H_{\mathcal{C}}^{I J}\right)
\end{aligned}
$$

Finally, we need the gauge group embedding tensor $\Theta_{\mathcal{A B}}$, which for the particular case $\mathrm{SO}(8) \times \mathrm{SO}(8)$ has the form:

$$
\Theta_{\mathcal{M N}}=H^{\left[\frac{[I J]}{\mathcal{M}}\right.} H_{\overline{\mathcal{N}}}^{[K L]} H_{[I J]}^{I J} H_{[K L]}^{K L}\left(H_{I}^{\bar{i}} H_{J}^{\bar{j}} H_{K}^{\bar{k}} H_{L}^{\bar{l}} \delta_{\bar{i} \bar{j}}^{\bar{k} \bar{l}}-H_{I}^{i} H_{J}^{j} H_{K}^{k} H_{L}^{l} \delta_{i j}^{k l}\right)
$$

Due to the special role of vector/scalar duality in three dimensions, the constraints on possible gauge groups are less stringent than in higher-dimensional maximal supergravity: Any subgroup $H \subset E_{8(+8)}$ for which the corresponding embedding tensor does not carry a contribution in the 27000-dimensional irreducible representation of $E_{8}$ can be chosen as gauge group. Apart from the non-compact forms $(\mathrm{SO}(\mathrm{p}, 8-\mathrm{p}) \times \mathrm{SO}(\mathrm{p}, 8-\mathrm{p})$ for $1 \leq p \leq 7)$, which have analoga in higher dimensions such as $\mathrm{SO}(\mathrm{p}, 8-\mathrm{p})$ gauged $\mathcal{N}=8$ supergravity in $D=4$, there are a number of additional possibilities here that include exceptional gauge groups [27], such as e.g. $G_{2(2)} \times F_{4(4)}$ or $E_{7(-5)} \times S U(2)$.

Given the $\left(G_{\mathcal{A}}^{(E 8)}\right)^{\mathcal{C}}{ }_{\mathcal{B}}$, the explicit expressions for the matrices $A_{1}, A_{2}, A_{3}$ as functions of 128 coordinates on the octooctonionic plane $c^{A}$ are: 


$$
\begin{aligned}
& \mathcal{V}^{\mathcal{M}}{ }_{\mathcal{A}}=\exp \left(c^{A} H_{A}^{\mathcal{A}} G_{\mathcal{A}}^{(E 8)}\right)^{\mathcal{M}} \mathcal{A} \\
& T_{\mathcal{A B}}=\mathcal{V}^{\mathcal{M}}{ }_{\mathcal{A}} \Theta_{\mathcal{M N}} \mathcal{V}^{\mathcal{N}}{ }_{\mathcal{B}} \\
& \eta^{\mathcal{A B}}=H_{A}^{\mathcal{A}} H_{B}^{\mathcal{B}} \delta A B-H_{\underline{[I J]}}^{\mathcal{A}} H_{[K L]}^{\mathcal{B}} \delta^{\left[\frac{[I J]}{[K L]}\right.} \\
& \theta=\frac{1}{248} \eta^{\mathcal{M N}} \Theta_{\mathcal{M N}} \\
& A_{1}^{I J}=\frac{8}{7} \theta \delta_{I J}+\frac{1}{7} T_{\mathcal{A B}} H_{\underline{[I K]}}^{\mathcal{A}} H_{[\underline{[J]}]}^{\mathcal{B}} H_{I K}^{[I K]} H_{J K}^{[J K]} \\
& A_{2}^{I \dot{A}}=-\frac{1}{7} T_{\mathcal{A B}} H_{\underline{[I J]}}^{\mathcal{A}} H_{I J}^{[I J]} H_{A}^{\mathcal{B}} \Gamma_{A \dot{A}}^{J} \\
& A_{3}^{\dot{A} \dot{B}}=2 \theta \delta_{\dot{A} \dot{B}}+\frac{1}{48} \Gamma_{\dot{A} \dot{B}}^{I J K L} T_{\mathcal{A B}} H_{[\underline{[I J]}}^{\mathcal{A}} H_{[K L]}^{\mathcal{B}} H_{I J}^{\frac{[I J]}{I J}} H_{K L}^{[K L]}
\end{aligned}
$$

Finally the scalar potential is then given as:

$$
\mathcal{L}_{\mathrm{Vac}} /\left(g^{2} e\right)=\frac{1}{8}\left(A_{1}^{I J} A_{1}^{I J}-\frac{1}{2} A_{2}^{I \dot{A}} A_{2}^{I \dot{A}}\right)
$$

\section{The method}

Previous approaches towards finding stationary points of supergravity potentials on high-dimensional scalar manifolds involved analytic treatments based on a restriction of the full problem to low-dimensional submanifolds invariant under some (usually large) chosen subgroup of the gauge group (e.g. [22,32,33]). While this is a powerful approach, especially in conjunction with symbolic algebra tailored to the task [13], a fundamental problem is the inability to find stationary points which do not lie on any low-dimensional submanifold accessible in such a way. Hence, a complementary numerical approach that is able to yield information about otherwise inaccessible solutions is highly desirable.

One fundamental objection against the idea of tackling the problem numerically is that stationary points are not isolated but lie on a manifold that is invariant under rotations in the gauge group, hence we should expect to obtain their position in general form, which is a mostly meaningless collection of coordinates. There are, however, simple numerical techniques to address this problem and ensure that stationary points are automatically rotated into a useful form. This will be explained at the end of this section.

The basic ingredients of the numerical approach are:

1. A real-valued function $P$ that can be evaluated fast to high accuracy which provides information about how much a given point violates the stationarity condition. [Using the misalignment tensor $\mathcal{M}$, this requires fast computation of the tensor $A_{3}$, made possible by Eq. (7).] 
2. A fast method to evaluate the gradient of $P$.

3. An efficient numerical algorithm to find minima of $P$ in constrained space. (Practically all such algorithms utilize the gradient of $P$.)

4. Sufficient numerical precision to allow the extraction of useful data.

5. Post-processing of raw data to obtain information about particle mass spectra.

6. A reduction step that brings a generic point on the submanifold of equivalent vacua to a simple coordinate representation by applying a suitable gauge group rotation.

\subsection{The quasipotential $P$}

As it stands, the potential $\mathcal{L}_{\text {vac }}$ is not well suited for the purpose of performing a numerical search for stationary points, all of which (except for the trivial one at the origin) are saddle points. Therefore, we instead base our search on the function:

$$
\tilde{P}=\mathcal{M}_{I \dot{A}} \mathcal{M}_{I \dot{A}}
$$

Here, we have $\tilde{P} \geq 0$ and $\tilde{P}=0$ precisely for the stationary points of $\mathcal{L}_{\text {vac }}$. While it often is not a good idea to recast the problem of numerically solving an equation as an optimization problem of the form $([\mathrm{LHS}]-[\mathrm{RHS}])^{2}=$ min!, this works sufficiently well for our purposes here. However, as it stands the function $\tilde{P}$ also is not very well suited for numerical optimization, as the degree of violation of the stationarity condition measured by $\tilde{P}$ depends roughly exponentially on the coordinate distance of the point in the octooctonionic plane from the origin. Consequently, optimization algorithms that iteratively try to reduce the objective function will encounter extremely steep gradients close to extremely narrow 'golf hole style' solutions, which causes the optimizer to 'wildly bounce around' in the allowed parameter regime. While it is precisely this behaviour that may occasionally succeed in producing solutions that are otherwise unlikely to arise, one would nevertheless generally prefer a somewhat more well-behaved function. For the analysis presented here, the choice was

$$
P=(\operatorname{Arsinh} \tilde{P})\left(c^{A} c^{A}\right)^{\rho}
$$

Without the extra factor radius $^{2 \rho}$, the inverse hyperbolic sine of the exponential would give a potential which for generic configurations is roughly linear in the distance from the origin - up to extra dips corresponding to nontrivial solutions. However, the 'funnel-like' shape would make the region around the origin so attractive that it becomes rather difficult to find solutions lying far out. Setting the constant $\rho$ to about -0.5 turns the funnel into a (more or less) smooth plateau with a very broad minimum. By changing this parameter, one can tune the optimization algorithm to spend more time in regions of varying distance from the origin, and hence systematically search for solutions lying in a certain distance region.

\subsection{Fast gradients through sensitivity analysis}

At about the same time when N. Warner worked on the structure of the four-dimensional cousin of the model studied here [32,33] in Supergravity, Speelpenning-working in 
computer science-presented an algorithmic approach that allows the fast evaluation of gradients for scalar functions of many parameters [31] that also can be used to great effect for the analysis of supergravity potentials. Essentially, he showed that, given a differentiable real-valued function $f: \mathbb{R}^{n} \rightarrow \mathbb{R}$ of $n$ parameters, then a computer program that computes the value of $f$ at a specific point $P$ within time $T$, can be mechanically translated to a computer program that computes to high accuracy the $n$ entries of $\nabla f$ at $P$ within a time bounded by 5T, regardless of the number of parameters $n$ !

This evidently is far more powerful than the naive approach to compute the gradient from finite differences, which would require at least a computation time of $(n+1) \cdot T$ and not produce highly accurate results.

The price that has to be paid when using Speelpenning's method is that (i) all intermediate results in the computation have to be retained (rather than overwritten) as $f$ is evaluated, (ii) for each intermediate numerical quantity, memory space has to be reserved to hold another number, and (iii) at the end of the first stage of the 'fast gradient' algorithm, which is the computation of $f$ at the given point, the sequence of operations that were executed to produce this number must be known.

The third requirement means that, for computations that involve decisions on the magnitude of intermediate quantities (e.g. a number of iterations depending on when a certain tolerance level is reached), information on how these decisions were made during the computation has to be retained. Therefore, automated program transformers that map computer code for $f$ to fast computer code for $\nabla f$ produce code which records an execution 'tape' data structure that contains information on all these decisions in the computation of $f$. If the computation of $f$ is structurally simple enough - as is fortunately the case with supergravity potentials and the quasipotentials introduced to measure deviation from the stationarity condition-then it is often advantageous not to use an automated program transformer (such as e.g. ADOLC [19]), but to instead manually re-write the code in such a way that it also offers fast gradients, as this allows tighter code and easily avoids the (slightly awkward) 'tape' data structure.

Briefly stated, the idea underlying the fast computation of the gradient of $f$ at the point $X=\left(x_{1}, \ldots, x_{n}\right)$ is to first compute $z=f(X)$ and associate with every intermediate quantity $y$ an extra quantity $\bar{y}$, which gives $\delta z / \delta y$, i.e. the change of the final result relative to an infinitesimal change of this intermediate value $y$ : if, after the determination of $y$, the computation continued not with this value $y$, but instead with $y+\delta y$, how much would this change the result $z$ ? Starting with the final computation that produced $z$, we can then use the chain rule to backpropagate sensitivities to earlier and earlier intermediate results. Treating input parameters $x_{k}$ in the same way as the intermediate quantities by also associating output sensitivities $\bar{x}_{k}=\delta z / \delta x_{k}$ to these, we eventually obtain the rates of change of the output with each of the inputs, which is just the gradient. The backpropagation algorithm hence works as follows:

1. For every input and intermediate quantity, and also for the result, allocate enough memory to hold two floatingpoint numbers. Initialize all of them to 0.0 .

2. Load the input quantity value cells $x_{n}$ with the input parameters. 
3. Perform the computation of $f$, remembering sufficient information to later retrace in reverse all numerical operations performed. This gives the result $z$. None of the cells holding associated sensitivities are touched.

4. Set the content of the memory cell holding the associated sensitivity $\bar{z}$ of the result $z$ to $1.0 .(\delta z / \delta z=1)$.

5. Retrace all numerical computation steps in reverse. At each step, the output sensitivity of its computational result $y_{m}$ is known: $\bar{y}_{m}=\delta z / \delta y_{m}$. Update the sensitivities of the arguments of the present step that produced $y_{m}$ according to these rules:

- if it is an addition $y_{m}=y_{a}+a_{b}$, then increase the number stored in the cell holding $\bar{y}_{a}$ by $\bar{y}_{m}$, and also increase the number stored in the cell holding $\bar{y}_{b}$ by $\bar{y}_{m}$.

- if it is a multiplication $y_{m}=y_{a} \cdot y_{b}$, then increase the number stored in the cell holding $\bar{y}_{a}$ by $\bar{y}_{m} \cdot y_{b}$, and also increase the number stored in the cell holding $\bar{y}_{b}$ by $\bar{y}_{m} \cdot y_{a}$.

- if it is a function application $y_{m}=g\left(y_{k}\right)$, then increase the number stored in the cell holding $\bar{y}_{k}$ by $\bar{y}_{m} \cdot\left(\partial g / \partial y_{k}\right)\left(y_{k}\right)$.

If one of the summands(/factors) in an addition(/multiplication) operation is a constant, then this does not come with an associated sensitivity, hence we omit the corresponding update step: we are not interested in how the result would change if we changed the fixed constants in the computational algorithm. Note that this situation is covered by the 'function application' rule: if e.g. $y_{20}=5 \cdot y_{17}$, then we can take $g=(x \mapsto 5 \cdot x)$, and hence have to update $\bar{y}_{17}$ by increasing it by $5 \cdot y_{20}$. The 'function application' rule also handles divisions, as we can regard the fraction $p / q$ as the product $p \cdot h(q)$ with $h=(x \mapsto 1 / x)$. When manually re-writing existing code for backpropagation, it is occasionally useful to put in modifications that allow a comparison between an individual intermediate backpropagated sensitivity and the change of the result produced by redoing the computation with an artificially introduced small change to the corresponding intermediate quantity. This allows easy validation of the new code and simplifies locating bugs.

It may be useful to convince oneself how backpropagation works by studying a simple example that is structurally similar to the computation of a supergravity potential, such as

$$
v=\exp x, \quad t=v^{2}, \quad a=1 / 7 \cdot t, \quad z=\left(\operatorname{Arsinh} a^{2}\right) \cdot\left(x^{2}\right)^{0.4}
$$

A more detailed description of backpropagation can be found e.g. in [18]. Considering the associated semantic aspects, an interesting question is what would have to be done in order to extend a given programming language with a 'backpropagation operator' that maps a function to a fast gradient function and allows nesting. This has been studied in [28].

It makes sense to implement the functions to compute the supergravity potential, as well as the quasipotential and its gradient, in the $\mathrm{C}$ programming language, and then interface this to the high-level scripting language Python in order to do the optimizing part of the problem with a readily existing efficient numerical optimizing library 
routine. The Python programming language is an attractive choice here, as a number of useful libraries for tasks such as numerical optimization are readily available due to its high popularity in the Engineering Sciences. In this work, we use the scipy • optimize.fmin_1_bfgs_b function [8] from the 'Scientific Python' package [29].

\subsection{Other ingredients}

A number of minor additional technicalities are used in the fast numerical determination of supergravity vacua:

\subsubsection{Gamma matrices}

The computationally relevant $\operatorname{Spin}(16)$ Gamma matrices $\Gamma^{I}, \Gamma^{I J}, \Gamma^{I J K}, \Gamma^{I J K L}$ all share the property that they are real $128 \times 128$ matrices that have a single nonzero entry per row and per column which is \pm 1 . Due to (7), they allow a memory-efficient compact code representation that stores each of the $\Gamma^{I}$ as a string of 128 signed 8-bit characters where the $k$ th entry gives both the sign ( 1 bit) as well as the index ( 7 bit) of the single nonzero entry in the $k$ th column of $\Gamma^{I}$. As we also need the transpose of $\Gamma^{I}$, this amounts to a total memory requirement of $16 \times 128 \times 2=4096$ byte, as well as some simple command logic to string subsequent lookups and keep track of the sign when dealing with antisymmetrized higher powers of $\Gamma$ matrices. These operations are fast, as they easily fit into the CPU's L1 caches.

\subsubsection{Fast matrix exponentiation}

The sensitivity backpropagation techniques strongly favor a simple algorithm that is reasonably fast to numerically compute $E_{8}$ group elements by exponentiating $E_{8}$ generators. According to the overview paper [26], the method which seemingly also was used in early versions of MATLAB [25] suggests itself here: in order to compute $\exp M$, we first compute $Q=2^{-n} \cdot M$ for $n$ sufficiently large to make the numerical computation of $\exp Q$ via the Taylor series expansion reasonably fast (i.e. $\|Q\|_{2}<1$ ), and then square the result $n$ times, using $\exp (A)^{2}=\exp (2 A)$.

\subsubsection{Search parameters: initial point and the parameter $\rho$}

As the numerical approach presented here only allows 'fishing' for vacua, the question arises how to effectively choose parameters to obtain a large number of different vacua. Considering the structure of the potential, one could start by choosing all 128 spinor components as equidistributed points within a $[-A ; A]^{128}$ cube. This, however, enormously disfavors points close to hyperface midpoints relative to points close to hyperdiagonals. A 'fairer' strategy to choose an initial point seems to be to first choose a random point on the circle $S^{2}$ and then use its coordinates as relative weights for two random points on $S^{64}$. While this approach is indeed viable and produces a number of nontrivial solutions, it must be pointed out that one should not resort to a single strategy here. In particular, most of the stationary points at a large distance from the 
origin which are presented here were found by (i) using equidistribution on an 128dimensional cube for the initial point, and (ii) removing both the exponent $\rho$ as well as using the quasipotential function $\tilde{P}$ instead of $P$.

\subsubsection{Mass spectra}

When numerically determining matrix eigenvalues to obtain particle mass spectra, having easy access to a powerful eigenvalue function from a well-maintained software library is very helpful. The 'Scientific Python' [29] package (which for many purposes can be regarded as a viable alternative to MATLAB) provides the scipy. linalg.eig function that can be put to good use here.

\subsubsection{Beautifying the result}

If the numerical search for a vacuum succeeds, it will return a stationary point which differs from what one would recognize as a 'nice' presentation of the vacuum by an arbitrary rotation in the gauge group. So, the question arises how 'wild' numbers produced by numerical optimization that describe a vacuum can be used to arrive at a 'tame' description. One simple strategy is to again use the same numerical tricks and techniques employed to find the vacuum in the first place (i.e. sensitivity analysis, fast matrix exponentiation, efficient numerical optimization, etc.) in order to find a linear combination of the generators of the gauge group which, after embedding in $E_{8}$ and exponentiation to obtain the corresponding rotation, manages to minimize the objective function of the 128-dimensional coefficient vector $c$

$$
F(c)=\sum_{A=1}^{128}-\left(c^{A}\right)^{4} .
$$

The (very simple) idea here is that, while $\cos ^{2} \phi+\sin ^{2} \phi$ is rotationally invariant, the quantity $\cos ^{4} \phi+\sin ^{4} \phi$ takes on a maximum when $\phi$ is a rotation that aligns the coordinate axes with themselves. So, this optimization favors setting as many vector entries to zero as possible by maximizing entries which already were large in the beginning. This solves the problem sufficiently well for our purposes, but the strategy may easily be refined to instead maximize e.g. the coefficients of the $N$-forms contained in the Spinor $\otimes$ Spinor representation of the diagonal $S O(8)$ subgroup of the gauge group, or similar constructions.

\section{New results}

The code that has been used to identify all the new vacua presented in this work can be obtained by downloading the source code archive of this paper from arXiv. org. ${ }^{2}$

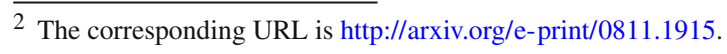


The high-level Python script e8_vacuum from this source can find, beautify, analyze, and automatically typeset data describing nontrivial stationary points in the scalar potential. As the method is not intrinsically exhaustive, but will only produce a 'random' solution (or-occasionally_not converge at all), the following list of program-generated solutions is expected not to be complete even over the limited range investigated. Some of the vacua already were known from earlier work by the author and are listed again for cross-validation purposes of the numerical approach against symbolic group-theory based methods.

In these tables, the (approximate) 'length' of the $\operatorname{Spin}(16)$ spinor $R=\left(c^{A} c^{A}\right)^{1 / 2}$ is given together with the (approximate) value $\Lambda$ of the potential at the stationary point, the number of unbroken (left and right) supersymmetry generators, as well as the dimension of the residual unbroken gauge group $H \subset G=S O(8) \times S O(8)$. The locations of these vacua are given by listing the nonzero coefficients of the 128 dimensional spinor as a set of contributions $\{$ coefficient $\}[\alpha \beta]$, resp. \{coefficient $\}[\dot{\alpha} \dot{\beta}]$, giving $S O(8) \times S O(8)$ spinor/co-spinor indices. The distance $\Delta$ in 128-dimensional Euclidean space of the actual numerically found stationary point and the position given by the coefficients listed is also indicated. Furthermore, mass matrix eigenvalues are listed (as in [14]), with multiplicities given in parentheses where they are greater than 1.

The data given contain detailed numerical mass matrix information about those seven stationary points that were known previously, plus many new ones, but do not claim to be exhaustive. Also, it is conceivable (although improbable) that some numerically obtained stationary point turns out not to correspond to a true exact solution, but only come close to satisfying the stationarity constraint. Therefore, the data presented here may be useful as a first step towards establishing the existence of an analytic expression for the location and properties of a particular conjectured vacuum. In principle, the code used here could be modified with reasonable effort to do computations with high-precision floatingpoint numbers (long double, or even the GNU Multiprecision Arithmetics library [16]). As the exponentials of the spinor coefficients occurring in all analytically known vacua seem to be algebraic numbers, and as there are simple automatized methods to guess low-rank polynomials with integer coefficients given a root known to very high numerical accuracy, going from the numerical data given here to analytic expressions can be made a semi-automatic process. ${ }^{3}$ It must be emphasized that a numerical approach seems to be the only feasible way to learn about the properties of those vacua without any residual gauge symmetry. One may speculate that these vacua should still possess nontrivial discrete symmetries, which may also allow interesting interpolating solutions.

Due to the choice to perform all computations in double-precision floatingpoint, rather limited computational effort invested in the determination of each stationary point, and error accumulation, some of the numerical data presented in the following tables may be slightly inaccurate. The most important limitation presumably is that

\footnotetext{
3 The code provided with the LaTeX source of the arXiv preprint of this work contains a simplistic LISP function that fulfils precisely this purpose. While this function manages to successfully find the polynomial underlying the algebraic expression of the spinor coordinates for the $G_{2} \times G_{2}$ vacuum, ordinary IEEE754 double-float numerical accuracy does not contain enough information to determine with high probability the polynomials underlying more complicated solutions. This hence would require upgrading existing code to work with multiprecision floatingpoint arithmetics.
} 
the given dimension of the residual unbroken gauge group $H$ can only be trusted for stationary points 'close to the origin': for solutions far out (with large negative cosmological constant $\Lambda$ ), this should only be considered a lower bound on the true dimension. In particular, the tables show some entries where multiplicities of eigenvalues of the spinorial mass matrices $A_{1}$ and $A_{3}$ would suggest a nontrivial residual gauge group while $\operatorname{dim}(H)$ is listed at zero. For many (but not all) vacua, the eigenvalues of $A_{1}$ should be symmetric around zero, which may serve as a first nontrivial check on the quality of the numerical data. One should remember that even if the data for any given vacuum should be slightly off, there is a very high chance that a true stationary point is very close to the point specified, whose properties may be determined to high accuracy by restarting the search from the position given with increased numerical precision.

It should be pointed out that, while extensive searches produced most of the stationary points with $R<2.5$ many times over, the solution with $S U$ (4) symmetry at $R \approx 2.167$ which already was known before had to be added manually to the search space: this stationary point seems to be somewhat non-attractive in a random search. One may speculate that this might be due to a contribution in the $(\alpha, \beta)$-spinorial components proportional to the identity, which could be difficult to produce in a random search. Hence it is conceivable that the approach used here systematically misses a number of solutions with large residual symmetry groups that could be obtained by modifying the way the starting point for the search is chosen.

Previously known data about the vacuum structure of the model studied here roughly corresponds to the first page of this lengthy list. All the other data are novel. Some new features exhibited by these stationary points that were not encountered so far include total breaking of all gauge symmetry, as well as $S O(8) \times S O(8)$-asymmetric breaking of supersymmetry. Considering the sheer amount of new data, the question arises how many different stationary points there are in this supergravity potential. Judging from the number of duplicates produced by numerically 'fishing for solutions', the total number that can be found with these methods presumably is large, but not mind-boggingly so. In particular, given this list, it already becomes somewhat difficult (though not impossible) to find further new solutions with $R<2.5$. The author's present estimate is that he certainly would accept a 10:1 bet that the total number is considerably smaller than 10 million. Quite likely, it is even considerably smaller than 10,000. In particular, the total number of solutions with some amount of residual supersymmetry seems to be very small, presumably there are fewer than 25 in total.

\begin{tabular}{|ll|}
\hline$R=0.000 \quad \Lambda=-16.000 \quad$ SUSY $=(8,8) \quad \operatorname{dim}(H)=56$ \\
\hline$\Psi_{128} \quad 0 ; \Delta=0.000000$ \\
\hline $\mathcal{M}_{s} / g^{2}-12.000(\times 128)$ \\
\hline $\mathcal{M}_{v} / g \quad 0.000(\times 128)$ \\
\hline$A_{1} \quad-2.000(\times 8), 2.000(\times 8)$ \\
\hline$A_{3} \quad 0.000(\times 128)$ \\
\hline
\end{tabular}




\begin{tabular}{|c|c|}
\hline \multicolumn{2}{|c|}{$R=1.317 \quad \Lambda=-25.000 \quad \operatorname{SUSY}=(0,0) \quad \operatorname{dim}(H)=42$} \\
\hline$\Psi_{128}$ & $1.317[\dot{3} \dot{4}] ; \Delta=0.000042$ \\
\hline $\mathcal{M}_{s} / g^{2}$ & $-24.000(\times 49),-9.000(\times 64), 0.000(\times 14), 96.000$ \\
\hline $\mathcal{M}_{v} / g$ & $-6.000(\times 7), 0.000(\times 114), 6.000(\times 7)$ \\
\hline$A_{1}$ & $-3.500(\times 8), 3.500(\times 8)$ \\
\hline$A_{3}$ & $-10.500(\times 8),-1.500(\times 56), 1.500(\times 56), 10.500(\times 8)$ \\
\hline
\end{tabular}

\begin{tabular}{|c|c|}
\hline \multicolumn{2}{|c|}{$R=1.491 \quad \Lambda=-28.444 \quad \operatorname{SUSY}=(1,1) \quad \operatorname{dim}(H)=28$} \\
\hline$\Psi_{128}$ & $1.054[83] ; \Delta=0.000416$ \\
\hline $\mathcal{M}_{s} / g^{2}$ & $-26.667(\times 49),-12.444(\times 49), 0.000(\times 28), 16.000,115.556$ \\
\hline $\mathcal{M}_{v} / g$ & $-6.667(\times 7),-1.333(\times 7), 0.000(\times 100), 1.333(\times 7), 6.667(\times 7)$ \\
\hline$A_{1}$ & $-4.000(\times 7),-2.667,2.667,4.000(\times 7)$ \\
\hline$A_{3}$ & $\begin{array}{l}-12.000(\times 7),-9.333,-4.000(\times 7),-1.333(\times 49), 1.333(\times 49), 4.000(\times 7), 9.333 \\
12.000(\times 7)\end{array}$ \\
\hline
\end{tabular}

\begin{tabular}{|l|}
\hline$R=1.763 \quad \Lambda=-36.000 \quad \operatorname{SUSY}=(2,2) \operatorname{dim}(H)=18$ \\
\hline$\Psi_{128} 0.881[24], 0.881[72] ; \Delta=0.000748$ \\
\hline $\mathcal{M}_{s} / g^{2}-32.000(\times 49),-20.000(\times 36), 0.000(\times 38), 28.000(\times 4), 160.001$ \\
\hline $\mathcal{M}_{v} / g \quad-8.000(\times 7),-2.000(\times 12), 0.000(\times 90), 2.000(\times 12), 8.000(\times 7)$ \\
\hline$A_{1}-5.000(\times 6),-3.000(\times 2), 3.000(\times 2), 5.000(\times 6)$ \\
\hline$A_{3}-15.000(\times 6),-11.000(\times 2),-5.000(\times 14),-1.000(\times 42), 1.000(\times 42), 5.000(\times 14)$, \\
\hline
\end{tabular}

\begin{tabular}{|c|c|}
\hline \multicolumn{2}{|c|}{$R=2.030 \quad \Lambda=-45.753 \quad \operatorname{SUSY}=(0,0) \quad \operatorname{dim}(H)=8$} \\
\hline$\Psi_{128}$ & $0.459[66], 0.014[\dot{2} \dot{3}], 1.158[\dot{3} \dot{1}], 0.014[\dot{4} \dot{2}] ; \Delta=0.000443$ \\
\hline $\mathcal{M}_{s} / g^{2}$ & $\begin{array}{l}-53.352(\times 5), \quad-48.309(\times 4), \quad-47.454(\times 8),-38.084(\times 16), \quad-31.009(\times 8), \\
-27.231(\times 2),-23.007(\times 16),-18.154(\times 12),-2.393(\times 2), 0.000(\times 49), 83.506, \\
87.608(\times 2), 145.337(\times 2), 197.167\end{array}$ \\
\hline $\mathcal{M}_{v} / g$ & $\begin{array}{l}-10.661(\times 3),-8.308(\times 8),-4.159(\times 2),-0.925(\times 2),-0.769(\times 8), 0.000(\times 82), \\
0.769(\times 8), 0.925(\times 2), 4.159(\times 2), 8.308(\times 8), 10.661(\times 3)\end{array}$ \\
\hline$A_{1}$ & $-6.539(\times 4),-4.900(\times 4), 4.900(\times 4), 6.539(\times 4)$ \\
\hline$A_{3}$ & $\begin{array}{l}-19.616(\times 4),-14.699(\times 4),-12.631(\times 4),-8.698(\times 4),-5.463(\times 4),-4.539(\times 4), \\
-3.614(\times 4),-3.000(\times 8),-2.900(\times 4),-1.361(\times 16),-0.831(\times 4),-0.379(\times 4), \\
0.379(\times 4), 0.831(\times 4), 1.361(\times 16), 2.900(\times 4), 3.000(\times 8), 3.614(\times 4), 4.539(\times 4), \\
5.463(\times 4), 8.698(\times 4), 12.631(\times 4), 14.699(\times 4), 19.616(\times 4)\end{array}$ \\
\hline
\end{tabular}

\begin{tabular}{|c|c|c|c|}
\hline \multicolumn{4}{|c|}{$R=2.122 \quad \Lambda=-49.821 \quad \operatorname{SUSY}=(0,0) \quad \operatorname{dim}(H)=10$} \\
\hline$\Psi_{128}$ & $0.462[22], 0.462[63], 0.462[86] ; \Delta=0.001035$ & & \\
\hline $\mathcal{M}_{s} / g^{2}$ & $\begin{array}{l}-65.923, \quad-56.599(\times 12), \quad-46.978(\times 16), \\
-24.861(\times 2), \quad-20.365(\times 12), \quad-17.489(\times 4) \\
61.095(\times 8), 210.857,220.146(\times 2)\end{array}$ & $\begin{array}{r}-39.397(\times 6) \\
-6.248(\times 12)\end{array}$ & $\begin{array}{r}-28.652(\times 6) \\
0.000(\times 46)\end{array}$ \\
\hline
\end{tabular}




\begin{tabular}{|lll|}
\hline $\mathcal{M}_{v} / g$ & $-11.557,-10.287(\times 6), \quad-8.287(\times 6), \quad-0.457(\times 6), 0.000(\times 90), 0.457(\times 6)$, \\
& $8.287(\times 6), 10.287(\times 6), 11.557$ \\
\hline$A_{1}$ & $-7.215(\times 2),-5.987(\times 6), 5.987(\times 6), 7.215(\times 2)$ \\
\hline & $-21.646(\times 2), \quad-17.960(\times 6), \quad-13.359(\times 6), \quad-10.588(\times 2), \quad-4.758(\times 12)$, \\
& $-3.843(\times 2),-3.529(\times 16),-2.614(\times 6),-1.386(\times 6),-1.072(\times 6), 1.072(\times 6)$, \\
$A_{3}$ & $1.386(\times 6), \quad 2.614(\times 6), \quad 3.529(\times 16), \quad 3.843(\times 2), \quad 4.758(\times 12), \quad 10.588(\times 2)$, \\
& $13.359(\times 6), 17.960(\times 6), 21.646(\times 2)$
\end{tabular}

\begin{tabular}{|c|c|}
\hline \multicolumn{2}{|c|}{$R=2.167 \quad \Lambda=-52.000 \quad \operatorname{SUSY}=(0,0) \quad \operatorname{dim}(H)=15$} \\
\hline$\Psi_{128}$ & 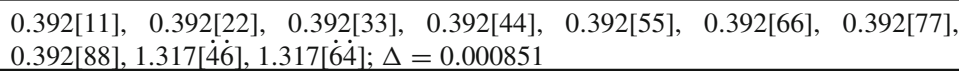 \\
\hline $\mathcal{M}_{s} / g^{2}$ & $\begin{array}{l}-73.986,-60.000(\times 20),-36.000(\times 15),-33.000(\times 12),-13.986,-9.000(\times 20), \\
0.000(\times 41), 48.000(\times 15), 217.986,252.000,277.986\end{array}$ \\
\hline $\mathcal{M}_{v} / g$ & $-12.000,-9.950(\times 12), 0.000(\times 102), 9.950(\times 12), 12.000$ \\
\hline$A_{1}$ & $-6.614(\times 8), 6.614(\times 8)$ \\
\hline$A_{3}$ & $\begin{array}{l}-19.843(\times 8),-13.747(\times 8),-3.969(\times 40),-2.128(\times 8), 2.128(\times 8), 3.969(\times 40), \\
13.747(\times 8), 19.843(\times 8)\end{array}$ \\
\hline
\end{tabular}

\begin{tabular}{|c|c|}
\hline \multicolumn{2}{|c|}{$R=2.281 \quad \Lambda=-60.063 \quad \operatorname{SUSY}=(0,1) \quad \operatorname{dim}(H)=6$} \\
\hline$\Psi_{128}$ & $0.899[55], 0.899[78], 0.899[\dot{1} \dot{7}], 0.899[\dot{3} \dot{6}], 0.931[\dot{8} \dot{2}] ; \Delta=0.001051$ \\
\hline $\mathcal{M}_{s} / g^{2}$ & $\begin{array}{l}-60.000(\times 5),-59.500,-55.000(\times 3),-52.500(\times 24),-40.338(\times 5),-37.500(\times 8) \\
-27.000(\times 16),-25.127(\times 5), 0.000(\times 50), 70.838(\times 5), 167.627(\times 5), 273.000\end{array}$ \\
\hline $\mathcal{M}_{v} / g$ & $\begin{array}{l}-13.500(\times 3),-10.000(\times 8),-4.500(\times 4),-3.000(\times 3),-2.000(\times 3),-1.000(\times 4), \\
0.000(\times 78), 1.000(\times 7), 4.500(\times 7), 10.000(\times 5), 11.000(\times 3), 12.000(\times 3)\end{array}$ \\
\hline$A_{1}$ & $-8.375(\times 4),-5.875(\times 3),-3.875,6.125(\times 5), 8.125(\times 3)$ \\
\hline$A_{3}$ & $\begin{array}{l}-25.125(\times 4),-17.625(\times 3),-15.316(\times 5),-9.625(\times 3),-8.625(\times 8),-6.125(\times 8), \\
-3.625(\times 5),-3.125,-1.625(\times 3),-1.125(\times 24), \quad 0.566(\times 5), \quad 0.875(\times 3), \\
1.875(\times 16), 2.036(\times 5), 2.875(\times 4), 4.875(\times 7), 8.375(\times 7), 11.214(\times 5), 14.375, \\
14.875(\times 3), 18.375(\times 5), 24.375(\times 3)\end{array}$ \\
\hline
\end{tabular}

\begin{tabular}{|c|c|}
\hline \multicolumn{2}{|c|}{$R=2.339 \quad \Lambda=-66.459 \quad \operatorname{SUSY}=(0,0) \quad \operatorname{dim}(H)=9$} \\
\hline$\Psi_{128}$ & $0.136[13], 1.071[22], 0.744[71], 0.698[\dot{3} \mathrm{i}] ; \Delta=0.001254$ \\
\hline $\mathcal{M}_{s} / g^{2}$ & $\begin{array}{l}-76.717(\times 5), \quad-68.398(\times 4), \quad-53.031(\times 12), \quad-52.997(\times 8), \quad-48.185(\times 16), \\
-47.007,-41.121(\times 4),-39.320(\times 12),-13.593(\times 8), 0.000(\times 47), 50.092(\times 5), \\
83.699,167.796(\times 3), 202.491,339.258\end{array}$ \\
\hline $\mathcal{M}_{v} / g$ & $\begin{array}{l}-13.158,-13.157(\times 2),-12.026(\times 4),-8.483(\times 4),-4.346(\times 3),-2.801(\times 8) \\
0.000(\times 84), 2.801(\times 8), 4.346(\times 3), 8.483(\times 4), 12.026(\times 4), 13.157(\times 2), 13.158\end{array}$ \\
\hline$A_{1}$ & $-8.528(\times 4),-7.867,-4.642(\times 3), 4.642(\times 3), 7.867,8.528(\times 4)$ \\
\hline$A_{3}$ & $\begin{array}{l}-25.583(\times 4),-23.601,-16.538(\times 3),-14.192,-13.926(\times 3),-10.191(\times 4), \\
-7.849(\times 8),-5.734(\times 5),-5.022(\times 4),-2.457(\times 4),-2.247(\times 8),-1.064(\times 3), \\
-0.222(\times 4),-0.133(\times 12), 0.133(\times 12), 0.222(\times 4), 1.064(\times 3), 2.247(\times 8), \\
2.457(\times 4), 5.022(\times 4), 5.734(\times 5), 7.849(\times 8), 10.191(\times 4), 13.926(\times 3), 14.192, \\
16.538(\times 3), 23.601,25.583(\times 4)\end{array}$ \\
\hline
\end{tabular}




\begin{tabular}{|c|c|}
\hline \multicolumn{2}{|c|}{$R=2.370 \quad \Lambda=-65.744 \quad \operatorname{SUSY}=(0,0) \quad \operatorname{dim}(H)=1$} \\
\hline$\Psi_{128}$ & 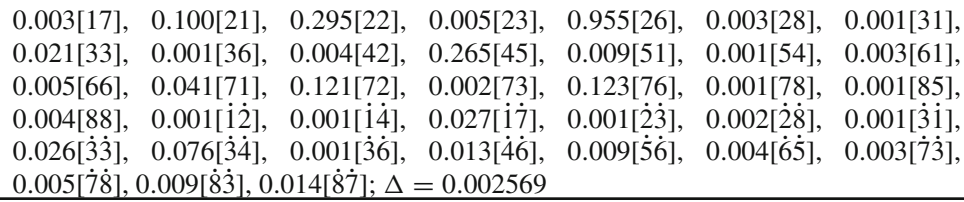 \\
\hline $\mathcal{M}_{s} / g^{2}$ & $\begin{array}{l}-78.457,-77.063,-75.306,-69.065,-65.952(\times 4),-62.258(\times 4),-60.856, \\
-59.842(\times 2),-57.429,-57.035(\times 2),-55.290,-54.648(\times 4),-53.500(\times 2), \\
-52.928(\times 2),-51.380(\times 2),-49.712(\times 2),-44.922(\times 4),-39.781(\times 2), \\
-37.424(\times 2),-36.647,-36.239(\times 4),-29.970(\times 4),-26.558,-26.364(\times 4), \\
-25.874,-25.566(\times 2),-24.244(\times 2),-24.133,-22.533,-18.808,0.000(\times 55), \\
70.708,73.569,133.551,142.236(\times 4), 151.200(\times 2), 252.334(\times 2), 293.903\end{array}$ \\
\hline $\mathcal{M}_{v} / g$ & $\begin{array}{l}-13.756,-13.532(\times 2),-12.172,-11.779(\times 2),-11.761, \quad-10.264(\times 2), \\
-9.476(\times 2),-9.466(\times 2),-5.177(\times 2),-3.655(\times 2),-3.277,-1.873,-1.840(\times 2), \\
-1.065(\times 2),-0.448(\times 2),-0.396(\times 2), 0.000(\times 74), 0.396(\times 2), 0.448(\times 2), \\
1.065(\times 2), 1.840(\times 2), 1.873,3.277,3.655(\times 2), 5.177(\times 2), 9.466(\times 2), 9.476(\times 2), \\
10.264(\times 2), 11.761,11.779(\times 2), 12.172,13.532(\times 2), 13.756\end{array}$ \\
\hline$A_{1}$ & $\begin{array}{l}-9.139(\times 2),-7.849(\times 2),-7.450,-6.646(\times 2),-5.428,5.428,6.646(\times 2), 7.450, \\
7.849(\times 2), 9.139(\times 2)\end{array}$ \\
\hline$A_{3}$ & $\begin{array}{l}-27.416(\times 2),-23.546(\times 2),-22.349,-19.937(\times 2),-16.285,-16.057(\times 2), \\
-15.987(\times 2),-15.475,-15.146,-11.985(\times 2),-10.849,-10.429(\times 2), \\
-8.238(\times 2),-8.234,-7.806(\times 2),-7.595(\times 2),-7.525(\times 2),-7.281(\times 2),-6.524, \\
-3.797(\times 2),-3.684(\times 2),-3.658(\times 2),-3.168(\times 2),-2.763,-2.697,-2.690(\times 2), \\
-2.447,-2.182(\times 2),-2.079(\times 2),-1.701(\times 2),-1.403(\times 2),-1.380,-1.338, \\
-1.240(\times 2),-1.175(\times 2),-1.037(\times 2),-0.902(\times 2),-0.880,-0.583,0.583,0.880, \\
0.902(\times 2), 1.037(\times 2), 1.175(\times 2), 1.240(\times 2), 1.338,1.380,1.403(\times 2), 1.701(\times 2), \\
2.079(\times 2), 2.182(\times 2), 2.447,2.690(\times 2), 2.697,2.763,3.168(\times 2), 3.658(\times 2), \\
3.684(\times 2), 3.797(\times 2), 6.524,7.281(\times 2), 7.525(\times 2), 7.595(\times 2), 7.806(\times 2), \\
8.234,8.238(\times 2), 10.429(\times 2), 10.849,11.985(\times 2), 15.146,15.475,15.987(\times 2), \\
16.057(\times 2), 16.285,19.937(\times 2), 22.349,23.546(\times 2), 27.416(\times 2)\end{array}$ \\
\hline
\end{tabular}

\begin{tabular}{|c|c|}
\hline \multicolumn{2}{|c|}{$R=2.411 \quad \Lambda=-71.204 \quad \operatorname{SUSY}=(1,0) \quad \operatorname{dim}(H)=5$} \\
\hline$\Psi_{128}$ & $1.009[17], 0.611[24], 1.009[81], 0.083[\dot{3} \mathrm{i}] ; \Delta=0.000955$ \\
\hline $\mathcal{M}_{s} / g^{2}$ & $\begin{array}{lllll}-71.151(\times 4), & -70.963(\times 2), & -70.395(\times 5), & -66.463(\times 4), & -61.439(\times 4), \\
-59.453(\times 3), & -58.086(\times 4), & -56.997(\times 2), & -54.963(\times 8), & -48.776(\times 2), \\
-47.198(\times 4), & -47.197(\times 4), & -37.920(\times 8), & -32.855(\times 2), & -26.802(\times 4), \\
-17.799(\times 4), & 0.000(\times 51), \quad 34.698(\times 2), & 66.419(\times 2), & 103.761(\times 3), & 114.278, \\
179.367(\times 2), 241.354(\times 2), 352.963 & \end{array}$ \\
\hline $\mathcal{M}_{v} / g$ & 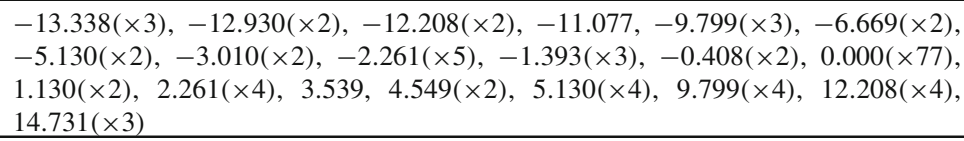 \\
\hline$A_{1}$ & $-9.119(\times 3),-7.988(\times 2),-5.580(\times 3), 4.219,5.350(\times 2), 7.758,9.350(\times 4)$ \\
\hline$A_{3}$ & $\begin{array}{l}-27.356(\times 3),-23.965(\times 2),-17.149(\times 2),-16.741(\times 3),-16.376,-15.296, \\
-13.460(\times 2),-11.610(\times 2),-10.888(\times 2),-9.400,-9.350(\times 2),-7.229(\times 2), \\
-6.480(\times 5),-6.072(\times 2),-5.612(\times 3),-4.627(\times 2),-3.089(\times 4),-1.974(\times 2), \\
-1.958(\times 4),-1.550(\times 8),-0.681(\times 8),-0.517(\times 2), 0.189(\times 8), 0.330(\times 2), \\
0.450(\times 2), 0.791(\times 3), 1.094(\times 4), 2.042(\times 4), 3.319(\times 5), 4.450(\times 4), 4.711(\times 2), \\
5.580(\times 4), \quad 7.841(\times 4), 7.988(\times 4), 10.512(\times 3), 10.883(\times 4), 15.950(\times 2), \\
16.049(\times 2), 17.447(\times 3), 23.273,28.049(\times 4)\end{array}$ \\
\hline
\end{tabular}




\begin{tabular}{|c|c|}
\hline \multicolumn{2}{|c|}{$R=2.434 \quad \Lambda=-70.269 \quad \operatorname{SUSY}=(0,0) \quad \operatorname{dim}(H)=2$} \\
\hline$\Psi_{128}$ & $0.093[38], 0.005[55], 0.093[82], 0.945[\dot{3} 7 \dot{7}], 0.005[\dot{5} \dot{6}], 0.093[\dot{7} \dot{8}] ; \Delta=0.000638$ \\
\hline $\mathcal{M}_{s} / g^{2}$ & $\begin{array}{l}-91.259,-82.458(\times 4),-65.898(\times 4),-63.223(\times 4),-62.305,-60.951(\times 4), \\
-59.727,-57.647(\times 8),-47.952(\times 4),-47.419(\times 4),-38.654(\times 4),-35.631(\times 2), \\
-34.282(\times 4),-27.232,-27.231(\times 3),-23.730(\times 4),-23.308,-23.049(\times 4), \\
-13.366,0.000(\times 54), 63.933(\times 4), 120.950(\times 4), 174.272,220.893(\times 4), 288.134, \\
312.009\end{array}$ \\
\hline $\mathcal{M}_{v} / g$ & 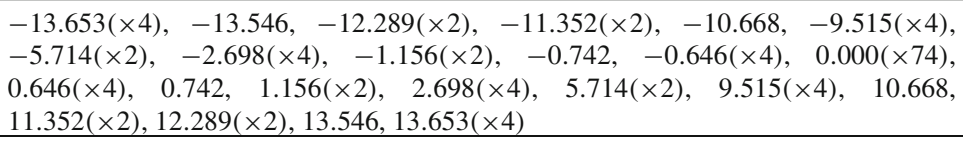 \\
\hline$A_{1}$ & $-9.941,-8.339(\times 4),-7.439,-6.650(\times 2), 6.650(\times 2), 7.439,8.339(\times 4), 9.941$ \\
\hline$A_{3}$ & $\begin{array}{l}-29.822,-25.016(\times 4),-22.318,-19.951(\times 2),-16.339(\times 4),-16.286, \\
-16.188(\times 2),-13.342(\times 2),-11.162,-9.285(\times 4),-9.139(\times 4),-8.910, \\
-7.197(\times 4),-5.449,-4.269(\times 4),-3.865(\times 4),-3.081(\times 2),-2.969,-2.855(\times 2), \\
-2.428(\times 4),-2.011(\times 2),-1.800(\times 4),-1.759,-1.688(\times 2),-1.588(\times 2), \\
-0.651(\times 4), 0.651(\times 4), 1.588(\times 2), 1.688(\times 2), 1.759,1.800(\times 4), 2.011(\times 2), \\
2.428(\times 4), 2.855(\times 2), 2.969,3.081(\times 2), 3.865(\times 4), 4.269(\times 4), 5.449,7.197(\times 4), \\
8.910,9.139(\times 4), 9.285(\times 4), 11.162,13.342(\times 2), 16.188(\times 2), 16.286,16.339(\times 4), \\
19.951(\times 2), 22.318,25.016(\times 4), 29.822\end{array}$ \\
\hline
\end{tabular}

\begin{tabular}{|c|c|}
\hline \multicolumn{2}{|c|}{$R=2.440 \quad \Lambda=-72.983 \quad \operatorname{SUSY}=(0,0) \quad \operatorname{dim}(H)=2$} \\
\hline$\Psi_{128}$ & $\begin{array}{l}0.001[26], \quad 0.615[27], \quad 0.001[32], \quad 0.001[48], \quad 0.001[81], \quad 0.309[\dot{1} \dot{8}], \quad 0.178[\dot{2} \dot{2}], \\
0.307[\dot{3} \dot{7}], 0.001[\dot{5} \dot{4}], 0.309[\dot{6} \dot{7}], 0.001[\dot{7} \dot{2}], 0.178[\dot{7} \dot{3}], 0.307[\dot{8} \dot{8}] ; \Delta=0.001933\end{array}$ \\
\hline $\mathcal{M}_{s} / g^{2}$ & $\begin{array}{l}-84.872(\times 2),-83.516,-75.878(\times 4),-72.056(\times 2),-72.055(\times 2),-69.407, \\
-69.311(\times 2),-67.582(\times 2),-64.251(\times 4),-63.978,-60.301(\times 4),-59.912, \\
-58.070(\times 2),-51.152(\times 2),-49.569(\times 2),-46.358(\times 4),-46.088(\times 2),-46.008, \\
-45.683(\times 3),-45.682,-40.375(\times 4),-36.128,-35.718(\times 2),-35.247(\times 2), \\
-29.958(\times 2),-22.583(\times 4),-16.807(\times 2), 0.000(\times 54), 27.193(\times 2), 42.845, \\
101.245,104.250,115.806(\times 2), 159.468(\times 4), 290.616(\times 2), 354.487\end{array}$ \\
\hline $\mathcal{M}_{v} / g$ & $\begin{array}{l}-14.471(\times 2), \quad-14.452,-12.992(\times 2),-12.188(\times 2), \quad-11.655, \quad-11.038, \\
-9.434(\times 2),-9.020(\times 2),-5.448(\times 2),-2.892, \quad-2.781(\times 2), \quad-2.368(\times 2), \\
-1.932(\times 2),-1.713(\times 2),-0.838,-0.235(\times 2), 0.000(\times 74), 0.235(\times 2), 0.838, \\
1.713(\times 2), 1.932(\times 2), 2.368(\times 2), 2.781(\times 2), 2.892,5.448(\times 2), 9.020(\times 2), \\
9.434(\times 2), 11.038,11.655,12.188(\times 2), 12.992(\times 2), 14.452,14.471(\times 2)\end{array}$ \\
\hline$A_{1}$ & $\begin{array}{l}-9.604(\times 2),-8.850(\times 2),-8.527,-5.997(\times 2),-5.143,5.143,5.997(\times 2), 8.527, \\
8.850(\times 2), 9.604(\times 2)\end{array}$ \\
\hline$A_{3}$ & $\begin{array}{l}-28.811(\times 2),-26.549(\times 2),-25.580,-17.992(\times 2),-17.692(\times 2),-17.018, \\
-16.274(\times 2),-15.428,-15.106,-11.988(\times 2),-11.754(\times 2),-9.243, \\
-8.921(\times 2),-8.030(\times 2),-7.903,-7.665(\times 2),-7.127(\times 2),-6.636,-6.460(\times 2), \\
-5.019(\times 2),-4.548(\times 2),-4.540(\times 2),-3.402(\times 2),-3.263(\times 2),-3.094(\times 2), \\
-2.672(\times 2),-2.493,-2.140(\times 2),-1.811(\times 2),-1.521(\times 2),-1.353(\times 2), \\
-1.217(\times 2),-1.005(\times 2),-0.746(\times 2),-0.629,-0.397(\times 2),-0.238,0.238, \\
0.397(\times 2), 0.629,0.746(\times 2), 1.005(\times 2), 1.217(\times 2), 1.353(\times 2), 1.521(\times 2), \\
1.811(\times 2), 2.140(\times 2), 2.493,2.672(\times 2), 3.094(\times 2), 3.263(\times 2), 3.402(\times 2), \\
4.540(\times 2), 4.548(\times 2), 5.019(\times 2), 6.460(\times 2), 6.636,7.127(\times 2), 7.665(\times 2), 7.903, \\
8.030(\times 2), 8.921(\times 2), 9.243,11.754(\times 2), 11.988(\times 2), 15.106,15.428,16.274(\times 2), \\
17.018,17.692(\times 2), 17.992(\times 2), 25.580,26.549(\times 2), 28.811(\times 2)\end{array}$ \\
\hline
\end{tabular}




\begin{tabular}{|c|c|}
\hline \multicolumn{2}{|c|}{$R=2.491 \quad \Lambda=-75.557 \quad \operatorname{SUSY}=(0,0) \quad \operatorname{dim}(H)=2$} \\
\hline$\Psi_{128}$ & 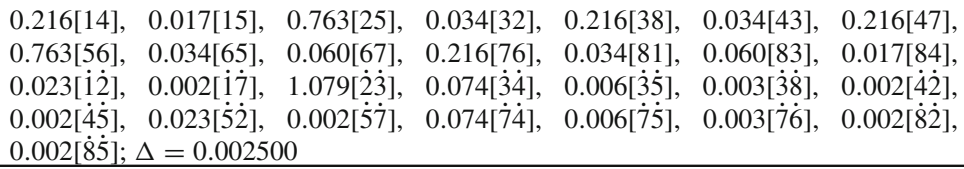 \\
\hline $\mathcal{M}_{s} / g^{2}$ & $\begin{array}{l}-90.613,-87.470(\times 2),-81.455,-77.977(\times 2),-76.164(\times 2), \quad-75.268, \\
-74.246(\times 2),-70.508(\times 2),-65.907(\times 2),-64.320(\times 6),-62.397(\times 2),-55.538, \\
-54.660(\times 4),-51.442(\times 2),-45.886(\times 2),-45.557,-45.199(\times 4),-43.665(\times 2), \\
-37.078(\times 2),-35.507(\times 2),-35.052(\times 2),-34.746,-32.420(\times 2),-31.788, \\
-31.012(\times 2),-26.032(\times 2),-22.932(\times 4),-21.068(\times 2), 0.000(\times 54), 56.667(\times 2), \\
103.172,103.173,147.343(\times 4), 147.980,225.472,228.232(\times 2), 280.984(\times 2), \\
340.472\end{array}$ \\
\hline $\mathcal{M}_{v} / g$ & $\begin{array}{l}-14.939(\times 2),-14.906,-13.535(\times 2),-12.417(\times 2),-12.147,-10.71 \\
-10.631(\times 2),-6.251(\times 2),-5.889(\times 2),-4.465,-1.941(\times 2),-1.89 \\
-1.291,-0.701(\times 2),-0.660(\times 2), 0.000(\times 74), 0.660(\times 2), 0.701(\times 2), \\
1.896(\times 2), 1.941(\times 2), 4.465,5.889(\times 2), 6.251(\times 2), 10.631(\times 2), 10.71 \\
12.147,12.417(\times 2), 13.535(\times 2), 14.906,14.939(\times 2)\end{array}$ \\
\hline$A_{1}$ & $\begin{array}{l}-10.249(\times 2),-8.427(\times 2),-8.155(\times 2),-6.333(\times 2), \quad 6.333(\times 2), 8.155(\times 2), \\
8.427(\times 2), 10.249(\times 2)\end{array}$ \\
\hline$A_{3}$ & $\begin{array}{l}-30.748(\times 2), \quad-25.280(\times 2), \quad-24.466(\times 2), \quad-18.999(\times 2), \quad-17.710(\times 2), \\
-16.993(\times 2), \quad-16.743(\times 2), \quad-14.335(\times 2), \quad-13.485(\times 2), \quad-10.717(\times 2), \\
-10.265(\times 2),-9.480(\times 2),-8.049(\times 2),-7.827(\times 2),-7.406(\times 2),-6.557(\times 2), \\
-6.427(\times 2),-5.379(\times 2),-4.361(\times 2),-3.321(\times 2),-3.235(\times 2),-3.192(\times 2), \\
-2.853(\times 2),-2.336(\times 2),-1.823(\times 2),-1.706(\times 2),-1.244(\times 2),-1.231(\times 2), \\
-1.021(\times 2),-0.995(\times 2),-0.488(\times 2),-0.286(\times 2), 0.286(\times 2), 0.488(\times 2), \\
0.995(\times 2), 1.021(\times 2), 1.231(\times 2), 1.244(\times 2), 1.706(\times 2), 1.823(\times 2), 2.336(\times 2), \\
2.853(\times 2), 3.192(\times 2), 3.235(\times 2), 3.321(\times 2), 4.361(\times 2), 5.379(\times 2), 6.427(\times 2), \\
6.557(\times 2), 7.406(\times 2), 7.827(\times 2), 8.049(\times 2), 9.480(\times 2), 10.265(\times 2), 10.717(\times 2), \\
13.485(\times 2), \quad 14.335(\times 2), 16.743(\times 2), 16.993(\times 2), 17.710(\times 2), 18.999(\times 2), \\
24.466(\times 2), 25.280(\times 2), 30.748(\times 2)\end{array}$ \\
\hline
\end{tabular}

\begin{tabular}{|c|c|}
\hline \multicolumn{2}{|c|}{$R=2.515 \quad \Lambda=-77.399 \quad \operatorname{SUSY}=(0,0) \quad \operatorname{dim}(H)=0$} \\
\hline$\Psi_{128}$ & 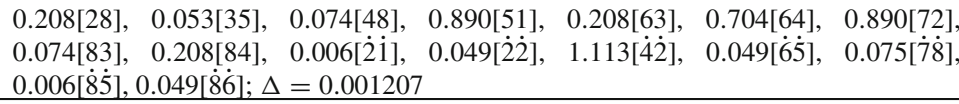 \\
\hline $\mathcal{M}_{s} / g^{2}$ & $\begin{array}{l}-94.774,-89.755,-89.754,-88.089,-87.621,-87.465,-77.255,-76.974(\times 2), \\
-76.209(\times 2),-73.198,-71.585,-70.913,-70.552(\times 2),-67.038,-64.048, \\
-62.000,-61.905,-60.495(\times 2),-58.376,-58.344(\times 2),-56.402(\times 2),-54.745, \\
-51.266,-51.014,-49.118,-48.984(\times 2),-47.771,-43.489,-43.222,-42.864, \\
-41.170,-40.555(\times 2),-36.529,-36.528,-34.844(\times 2),-30.154(\times 2),-29.770, \\
-29.045,-28.964,-28.216,-27.953,-26.301,-23.071(\times 2),-19.752,-18.763, \\
0.000(\times 56), 42.474,58.267,105.663(\times 2), 110.680,132.824,137.345,145.530(\times 2), \\
198.922,204.536,245.812,245.813,283.108,324.781,346.046\end{array}$ \\
\hline $\mathcal{M}_{v} / g$ & $\begin{array}{l}-14.797,-14.794(\times 2),-14.115,-13.695(\times 2),-13.035,-11.815(\times 2),-11.626, \\
-10.791,-10.521,-9.802(\times 2),-6.155,-5.335(\times 2),-5.147,-2.014,-1.902, \\
-1.897(\times 2),-1.020(\times 2),-0.971,-0.768,-0.246(\times 2), 0.000(\times 72), 0.246(\times 2), \\
0.768,0.971,1.020(\times 2), 1.897(\times 2), 1.902,2.014,5.147,5.335(\times 2), 6.155, \\
9.802(\times 2), 10.521,10.791,11.626,11.815(\times 2), 13.035,13.695(\times 2), 14.115, \\
14.794(\times 2), 14.797\end{array}$ \\
\hline$A_{1}$ & $\begin{array}{l}-10.628,-9.433(\times 2),-8.657,-8.448,-7.494(\times 2),-6.477,6.477,7.494(\times 2), \\
8.448,8.657,9.433(\times 2), 10.628\end{array}$ \\
\hline
\end{tabular}




\begin{tabular}{|c|c|}
\hline$A_{3}$ & $\begin{array}{l}-31.883,-28.298(\times 2),-25.970,-25.345,-22.481(\times 2),-19.432,-17.655, \\
-17.619,-17.358(\times 2),-16.963,-16.847(\times 2),-14.616,-13.949,-13.003(\times 2), \\
-10.996,-10.766,-9.926(\times 2),-9.642,-8.751,-8.267,-8.036(\times 2),-7.431, \\
-6.956,-6.615(\times 2),-6.461(\times 2),-6.364,-6.269,-4.759,-4.283,-4.236, \\
-3.836(\times 2),-3.529,-3.335(\times 2),-3.133,-2.801(\times 2),-2.507,-2.156(\times 2), \\
-2.039,-1.926,-1.861,-1.790(\times 2),-1.443,-1.406,-1.328,-1.284(\times 2), \\
-0.972,-0.612(\times 2),-0.158,0.158,0.612(\times 2), 0.972,1.284(\times 2), 1.328,1.406, \\
1.443,1.790(\times 2), 1.861,1.926,2.039,2.156(\times 2), 2.507,2.801(\times 2), 3.133, \\
3.335(\times 2), 3.529,3.836(\times 2), 4.236,4.283,4.759,6.269,6.364,6.461(\times 2), \\
6.615(\times 2), 6.956,7.431,8.036(\times 2), 8.267,8.751,9.642,9.926(\times 2), 10.766,10.996, \\
13.003(\times 2), 13.949,14.616,16.847(\times 2), 16.963,17.358(\times 2), 17.619,17.655, \\
19.432,22.481(\times 2), 25.345,25.970,28.298(\times 2), 31.883\end{array}$ \\
\hline
\end{tabular}

\begin{tabular}{|c|c|}
\hline \multicolumn{2}{|c|}{$R=2.534 \quad \Lambda=-78.875 \quad \operatorname{SUSY}=(0,0) \quad \operatorname{dim}(H)=2$} \\
\hline$\Psi_{128}$ & $\begin{array}{l}0.825[12],-0.069[26], 0.069[37],-0.069[43], 0.825[58],-0.825[64],-0.825[71], \\
0.069[85],-0.005[\dot{1} \dot{6}],-1.153[\dot{2} \dot{2}], 0.005[\dot{3} \dot{3}], 1.008[\dot{4} \dot{7}],-0.082[\dot{5} \dot{4}], 0.005[\dot{6} \dot{8}], \\
1.153[\dot{7} \dot{5}], 0.005[\dot{8} \dot{1}] ; \Delta=0.000714\end{array}$ \\
\hline $\mathcal{M}_{s} / g^{2}$ & $\begin{array}{l}-101.966,-95.668(\times 4),-85.332,-83.178(\times 4),-77.936(\times 2),-71.825(\times 4), \\
-70.814,-61.307(\times 4),-57.793,-56.584,-54.488,-53.356(\times 4),-49.458(\times 4), \\
-46.077(\times 4),-45.509(\times 2),-40.337(\times 4),-33.701(\times 2),-30.127(\times 4),-27.039, \\
-23.613,-19.692(\times 4),-18.615,0.000(\times 54), 40.014(\times 4), 86.707(\times 4), 137.635, \\
176.645,177.956(\times 4), 223.790(\times 2), 338.103,349.795,354.699\end{array}$ \\
\hline $\mathcal{M}_{v} / g$ & $\begin{array}{l}-14.679,-14.511(\times 2),-14.034(\times 4),-11.648(\times 4),-11.492(\times 2),-10.303, \\
-5.446(\times 4),-1.922(\times 4),-1.060(\times 4), \quad 0.000(\times 76), \quad 1.060(\times 4), 1.922(\times 4), \\
5.446(\times 4), 10.303,11.492(\times 2), 11.648(\times 4), 14.034(\times 4), 14.511(\times 2), 14.679\end{array}$ \\
\hline$A_{1}$ & $-9.765(\times 4),-7.596(\times 4), 7.596(\times 4), 9.765(\times 4)$ \\
\hline$A_{3}$ & $\begin{array}{l}-29.296(\times 4), \quad-22.787(\times 4),-17.359(\times 4), \quad-17.249(\times 4), \quad-13.491(\times 4), \\
-9.631(\times 4),-7.570(\times 4),-7.360(\times 8),-4.514(\times 4),-3.737(\times 4),-2.686(\times 8), \\
-2.424(\times 4),-1.781(\times 4),-0.864(\times 4), \quad 0.864(\times 4), \quad 1.781(\times 4), 2.424(\times 4), \\
2.686(\times 8), 3.737(\times 4), 4.514(\times 4), 7.360(\times 8), 7.570(\times 4), 9.631(\times 4), 13.491(\times 4), \\
17.249(\times 4), 17.359(\times 4), 22.787(\times 4), 29.296(\times 4)\end{array}$ \\
\hline
\end{tabular}

\begin{tabular}{|c|c|}
\hline \multicolumn{2}{|c|}{$R=2.546 \quad \Lambda=-81.651 \quad \operatorname{SUSY}=(0,0) \quad \operatorname{dim}(H)=1$} \\
\hline$\Psi_{128}$ & 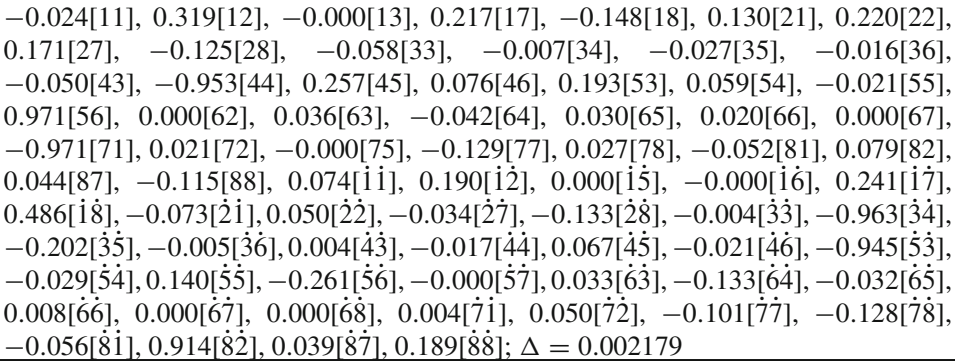 \\
\hline
\end{tabular}




\begin{tabular}{|c|c|}
\hline $\mathcal{M}_{s} / g^{2}$ & $\begin{array}{l}-87.572,-84.933,-84.763(\times 2),-83.630(\times 2),-83.585,-83.287(\times 2), \\
-81.743(\times 2),-75.519,-74.380(\times 2),-73.128(\times 2),-70.402(\times 2),-68.972(\times 2), \\
-68.612,-68.421,-65.433(\times 2),-62.128(\times 2),-61.929(\times 2),-57.874(\times 2), \\
-57.395(\times 2),-52.025(\times 2),-51.222,-48.388,-48.101,-45.155(\times 2), \\
-44.462(\times 2),-41.581(\times 2),-41.183(\times 2),-39.672,-37.429(\times 2),-35.727(\times 2), \\
-32.620(\times 2),-30.629,-20.424(\times 2),-15.832(\times 2), 0.000(\times 55), 32.548, \\
91.218(\times 2), 99.393,99.394,116.438,144.278(\times 2), 151.969,156.115,252.501, \\
258.051(\times 2), 303.566(\times 2), 384.245\end{array}$ \\
\hline $\mathcal{M}_{v} / g$ & $\begin{array}{l}-16.035(\times 2),-15.750,-14.080(\times 2),-12.437(\times 2),-12.407(\times 2),-12.166, \\
-10.500,-10.359(\times 2),-6.801(\times 2),-6.011(\times 2),-3.094,-2.698(\times 2),-2.515, \\
-1.720(\times 2),-1.671(\times 2),-0.475(\times 2), 0.000(\times 73), 0.451(\times 2), 0.567,1.467, \\
1.489,1.499(\times 2), 2.698,3.297(\times 2), 4.046(\times 2), 5.575,7.308(\times 2), 10.174,10.359, \\
10.500(\times 2), 12.437,13.376(\times 2), 13.839,14.037(\times 2), 15.188(\times 2), 15.409\end{array}$ \\
\hline$A_{1}$ & $\begin{array}{l}-10.770(\times 2),-9.387(\times 2),-9.153,-7.262,-6.036(\times 2), 5.841,7.462(\times 2), 7.970, \\
9.345(\times 2), 10.569,10.807\end{array}$ \\
\hline$A_{3}$ & $\begin{array}{l}-32.310(\times 2),-28.160(\times 2),-27.460,-21.785,-18.994(\times 2),-18.109(\times 2), \\
-17.846,-17.426(\times 2),-17.289,-15.301(\times 2),-13.813,-11.909,-11.376(\times 2), \\
-10.868,-10.516(\times 2),-9.933(\times 2),-8.472,-8.471,-7.141(\times 2),-6.924, \\
-6.514(\times 2),-6.426(\times 2),-5.901(\times 2),-4.963,-4.279(\times 2),-3.535(\times 2),-3.388, \\
-3.320(\times 2),-3.302,-2.904(\times 2),-2.873,-2.532,-2.269(\times 2),-1.833(\times 2), \\
-1.761(\times 2),-1.109(\times 2),-0.950(\times 2),-0.692(\times 2),-0.037,-0.015,0.020(\times 2), \\
0.350(\times 2), 0.770(\times 2), 1.020(\times 2), 1.395,1.458(\times 2), 1.656(\times 2), 1.967(\times 2), \\
1.974(\times 2), 3.172(\times 2), 3.270(\times 2), 3.354,3.382,3.703(\times 2), 4.059,4.101(\times 2), \\
4.530,4.839,5.567,6.332(\times 2), 6.514(\times 2), 6.740,7.017(\times 2), 7.173,10.161(\times 2), \\
10.779,10.860(\times 2), 11.402(\times 2), 11.856(\times 2), 12.203,15.309(\times 2), 16.729,17.524, \\
17.931(\times 2), 17.978(\times 2), 18.665,22.385(\times 2), 23.911,28.034(\times 2), 31.708,32.420\end{array}$ \\
\hline
\end{tabular}

\begin{tabular}{|c|c|}
\hline \multicolumn{2}{|c|}{$R=2.554 \quad \Lambda=-82.168 \quad \operatorname{SUSY}=(0,0) \quad \operatorname{dim}(H)=0$} \\
\hline$\Psi_{128}$ & 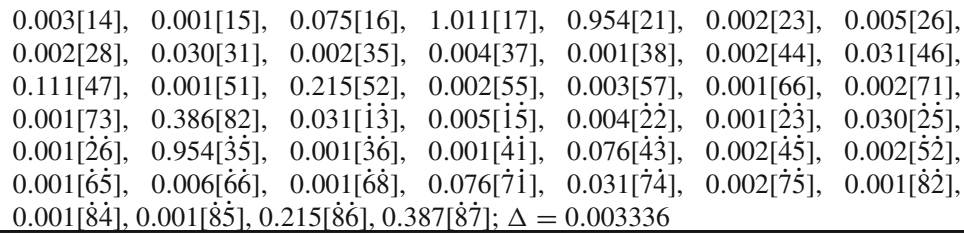 \\
\hline $\mathcal{M}_{s} / g^{2}$ & $\begin{array}{l}-92.643,-89.925,-87.938(\times 2),-87.535,-86.629(\times 2),-82.550,-80.198, \\
-80.165,-79.645,-78.365,-73.668(\times 2),-71.430(\times 2),-71.406,-71.268, \\
-71.038(\times 2),-69.154,-68.822(\times 2),-67.673,-67.101,-59.890,-58.426(\times 2), \\
-57.479,-56.823,-55.054(\times 2),-52.666,-51.273,-50.177,-48.906(\times 2), \\
-46.893,-45.254,-45.219,-43.000,-41.287(\times 2),-38.060,-37.348(\times 2), \\
-34.427,-33.859(\times 2),-33.777,-29.016,-26.871,-26.870,-26.869,-16.736, \\
0.000(\times 56), 21.567,29.888,84.557,100.326,114.263,119.334(\times 2), 125.095, \\
140.703,140.704,185.336,246.054,275.277,275.278,280.739,330.856,384.086\end{array}$ \\
\hline $\mathcal{M}_{v} / g$ & $\begin{array}{l}-15.643,-15.578,-15.516,-14.428,-13.881,-13.720,-13.370,-12.899, \\
-12.381,-11.512,-10.950,-10.394,-9.611,-9.015,-6.823,-6.074,-4.656, \\
-3.565,-3.258,-2.625,-2.392,-1.824,-1.612,-1.530,-1.462,-0.574, \\
-0.543,-0.194,0.000(\times 72), 0.194,0.543,0.574,1.462,1.530,1.612,1.824,2.392, \\
2.625,3.258,3.565,4.655,6.074,6.823,9.015,9.611,10.394,10.950,11.512,12.381, \\
12.899,13.370,13.720,13.881,14.428,15.516,15.578,15.643\end{array}$ \\
\hline$A_{1}$ & $\begin{array}{l}-10.969,-10.125,-10.008,-9.343,-8.588,-7.628,-6.869,-6.001,6.001, \\
6.869,7.628,8.588,9.343,10.008,10.125,10.969\end{array}$ \\
\hline
\end{tabular}




\begin{tabular}{|c|c|}
\hline$A_{3}$ & $\begin{array}{l}-32.908,-30.376,-30.025,-28.029,-25.763,-22.883,-20.606,-19.002, \\
-18.431,-18.003,-17.913,-17.791,-17.649,-17.100,-16.545,-15.351, \\
-13.603,-12.454,-11.159,-11.026,-10.940,-10.891,-10.852,-10.625, \\
-10.466,-10.346,-8.259,-7.151,-6.939,-6.884,-6.553,-6.276,-6.209, \\
-6.201,-6.094,-5.731,-4.669,-4.159,-4.077,-4.011,-3.932,-3.828, \\
-3.671,-3.461,-3.347,-3.234,-3.213,-3.106,-2.980,-2.417,-2.285, \\
-1.938,-1.835,-1.819,-1.708,-1.405,-1.270,-1.257,-1.220,-1.121, \\
-0.963,-0.127,-0.022,-0.018,0.018,0.022,0.127,0.963,1.121,1.220,1.257, \\
1.270,1.405,1.708,1.819,1.835,1.938,2.285,2.417,2.980,3.106,3.213,3.234, \\
3.347,3.461,3.671,3.828,3.932,4.011,4.077,4.159,4.669,5.731,6.094,6.201, \\
6.209,6.276,6.553,6.884,6.939,7.151,8.259,10.346,10.467,10.625,10.852, \\
10.891,10.940,11.026,11.159,12.454,13.603,15.351,16.545,17.100,17.649, \\
17.791,17.913,18.003,18.431,19.002,20.606,22.883,25.763,28.029,30.025, \\
30.376,32.908\end{array}$ \\
\hline
\end{tabular}

\begin{tabular}{|c|c|}
\hline \multicolumn{2}{|c|}{$R=2.563 \quad \Lambda=-81.767 \quad \operatorname{SUSY}=(0,0) \quad \operatorname{dim}(H)=1$} \\
\hline$\Psi_{128}$ & 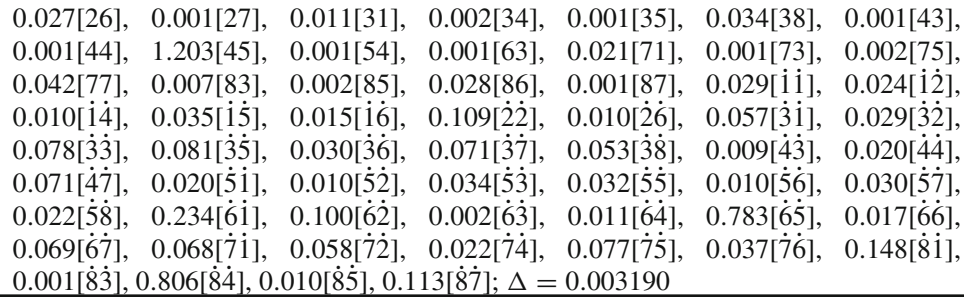 \\
\hline $\mathcal{M}_{s} / g^{2}$ & $\begin{array}{l}-104.875(\times 2),-95.578,-87.794,-87.614(\times 2),-87.391(\times 2),-83.699(\times 2), \\
-79.250(\times 2),-75.646(\times 2),-74.811,-63.478(\times 2),-63.412(\times 2),-62.808, \\
-61.508(\times 2),-60.435(\times 2),-59.076(\times 2),-55.248(\times 2),-54.959,-54.305(\times 2), \\
-51.195(\times 2),-43.229(\times 2),-41.561(\times 2),-41.067,-35.491,-35.020(\times 2), \\
-33.597(\times 2),-32.956,-30.141(\times 2),-27.359(\times 2),-27.193,-25.884, \\
-23.069(\times 2),-20.534(\times 2),-18.786,0.000(\times 55), 35.955(\times 2), 40.072,134.352, \\
137.164(\times 2), 139.194(\times 2), 171.319,224.537,224.538,230.513(\times 2), 246.686, \\
326.950(\times 2), 352.233,366.047\end{array}$ \\
\hline $\mathcal{M}_{v} / g$ & $\begin{array}{l}-18.489,-16.311(\times 2),-13.500(\times 2),-13.475,-12.652,-12.157(\times 2), \\
-11.472(\times 2),-11.181,-11.062,-10.695(\times 2),-7.050,-4.345(\times 2),-1.999, \\
-1.967(\times 2),-1.871,-1.544(\times 2),-0.764,-0.352,-0.292(\times 2), 0.000(\times 73), \\
0.325(\times 2), 0.533,1.679(\times 2), 1.999(\times 2), 2.026(\times 2), 3.835(\times 2), 9.864,10.340(\times 2), \\
11.062(\times 2), 11.472,12.652(\times 2), 13.025(\times 2), 13.449,14.583(\times 2), 14.998(\times 2), \\
15.094\end{array}$ \\
\hline$A_{1}$ & 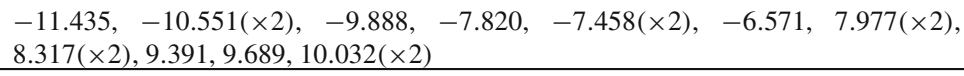 \\
\hline$A_{3}$ & $\begin{array}{l}-34.306,-31.653(\times 2),-29.663,-23.461,-22.374(\times 2),-19.721,-19.714, \\
-19.433(\times 2),-18.810,-17.582(\times 2),-17.546(\times 2),-14.749,-13.033(\times 2), \\
-12.025,-10.730(\times 2),-10.629(\times 2),-9.358,-9.059(\times 2),-8.352,-8.304(\times 2), \\
-7.936(\times 2),-7.791,-7.581(\times 2),-7.417,-5.066,-4.671(\times 2),-3.765(\times 2), \\
-3.737,-3.485(\times 2),-2.642(\times 2),-2.355(\times 2),-2.276(\times 2),-2.274(\times 2), \\
-1.996(\times 2),-1.786(\times 3),-1.571,-1.281(\times 2),-1.277(\times 2),-0.837(\times 2), \\
0.469(\times 2), 0.678(\times 2), 1.111,1.522(\times 2), 1.646(\times 2), 1.983(\times 2), 2.382,2.585(\times 2), \\
2.805,3.034,3.050(\times 2), 3.393(\times 2), 3.765(\times 2), 3.939(\times 2), 4.270,4.535(\times 2), \\
4.882(\times 2), 5.792(\times 2), 6.081,7.347,7.632(\times 2), 8.932(\times 2), 9.979(\times 2), 10.138, \\
10.555(\times 2), 11.020(\times 2), 14.916(\times 2), 15.710,15.772(\times 2), 17.295,17.422, \\
17.582(\times 2), 18.100(\times 2), 18.288,23.932(\times 2), 24.952(\times 2), 28.172,29.068, \\
30.095(\times 2)\end{array}$ \\
\hline
\end{tabular}




\begin{tabular}{|c|c|}
\hline \multicolumn{2}{|c|}{$R=2.591 \quad \Lambda=-85.757 \quad \operatorname{SUSY}=(0,0) \quad \operatorname{dim}(H)=1$} \\
\hline$\Psi_{128}$ & 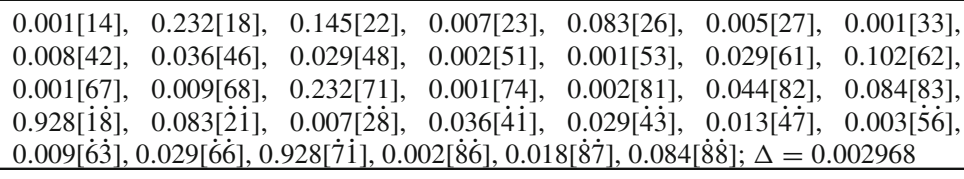 \\
\hline $\mathcal{M}_{s} / g^{2}$ & $\begin{array}{l}-110.537(\times 2),-85.154,-81.972(\times 2),-81.125(\times 2),-81.012(\times 2),-80.548, \\
-79.019(\times 2),-78.380(\times 2),-76.828(\times 2),-72.632,-72.563,-71.140, \\
-71.093,-71.092,-69.317(\times 2),-67.728(\times 2),-66.981(\times 2),-66.871, \\
-66.515(\times 2),-56.396(\times 2),-54.408(\times 2),-51.967(\times 2),-51.263(\times 2), \\
-42.318(\times 2),-36.981(\times 2),-35.868,-34.710(\times 2),-34.591(\times 2),-30.358, \\
-25.177,-24.350(\times 2),-23.754,-16.549(\times 2), 0.000(\times 55), 7.125(\times 2), 19.115, \\
27.604,128.434,144.288(\times 2), 166.525(\times 2), 197.294,219.723,219.728,254.717, \\
258.804(\times 2), 340.023,340.024,354.055,400.532\end{array}$ \\
\hline $\mathcal{M}_{v} / g$ & $\begin{array}{l}-15.291(\times 2),-14.912,-14.370(\times 2),-14.271,-13.971(\times 2),-12.710(\times 2), \\
-12.272(\times 2),-10.954,-9.999(\times 2),-8.316,-4.415(\times 2),-2.582(\times 2), \\
-1.803(\times 2),-1.374(\times 2),-1.284,-1.236(\times 2), 0.000(\times 73), 0.141,0.144(\times 2), \\
1.749,2.253,2.275(\times 2), 2.337(\times 2), 2.582,3.940(\times 2), 6.082,10.945(\times 2), \\
10.954(\times 2), 11.625,12.272,12.537(\times 2), 12.710,13.102(\times 2), 13.591,17.179(\times 2), \\
19.954\end{array}$ \\
\hline$A_{1}$ & $\begin{array}{l}-10.295(\times 2),-10.171,-9.063,-8.978(\times 2),-7.517(\times 2), 6.368,6.522(\times 2), 8.162, \\
10.987,11.290(\times 2), 11.674\end{array}$ \\
\hline$A_{3}$ & $\begin{array}{l}-30.886(\times 2),-30.512,-27.189,-26.935(\times 2),-22.551(\times 2),-18.596, \\
-18.442,-18.394(\times 2),-18.236(\times 2),-17.263,-16.300(\times 2),-13.752,-13.228, \\
-12.939(\times 2),-12.903(\times 2),-11.751(\times 2),-10.541(\times 2),-9.563(\times 2),-6.799, \\
-6.798,-5.581,-5.339(\times 2),-5.066,-4.879,-4.407(\times 2),-4.083,-4.075(\times 2), \\
-3.798(\times 2),-3.382(\times 2),-3.185,-3.184,-3.164,-3.078(\times 2),-2.992(\times 2), \\
-2.889(\times 2),-2.800,-1.923(\times 2),-1.246(\times 2),-0.922(\times 2), 0.138(\times 2), 0.518, \\
0.654(\times 2), 0.808,1.293,1.319(\times 2), 1.638(\times 2), 1.770(\times 2), 1.781(\times 2), 2.143(\times 2), \\
2.208(\times 2), 2.427(\times 2), 2.755,3.000(\times 2), 3.072(\times 2), 3.437,4.094(\times 2), 5.027, \\
5.390,7.385,7.575(\times 2), 7.806,7.918(\times 2), 8.009(\times 2), 8.776,9.380,10.204(\times 2), \\
10.815(\times 2), 11.144(\times 2), 12.084,12.156(\times 2), 13.842,17.503(\times 2), 19.091(\times 2), \\
19.103,19.566(\times 2), 19.717(\times 2), 19.851,21.076,24.486,32.961,33.871(\times 2), \\
35.021\end{array}$ \\
\hline
\end{tabular}

\begin{tabular}{|lll|}
\hline$R=2.648 \Lambda=-93.255 \quad \operatorname{SUSY}=(1,1) \operatorname{dim}(H)=2$ \\
\hline$\Psi_{128}$ & $0.875[51], \quad 0.116[67], \quad 0.875[76], \quad 0.965[84], 0.875[\dot{2} \dot{3}], \quad 0.965[3 \dot{3}], \quad 0.205[\dot{5} \dot{4}]$, \\
& $0.116[\dot{8} 1] ; \Delta=0.001631$
\end{tabular}




\begin{tabular}{|c|c|}
\hline \multicolumn{2}{|c|}{$R=2.649 \quad \Lambda=-90.252 \quad \operatorname{SUSY}=(0,0) \quad \operatorname{dim}(H)=0$} \\
\hline$\Psi_{128}$ & 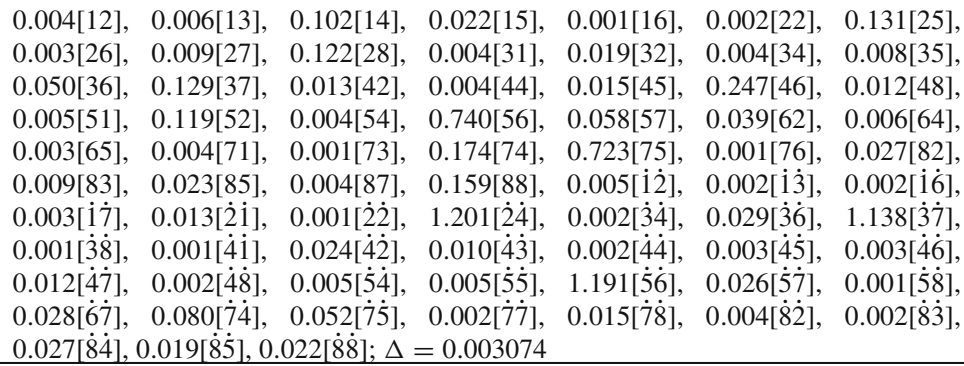 \\
\hline $\mathcal{M}_{s} / g^{2}$ & $\begin{array}{l}-107.018,-106.214,-103.360(\times 2),-101.632,-100.672,-100.257(\times 2), \\
-99.408,-95.471,-94.003(\times 2),-93.167,-89.294,-85.756,-79.281(\times 2), \\
-70.929,-65.309,-63.784(\times 2),-63.326(\times 2),-62.777,-62.058,-60.036, \\
-58.769,-55.751(\times 2),-52.450,-52.361(\times 2),-50.968,-48.652,-43.276(\times 2), \\
-41.454,-38.968,-35.746,-34.377(\times 2),-32.873,-32.318,-28.403(\times 2), \\
-23.447,-23.369(\times 2),-20.179,-20.131,-18.407,0.000(\times 56), 23.684,50.642, \\
88.806,96.319(\times 2), 100.352,110.472,144.722(\times 2), 166.977,188.870,202.588, \\
202.589,203.795,223.040,336.224,343.949(\times 2), 395.292,401.580,412.089\end{array}$ \\
\hline $\mathcal{M}_{v} / g$ & $\begin{array}{l}-17.695,-16.667,-16.535,-15.169,-15.116,-14.748,-14.563,-13.926, \\
-13.382,-12.988,-12.090,-12.004,-11.827,-11.086,-10.955,-10.385, \\
-6.417,-6.091,-1.754,-1.665(\times 2),-1.662,-1.614,-1.596,-1.412,-0.680, \\
-0.624,-0.146,0.000(\times 72), 0.146,0.624,0.680,1.412,1.596,1.614,1.662, \\
1.665(\times 2), 1.754,6.091,6.417,10.385,10.955,11.086,11.827,12.004,12.090, \\
12.988,13.382,13.926,14.563,14.748,15.116,15.169,16.535,16.667,17.695\end{array}$ \\
\hline$A_{1}$ & $\begin{array}{l}-11.684,-11.221,-10.123,-9.669,-9.532,-9.068,-8.418,-7.515,7.515, \\
8.418,9.068,9.532,9.669,10.123,11.221,11.684\end{array}$ \\
\hline$A_{3}$ & $\begin{array}{l}-35.051,-33.663,-30.369,-29.006,-28.596,-27.203,-25.254,-22.546, \\
-20.281,-20.047,-19.503,-19.397,-18.798,-18.601,-18.329,-18.307, \\
-17.747,-15.991,-15.262,-14.944,-14.033,-13.548,-12.219,-12.165, \\
-9.228,-8.933,-8.743,-8.326,-8.210,-7.988,-7.875,-7.845,-7.765, \\
-7.713,-7.531,-7.333,-7.294,-7.228,-7.053,-5.993,-5.847,-5.506, \\
-4.996,-4.698,-4.543,-4.045,-3.631,-3.115,-2.979,-2.770,-2.707, \\
-2.691,-2.300,-2.186,-2.042,-1.824,-1.570,-1.508,-1.252,-1.150, \\
-1.075,-0.745,-0.731,-0.355,0.355,0.731,0.745,1.075,1.150,1.252,1.508, \\
1.570,1.824,2.042,2.186,2.300,2.691,2.707,2.770,2.979,3.115,3.631,4.045, \\
4.543,4.698,4.996,5.506,5.847,5.993,7.053,7.228,7.294,7.333,7.531,7.713, \\
7.765,7.845,7.875,7.988,8.210,8.326,8.743,8.933,9.228,12.165,12.219,13.548, \\
14.033,14.944,15.262,15.991,17.747,18.307,18.329,18.601,18.798,19.397, \\
19.503,20.047,20.281,22.546,25.254,27.203,28.596,29.006,30.369,33.663, \\
35.051\end{array}$ \\
\hline
\end{tabular}




\begin{tabular}{|c|c|}
\hline \multicolumn{2}{|c|}{$R=2.664 \quad \Lambda=-94.136 \quad \operatorname{SUSY}=(0,0) \quad \operatorname{dim}(H)=0$} \\
\hline$\Psi_{128}$ & 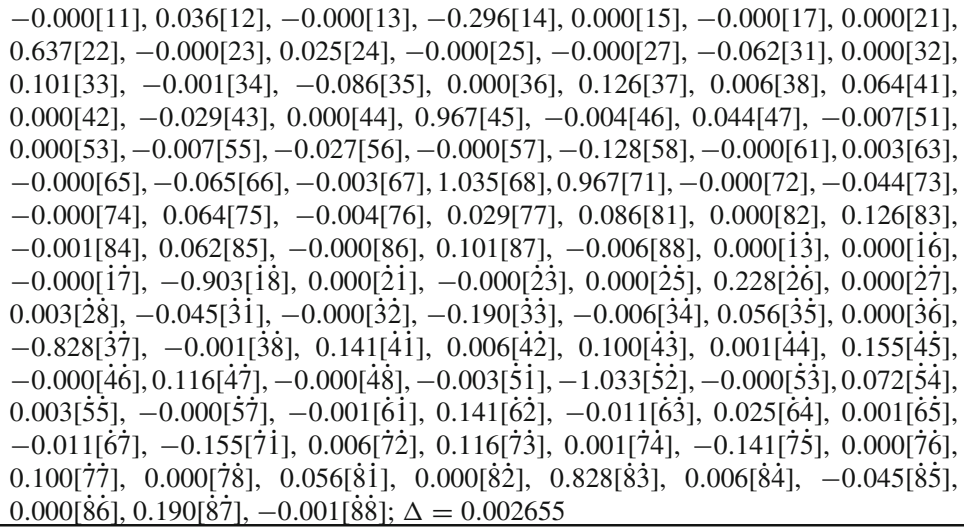 \\
\hline $\mathcal{M}_{s} / g^{2}$ & $\begin{array}{l}-100.682,-99.838,-98.191,-97.298(\times 2),-95.815,-94.189,-93.196,-93.195, \\
-93.109(\times 2),-92.411,-91.917,-88.371(\times 2),-87.426(\times 2),-85.158(\times 2), \\
-85.116,-82.078,-78.596,-75.949,-75.686,-75.685,-74.761,-72.075(\times 2), \\
-70.981,-70.561,-59.884,-57.429,-57.152,-54.811(\times 2),-51.899(\times 2), \\
-49.448,-48.479,-45.093(\times 2),-44.800,-44.580,-42.431,-42.289, \\
-42.187(\times 2),-42.092,-41.987,-36.277,-13.400,-7.991,-4.067,0.000(\times 56), \\
31.328,47.205,73.979,107.448,120.278,120.279,128.050,131.300,131.301, \\
150.555,200.656(\times 2), 205.345,205.346,244.111,280.245,432.765,435.609, \\
444.277\end{array}$ \\
\hline $\mathcal{M}_{v} / g$ & $\begin{array}{l}-16.765,-16.487,-16.050,-15.900(\times 2),-15.811(\times 2),-13.412(\times 2), \\
-12.794(\times 2),-10.828,-10.110,-8.889,-7.324,-7.111,-4.270(\times 2),-3.672, \\
-3.575(\times 2),-3.428,-1.392(\times 2),-1.194(\times 2),-0.998,-0.573,0.000(\times 72), \\
0.845,0.998,1.145(\times 2), 1.485(\times 2), 3.284(\times 2), 3.336,3.672,4.702(\times 2), 6.381, \\
7.111,8.889,10.110,11.864,12.934(\times 2), 13.822(\times 2), 15.478(\times 2), 15.497(\times 2), \\
16.050,16.487,16.493\end{array}$ \\
\hline$A_{1}$ & $\begin{array}{l}-12.139,-11.472,-11.422,-8.901(\times 2),-8.528(\times 2),-5.324,5.671,8.411(\times 2), \\
9.017(\times 2), 11.125,11.769,11.793\end{array}$ \\
\hline$A_{3}$ & $\begin{array}{l}-36.418,-34.415,-34.266,-26.702(\times 2),-25.583,-25.582,-20.279(\times 2), \\
-19.817(\times 2),-19.219,-18.878,-18.797,-15.973,-15.838,-14.740,-13.640, \\
-12.277,-12.220,-11.862,-11.741(\times 2),-11.465(\times 2),-10.301,-9.112(\times 2), \\
-8.599(\times 2),-8.596,-8.261,-6.935(\times 2),-6.194(\times 2),-5.876,-5.483,-5.035, \\
-5.015,-4.162(\times 2),-3.871,-3.844(\times 2),-3.543,-3.345(\times 2),-2.644,-2.507, \\
-2.495,-2.463,-2.134(\times 2),-2.097,-1.889,-1.694,-1.047,-0.994,-0.917, \\
-0.700(\times 2),-0.252(\times 2), 0.655(\times 2), 0.806,0.954(\times 2), 0.991,1.123,1.644,1.931, \\
2.136,2.146(\times 2), 2.360,2.583,2.619,2.671,3.284(\times 2), 3.693(\times 2), 3.705,3.803, \\
3.887(\times 2), 4.591,4.689,5.505(\times 2), 6.008,6.909,7.142(\times 2), 8.001,8.150(\times 2), \\
8.571,9.503(\times 2), 10.436,11.274,11.408(\times 2), 11.494,12.265(\times 2), 12.494, \\
13.804,15.621,16.122,17.013,18.523,18.598,18.695,19.874(\times 2), 20.113(\times 2), \\
25.232(\times 2), 27.052(\times 2), 33.375,35.307,35.378\end{array}$ \\
\hline
\end{tabular}




\begin{tabular}{|c|c|}
\hline \multicolumn{2}{|c|}{$R=2.686 \quad \Lambda=-95.538 \quad \operatorname{SUSY}=(0,0) \quad \operatorname{dim}(H)=0$} \\
\hline$\Psi_{128}$ & 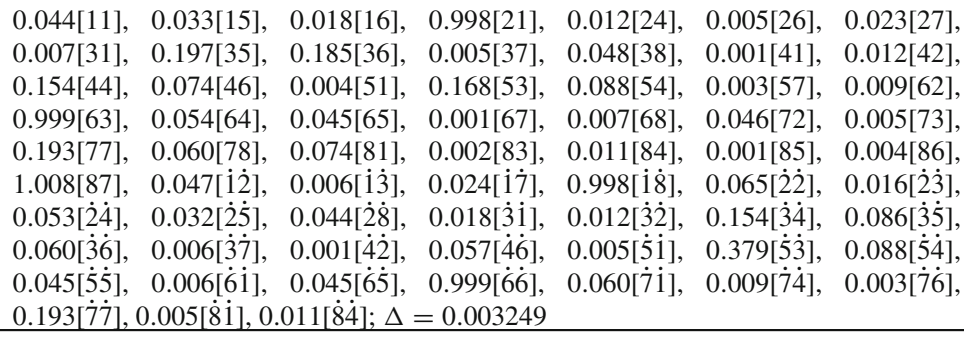 \\
\hline $\mathcal{M}_{s} / g^{2}$ & $\begin{array}{l}-116.478,-106.046,-105.552(\times 2),-96.374,-95.748,-95.747,-95.725, \\
-94.067,-92.431(\times 2),-89.622(\times 2),-87.921,-86.423,-85.537,-83.107, \\
-79.869,-79.868,-77.022,-73.502(\times 2),-73.291,-70.317(\times 2),-67.658(\times 2), \\
-65.834,-61.714(\times 2),-59.536,-58.809,-56.761,-55.436,-51.680,-51.380, \\
-50.227(\times 2),-48.048,-42.252,-39.016,-38.631,-38.630,-36.971,-33.933, \\
-33.581,-31.176,-29.518(\times 2),-18.248,-14.492,0.000(\times 56), 30.962,36.271, \\
41.820,42.024,109.880,120.214,131.297(\times 2), 154.536,154.537,172.174,205.473, \\
243.244,252.244,252.245,276.818(\times 2), 328.580,438.473,444.132,459.390\end{array}$ \\
\hline $\mathcal{M}_{v} / g$ & $\begin{array}{l}-17.270,-16.930,-16.564,-16.054,-15.841,-15.694,-15.647,-14.445, \\
-14.133,-13.376,-12.741,-12.668,-11.671,-10.464,-9.243,-7.396,-6.793, \\
-5.356,-3.155,-3.053,-3.042,-2.349,-1.783,-1.559,-1.279,-1.067, \\
-0.998,-0.933,0.000(\times 72), 0.933,0.998,1.067,1.279,1.559,1.783,2.349,3.042, \\
3.053,3.155,5.356,6.793,7.396,9.243,10.464,11.671,12.668,12.741,13.376, \\
14.133,14.445,15.647,15.694,15.841,16.054,16.564,16.930,17.270\end{array}$ \\
\hline$A_{1}$ & $\begin{array}{l}-11.823,-11.804,-11.060,-10.065,-9.428,-9.154,-8.517,-6.463,6.463, \\
8.517,9.154,9.428,10.065,11.060,11.804,11.823\end{array}$ \\
\hline$A_{3}$ & $\begin{array}{l}-35.470,-35.413,-33.181,-30.196,-28.285,-27.463,-25.552,-20.865, \\
-20.817,-20.203,-19.973,-19.841,-19.498,-19.389,-18.977,-17.574, \\
-16.686,-14.539,-14.420,-13.898,-13.127,-12.732,-12.402,-12.068, \\
-11.952,-11.228,-11.201,-9.520,-9.505,-9.142,-8.757,-8.516,-8.006, \\
-7.842,-6.888,-6.545,-6.543,-6.350,-5.580,-5.186,-4.937,-4.595, \\
-4.292,-3.854,-3.680,-3.433,-3.318,-3.191,-3.092,-2.834,-2.754, \\
-2.589,-2.092,-2.071,-1.788,-1.623,-1.617,-1.529,-1.141,-1.111, \\
-0.683,-0.513,-0.210,-0.151,0.151,0.210,0.513,0.683,1.111,1.141,1.529, \\
1.617,1.623,1.788,2.071,2.092,2.589,2.754,2.834,3.092,3.191,3.318,3.433, \\
3.680,3.854,4.292,4.595,4.937,5.186,5.580,6.350,6.543,6.545,6.888,7.842, \\
8.006,8.516,8.757,9.142,9.505,9.520,11.201,11.228,11.952,12.068,12.402, \\
12.732,13.127,13.898,14.420,14.539,16.686,17.574,18.977,19.389,19.498, \\
19.841,19.973,20.203,20.817,20.865,25.552,27.463,28.285,30.196,33.181, \\
35.413,35.470\end{array}$ \\
\hline
\end{tabular}

\begin{tabular}{|c|c|}
\hline \multicolumn{2}{|c|}{$R=2.691 \quad \Lambda=-94.399 \quad \operatorname{SUSY}=(0,0) \quad \operatorname{dim}(H)=0$} \\
\hline$\Psi_{128}$ & 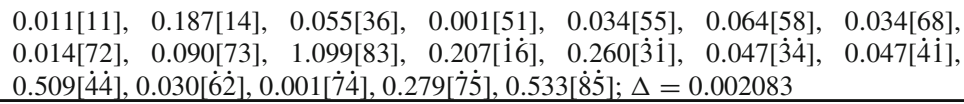 \\
\hline $\mathcal{M}_{s} / g^{2}$ & $\begin{array}{l}-121.785,-98.755(\times 2),-97.579,-93.792,-91.882(\times 2),-89.540,-88.136, \\
-83.853(\times 2),-83.774,-82.712,-80.961,-79.804(\times 2),-77.442,-75.492(\times 2), \\
-70.424,-64.680(\times 2),-64.035,-62.120,-61.485,-61.101(\times 2),-59.834(\times 2), \\
-59.595,-58.384,-55.015,-54.961,-52.921,-48.437(\times 2),-44.195,-41.897, \\
-36.390(\times 2),-31.399,-28.347(\times 2),-27.610,-27.567,-25.228,-24.399, \\
-23.262(\times 2),-19.335,-13.100,0.000(\times 56), 30.578,40.534,87.354,96.692, \\
111.696,131.478(\times 2), 165.365(\times 2), 217.116,252.738,259.934,280.677, \\
301.488(\times 2), 356.755(\times 2), 364.395,414.906,415.620,434.635\end{array}$ \\
\hline
\end{tabular}




\begin{tabular}{|c|c|}
\hline & $-17.244(\times 2),-16.727,-16.262(\times 2),-15.908,-15.492,-14.579(\times 2),-13.580$, \\
& $-13.499,-12.990,-11.934(\times 2),-9.733,-9.638,-8.787(\times 2),-2.270$, \\
$\mathcal{M}_{v} / g$ & $-2.200(\times 2),-1.682,-1.667,-1.589(\times 2),-0.992,-0.439(\times 2), 0.000(\times 72)$, \\
& $0.439(\times 2), 0.992,1.589(\times 2), 1.667,1.682,2.200(\times 2), 2.270,8.787(\times 2), 9.638$, \\
& $9.733,11.934(\times 2), 12.990,13.499,13.580,14.579(\times 2), 15.492,15.908,16.262(\times 2)$, \\
& $16.727,17.244(\times 2)$ \\
& $-11.544,-10.792(\times 2),-10.567,-10.381,-9.305(\times 2),-8.209,8.209,9.305(\times 2)$, \\
\hline \multirow{2}{*}{$A_{1} \quad$} & $10.381,10.567,10.792(\times 2), 11.544$ \\
& $-34.631,-32.377(\times 2),-31.701,-31.143,-27.915(\times 2),-24.628,-22.569$, \\
& $-20.442,-20.427(\times 2),-19.010(\times 2),-18.901,-18.521,-18.330,-15.874(\times 2)$, \\
& $-15.268,-14.769,-13.648(\times 2),-13.147,-12.018(\times 2),-11.722,-11.483(\times 2)$, \\
& $-11.300,-11.221,-8.511,-7.472(\times 2),-7.262,-7.234,-7.063,-6.994(\times 2)$, \\
& $-5.849(\times 2),-4.408,-4.258,-3.354(\times 2),-3.231,-2.922,-2.799,-2.595$, \\
& $-2.594(\times 2),-2.549,-2.445,-2.023(\times 2),-1.753,-1.362,-1.361,-0.967(\times 2)$, \\
& $-0.549,-0.432(\times 2),-0.159,0.159,0.432(\times 2), 0.549,0.967(\times 2), 1.361,1.362$, \\
& $1.753,2.023(\times 2), 2.445,2.549,2.594(\times 2), 2.595,2.799,2.922,3.231,3.354(\times 2)$, \\
& $4.258,4.408,5.849(\times 2), 6.994(\times 2), 7.063,7.234,7.262,7.472(\times 2), 8.511,11.221$, \\
& $11.300,11.483(\times 2), 11.722,12.018(\times 2), 13.147,13.648(\times 2), 14.769,15.268$, \\
& $15.874(\times 2), 18.330,18.521,18.901,19.010(\times 2), 20.427(\times 2), 20.442,22.569$, \\
& $24.628,27.915(\times 2), 31.143,31.701,32.377(\times 2), 34.631$
\end{tabular}

\begin{tabular}{|c|c|}
\hline \multicolumn{2}{|c|}{$R=2.694 \quad \Lambda=-94.909 \quad \operatorname{SUSY}=(0,0) \quad \operatorname{dim}(H)=0$} \\
\hline$\Psi_{128}$ & $\begin{array}{l}-0.183[15], 0.030[17], 0.914[26],-0.183[28], 0.000[32],-0.183[36],-0.030[38], \\
-0.914[45],-0.183[47],-0.274[52],-0.210[54], 0.000[58], 0.717[61], 0.232[63], \\
0.232[71], 0.717[73],-0.210[82],-0.274[84], 0.000[88], 0.089[\dot{1} \dot{5}], 0.007[\dot{1}] \text {, } \\
-0.007[\dot{2} \dot{6}],-0.089[\dot{2} \dot{8}],-0.089[\dot{3} \dot{6}], 1.148[\dot{3} \dot{8}],-1.148[\dot{4} \dot{5}], 0.089[\dot{4} \dot{7}],-0.189[\dot{5} \dot{2}], \\
-0.189[\dot{6} \dot{3}], 1.155[\dot{7} \dot{1}],-0.014[\dot{8} \dot{4}] ; \Delta=0.001376\end{array}$ \\
\hline $\mathcal{M}_{s} / g^{2}$ & $\begin{array}{l}-122.159,-101.902(\times 3),-99.564(\times 3),-92.320,-91.114(\times 3),-89.594, \\
-84.679(\times 3), \quad-81.085(\times 3),-67.603(\times 2),-65.087(\times 3),-60.114(\times 2), \\
-57.070(\times 3),-56.991,-56.302(\times 3),-51.834,-51.633(\times 2),-48.853(\times 3), \\
-34.254(\times 3),-29.201,-29.200(\times 2),-24.829(\times 2),-24.828,-22.066(\times 2), \\
-15.366,0.000(\times 56), 26.021,44.325(\times 2), 75.286(\times 2), 118.421,118.422(\times 2), \\
188.957(\times 3), \quad 219.164(\times 2), 272.548(\times 2), 272.549, \quad 390.285(\times 2), 390.286, \\
399.404(\times 2), 419.084\end{array}$ \\
\hline $\mathcal{M}_{v} / g$ & 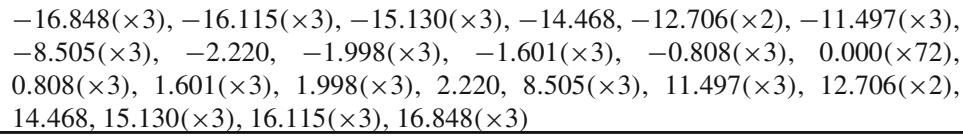 \\
\hline$A_{1}$ & $-10.933(\times 3),-10.908(\times 2),-8.708(\times 3), 8.708(\times 3), 10.908(\times 2), 10.933(\times 3)$ \\
\hline$A_{3}$ & $\begin{array}{l}-32.799(\times 3),-32.724(\times 2),-26.123(\times 3),-22.197,-20.350(\times 3),-19.056(\times 3), \\
-18.249(\times 2),-16.054(\times 3),-13.941(\times 3),-13.282,-11.579(\times 3),-10.849(\times 3), \\
-10.031,-7.648(\times 3),-7.585(\times 2),-6.763(\times 3),-5.620(\times 3),-4.518, \\
-3.652(\times 3),-2.942(\times 2),-2.669(\times 3),-2.626(\times 3),-2.625,-1.673(\times 2), \\
-1.598,-1.369(\times 3),-0.327(\times 3), 0.327(\times 3), 1.369(\times 3), 1.598,1.673(\times 2), \\
2.625,2.626(\times 3), 2.669(\times 3), 2.942(\times 2), 3.652(\times 3), 4.518,5.620(\times 3), 6.763(\times 3), \\
7.585(\times 2), 7.648(\times 3), 10.031,10.849(\times 3), 11.579(\times 3), 13.282,13.941(\times 3), \\
16.054(\times 3), 18.249(\times 2), 19.056(\times 3), 20.350(\times 3), 22.197,26.123(\times 3), 32.724(\times 2), \\
32.799(\times 3)\end{array}$ \\
\hline
\end{tabular}




\begin{tabular}{|c|c|}
\hline \multicolumn{2}{|c|}{$R=2.702 \quad \Lambda=-98.908 \quad \operatorname{SUSY}=(0,0) \quad \operatorname{dim}(H)=2$} \\
\hline$\Psi_{128}$ & 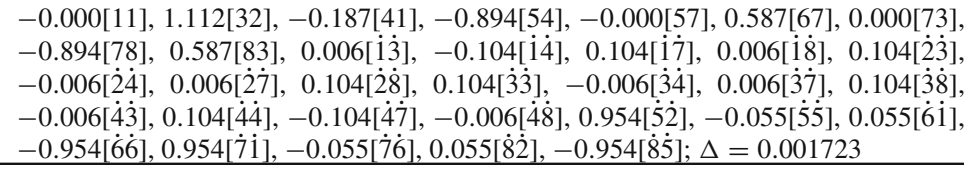 \\
\hline $\mathcal{M}_{s} / g^{2}$ & 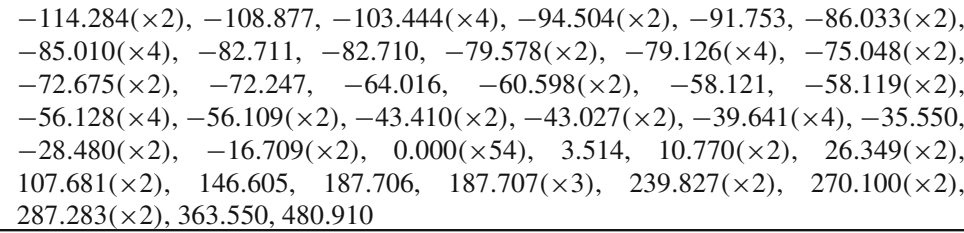 \\
\hline $\mathcal{M}_{v} / g$ & $\begin{array}{l}-16.938(\times 2),-16.390(\times 2),-15.841,-15.495(\times 2),-13.661(\times 2),-12.783, \\
-12.077(\times 2),-10.191(\times 2),-6.701(\times 2),-4.960(\times 2),-4.054(\times 2),-3.544(\times 2), \\
-2.367(\times 2),-1.986,-0.421(\times 2), 0.000(\times 74), 0.421(\times 2), 1.986,2.367(\times 2), \\
3.544(\times 2), 4.054(\times 2), 4.960(\times 2), 6.701(\times 2), 10.191(\times 2), 12.077(\times 2), 12.783, \\
13.661(\times 2), 15.495(\times 2), 15.841,16.390(\times 2), 16.938(\times 2)\end{array}$ \\
\hline$A_{1}$ & $\begin{array}{l}-12.861,-11.277(\times 2),-9.816(\times 2),-7.361(\times 2),-6.883,6.883,7.361(\times 2), \\
9.816(\times 2), 11.277(\times 2), 12.861\end{array}$ \\
\hline$A_{3}$ & $\begin{array}{l}-38.584,-33.831(\times 2),-29.449(\times 2),-22.082(\times 2),-20.649,-20.084(\times 2), \\
-19.869,-19.449(\times 2),-18.574(\times 2),-15.281(\times 2),-13.864,-12.272(\times 2), \\
-12.185(\times 2),-11.649(\times 2),-10.789(\times 2),-10.497(\times 2),-9.626(\times 2),-9.494, \\
-9.273(\times 2),-9.014(\times 2),-6.566(\times 2),-3.668(\times 2),-3.493(\times 2),-2.955(\times 2), \\
-2.940(\times 2),-2.557,-2.185(\times 2),-1.752(\times 2),-1.345(\times 2),-1.339(\times 2), \\
-1.152(\times 2),-1.133,-0.856(\times 2),-0.803(\times 2),-0.594,-0.371(\times 2), 0.371(\times 2), \\
0.594,0.803(\times 2), 0.856(\times 2), 1.133,1.152(\times 2), 1.339(\times 2), 1.345(\times 2), 1.752(\times 2), \\
2.185(\times 2), 2.557,2.940(\times 2), 2.955(\times 2), 3.493(\times 2), 3.668(\times 2), 6.566(\times 2), \\
9.014(\times 2), 9.273(\times 2), 9.494,9.626(\times 2), 10.497(\times 2), 10.789(\times 2), 11.649(\times 2), \\
12.185(\times 2), 12.272(\times 2), 13.864,15.281(\times 2), 18.574(\times 2), 19.449(\times 2), 19.869, \\
20.084(\times 2), 20.649,22.082(\times 2), 29.449(\times 2), 33.831(\times 2), 38.584\end{array}$ \\
\hline
\end{tabular}

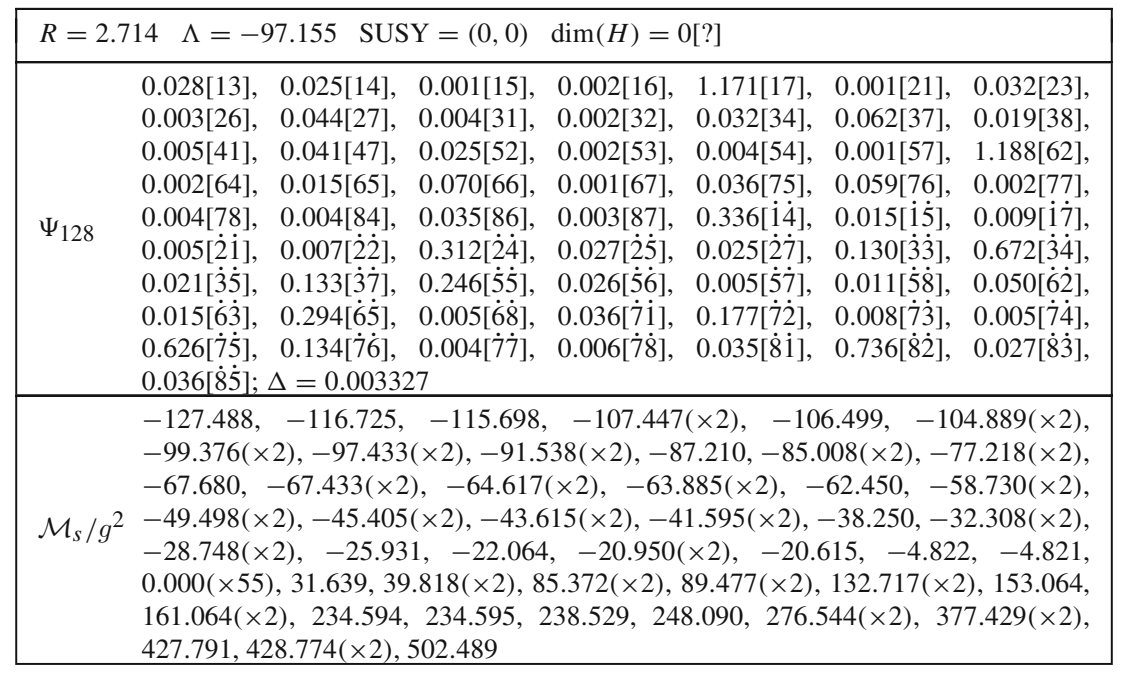




\begin{tabular}{|c|c|}
\hline $\mathcal{M}_{v} / g$ & $\begin{array}{l}-18.016, \quad-17.262(\times 2),-16.348, \quad-16.095(\times 2), \quad-15.975, \quad-14.663(\times 2), \\
-13.575,-13.546(\times 2),-12.234(\times 2),-12.122(\times 2),-7.992(\times 2),-1.854(\times 2), \\
-1.818(\times 2), \quad-1.806,-1.229(\times 2), \quad-1.158(\times 2), \quad 0.000(\times 74), \quad 1.158(\times 2), \\
1.229(\times 2), 1.806,1.818(\times 2), 1.854(\times 2), 7.992(\times 2), 12.122(\times 2), 12.234(\times 2), \\
13.546(\times 2), 13.575,14.663(\times 2), 15.975,16.095(\times 2), 16.348,17.262(\times 2), 18.016\end{array}$ \\
\hline$A_{1}$ & $\begin{array}{l}-11.563(\times 2),-10.605,-10.545(\times 2),-9.793(\times 2),-8.286,8.286,9.793(\times 2), \\
10.545(\times 2), 10.605,11.563(\times 2)\end{array}$ \\
\hline$A_{3}$ & $\begin{array}{l}-34.690(\times 2),-31.814,-31.634(\times 2),-29.378(\times 2),-24.859,-22.533, \\
-20.756(\times 2),-20.245,-19.461(\times 2),-19.377(\times 2),-19.046,-17.839(\times 2), \\
-16.534,-15.371(\times 2),-13.559(\times 2),-10.830(\times 2),-10.511(\times 2),-10.343, \\
-10.160(\times 2),-8.523(\times 2),-7.509(\times 2),-7.375(\times 2),-7.104,-6.368(\times 2), \\
-5.975,-5.974,-5.490(\times 2),-4.606(\times 2),-4.344(\times 2),-3.796,-3.361, \\
-3.160,-3.106(\times 2),-2.701(\times 2),-2.556(\times 2),-1.875(\times 2),-1.837(\times 2), \\
-0.971,-0.938(\times 2), 0.938(\times 2), 0.971,1.837(\times 2), 1.875(\times 2), 2.556(\times 2), \\
2.701(\times 2), 3.106(\times 2), 3.160,3.361,3.796,4.344(\times 2), 4.606(\times 2), 5.490(\times 2), \\
5.974,5.975,6.368(\times 2), 7.104,7.375(\times 2), 7.509(\times 2), 8.523(\times 2), 10.160(\times 2), \\
10.343,10.511(\times 2), 10.830(\times 2), 13.559(\times 2), 15.371(\times 2), 16.534,17.839(\times 2), \\
19.046,19.377(\times 2), 19.461(\times 2), 20.245,20.756(\times 2), 22.533,24.859,29.378(\times 2), \\
31.634(\times 2), 31.814,34.690(\times 2)\end{array}$ \\
\hline
\end{tabular}

\begin{tabular}{|c|c|}
\hline \multicolumn{2}{|c|}{$R=2.765 \quad \Lambda=-105.528 \quad \operatorname{SUSY}=(1,1) \quad \operatorname{dim}(H)=8$} \\
\hline$\Psi_{128}$ & $\begin{array}{l}0.430[\dot{1} \dot{1}], \quad 0.430[\dot{2} \dot{2}], \quad 0.037[\dot{3} \dot{3}], \quad 1.164[\dot{3} \dot{5}], \quad 0.430[\dot{4} \dot{4}], \quad 1.164[\dot{5} \dot{3}], \quad 0.037[\dot{5} \dot{5}], \\
0.430[\dot{6} \dot{6}], 0.430[\dot{7} \dot{7}], 0.430[\dot{8} \dot{8}] ; \Delta=0.001601\end{array}$ \\
\hline $\mathcal{M}_{s} / g^{2}$ & $\begin{array}{lllll}-105.528(\times 8), & -104.836(\times 12), & -99.646, \quad-84.488(\times 6), & -81.968(\times 8), \\
-73.200(\times 6), & -43.945, \quad-16.399(\times 12), \quad 0.000(\times 48), & 93.725, & 123.284(\times 8), \\
168.195(\times 12), & 220.267,235.669,489.263,696.633,720.700 & \end{array}$ \\
\hline $\mathcal{M}_{v} / g$ & $\begin{array}{lllll}-18.050, & -16.545(\times 6), \quad-15.958(\times 6), & -7.777, & -5.686(\times 6), & 0.000(\times 88), \\
5.686(\times 6), 7.777,15.958(\times 6), 16.545(\times 6), 18.050 & & \\
\end{array}$ \\
\hline$A_{1}$ & $-12.913,-10.822(\times 6),-5.136,5.136,10.822(\times 6), 12.913$ \\
\hline$A_{3}$ & $\begin{array}{l}-38.740,-32.466(\times 6),-23.608,-23.186,-21.681(\times 6),-19.252,-12.913, \\
-11.408(\times 6),-10.822(\times 6),-9.990(\times 8),-5.136(\times 8),-4.304(\times 12),-2.711, \\
-0.549(\times 6), 0.549(\times 6), 2.711,4.304(\times 12), 5.136(\times 8), 9.990(\times 8), 10.822(\times 6), \\
11.408(\times 6), 12.913,19.252,21.681(\times 6), 23.186,23.608,32.466(\times 6), 38.740\end{array}$ \\
\hline
\end{tabular}

\begin{tabular}{|c|c|}
\hline \multicolumn{2}{|c|}{$R=2.794 \quad \Lambda=-110.183 \quad \operatorname{SUSY}=(0,0) \quad \operatorname{dim}(H)=0$} \\
\hline$\Psi_{128}$ & 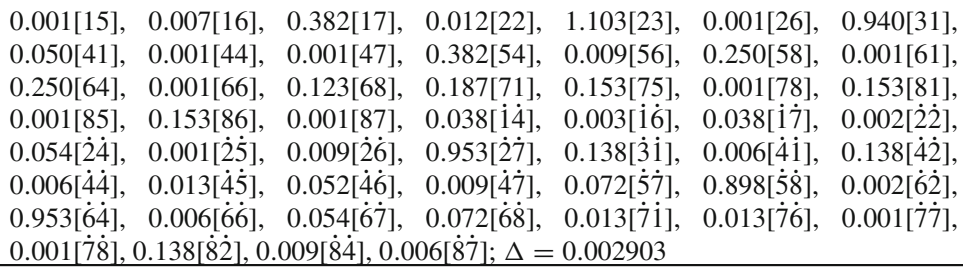 \\
\hline $\mathcal{M}_{s} / g^{2}$ & $\begin{array}{l}-133.015,-117.293,-115.625,-113.736,-111.312(\times 2),-110.001,-102.968, \\
-98.982,-98.828(\times 2),-96.352,-95.427(\times 2),-90.532,-89.376(\times 2),-87.597, \\
-86.431,-85.404,-85.403,-83.935,-81.278,-80.405,-77.506(\times 2),-72.287, \\
-70.695,-63.676,-62.309(\times 2),-58.694(\times 2),-56.688,-55.152(\times 2),-52.808, \\
-51.727,-45.520(\times 2),-45.281,-45.019(\times 2),-43.825,-43.660(\times 2),-43.104, \\
-21.391,0.000(\times 56), 5.484,7.608,9.683,24.616,34.500,84.402,155.280, \\
176.510,176.511,198.333(\times 2), 238.258,267.065,299.355,353.261(\times 2), 376.012, \\
377.434(\times 2), 386.834,389.143,489.376,509.847,519.205\end{array}$ \\
\hline
\end{tabular}




\begin{tabular}{|c|c|c|}
\hline & $-21.524,-19.834,-19.009,-17.182,-16.944,-16.563,-15.860,-15.743$, \\
& $-15.101,-14.172,-14.006,-13.291,-13.121,-12.903,-12.639,-10.237$, \\
$\mathcal{M}_{v} / g$ & $-7.161,-6.198,-5.877,-5.828,-4.268,-2.671,-2.664,-2.503,-1.621$, \\
& $-0.946,-0.822,-0.394,0.000(\times 72), 0.394,0.822,0.946,1.621,2.503,2.664,2.671$, \\
& $4.268,5.828,5.877,6.198,7.161,10.237,12.639,12.903,13.121,13.291,14.006$, \\
& $14.172,15.101,15.743,15.860,16.563,16.944,17.182,19.009,19.834,21.524$ \\
\hline \multirow{2}{*}{$A_{1}$} & $-13.917(\times 2),-11.836(\times 2),-8.942(\times 2),-8.728(\times 2), 8.728(\times 2), 8.942(\times 2)$, \\
\hline & $11.836(\times 2), 13.917(\times 2)$ \\
& $-41.752(\times 2), \quad-35.509(\times 2), \quad-26.825(\times 2), \quad-26.184(\times 2), \quad-23.207(\times 2)$, \\
& $-22.624(\times 2), \quad-21.743(\times 2), \quad-20.976(\times 2), \quad-18.529(\times 2), \quad-16.600(\times 2)$, \\
& $-15.521(\times 2), \quad-13.736(\times 2),-12.955(\times 2), \quad-12.150(\times 2), \quad-11.518(\times 2)$, \\
& $-10.833(\times 2),-10.159(\times 2),-8.331(\times 2),-6.723(\times 2),-6.708(\times 2),-5.488(\times 2)$, \\
& $-5.447(\times 2),-4.135(\times 2),-3.583(\times 2),-2.702(\times 2),-2.599(\times 2),-1.869(\times 2)$, \\
& $-1.261(\times 2),-1.197(\times 2),-0.758(\times 2),-0.502(\times 2),-0.171(\times 2), 0.171(\times 2)$, \\
& $0.502(\times 2), 0.758(\times 2), 1.197(\times 2), 1.261(\times 2), 1.869(\times 2), 2.599(\times 2), 2.702(\times 2)$, \\
& $3.583(\times 2), 4.135(\times 2), 5.447(\times 2), 5.488(\times 2), 6.708(\times 2), 6.723(\times 2), 8.331(\times 2)$, \\
& $10.159(\times 2), \quad 10.833(\times 2), 11.518(\times 2), 12.150(\times 2), 12.955(\times 2), 13.736(\times 2)$, \\
& $15.521(\times 2), 16.600(\times 2), 18.529(\times 2), 20.976(\times 2), 21.743(\times 2), 22.624(\times 2)$, \\
& $23.207(\times 2), 26.184(\times 2), 26.825(\times 2), 35.509(\times 2), 41.752(\times 2)$
\end{tabular}

\begin{tabular}{|c|c|}
\hline \multicolumn{2}{|c|}{$R=2.930 \quad \Lambda=-129.687 \quad \operatorname{SUSY}=(0,0) \quad \operatorname{dim}(H)=0$} \\
\hline$\Psi_{128}$ & 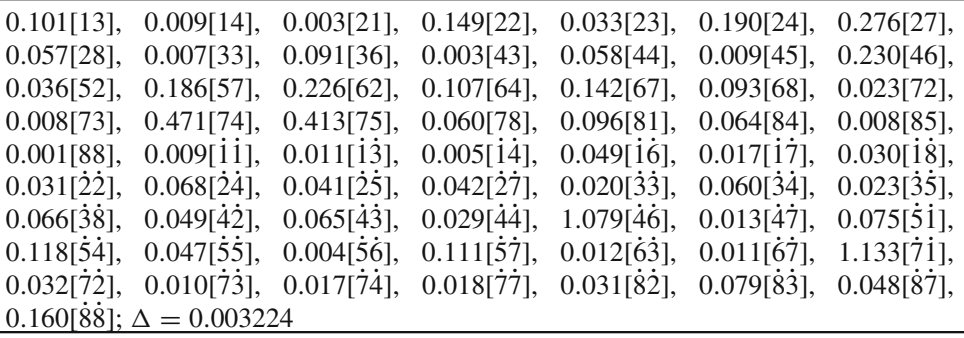 \\
\hline $\mathcal{M}_{s} / g^{2}$ & $\begin{array}{l}-167.192,-155.200,-155.094,-148.093,-146.482,-137.914,-137.577, \\
-129.172,-123.484,-122.772,-122.187,-120.683,-115.148,-112.250, \\
-107.441,-107.381,-101.718,-97.666,-95.019,-93.027,-86.685,-85.145, \\
-82.214,-79.335,-76.147,-73.895,-73.293,-72.928,-71.376,-67.770, \\
-67.297,-62.750,-61.535,-58.960,-54.374,-51.650,-46.267,-44.790, \\
-42.974,-37.874,-34.164,-28.963,-18.963,-4.669,0.000(\times 56), 0.193,7.449, \\
38.722,56.621,57.214,106.140,116.404,120.791,173.357,177.396,179.313, \\
182.565,242.766,264.806,285.395,295.517,305.174,336.581,361.974,363.457, \\
371.873,393.882,498.092,527.847,538.051,601.157,601.524,656.846\end{array}$ \\
\hline $\mathcal{M}_{v} / g$ & $\begin{array}{l}-21.618,-20.639,-20.384,-19.622,-19.259,-19.004,-18.956,-17.919, \\
-17.173,-16.938,-16.103,-15.342,-15.082,-14.718,-14.067,-13.082, \\
-11.303,-6.402,-5.613,-5.609,-5.195,-4.048,-3.006,-2.252,-1.856, \\
-1.409,-1.263,-1.103,0.000(\times 72), 1.103,1.263,1.409,1.856,2.252,3.006,4.048, \\
5.195,5.609,5.613,6.402,11.303,13.082,14.067,14.718,15.082,15.342,16.103, \\
16.938,17.173,17.919,18.956,19.004,19.259,19.622,20.384,20.639,21.618\end{array}$ \\
\hline$A_{1}$ & $\begin{array}{l}-15.214,-15.213,-12.691,-12.121,-12.112,-11.288,-9.742,-8.803,8.803, \\
9.742,11.288,12.112,12.121,12.691,15.213,15.214\end{array}$ \\
\hline
\end{tabular}




\begin{tabular}{|c|c|}
\hline$A_{3}$ & $\begin{array}{l}-45.642,-45.639,-38.073,-36.363,-36.335,-33.864,-29.225,-26.410, \\
-24.827,-24.613,-24.058,-24.003,-23.667,-23.660,-23.231,-22.894, \\
-22.731,-22.359,-20.004,-19.589,-18.827,-16.558,-16.401,-15.802, \\
-15.439,-15.394,-14.576,-13.956,-13.032,-13.027,-12.334,-12.277, \\
-11.570,-10.991,-10.424,-10.247,-9.649,-8.891,-7.585,-7.277,-6.978, \\
-6.584,-6.426,-5.918,-5.421,-5.221,-5.104,-4.877,-4.785,-4.450, \\
-4.257,-4.038,-4.037,-3.454,-3.145,-2.814,-2.473,-2.317,-2.295, \\
-2.221,-2.070,-2.056,-1.493,-1.267,1.267,1.493,2.056,2.070,2.221,2.295, \\
2.317,2.473,2.814,3.145,3.454,4.037,4.038,4.257,4.450,4.785,4.877,5.104, \\
5.221,5.421,5.918,6.426,6.584,6.978,7.277,7.585,8.891,9.649,10.247,10.424, \\
10.991,11.570,12.277,12.334,13.027,13.032,13.956,14.576,15.394,15.439, \\
15.802,16.401,16.558,18.827,19.589,20.004,22.359,22.731,22.894,23.231, \\
23.660,23.667,24.003,24.058,24.613,24.827,26.410,29.225,33.864,36.335, \\
36.363,38.073,45.639,45.642\end{array}$ \\
\hline
\end{tabular}

\begin{tabular}{|c|c|}
\hline \multicolumn{2}{|c|}{$R=2.940 \quad \Lambda=-131.996 \quad \operatorname{SUSY}=(0,0) \quad \operatorname{dim}(H)=0$} \\
\hline$\Psi_{128}$ & $\begin{array}{lllllll}0.009[15], & 0.058[16], & 0.468[17], & 0.057[21], & 0.449[23], & 0.078[27], & 0.301[33], \\
0.001[35], & 0.104[36], & 0.065[44], & 1.023[46], & 0.009[48], & 0.415[51], & 0.301[54], \\
0.104[55], & 0.001[56], & 0.017[64], & 0.058[68], & 0.134[71], & 0.025[72], & 0.058[75], \\
0.009[76], & 0.468[78], & 0.301[81], & 0.449[84], & 0.078[88], & 0.079[\dot{1} \dot{4}], & 0.208[\dot{1} \dot{8}], \\
0.020[\dot{2} \dot{5}], & 0.044[\dot{3} \dot{1}], & 0.001[\dot{3} \dot{6}], & 0.034[\dot{3} \dot{7}], & 0.020[\dot{4} \dot{4}], & 0.922[\dot{4} \dot{5}], & 0.079[\dot{4} \dot{8}], \\
1.030[\dot{5} \dot{2}], & 0.069[\dot{5} \dot{8}], & 0.001[\dot{6} \dot{1}], & 0.054[\dot{6} \dot{3}], & 0.132[\dot{6} \dot{6}], & 0.033[\dot{6} 7], & 0.034[\overline{7} \dot{1}], \\
0.033[\dot{7} \dot{6}], & 0.026[\dot{8} \dot{1}], 0.033[\dot{8} \dot{3}], 0.053[\dot{8} \dot{7}] ; \Delta=0.002539 & & \\
\end{array}$ \\
\hline $\mathcal{M}_{s} / g^{2}$ & $\begin{array}{l}-167.789,-160.012,-152.037,-151.218,-146.804,-142.342,-141.392, \\
-129.648,-127.458,-124.191,-121.761,-120.720,-113.697,-111.125, \\
-108.256,-107.565,-107.203,-105.111,-96.798,-96.515,-95.424,-94.499, \\
-90.414,-86.739,-84.720,-74.863,-73.257,-72.680,-69.820,-68.793, \\
-64.039,-63.079,-61.121,-60.869,-57.972,-57.434,-53.590,-52.779, \\
-52.605,-40.282,-39.977,-30.601,-18.838,-10.241,-2.589,0.000(\times 56), \\
30.876,32.011,35.794,47.087,81.767,102.099,105.940,152.854,156.832,191.620, \\
217.279,270.901,289.097,294.233,303.499,310.195,311.218,318.183,360.771, \\
372.630,400.411,487.363,533.051,561.596,576.078,617.812,630.527\end{array}$ \\
\hline $\mathcal{M}_{v / g}$ & $\begin{array}{l}-21.662,-20.808,-20.779,-20.399,-19.940,-18.612,-18.461,-17.824, \\
-17.626,-17.218,-16.467,-14.595,-14.533,-14.089,-13.724,-13.189, \\
-10.952,-6.851,-5.749,-5.449,-4.792,-4.568,-2.360,-2.159,-1.784, \\
-1.630,-1.110,-0.967,0.000(\times 72), 0.967,1.110,1.630,1.784,2.159,2.360,4.568, \\
4.792,5.449,5.749,6.851,10.952,13.189,13.724,14.089,14.533,14.595,16.467, \\
17.218,17.625,17.824,18.461,18.612,19.940,20.399,20.779,20.808,21.662\end{array}$ \\
\hline$A_{1}$ & $\begin{array}{l}-16.216,-15.479,-12.530,-11.752,-11.360,-11.338,-9.269,-9.207,9.207, \\
9.269,11.338,11.360,11.752,12.530,15.479,16.216\end{array}$ \\
\hline$A_{3}$ & $\begin{array}{l}-48.647,-46.436,-37.589,-35.255,-34.079,-34.014,-27.808,-27.620, \\
-25.280,-24.790,-24.140,-23.995,-23.796,-23.305,-23.168,-22.928, \\
-22.609,-21.836,-20.066,-19.857,-18.384,-17.352,-15.945,-15.844, \\
-15.169,-14.974,-14.134,-14.030,-13.635,-13.509,-12.849,-12.760, \\
-12.046,-11.599,-9.432,-9.378,-9.057,-8.770,-7.932,-7.656,-7.125, \\
-7.029,-6.580,-6.570,-5.476,-5.365,-4.844,-4.434,-4.208,-4.057, \\
-3.802,-3.276,-3.228,-3.006,-2.762,-2.294,-2.101,-1.962,-1.726, \\
-1.625,-1.421,-1.404,-1.012,-0.590,0.590,1.012,1.404,1.421,1.625,1.726, \\
1.962,2.101,2.294,2.762,3.006,3.228,3.276,3.802,4.057,4.208,4.434,4.844, \\
5.365,5.476,6.570,6.580,7.029,7.125,7.656,7.932,8.770,9.057,9.378,9.432, \\
11.599,12.046,12.760,12.849,13.509,13.635,14.030,14.134,14.974,15.169, \\
15.844,15.945,17.352,18.384,19.857,20.066,21.836,22.609,22.928,23.168, \\
23.305,23.796,23.995,24.140,24.790,25.280,27.620,27.808,34.014,34.079, \\
35.255,37.589,46.436,48.647\end{array}$ \\
\hline
\end{tabular}




\begin{tabular}{|c|c|}
\hline \multicolumn{2}{|c|}{$R=2.994 \quad \Lambda=-139.990 \quad \mathrm{SUSY}=(0,0) \quad \operatorname{dim}(H)=0$} \\
\hline$\Psi_{128}$ & 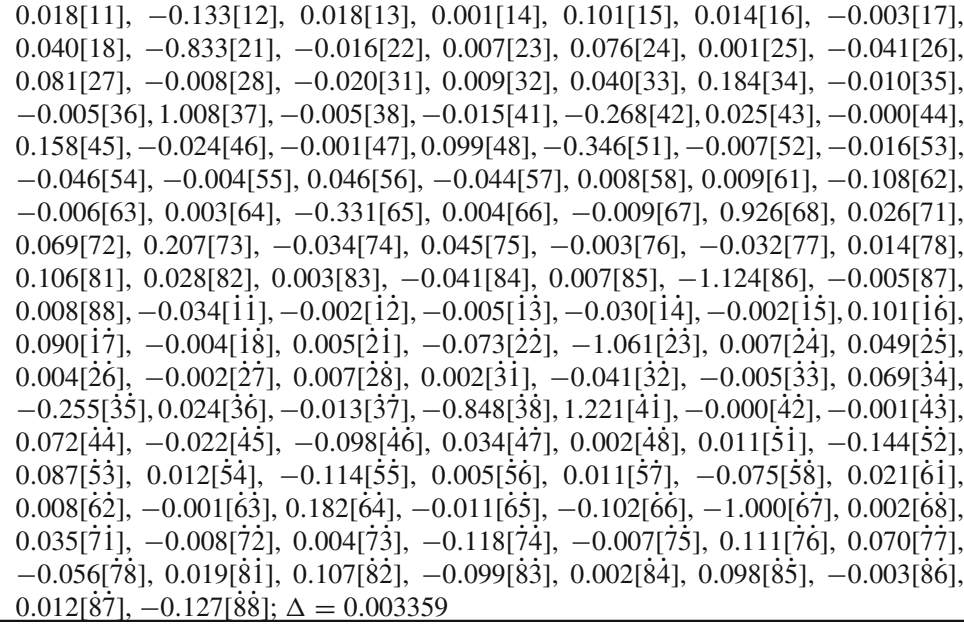 \\
\hline $\mathcal{M}_{s} / g^{2}$ & $\begin{array}{l}-170.661,-169.131,-157.214,-152.580,-143.638,-141.938,-138.884, \\
-137.723,-133.828,-128.725,-126.386,-123.969,-117.277,-114.491, \\
-113.402,-110.453,-108.343,-103.767,-103.644,-98.387,-94.484,-92.595, \\
-90.156,-86.134,-81.194,-78.556,-74.184,-70.460,-68.030,-64.520, \\
-62.432,-59.503,-55.843,-54.204,-51.887,-50.959,-46.236,-44.405, \\
-41.040,-38.653,-35.884,-20.773,-6.480,0.000(\times 56), 8.735,35.183,59.093, \\
83.130,106.368,129.941,164.395,170.710,177.994,215.628,237.853,259.472, \\
271.662,289.195,303.006,305.219,382.784,409.064,422.330,425.019,490.913, \\
506.625,518.517,536.365,571.218,618.395,641.294,680.369,715.491\end{array}$ \\
\hline $\mathcal{M}_{v} / g$ & $\begin{array}{l}-23.925,-22.868,-21.820,-21.091,-20.772,-19.928,-19.446,-18.243, \\
-17.711,-17.410,-17.304,-17.002,-15.699,-15.532,-15.122,-14.698, \\
-13.032,-10.344,-7.800,-7.137,-5.117,-4.322,-3.646,-3.468,-2.217, \\
-1.707,-1.387,-0.947,0.000(\times 72), 0.880,1.325,1.433,1.831,2.416,3.795,4.308, \\
5.158,6.748,9.060,12.604,13.124,14.268,15.194,16.165,16.390,16.616,16.997, \\
17.188,17.560,18.258,19.789,20.019,20.466,20.569,21.407,21.921,24.208\end{array}$ \\
\hline$A_{1}$ & $572,-10.374,-10.362,9.605$, \\
\hline$A_{3}$ & $\begin{array}{l}-51.099,-44.590,-42.721,-39.662,-37.322,-34.717,-31.121,-31.085, \\
-27.529,-26.741,-26.530,-25.660,-25.003,-24.651,-24.304,-23.740, \\
-23.454,-23.216,-22.563,-21.374,-19.865,-19.643,-18.805,-17.560, \\
-17.047,-16.864,-16.038,-15.212,-14.853,-14.262,-14.005,-13.344, \\
-13.155,-12.737,-12.296,-11.966,-11.131,-10.961,-10.340,-10.151, \\
-8.850,-8.173,-6.562,-6.342,-5.999,-5.373,-5.153,-4.919,-4.627, \\
-4.433,-4.313,-4.046,-3.730,-3.360,-3.112,-2.871,-2.692,-2.249, \\
-1.920,-1.603,-1.296,-1.096,-0.700,-0.678,-0.338,0.736,1.019,1.338, \\
1.839,2.052,2.344,2.592,3.309,3.590,3.735,4.156,4.258,4.398,4.636,4.932, \\
5.112,5.387,5.499,5.769,6.474,6.660,7.239,8.734,9.749,10.106,10.897,11.101, \\
11.285,11.405,12.498,12.688,13.688,13.875,14.248,15.397,15.759,16.073, \\
16.415,17.465,17.776,18.814,19.822,20.518,22.300,22.896,23.348,23.530, \\
23.707,23.958,24.764,24.819,25.198,25.830,26.685,27.014,28.816,32.954, \\
36.062,38.532,40.600,41.695,45.473,48.185\end{array}$ \\
\hline
\end{tabular}




\begin{tabular}{|c|c|}
\hline \multicolumn{2}{|c|}{$R=3.073 \quad \Lambda=-154.840 \quad \operatorname{SUSY}=(0,0) \quad \operatorname{dim}(H)=0$} \\
\hline$\Psi_{128}$ & 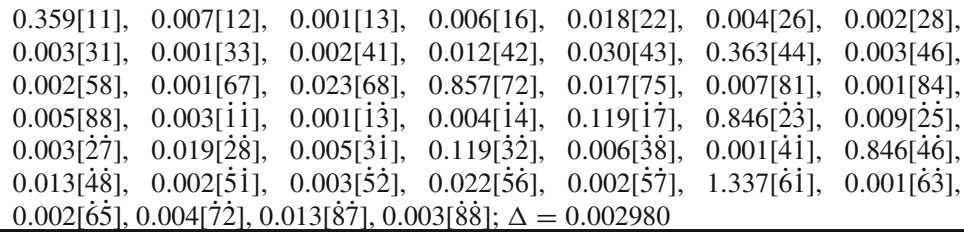 \\
\hline $\mathcal{M}_{s} / g^{2}$ & $\begin{array}{l}-198.545,-196.039,-196.034,-180.314,-173.847,-149.172,-142.776, \\
-139.931,-139.774,-138.540,-138.240,-137.231,-137.082,-125.158, \\
-123.864,-123.092,-121.947,-121.748,-93.677,-81.416,-73.772,-69.650, \\
-69.408,-69.194,-68.844,-55.595,-55.554,-55.303,-52.240,-50.919, \\
-32.618,-31.930,-31.762,-31.484,-21.530,-19.649,-19.384,-16.694, \\
-1.006,-0.702,0.000(\times 56), 0.003,33.671,66.032,66.123,86.310,181.163, \\
192.468,207.376,208.377,213.462,219.756,247.967,247.997,349.145,363.102, \\
377.506,465.875,465.982,468.594,469.213,519.525,524.242,564.885,619.868, \\
619.913,621.333,622.058,707.580,1091.118,1105.724,1193.978,1210.310\end{array}$ \\
\hline $\mathcal{M}_{v} / g$ & $\begin{array}{l}-25.856,-25.804,-25.745,-25.730,-24.728,-23.686,-22.927,-19.598, \\
-19.393,-19.204,-19.182,-19.084,-18.973,-18.768,-16.570,-13.696, \\
-13.670,-13.650,-13.638,-10.208,-3.299(\times 2),-2.014,-2.013,-2.006, \\
-1.993,-1.718,-0.129,0.000(\times 72), 0.129,1.719,1.988,2.008,2.012,2.017, \\
3.299(\times 2), 10.208,13.642,13.656,13.664,13.693,16.570,18.790,18.912,19.106, \\
19.176,19.267,19.347,19.604,22.927,23.686,24.729,25.733,25.742,25.793, \\
25.868\end{array}$ \\
\hline$A_{1}$ & $\begin{array}{l}-17.092,-17.039,-16.005,-15.917,-13.999,-13.976,-11.586(\times 2), 11.583, \\
11.589,13.970,14.005,15.955,15.966,17.064,17.069\end{array}$ \\
\hline$A_{3}$ & $\begin{array}{l}-51.277,-51.116,-48.016,-47.752,-41.997,-41.929,-35.627,-35.621, \\
-34.757(\times 2),-28.479,-28.467,-27.374,-27.361,-27.255,-27.250,-25.662, \\
-25.623,-25.240,-25.206,-24.536,-24.530,-22.108,-22.084,-21.718, \\
-21.698,-17.787,-17.748,-15.671,-15.653,-14.582,-14.581,-14.517, \\
-14.515,-13.385,-13.359,-12.917,-12.898,-10.975,-10.952,-10.855, \\
-10.854,-9.526,-9.413,-9.050,-8.983,-6.671,-6.660,-5.774,-5.749, \\
-5.267,-5.227,-3.730,-3.723,-3.142,-3.128,-2.551,-2.550,-2.502(\times 2), \\
-1.724,-1.715,-1.271,-1.256,1.227,1.311,1.663,1.764,2.502,2.503,2.551, \\
2.552,3.112,3.157,3.698,3.752,5.235,5.268,5.744,5.770,6.655,6.677,8.939, \\
9.126,9.371,9.534,10.853,10.854,10.960,10.970,12.876,12.949,13.307,13.429, \\
14.487(\times 2), 14.611,14.612,15.648,15.673,17.750,17.785,21.699,21.715,22.080, \\
22.113,24.531,24.535,25.220,25.226,25.631,25.653,27.227,27.291,27.344, \\
27.378,28.466,28.480,34.748,34.767,35.622,35.626,41.911,42.015,47.865, \\
47.898,51.191,51.208\end{array}$ \\
\hline
\end{tabular}

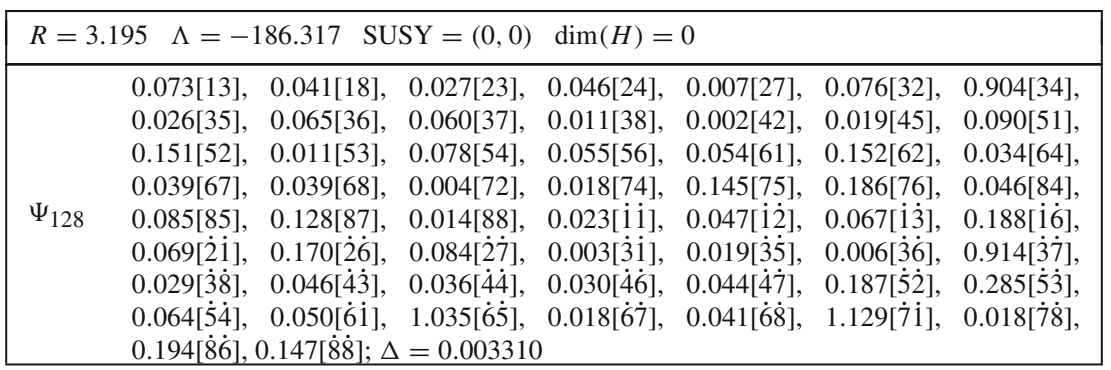




\begin{tabular}{|c|c|}
\hline $\mathcal{M}_{s} / g^{2}$ & $\begin{array}{l}-208.049,-194.511,-193.639,-192.445,-185.044,-179.848,-176.723, \\
-172.980,-172.498,-160.186,-159.011,-156.206,-145.870,-145.398, \\
-144.900,-141.393,-138.423,-137.463,-136.894,-118.901,-115.381, \\
-114.457,-107.287,-106.395,-103.057,-102.613,-96.612,-94.411,-87.786, \\
-85.740,-74.361,-71.480,-68.072,-66.757,-61.510,-59.718,-54.803, \\
-48.587,-44.893,-29.606,0.000(\times 56), 3.717,11.469,44.756,140.414,163.526, \\
163.864,170.272,177.239,214.098,260.016,262.924,283.394,312.542,367.648, \\
393.529,401.959,484.099,486.233,517.310,534.610,546.382,579.991,599.155, \\
638.941,639.599,678.692,698.977,699.425,796.201,844.973,857.259,901.831\end{array}$ \\
\hline $\mathcal{M}_{v} / g$ & $\begin{array}{l}-28.086,-25.662,-25.352,-24.914,-24.240,-23.355,-22.657,-22.623, \\
-21.101,-21.094,-19.984,-19.164,-18.707,-18.151,-17.545,-17.006, \\
-15.158,-13.004,-11.061,-9.756,-8.056,-6.981,-6.443,-3.752,-3.609, \\
-3.008,-2.509,-1.035,0.000(\times 72), 1.035,2.509,3.008,3.609,3.752,6.443,6.981, \\
8.056,9.756,11.061,13.004,15.158,17.006,17.545,18.151,18.707,19.164,19.984, \\
21.094,21.101,22.623,22.657,23.355,24.240,24.914,25.352,25.662,28.086\end{array}$ \\
\hline$A_{1}$ & $\begin{array}{l}-20.275,-18.422,-17.694,-14.535,-14.286,-14.082,-11.563,-10.559, \\
10.559,11.563,14.082,14.286,14.535,17.694,18.422,20.275\end{array}$ \\
\hline$A_{3}$ & $\begin{array}{l}-60.826,-55.266,-53.081,-43.605,-42.858,-42.246,-34.690,-31.677, \\
-31.310,-31.028,-29.556,-28.794,-28.650,-28.430,-27.841,-27.379, \\
-27.371,-26.952,-26.560,-25.100,-24.255,-23.313,-22.938,-22.583, \\
-21.456,-20.614,-20.139,-19.080,-18.297,-17.354,-17.099,-16.640, \\
-16.385,-15.510,-15.230,-15.127,-14.178,-13.778,-12.904,-11.967, \\
-11.350,-11.072,-10.609,-8.871,-8.287,-8.195,-7.733,-6.197,-5.242, \\
-5.103,-4.852,-4.576,-4.339,-4.029,-3.741,-3.309,-2.832,-2.372, \\
-1.912,-1.858,-1.404,-0.968,-0.376,-0.042,0.042,0.376,0.968,1.404,1.858, \\
1.912,2.372,2.832,3.309,3.741,4.029,4.339,4.576,4.852,5.103,5.242,6.197, \\
7.733,8.195,8.287,8.871,10.609,11.072,11.350,11.967,12.904,13.778,14.178, \\
15.127,15.230,15.510,16.385,16.640,17.099,17.354,18.297,19.080,20.139, \\
20.614,21.456,22.583,22.938,23.313,24.255,25.100,26.560,26.952,27.371, \\
27.379,27.841,28.430,28.650,28.794,29.556,31.028,31.310,31.677,34.690, \\
42.246,42.858,43.605,53.081,55.266,60.826\end{array}$ \\
\hline
\end{tabular}

\begin{tabular}{|c|c|}
\hline \multicolumn{2}{|c|}{$R=3.212 \quad \Lambda=-190.141 \quad \operatorname{SUSY}=(0,0) \quad \operatorname{dim}(H)=0$} \\
\hline$\Psi_{128}$ & 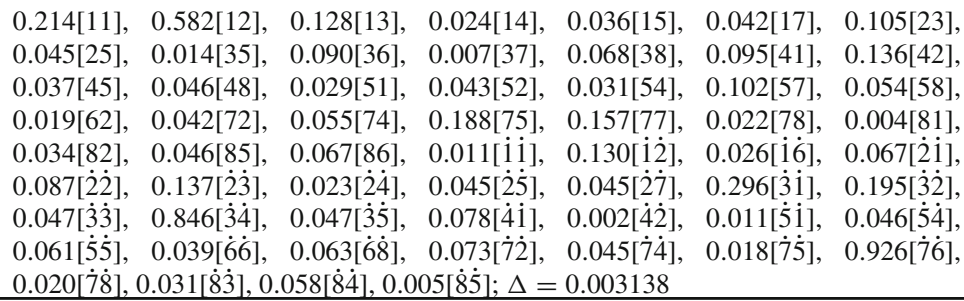 \\
\hline $\mathcal{M}_{s} / g^{2}$ & $\begin{array}{l}-220.803,-214.121,-204.396,-199.172,-192.642,-188.574,-184.707, \\
-182.009,-177.592,-171.574,-162.712,-158.371,-157.760,-151.484, \\
-147.917,-138.336,-136.891,-131.804,-124.372,-121.053,-120.713, \\
-116.663,-107.808,-104.931,-100.868,-97.301,-92.746,-87.345,-82.341, \\
-79.702,-74.869,-67.015,-63.980,-58.855,-53.863,-52.256,-42.128, \\
-29.704,-22.226,-18.988,0.000(\times 56), 13.841,35.934,120.452,126.906,152.185, \\
188.044,200.160,203.749,259.206,263.093,291.771,307.249,328.397,357.489, \\
385.087,412.253,423.885,470.096,489.830,526.124,568.378,606.239,627.825, \\
646.897,704.541,714.105,773.178,819.974,851.335,886.997,890.487,936.687\end{array}$ \\
\hline
\end{tabular}




\begin{tabular}{|c|c|}
\hline & $-28.473,-27.922,-25.895,-25.106,-24.649,-23.413,-23.344,-22.725$, \\
& $-22.171,-21.066,-20.711,-19.830,-18.941,-18.787,-18.162,-17.425$, \\
$\mathcal{M}_{v} / g$ & $-14.717,-13.264,-11.110,-8.283,-7.629,-6.894,-6.477,-4.246,-3.711$, \\
& $-2.624,-2.322,-1.194,0.000(\times 72), 0.750,2.172,2.814,3.186,3.746,6.368,6.587$, \\
& $7.329,10.764,11.962,14.029,15.540,17.770,18.205,18.928,19.662,20.197,20.832$, \\
& $21.178,21.740,22.494,22.751,23.482,23.843,24.253,25.350,27.250,27.905$ \\
\hline \multirow{2}{*}{$A_{1}$} & $-21.418,-19.137,-17.794,-14.901,-14.455,-13.192,-12.201,-10.260$, \\
\hline & $10.076,13.129,13.812,14.338,15.363,17.937,18.879,19.823$ \\
& $-64.254,-57.411,-53.382,-44.702,-43.364,-39.576,-36.603,-32.422$, \\
& $-32.114,-31.117,-30.779,-29.644,-29.278,-28.942,-27.966,-27.693$, \\
& $-27.581,-27.418,-26.954,-25.981,-25.126,-24.452,-23.050,-22.024$, \\
& $-21.117,-20.981,-20.659,-19.974,-18.996,-18.318,-17.686,-16.521$, \\
& $-16.491,-16.321,-15.632,-15.308,-14.433,-13.769,-13.672,-12.035$, \\
& $-11.507,-10.895,-9.816,-9.426,-9.116,-8.416,-7.720,-6.277,-5.513$, \\
& $-5.256,-4.812,-4.511,-4.417,-4.201,-3.895,-3.685,-3.032,-2.889$, \\
& $-2.596,-2.084,-1.736,-1.445,-1.089,-0.363,0.502,1.076,1.197,1.463,1.611$, \\
& $1.795,2.619,2.873,3.213,3.790,4.218,4.539,5.212,5.855,5.893,6.176,7.200$, \\
& $7.774,8.734,8.849,9.435,10.051,10.378,11.889,12.756,13.068,13.419,14.427$, \\
& $15.289,15.857,16.134,16.707,16.908,17.380,17.698,18.719,19.735,20.923$, \\
& $21.621,22.349,23.182,23.275,24.318,24.756,25.449,26.893,27.226,27.629$, \\
& $27.769,28.284,28.374,28.756,29.303,29.906,30.228,31.697,32.222,39.387$, \\
& $41.436,43.014,46.089,53.810,56.636,59.469$ \\
&
\end{tabular}

\begin{tabular}{|c|c|}
\hline \multicolumn{2}{|c|}{$R=3.324 \quad \Lambda=-217.963 \quad \operatorname{SUSY}=(0,0) \quad \operatorname{dim}(H)=0$} \\
\hline$\Psi_{128}$ & 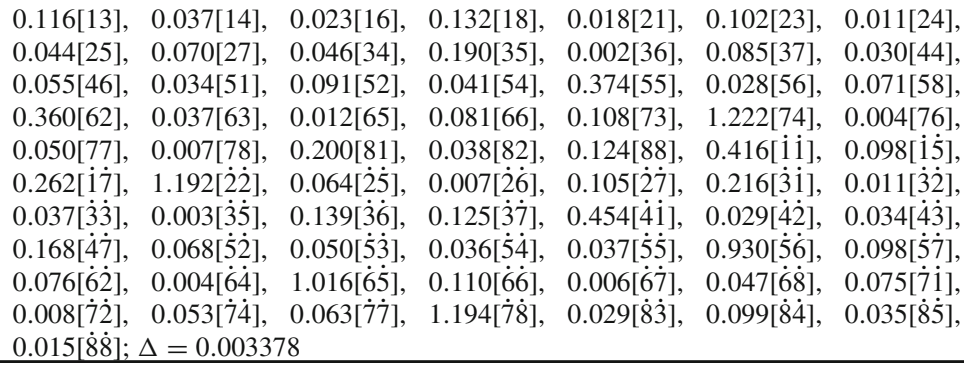 \\
\hline $\mathcal{M}_{s} / g^{2}$ & $\begin{array}{l}-294.572,-256.305,-250.669,-242.125,-238.330,-224.764,-217.509, \\
-209.937,-200.993,-181.259,-176.925,-172.595,-166.299,-161.759, \\
-152.604,-144.958,-133.267,-122.712,-120.235,-112.843,-109.449, \\
-105.444,-100.729,-92.939,-90.522,-87.742,-77.591,-70.690,-66.621, \\
-62.351,-55.139,-49.963,-42.385,-22.765,-13.256,0.000(\times 48), 0.001(\times 8), \\
26.806,59.434,72.972,127.102,154.608,165.387,188.199,229.840,243.718, \\
263.430,269.036,332.570,373.767,383.528,404.518,426.681,445.254,520.909, \\
549.360,558.077,594.432,627.851,661.050,683.281,721.004,767.009,793.838, \\
819.409,880.098,992.297,994.389,1015.828,1043.321,1090.918,1119.911, \\
1219.352,1250.636\end{array}$ \\
\hline $\mathcal{M}_{v} / g$ & $\begin{array}{l}-30.351,-30.043,-28.812,-27.836,-27.121,-26.525,-26.278,-25.428, \\
-24.431,-24.039,-23.554,-22.474,-22.069,-21.659,-21.104,-20.457, \\
-20.130,-19.513,-16.956,-14.613,-9.622,-6.296,-5.692,-4.774,-4.290, \\
-3.287,-2.641,-1.365,0.000(\times 72), 1.745,2.371,3.188,4.149,5.533,6.605,6.989, \\
7.874,14.684,15.973,17.128,19.544,19.913,20.422,21.317,21.594,22.789,23.500, \\
25.430,25.946,26.188,26.728,26.804,27.453,28.274,28.593,30.203,30.423\end{array}$ \\
\hline$A_{1}$ & $\begin{array}{l}-21.466,-20.837,-18.474,-18.018,-17.701,-16.184,-14.233,-13.747, \\
13.282,14.714,15.755,16.878,17.391,18.479,21.653,22.509\end{array}$ \\
\hline
\end{tabular}




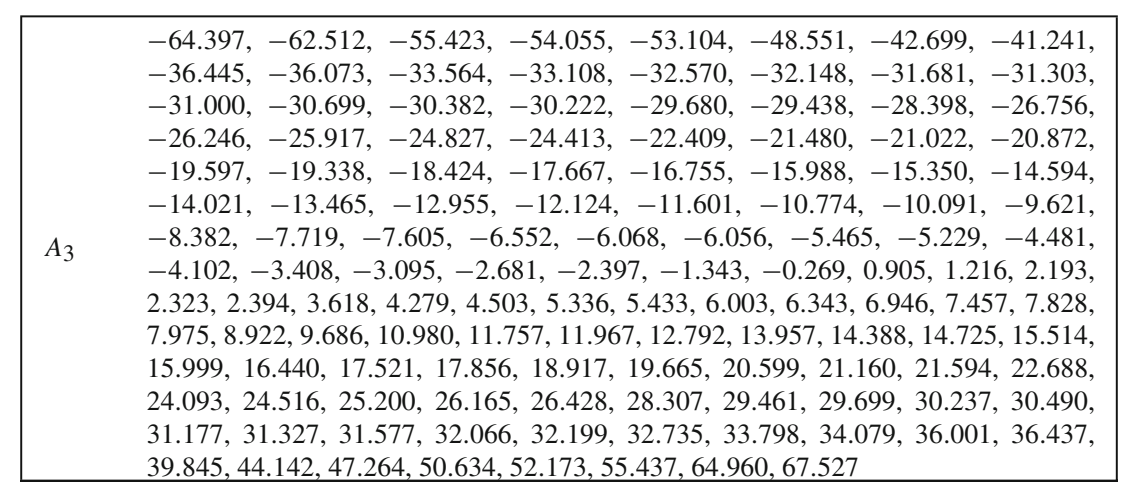

\begin{tabular}{|c|c|}
\hline \multicolumn{2}{|c|}{$R=3.408 \quad \Lambda=-243.113 \quad \operatorname{SUSY}=(0,0) \quad \operatorname{dim}(H)=0$} \\
\hline$\Psi_{128}$ & $\begin{array}{lllllll}0.224[33], & 0.056[51], & 0.038[52], & 0.062[57], & 0.906[58], & 0.219[66], & 0.071[72], \\
1.189[83], & 0.219[84], & 0.083[86], & 0.005[\dot{1} \dot{3}], & 0.079[\dot{1} \dot{5}], & 0.073[\dot{2} \dot{2}], & 0.197[\dot{2} \dot{7}], \\
0.431[\dot{3} \dot{6}], & 0.014[\dot{4} \dot{2}], & 0.031 \dot{5} \dot{1}], & 0.111 \dot{5} \dot{\dot{2}}], & 0.197[\dot{5} \dot{8}], & 0.648[\dot{6} \dot{3}], & 0.005[\dot{6} \dot{4}], \\
0.340[\dot{7} \dot{1}], 1.046[\dot{7} \dot{2}], 0.031[\dot{7} \dot{7}], 0.048[\dot{8} \dot{3}], 0.009[\dot{8} \dot{6}] ; \Delta=0.002415 & \end{array}$ \\
\hline $\mathcal{M}_{s} / g^{2}$ & $\begin{array}{l}-346.007,-278.567,-264.815,-256.957(\times 2),-247.747,-237.337,-223.700, \\
-219.163,-217.945,-210.459(\times 2),-159.981,-159.980,-156.361, \\
-145.574(\times 2),-113.734,-102.608,-101.032,-91.486(\times 2),-72.696, \\
-68.816(\times 2),-64.049,-59.755,-55.061(\times 2),-50.582,-38.079,-32.260, \\
-31.996(\times 2),-0.001(\times 3), 0.000(\times 50), 0.001(\times 3), 27.985,38.352,85.145, \\
240.058,240.059,264.953,269.001(\times 2), 280.138,355.201,369.684,371.738, \\
483.755, \quad 533.967, \quad 550.481(\times 2), 552.844,552.845,667.277,676.548(\times 2), \\
795.458(\times 2), 795.941,875.689,918.902,918.903,921.824,922.744,941.496, \\
971.258,971.260,1112.495,1176.234,1222.212,1399.693,1440.687,1481.012\end{array}$ \\
\hline $\mathcal{M}_{v} / g$ & $\begin{array}{l}-31.671,-31.106,-30.964,-29.832,-29.522,-28.773,-28.424,-28.169, \\
-27.477,-26.658,-26.068,-25.260,-24.741,-23.437,-23.226,-22.422, \\
-22.404,-19.371,-18.848,-16.417,-13.252,-9.555,-6.179,-6.049,-4.512, \\
-2.677,-1.635,-1.616,0.000(\times 72), 1.616,1.635,2.677,4.512,6.049,6.179,9.555, \\
13.252,16.417,18.848,19.371,22.404,22.422,23.226,23.437,24.741,25.260, \\
26.068,26.658,27.477,28.169,28.424,28.773,29.522,29.832,30.964,31.106, \\
31.671\end{array}$ \\
\hline$A_{1}$ & $\begin{array}{l}-23.105,-22.304,-21.113,-18.841,-17.989,-17.411,-16.542,-15.355, \\
15.355,16.542,17.411,17.989,18.841,21.113,22.304,23.105\end{array}$ \\
\hline$A_{3}$ & $\begin{array}{l}-69.314,-66.912,-63.339,-56.523,-53.968,-52.234,-49.626,-46.066, \\
-38.293,-37.815,-37.264,-37.012,-34.785,-33.953,-33.837,-33.594, \\
-33.551,-33.374,-32.415,-32.315,-31.646,-31.044,-30.936,-30.687, \\
-28.578,-27.877,-27.580,-27.059,-25.990,-25.444,-22.363,-22.097, \\
-21.603,-21.164,-21.032,-20.962,-19.752,-19.566,-19.361,-18.489, \\
-16.522,-16.460,-15.125,-13.251,-12.353,-11.686,-10.966,-10.910, \\
-10.689,-9.719,-8.172,-8.103,-7.939,-6.377,-5.847,-5.395,-4.492, \\
-3.856,-3.350,-2.722,-2.415,-1.371,-0.521,-0.297,0.297,0.521,1.371, \\
2.415,2.722,3.350,3.856,4.492,5.395,5.847,6.377,7.939,8.103,8.172,9.719, \\
10.689,10.910,10.966,11.686,12.353,13.251,15.125,16.460,16.522,18.489, \\
19.361,19.566,19.752,20.962,21.032,21.164,21.603,22.097,22.363,25.444, \\
25.990,27.059,27.580,27.877,28.578,30.687,30.936,31.044,31.646,32.315, \\
32.415,33.374,33.551,33.594,33.837,33.953,34.785,37.012,37.264,37.815, \\
38.293,46.066,49.626,52.234,53.968,56.523,63.339,66.912,69.314\end{array}$ \\
\hline
\end{tabular}




\begin{tabular}{|c|c|}
\hline \multicolumn{2}{|c|}{$R=3.419 \quad \Lambda=-247.473 \quad \operatorname{SUSY}=(0,0) \quad \operatorname{dim}(H)=0$} \\
\hline$\Psi_{128}$ & 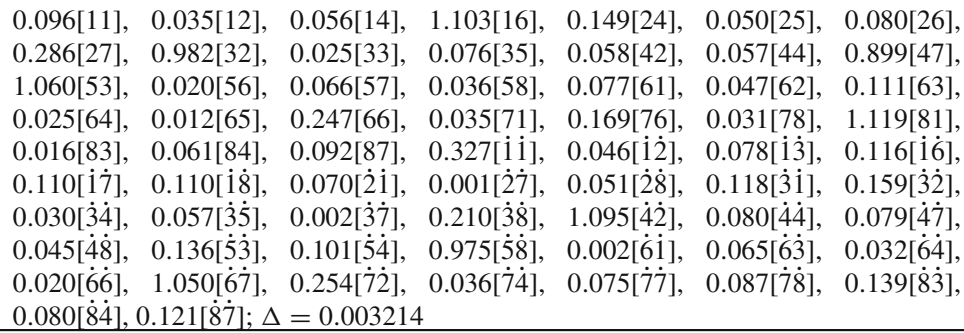 \\
\hline $\mathcal{M}_{s} / g^{2}$ & $\begin{array}{l}-348.086,-303.949,-298.976,-294.831(\times 2),-279.910,-235.417(\times 2), \\
-228.268,-215.586,-197.205(\times 2),-185.443,-167.186,-159.468, \\
-135.228(\times 2),-123.447,-106.664(\times 2),-102.297,-98.847,-94.482,-81.742, \\
-69.041,-69.040,-47.327,-43.187,-40.914,-24.760(\times 2),-0.002(\times 2), \\
-0.001(\times 7), 0.000(\times 47), 9.776(\times 2), 32.504,78.682,91.630,114.952,134.718, \\
205.746(\times 2), 223.636(\times 2), 239.487,289.571,341.145,348.421,573.681,573.683, \\
597.107,614.722,660.419,660.420,735.624,735.625,762.022,762.809,818.083, \\
821.521(\times 2), 848.103,883.470,947.722(\times 2), 1036.567,1113.057,1130.271, \\
1130.272,1133.484,1260.075,1322.483,1341.353,1410.509\end{array}$ \\
\hline $\mathcal{M}_{v} / g$ & $\begin{array}{l}-32.716,-32.646,-31.056,-30.427,-29.754,-28.914,-28.627,-27.932, \\
-27.132,-26.488,-26.242,-25.650,-24.308,-23.265,-22.257,-22.239, \\
-21.700,-19.550,-17.670,-16.381,-14.059,-12.257,-10.017,-7.254, \\
-4.994,-4.287,-2.946,-1.258,0.000(\times 72), 1.258,2.946,4.287,4.994,7.254, \\
10.017,12.257,14.059,16.381,17.670,19.550,21.700,22.239,22.257,23.265, \\
24.308,25.650,26.242,26.488,27.132,27.932,28.627,28.914,29.754,30.427, \\
31.056,32.646,32.716\end{array}$ \\
\hline$A_{1}$ & $\begin{array}{l}-23.344,-21.902,-21.265,-19.538,-18.200,-18.144,-16.792,-14.897, \\
14.897,16.792,18.144,18.200,19.538,21.265,21.902,23.344\end{array}$ \\
\hline$A_{3}$ & $\begin{array}{l}-70.031,-65.705,-63.796,-58.615,-54.600,-54.432,-50.375,-44.692, \\
-37.983,-37.846,-36.075,-35.860,-35.407,-34.764,-34.622,-34.373, \\
-34.325,-34.321,-33.094,-32.109,-31.992,-31.625,-31.345,-31.045, \\
-31.031,-29.491,-27.753,-27.355,-26.503,-25.066,-23.514,-22.710, \\
-21.498,-20.229,-19.491,-18.648,-18.540,-18.495,-18.344,-17.844, \\
-16.810,-16.778,-16.341,-15.124,-14.979,-14.381,-11.927,-11.291, \\
-9.930,-8.407,-8.385,-7.597,-7.060,-6.924,-6.662,-5.497,-5.470, \\
-4.324,-3.680,-3.612,-2.699,-0.996,-0.387,-0.302,0.302,0.387,0.996, \\
2.699,3.612,3.680,4.324,5.470,5.497,6.662,6.924,7.060,7.597,8.385,8.407, \\
9.930,11.291,11.927,14.381,14.979,15.124,16.341,16.778,16.810,17.844,18.344, \\
18.495,18.540,18.648,19.491,20.229,21.498,22.710,23.514,25.066,26.503, \\
27.355,27.753,29.491,31.031,31.045,31.345,31.625,31.992,32.109,33.094, \\
34.321,34.325,34.373,34.622,34.764,35.407,35.860,36.075,37.846,37.983, \\
44.692,50.375,54.432,54.600,58.615,63.796,65.705,70.031\end{array}$ \\
\hline
\end{tabular}

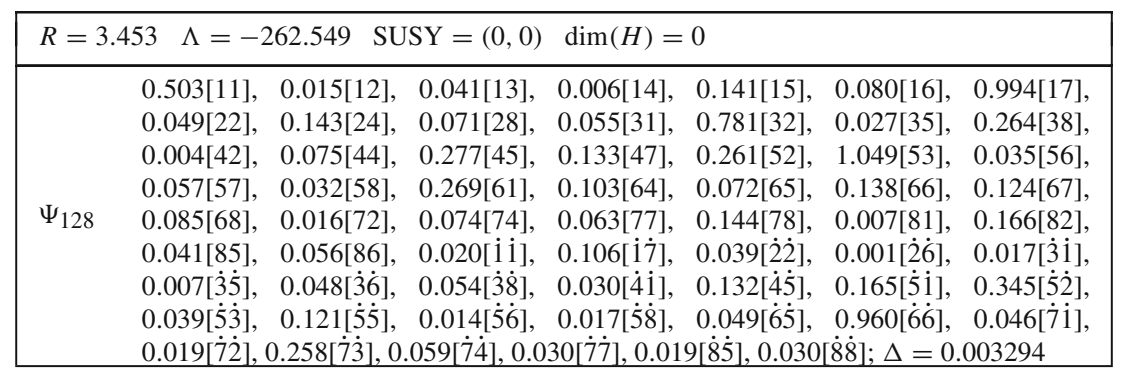




\begin{tabular}{|c|c|}
\hline $\mathcal{M}_{s} / g^{2}$ & $\begin{array}{l}-350.490,-328.284,-285.423,-280.778,-264.014,-260.375,-242.438, \\
-240.871,-226.901,-225.002,-204.076,-194.324,-191.073,-175.165, \\
-160.678,-151.082,-146.460,-142.860,-134.225,-106.643,-101.060, \\
-93.936,-93.747,-88.276,-83.435,-77.305,-74.420,-68.768,-56.360, \\
-49.847,-23.514,-14.580,-0.001(\times 4), 0.000(\times 47), 0.001(\times 5), 3.621,9.514, \\
25.403,66.901,79.117,82.339,104.198,155.774,236.279,254.064,335.049, \\
364.200,404.489,409.899,469.637,555.986,595.648,634.100,651.002,673.545, \\
747.776,772.141,783.158,871.220,888.703,938.405,979.903,996.126,1021.401, \\
1034.577,1116.021,1138.143,1157.791,1217.828,1223.772,1230.692,1275.756, \\
1292.035,1416.969,1423.936\end{array}$ \\
\hline $\mathcal{M}_{v} / g$ & $\begin{array}{l}-31.979,-31.818,-31.104,-30.813,-30.182,-30.028,-29.196,-28.792, \\
-28.731,-28.150,-27.192,-26.889,-25.528,-25.387,-23.984,-23.114, \\
-19.756,-18.673,-18.451,-16.833,-16.073,-15.661,-8.898,-7.585,-4.568, \\
-3.458,-2.697,-2.422,0.000(\times 72), 2.633,3.660,3.937,4.328,7.641,8.074, \\
13.707,15.349,15.826,18.338,18.443,19.655,21.037,23.240,23.523,23.770, \\
24.348,24.766,29.637,29.795,29.950,30.122,30.207,31.150,33.235,33.645, \\
33.738,34.208\end{array}$ \\
\hline$A_{1}$ & $\begin{array}{l}-23.586,-21.934,-21.771,-19.036,-18.752,-18.523,-17.836,-16.664, \\
14.950,15.081,17.303,17.757,19.432,24.112,24.617,24.851\end{array}$ \\
\hline$A_{3}$ & $\begin{array}{l}-70.758,-65.802,-65.312,-57.109,-56.256,-55.570,-53.507,-49.991, \\
-38.956,-38.591,-36.759,-36.417,-35.831,-35.444,-34.839,-34.741, \\
-34.589,-34.387,-34.354,-34.248,-33.609,-33.529,-31.855,-31.245, \\
-30.161,-29.851,-29.492,-27.743,-26.745,-26.587,-25.368,-23.529, \\
-22.871,-21.822,-21.594,-21.231,-20.683,-20.386,-19.373,-19.208, \\
-16.424,-15.848,-15.335,-14.964,-14.003,-13.574,-12.952,-12.155, \\
-10.061,-9.960,-7.808,-6.855,-6.665,-6.182,-5.860,-5.278,-4.836, \\
-4.646,-3.748,-3.306,-2.422,-1.834,-1.584,-0.514,0.494,0.772,1.859, \\
2.026,2.243,2.750,3.842,4.677,5.055,5.303,6.350,7.098,9.078,9.416,10.217, \\
10.796,11.615,14.646,14.934,15.127,15.603,15.671,16.343,17.186,17.706, \\
18.500,18.719,18.980,19.146,19.569,21.242,21.872,23.299,23.639,25.314, \\
25.695,25.987,27.252,27.625,31.901,32.454,32.881,33.540,33.899,34.559, \\
34.649,35.705,35.990,36.469,36.599,36.918,37.146,37.342,37.529,38.294, \\
39.334,44.849,45.243,51.908,53.270,58.295,72.335,73.852,74.554\end{array}$ \\
\hline
\end{tabular}

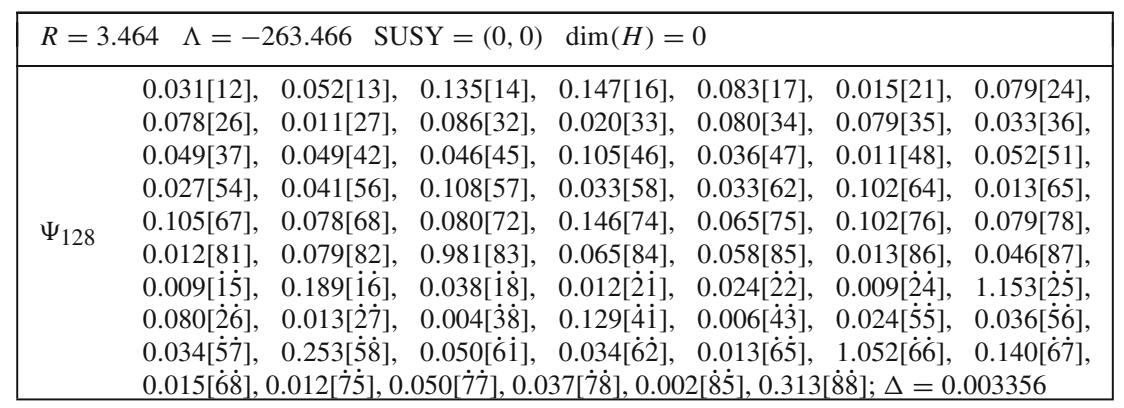




\begin{tabular}{|c|c|}
\hline $\mathcal{M}_{s} / g^{2}$ & $\begin{array}{l}-352.280,-316.182,-303.247,-289.901,-282.048,-269.525,-257.342, \\
-247.071,-245.771,-233.886,-218.051,-198.937,-194.404,-191.674, \\
-176.445,-168.172,-154.208,-142.389,-127.400,-124.937,-107.952, \\
-99.787,-96.659,-86.676,-79.255,-76.228,-70.313,-56.415,-49.956, \\
-47.372,-14.884,-0.002(\times 2),-0.001(\times 6), 0.000(\times 37), 0.001(\times 6), 0.002(\times 5), \\
24.223,24.381,58.328,117.214,140.754,179.105,202.561,269.688,307.501, \\
333.510,365.360,384.225,439.061,444.364,564.555,568.510,589.775,674.144, \\
708.903,786.850,794.066,855.095,866.030,881.137,933.922,955.482,967.940, \\
1048.350,1066.436,1154.999,1218.271,1262.999,1307.561,1388.989,1396.905, \\
1437.736,1487.872,1939.176,1943.211,1991.538,1994.034\end{array}$ \\
\hline $\mathcal{M}_{v} / g$ & $\begin{array}{l}-40.268,-40.005,-34.839,-34.255,-31.960,-30.756,-29.404,-29.046, \\
-27.683,-27.221,-26.866,-26.109,-25.004,-24.784,-23.653,-22.849, \\
-22.184,-21.965,-20.634,-19.900,-19.478,-12.016,-9.162,-6.865,-4.917, \\
-3.804,-2.981,-1.762,0.000(\times 72), 1.762,2.981,3.804,4.917,6.865,9.162, \\
12.016,19.478,19.900,20.634,21.965,22.184,22.849,23.653,24.784,25.004, \\
26.109,26.866,27.221,27.683,29.046,29.404,30.756,31.960,34.255,34.839, \\
40.005,40.268\end{array}$ \\
\hline$A_{1}$ & $\begin{array}{l}-26.502,-26.183,-22.301,-21.363,-19.473,-17.360,-16.072,-15.741, \\
15.741,16.072,17.360,19.473,21.363,22.301,26.183,26.502\end{array}$ \\
\hline$A_{3}$ & $\begin{array}{l}-79.506,-78.549,-66.903,-64.090,-58.418,-52.081,-48.217,-47.223, \\
-46.448,-46.404,-42.042,-41.710,-41.018,-40.837,-38.163,-37.911, \\
-36.472,-36.221,-35.367,-34.026,-33.788,-33.006,-32.833,-32.760, \\
-32.114,-31.277,-29.500,-29.107,-28.448,-26.950,-25.771,-24.476, \\
-23.714,-23.263,-22.832,-22.102,-21.059,-20.951,-20.050,-19.220, \\
-18.445,-17.765,-17.206,-16.307,-15.181,-14.792,-13.089,-11.668, \\
-10.845,-10.635,-9.541,-7.686,-6.726,-6.200,-5.182,-4.602,-3.571, \\
-3.173,-2.885,-2.188,-1.966,-1.681,-1.081,-0.287,0.287,1.081,1.681, \\
1.966,2.188,2.885,3.173,3.571,4.602,5.182,6.200,6.726,7.686,9.541,10.635, \\
10.845,11.668,13.089,14.792,15.181,16.307,17.206,17.765,18.445,19.220, \\
20.050,20.951,21.059,22.102,22.832,23.263,23.714,24.476,25.770,26.950, \\
28.448,29.107,29.500,31.277,32.114,32.760,32.833,33.006,33.788,34.026, \\
35.367,36.221,36.472,37.911,38.163,40.837,41.018,41.710,42.042,46.404, \\
46.448,47.223,48.217,52.081,58.418,64.090,66.903,78.549,79.506\end{array}$ \\
\hline
\end{tabular}

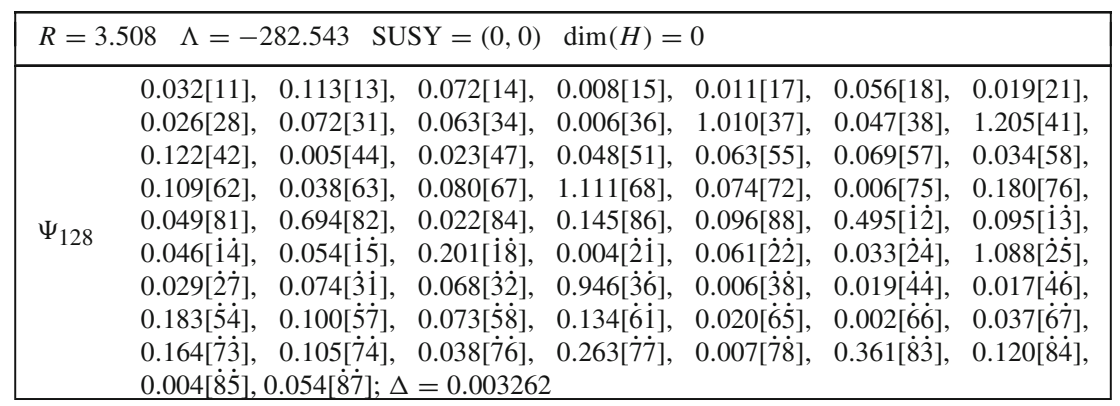




\begin{tabular}{|c|c|}
\hline $\mathcal{M}_{s} / g^{2}$ & $\begin{array}{l}-370.965,-350.665,-314.298,-268.307,-260.941,-251.442,-247.097, \\
-238.786,-235.587,-231.061,-226.052,-221.437,-221.060,-218.360, \\
-211.239,-207.882,-190.045,-179.050,-171.368,-166.818,-152.614, \\
-118.729,-109.709,-107.483,-105.410,-102.040,-96.485,-88.652,-55.579, \\
-54.085,-0.002,-0.001(\times 5), 0.000(\times 48), 0.001(\times 2), 8.372,16.432,27.502, \\
57.892,58.340,92.031,190.169,288.323,329.978,337.275,361.811,380.316, \\
385.737,387.916,424.978,494.082,580.951,647.802,688.782,758.420,777.297, \\
795.494,799.776,812.662,970.231,977.114,993.148,1040.355,1058.918, \\
1096.206,1116.103,1129.130,1147.135,1220.581,1274.199,1317.222,1329.095, \\
1388.076,1389.090,1548.215,1569.045,1672.824\end{array}$ \\
\hline $\mathcal{M}_{v} / g$ & $\begin{array}{l}-38.529,-37.432,-34.330,-34.019,-31.696,-31.052,-30.501,-28.764, \\
-27.776,-27.458,-27.294,-26.030,-25.927,-25.105,-24.306,-23.542, \\
-23.413,-20.932,-20.796,-20.492,-14.709,-11.365,-11.000,-9.570, \\
-9.285,-2.602,-2.127,-1.420,0.000(\times 72), 1.420,2.127,2.602,9.285,9.570, \\
11.000,11.365,14.709,20.492,20.796,20.932,23.413,23.542,24.306,25.105, \\
25.927,26.030,27.294,27.458,27.776,28.764,30.501,31.052,31.696,34.019, \\
34.330,37.432,38.529\end{array}$ \\
\hline$A_{1}$ & $\begin{array}{l}-26.392,-26.276,-22.501,-21.584,-21.530,-17.238,-15.462,-13.804, \\
13.804,15.462,17.237,21.530,21.584,22.501,26.276,26.392\end{array}$ \\
\hline$A_{3}$ & $\begin{array}{l}-79.175,-78.828,-67.504,-64.752,-64.590,-51.712,-46.385,-42.452, \\
-42.390,-41.413,-39.458,-39.068,-38.993,-38.694,-38.252,-37.287, \\
-36.371,-36.238,-35.138,-34.365,-33.755,-33.752,-33.290,-32.696, \\
-32.446,-32.352,-31.970,-30.152,-27.018,-26.888,-26.667,-26.242, \\
-24.653,-24.271,-23.642,-22.714,-20.984,-20.673,-19.117,-18.080, \\
-17.520,-17.219,-16.970,-16.564,-15.514,-14.379,-14.096,-13.262, \\
-11.443,-10.872,-9.616,-8.437,-6.768,-6.573,-6.429,-5.178,-4.863, \\
-4.505,-4.128,-3.937,-1.686,-1.038,-0.582,-0.258,0.258,0.582,1.038, \\
1.686,3.937,4.128,4.505,4.863,5.178,6.429,6.573,6.768,8.437,9.616,10.872, \\
11.443,13.262,14.096,14.379,15.514,16.564,16.970,17.219,17.520,18.080, \\
19.117,20.673,20.984,22.714,23.641,24.271,24.653,26.242,26.667,26.888, \\
27.018,30.152,31.970,32.352,32.446,32.696,33.290,33.752,33.755,34.365, \\
35.138,36.238,36.371,37.287,38.252,38.694,38.993,39.068,39.458,41.413, \\
42.390,42.452,46.385,51.712,64.590,64.752,67.504,78.828,79.175\end{array}$ \\
\hline
\end{tabular}

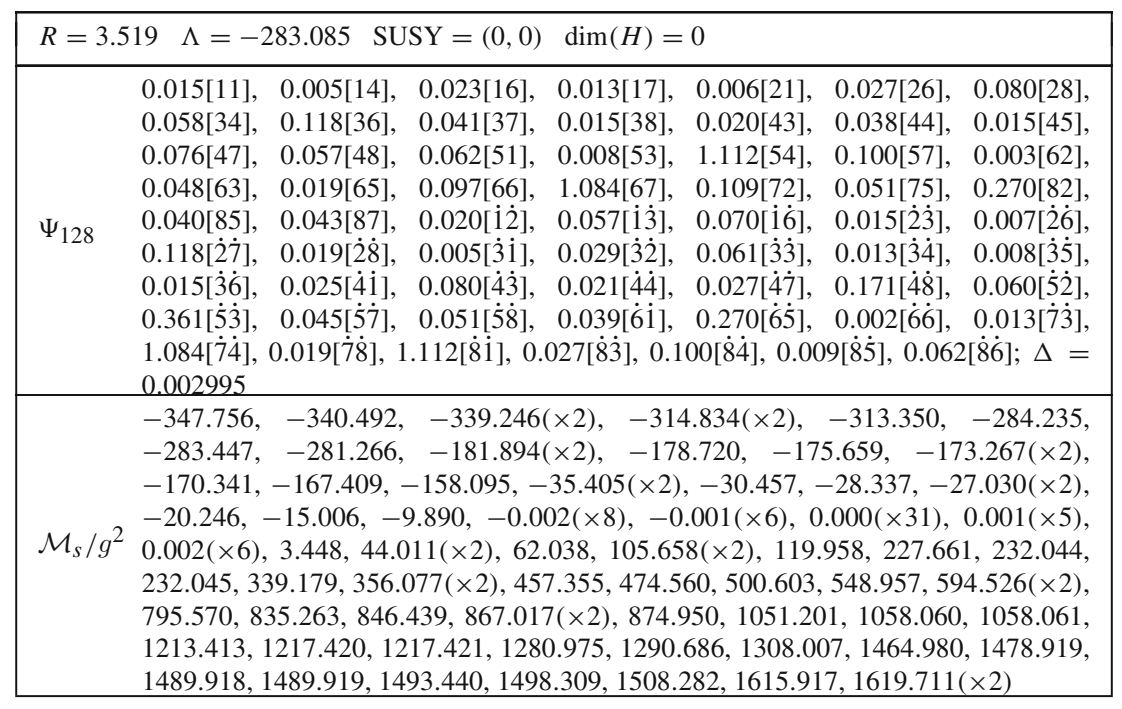




\begin{tabular}{|c|c|c|}
\hline & $-35.618,-35.579,-35.186,-34.711,-34.600,-34.254,-30.672,-30.634$, \\
& $-30.553,-30.551,-28.589,-28.507,-28.474,-25.318,-25.285,-25.270$, \\
$\mathcal{M}_{v} / g$ & $-23.577,-23.491,-22.269,-20.360,-20.341,-20.059,-7.444,-7.228,-7.162$, \\
& $-2.197,-2.182,-2.070,0.000(\times 72), 2.070,2.182,2.197,7.162,7.228,7.444$, \\
& $20.059,20.341,20.360,22.269,23.491,23.577,25.270,25.285,25.318,28.474$, \\
& $28.507,28.589,30.551,30.553,30.634,30.672,34.254,34.600,34.711,35.186$, \\
\hline \multirow{2}{*}{$A_{1}$} & $-25.579,35.618$ \\
& $-24.021,-23.871,-23.803,-21.174,-21.052,-20.901,-19.975,-19.500$, \\
& $19.500,19.975,20.901,21.052,21.174,23.803,23.871,24.021$ \\
& $-72.064,-71.613,-71.409,-63.521,-63.156,-62.704,-59.924,-58.499$, \\
& $-41.851,-41.120,-41.091,-41.018,-39.739,-39.550,-39.503,-38.601$, \\
& $-38.364,-38.242,-38.228,-38.226,-35.670,-35.620,-35.448,-35.433$, \\
& $-35.185,-35.129,-35.038,-34.904,-27.698,-27.648,-27.442,-27.227$, \\
& $-26.976,-25.091,-24.231,-24.181,-24.091,-21.491,-21.482,-21.418$, \\
& $-17.325,-17.176,-17.021,-16.445,-16.006,-14.613,-14.380,-14.299$, \\
& $-13.908,-12.766,-12.448,-12.432,-11.327,-11.189,-10.915,-2.272$, \\
& $-2.252,-2.240,-2.158,-1.848,-1.439,-0.648,-0.637,-0.457,0.457,0.637$, \\
& $0.648,1.439,1.848,2.158,2.240,2.252,2.272,10.915,11.189,11.327,12.432$, \\
& $12.448,12.766,13.908,14.299,14.380,14.613,16.006,16.445,17.021,17.176$, \\
& $17.325,21.418,21.482,21.491,24.091,24.181,24.231,25.091,26.976,27.227$, \\
& $27.442,27.648,27.698,34.904,35.038,35.129,35.185,35.433,35.448,35.620$, \\
& $35.670,38.226,38.228,38.242,38.364,38.601,39.503,39.550,39.739,41.018$, \\
& $41.091,41.120,41.851,58.499,59.924,62.704,63.156,63.521,71.409,71.613$, \\
& 72.064 & \\
\hline &
\end{tabular}

\begin{tabular}{|c|c|}
\hline \multicolumn{2}{|c|}{$R=3.525 \quad \Lambda=-286.715 \quad \operatorname{SUSY}=(0,0) \quad \operatorname{dim}(H)=0$} \\
\hline$\Psi_{128}$ & $\begin{array}{lllllll}0.005[15], & 0.161[18], & 0.082[28], & 0.119[32], & 0.341[41], & 0.766[42], & 0.080[44], \\
0.032[57], & 0.118[65], & 0.798[67], & 0.131[71], & 0.004[\dot{1} \dot{5}], & 0.372[\dot{1} \dot{7}], & 0.086[\dot{1} \dot{8}], \\
0.007[\dot{2} \dot{5}], & 0.263[\dot{2} \dot{7}], & 0.038[\dot{2} \dot{8}], & 0.788[\dot{3} \dot{1}], & 0.117[\dot{3} \dot{2}], & 0.002[\dot{3} \dot{3}], & 0.238[\dot{4} \dot{4}], \\
0.328[\dot{5} \dot{6}], & 0.060[\dot{6} \dot{6}], 0.016[\dot{7} \dot{\dot{1}}], 0.023[\dot{7} \dot{2}], 1.289[\dot{7} \dot{3}], 0.190[\dot{8} \dot{4}] ; \Delta=0.002255\end{array}$ \\
\hline $\mathcal{M}_{s} / g^{2}$ & 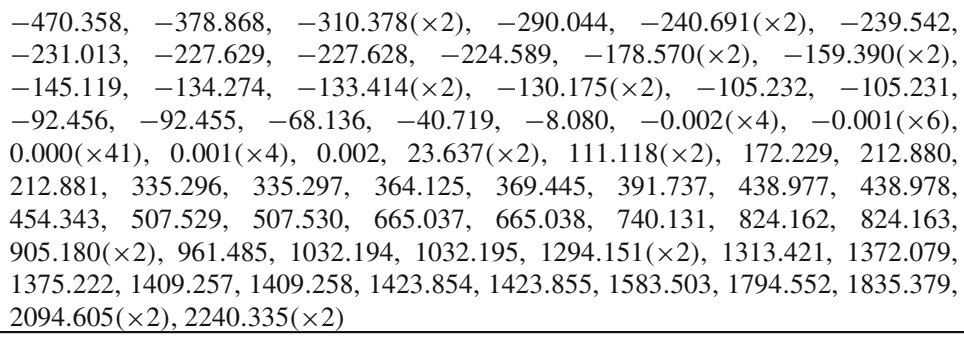 \\
\hline $\mathcal{M}_{v} / g$ & $\begin{array}{l}-42.899,-42.755,-35.713(\times 2),-33.162(\times 2),-32.127,-30.389,-29.119(\times 2), \\
-28.576(\times 2),-28.122,-25.926,-25.203(\times 2),-24.700,-23.973,-22.414(\times 2), \\
-18.257(\times 2),-6.704(\times 2),-5.488,-2.881(\times 2),-2.746,0.000(\times 72), 2.746, \\
2.881(\times 2), 5.488,6.704(\times 2), 18.257(\times 2), 22.414(\times 2), 23.973,24.700,25.203(\times 2), \\
25.926,28.122,28.576(\times 2), 29.119(\times 2), 30.389,32.127,33.162(\times 2), 35.713(\times 2), \\
42.755,42.899\end{array}$ \\
\hline$A_{1}$ & $\begin{array}{l}-27.908(\times 2),-23.174(\times 2),-19.962,-18.704(\times 2),-16.230,16.230,18.704(\times 2), \\
19.962,23.174(\times 2), 27.908(\times 2)\end{array}$ \\
\hline
\end{tabular}




\begin{tabular}{|c|c|}
\hline$A_{3}$ & $\begin{array}{l}-83.725(\times 2),-69.522(\times 2),-59.885,-56.111(\times 2),-48.691,-48.370,-48.305, \\
-46.443(\times 2),-42.768(\times 2),-39.823(\times 2),-38.527(\times 2),-37.536,-36.845, \\
-36.540,-35.414(\times 2),-35.316,-33.976,-32.821(\times 2),-30.886,-29.263(\times 2), \\
-29.104,-27.084(\times 2),-26.855,-24.106(\times 2),-23.521(\times 2),-21.621,-20.054, \\
-19.299(\times 2),-17.779(\times 2),-15.422,-12.703(\times 2),-12.291,-11.924, \\
-11.335(\times 2),-10.467(\times 2),-8.499,-7.531(\times 2),-7.294,-5.246,-3.849, \\
-2.890(\times 2),-2.649,-1.276(\times 2), 1.276(\times 2), 2.649,2.890(\times 2), 3.849,5.246, \\
7.294,7.531(\times 2), 8.499,10.467(\times 2), 11.335(\times 2), 11.924,12.291,12.703(\times 2), \\
15.422,17.779(\times 2), 19.299(\times 2), 20.054,21.621,23.521(\times 2), 24.106(\times 2), 26.855, \\
27.084(\times 2), 29.104,29.263(\times 2), 30.886,32.821(\times 2), 33.976,35.316,35.414(\times 2), \\
36.540,36.845,37.536,38.527(\times 2), 39.823(\times 2), 42.768(\times 2), 46.443(\times 2), 48.305, \\
48.370,48.691,56.111(\times 2), 59.885,69.522(\times 2), 83.725(\times 2)\end{array}$ \\
\hline
\end{tabular}

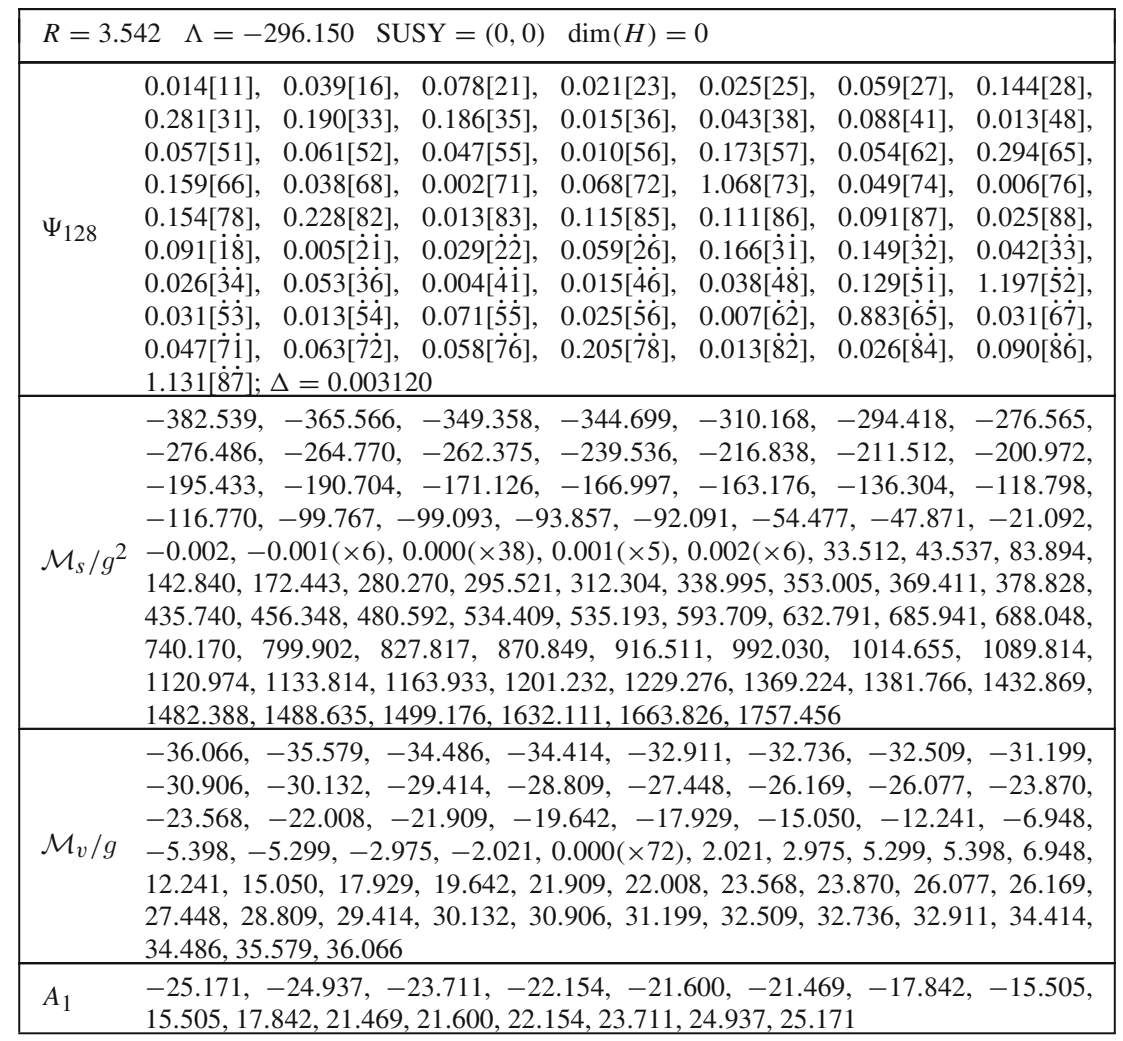




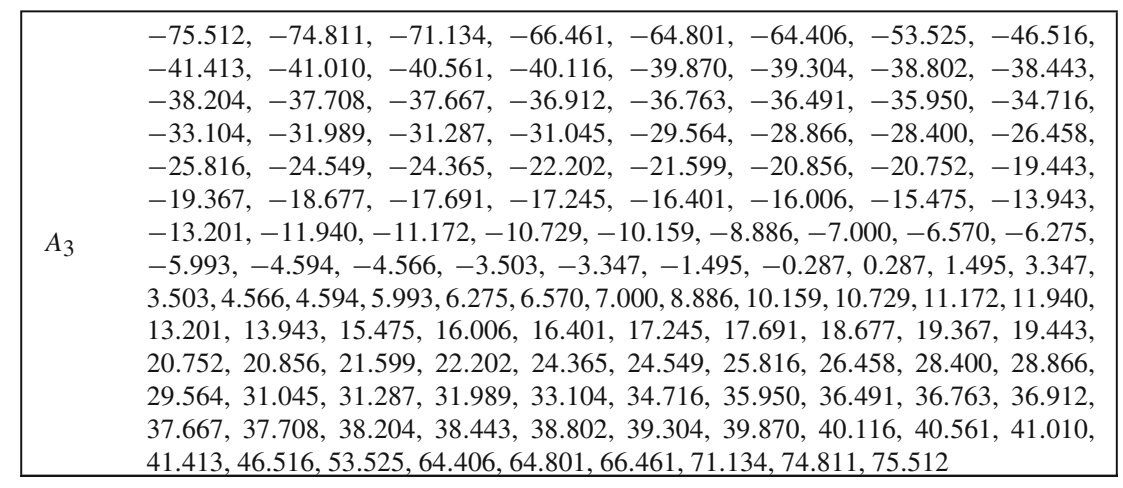

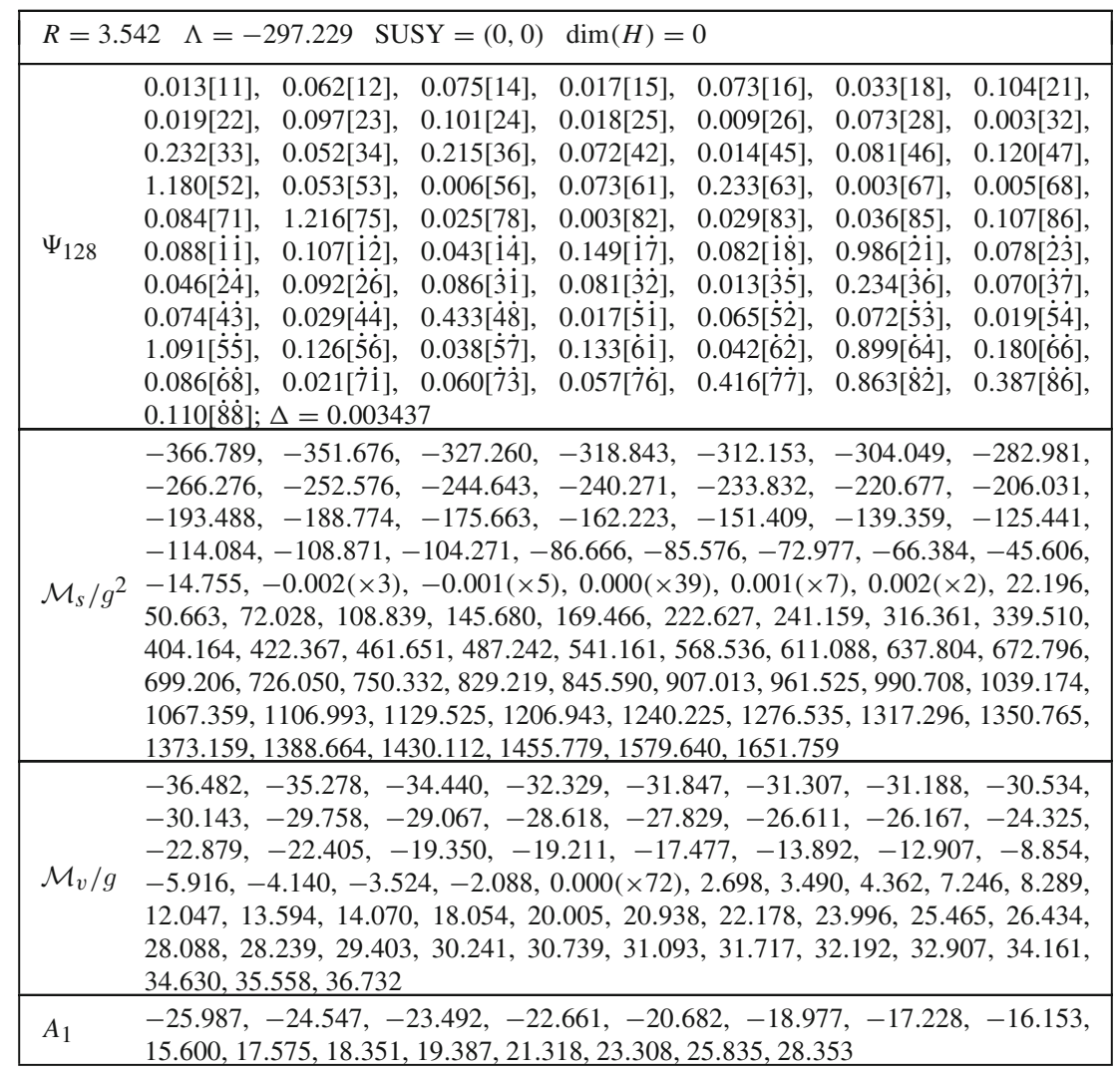




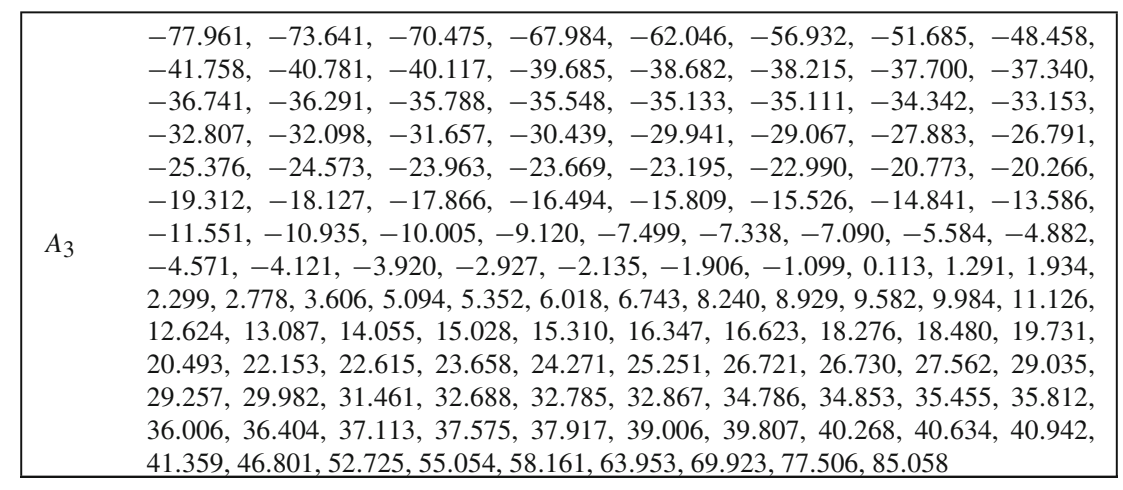

\begin{tabular}{|c|c|}
\hline \multicolumn{2}{|c|}{$R=3.545 \quad \Lambda=-297.362 \quad \operatorname{SUSY}=(0,0) \quad \operatorname{dim}(H)=0$} \\
\hline$\Psi_{128}$ & 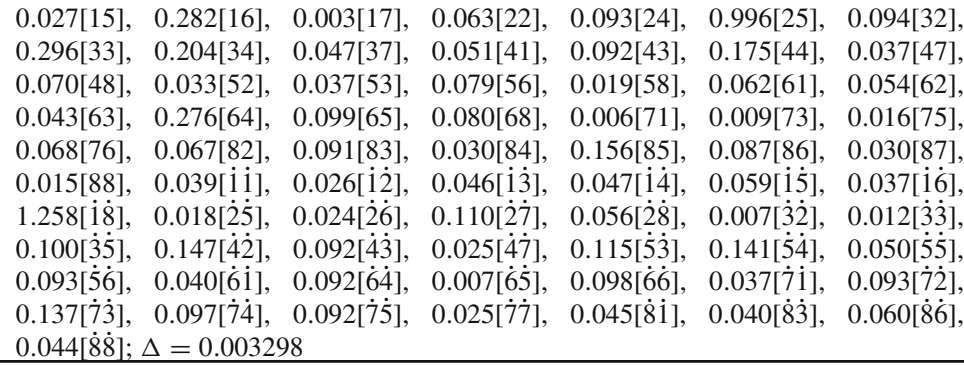 \\
\hline $\mathcal{M}_{s} / g^{2}$ & $\begin{array}{l}-424.221,-379.870,-341.645,-330.203,-317.667,-305.651,-279.363, \\
-259.385,-257.968,-248.331,-227.406,-205.376,-203.901,-186.910, \\
-180.736,-164.631,-147.942,-147.787,-121.277,-118.459,-113.986, \\
-107.801,-81.973,-79.161,-75.897,-59.486,-46.930,-31.936,-16.345, \\
-0.002(\times 5),-0.001(\times 7), 0.000(\times 42), 0.001(\times 2), 35.613,53.972,61.761,168.867, \\
203.503,311.102,335.781,356.530,402.641,410.101,410.593,448.820,490.040, \\
536.260,548.230,550.217,585.170,605.504,671.517,712.436,733.060,767.456, \\
797.309,934.515,965.403,971.008,976.028,1117.144,1146.123,1154.840, \\
1297.698,1354.639,1367.278,1374.314,1376.792,1404.086,1532.962,1658.726, \\
1684.792,1823.437,1876.943,2026.540,2066.808\end{array}$ \\
\hline $\mathcal{M}_{v} / g$ & $\begin{array}{l}-39.020,-38.747,-36.708,-35.849,-33.998,-32.797,-32.715,-31.880, \\
-30.629,-29.390,-28.878,-28.776,-27.214,-27.143,-27.118,-24.777, \\
-24.235,-23.399,-22.950,-22.196,-20.993,-16.076,-8.267,-7.685,-5.569, \\
-5.474,-2.704,-1.893,0.000(\times 72), 1.893,2.704,5.474,5.569,7.685,8.267, \\
16.076,20.993,22.196,22.950,23.399,24.235,24.777,27.118,27.143,27.214, \\
28.776,28.878,29.390,30.629,31.880,32.715,32.797,33.998,35.849,36.708, \\
38.747,39.020\end{array}$ \\
\hline$A_{1}$ & $\begin{array}{l}-26.181,-25.107,-24.936,-23.578,-22.491,-20.115,-17.414,-17.168, \\
17.168,17.414,20.115,22.491,23.578,24.936,25.107,26.181\end{array}$ \\
\hline
\end{tabular}




\begin{tabular}{|c|c|}
\hline$A_{3}$ & $\begin{array}{l}-78.543,-75.320,-74.807,-70.733,-67.472,-60.344,-52.243,-51.503, \\
-46.070,-45.911,-42.439,-42.043,-41.589,-41.406,-40.012,-39.402, \\
-38.637,-38.446,-38.243,-37.843,-37.351,-37.130,-36.112,-35.582, \\
-34.939,-33.758,-32.602,-31.870,-29.922,-28.684,-28.599,-27.719, \\
-25.892,-25.466,-25.188,-23.494,-23.329,-21.577,-21.529,-20.877, \\
-20.818,-19.022,-17.759,-17.541,-16.545,-16.008,-14.700,-13.649, \\
-13.103,-12.615,-11.524,-11.095,-9.027,-9.010,-8.691,-8.145,-7.819, \\
-7.231,-6.844,-5.659,-4.729,-4.648,-2.918,-1.779,1.779,2.918,4.648, \\
4.729,5.659,6.844,7.231,7.819,8.145,8.691,9.010,9.027,11.095,11.524,12.615, \\
13.103,13.649,14.700,16.008,16.545,17.541,17.759,19.022,20.818,20.877, \\
21.529,21.577,23.329,23.494,25.188,25.466,25.892,27.719,28.599,28.684, \\
29.922,31.870,32.602,33.758,34.939,35.582,36.112,37.130,37.351,37.843, \\
38.243,38.446,38.637,39.402,40.012,41.406,41.589,42.043,42.439,45.911, \\
46.070,51.503,52.243,60.344,67.472,70.733,74.807,75.320,78.543\end{array}$ \\
\hline
\end{tabular}

\begin{tabular}{|c|c|}
\hline \multicolumn{2}{|c|}{$R=3.555 \quad \Lambda=-298.931 \quad \operatorname{SUSY}=(0,0) \quad \operatorname{dim}(H)=0$} \\
\hline$\Psi_{128}$ & 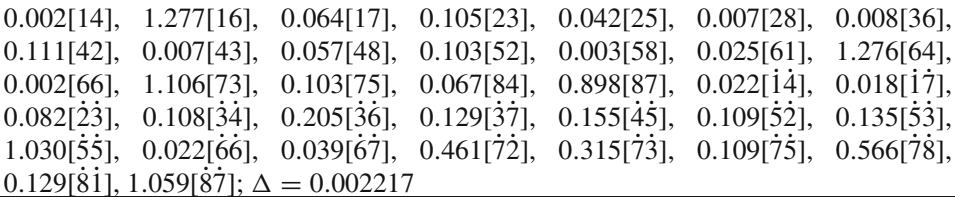 \\
\hline $\mathcal{M}_{s} / g^{2}$ & $\begin{array}{l}-447.037,-332.267(\times 3),-331.176(\times 2),-321.515(\times 3),-298.638(\times 3), \\
-239.282,-164.204,-156.037(\times 3),-68.037(\times 2),-59.288,-53.954(\times 3), \\
-40.418(\times 3),-27.846(\times 2),-27.845,-0.002,-0.001(\times 5), 0.000(\times 40), \\
0.001(\times 6), 0.002(\times 4), 38.254,173.112(\times 2), 326.149(\times 2), 326.150,339.062, \\
339.063(\times 2), 381.021,407.809(\times 2), 407.810,449.724,449.725,533.518,533.519, \\
533.520,708.954,749.693,749.694,1016.129,1016.130,1016.131,1028.192, \\
1028.193,1028.194,1065.610,1065.611,1065.613,1284.346,1284.347,1305.234, \\
1305.235,1359.187,1538.612,1538.614,1538.615,1774.741,1774.745(\times 2), \\
1798.306,1798.308\end{array}$ \\
\hline $\mathcal{M}_{v} / g$ & $\begin{array}{l}-35.773(\times 3),-33.227(\times 3),-32.865(\times 3),-31.196,-29.349(\times 2),-29.348, \\
-27.879(\times 3),-26.927(\times 2),-18.569,-18.443(\times 3),-8.283(\times 3),-2.724(\times 3), \\
0.000(\times 72), 2.724(\times 3), 8.283(\times 3), 18.443(\times 3), 18.569,26.927(\times 2), 27.879(\times 3), \\
29.348,29.349(\times 2), 31.196,32.865(\times 3), 33.227(\times 3), 35.773(\times 3)\end{array}$ \\
\hline$A_{1}$ & $-23.702(\times 3),-23.017(\times 3),-19.136(\times 2), 19.136(\times 2), 23.017(\times 3), 23.702(\times 3)$ \\
\hline$A_{3}$ & $\begin{array}{l}-71.106(\times 3),-69.052(\times 3),-57.409(\times 2),-41.540(\times 3),-41.480(\times 3),-40.838, \\
-40.726,-38.293(\times 3),-37.367(\times 3),-37.339,-35.964(\times 2),-34.527(\times 3), \\
-31.035,-29.281(\times 3),-27.676(\times 2),-21.606(\times 3),-20.459(\times 3),-19.218(\times 3), \\
-16.755(\times 3),-15.137(\times 2),-13.800(\times 3),-11.221,-9.877,-9.017(\times 3), \\
-6.822(\times 2),-6.439(\times 3),-3.325(\times 3), 3.325(\times 3), 6.439(\times 3), 6.822(\times 2), \\
9.017(\times 3), 9.877,11.221,13.800(\times 3), 15.137(\times 2), 16.755(\times 3), 19.218(\times 3), \\
20.459(\times 3), 21.606(\times 3), 27.676(\times 2), 29.281(\times 3), 31.035,34.527(\times 3), 35.964(\times 2), \\
37.339, \quad 37.367(\times 3), 38.293(\times 3), 40.726,40.838,41.480(\times 3), 41.540(\times 3), \\
57.409(\times 2), 69.052(\times 3), 71.106(\times 3)\end{array}$ \\
\hline
\end{tabular}

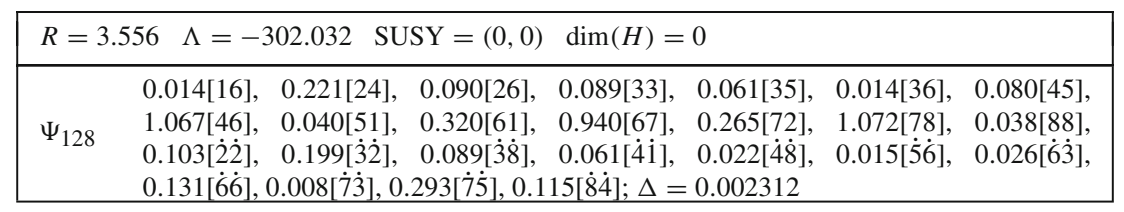




\begin{tabular}{|c|c|}
\hline $\mathcal{M}_{s} / g^{2}$ & $\begin{array}{l}-386.341,-382.394,-341.265,-313.215,-308.090,-303.074,-293.317, \\
-271.204,-262.874,-242.644,-240.900,-206.720,-204.910,-204.468, \\
-183.884,-174.629,-171.944,-142.779,-135.216,-119.659,-115.685, \\
-104.608,-92.407,-86.749,-73.062,-60.763,-57.835,-54.245,-21.744, \\
-1.503,-0.001(\times 3), 0.000(\times 43), 0.001(\times 7), 0.002(\times 3), 22.527,72.345,85.758, \\
110.349,140.951,206.910,277.084,306.366,324.156,371.136,452.393,500.118, \\
551.226,568.579,590.304,644.636,654.482,712.003,754.583,759.933,852.183, \\
906.378,994.030,1039.181,1060.731,1094.061,1144.517,1144.848,1200.778, \\
1235.434,1263.633,1328.175,1369.608,1386.272,1445.430,1475.982,1554.262, \\
1659.933,1684.011,1727.822,2163.517,2181.807\end{array}$ \\
\hline $\mathcal{M}_{v} / g$ & $\begin{array}{l}-40.271,-39.998,-35.381,-35.338,-32.982,-32.703,-32.617,-31.522, \\
-30.817,-30.569,-29.189,-28.505,-28.170,-27.853,-26.047,-25.634, \\
-24.436,-23.462,-21.958,-19.698,-17.707,-17.047,-14.314,-6.091, \\
-5.864,-4.342,-3.582,-0.872,0.000(\times 72), 1.449,3.964,4.346,5.233,6.967, \\
10.676,16.059,18.353,20.767,21.490,23.648,24.084,24.879,26.849,27.339, \\
27.714,28.759,29.017,30.277,31.208,32.739,32.789,34.260,34.587,34.982, \\
37.491,38.239,38.799\end{array}$ \\
\hline$A_{1}$ & $\begin{array}{l}-26.767,-25.999,-23.890,-22.768,-22.029,-21.015,-18.700,-15.927, \\
14.979,18.613,21.742,22.750,22.892,23.805,24.127,28.186\end{array}$ \\
\hline$A_{3}$ & $\begin{array}{l}-80.301,-77.998,-71.669,-68.304,-66.087,-63.044,-56.101,-47.780, \\
-46.036,-45.770,-44.539,-43.978,-41.529,-40.428,-40.393,-39.780, \\
-38.797,-38.678,-38.589,-37.539,-36.357,-36.254,-34.948,-34.912, \\
-34.432,-32.374,-32.197,-30.573,-29.117,-28.494,-28.236,-27.454, \\
-27.131,-26.334,-25.526,-24.416,-24.367,-22.474,-22.089,-21.326, \\
-19.979,-19.902,-19.314,-17.627,-17.050,-16.108,-15.365,-15.083, \\
-13.877,-12.193,-11.415,-10.392,-9.917,-7.393,-5.930,-5.890,-4.717, \\
-3.849,-3.628,-3.167,-2.194,-1.682,-1.016,-0.658,-0.010,1.903,2.140, \\
2.390,2.614,3.216,4.400,4.997,5.234,5.566,7.901,8.782,10.215,10.642,12.247, \\
13.965,14.026,15.186,16.718,17.849,18.377,19.151,19.292,19.529,20.267, \\
21.941,23.461,23.467,23.596,25.040,26.429,27.612,27.623,28.606,28.608, \\
30.276,30.881,31.634,33.482,34.534,34.948,36.039,36.323,37.363,38.118, \\
38.275,39.111,39.276,39.821,39.959,41.005,41.054,43.277,43.316,44.937, \\
45.752,45.989,55.839,65.227,68.251,68.675,71.415,72.380,84.558\end{array}$ \\
\hline
\end{tabular}

\begin{tabular}{|c|c|}
\hline \multicolumn{2}{|c|}{$R=3.567 \quad \Lambda=-305.970 \quad \operatorname{SUSY}=(0,0) \quad \operatorname{dim}(H)=0$} \\
\hline$\Psi_{128}$ & 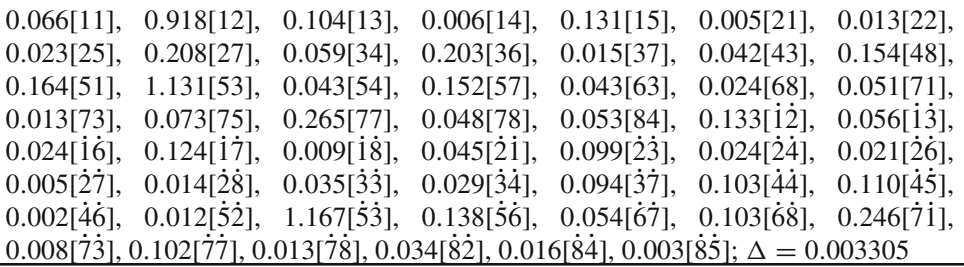 \\
\hline $\mathcal{M}_{s} / g^{2}$ & $\begin{array}{l}-416.977,-362.637,-353.642,-337.594,-336.378,-323.428,-308.851, \\
-283.767,-273.873,-256.960,-252.667,-245.515,-237.865,-199.498, \\
-182.496,-151.731,-147.908,-137.874,-133.932,-114.529,-114.362, \\
-109.935,-109.756,-84.294,-78.837,-56.881,-47.190,-26.569,-0.002(\times 3), \\
-0.001(\times 5), 0.000(\times 35), 0.001(\times 8), 0.002(\times 5), 35.355,45.385,128.018,159.348, \\
166.986,197.968,250.511,275.876,356.149,391.955,421.383,435.139,444.073, \\
489.352,575.280,580.981,610.968,649.202,689.845,705.675,722.443,802.980, \\
806.490,939.969,961.694,975.317,983.606,1100.164,1179.330,1200.596, \\
1212.260,1251.396,1411.209,1432.049,1442.051,1476.550,1516.249,1639.328, \\
1681.227,1689.569,1719.366,1803.869,1905.238,1952.417\end{array}$ \\
\hline
\end{tabular}




\begin{tabular}{|c|c|}
\hline & $-38.332,-37.453,-37.193,-36.562,-35.317,-33.440,-33.438,-32.289$, \\
& $-31.356,-30.517,-30.424,-28.908,-28.030,-27.140,-26.285,-25.905$, \\
& $-25.001,-23.902,-22.496,-21.857,-21.547,-15.570,-13.418,-6.380$, \\
$\mathcal{M}_{v} / g$ & $-5.467,-4.844,-3.857,-2.087,0.000(\times 72), 2.087,3.857,4.844,5.467,6.380$, \\
& $13.418,15.570,21.547,21.857,22.496,23.902,25.001,25.905,26.285,27.140$, \\
& $28.030,28.908,30.425,30.517,31.356,32.289,33.438,33.440,35.317,36.562$, \\
& $37.193,37.453,38.332$ \\
& $-26.321,-25.472,-24.013,-23.873,-22.336,-21.692,-19.466,-16.688$, \\
\hline$A_{1}$ & $16.688,19.466,21.692,22.336,23.873,24.013,25.472,26.321$ \\
& $-78.964,-76.416,-72.039,-71.620,-67.008,-65.075,-58.397,-50.064$, \\
& $-44.252,-44.108,-43.392,-43.181,-41.385,-41.004,-40.573,-40.503$, \\
& $-39.851,-39.464,-39.045,-38.511,-38.354,-37.844,-36.844,-35.932$, \\
& $-35.778,-34.442,-32.947,-32.603,-30.706,-30.329,-29.915,-29.086$, \\
& $-25.877,-25.274,-24.022,-23.993,-22.935,-22.878,-22.647,-21.780$, \\
& $-21.360,-20.271,-18.109,-17.808,-17.490,-16.978,-15.545,-14.792$, \\
& $-13.105,-12.223,-12.193,-11.303,-9.839,-9.249,-7.966,-7.303,-6.752$, \\
& $-5.871,-5.639,-4.651,-4.603,-4.180,-2.570,-1.922,1.922,2.570,4.180$, \\
& $4.603,4.651,5.639,5.871,6.752,7.303,7.966,9.249,9.839,11.303,12.193,12.223$, \\
& $13.105,14.792,15.545,16.978,17.490,17.808,18.109,20.271,21.360,21.780$, \\
& $22.647,22.878,22.935,23.993,24.022,25.274,25.877,29.086,29.915,30.329$, \\
& $30.706,32.603,32.947,34.442,35.778,35.932,36.844,37.844,38.354,38.512$, \\
& $39.045,39.464,39.851,40.503,40.573,41.004,41.385,43.181,43.392,44.108$, \\
& $44.252,50.064,58.397,65.075,67.008,71.620,72.039,76.416,78.964$
\end{tabular}

\begin{tabular}{|c|c|}
\hline \multicolumn{2}{|c|}{$R=3.575 \quad \Lambda=-311.092 \quad \operatorname{SUSY}=(0,0) \quad \operatorname{dim}(H)=0$} \\
\hline$\Psi_{128}$ & $\begin{array}{lllllll}0.170[11], & 0.027[12], & 0.056[13], & 0.142[14], & 1.021[15], & 0.023[17], & 0.101[21], \\
0.229[23], & 0.047[26], & 0.229[27], & 0.027[31], & 0.329[43], & 0.027[45], & 0.116[47], \\
0.053[48], & 0.027[51], & 0.325[54], & 0.082[56], & 0.053[58], & 0.101[63], & 0.012[72], \\
0.017[73], & 1.092[78], & 0.023[83], & 0.142[84], & 0.056[87], & 0.043[88], & 0.022[\dot{1} \dot{1}], \\
1.163[\dot{1} \dot{2}], & 0.117[\dot{1} \dot{3}], & 0.005[\dot{1} \dot{4}], & 0.025[\dot{1} \dot{5}], & 0.047[\dot{1} \dot{8}], & 0.002[\dot{2} \dot{8}], & 0.013[\dot{3} \dot{2}], \\
0.013[\dot{3} \dot{6}], & 0.019[\dot{3} \dot{8}], & 0.025[\dot{4} \dot{3}], & 0.184[\dot{4} \dot{8}], & 0.030[\dot{5} \dot{1}], & 0.025[\dot{5} \dot{3}], & 0.184[\dot{5} \dot{4}], \\
0.025[\dot{5} \dot{6}], & 0.010[\dot{5} \dot{7}], & 0.003[\dot{6} \dot{2}], & 0.086[\dot{6} \dot{4}], & 0.067[\dot{6} \dot{5}], & 0.086[\dot{6} \dot{8}], & 0.069[\dot{7} \dot{2}], \\
0.136[\dot{7} \dot{3}], & 0.022[\dot{8} \dot{1}], 1.163[\dot{8} \dot{6}] ; \Delta=0.003014 & & & \\
\end{array}$ \\
\hline $\mathcal{M}_{s} / g^{2}$ & $\begin{array}{l}-386.207,-380.234,-337.486,-322.464,-320.202,-305.140,-300.074, \\
-289.454,-264.502,-256.935,-249.532,-242.635,-235.857,-227.238, \\
-210.111,-188.769,-185.877,-179.857,-164.922,-139.586,-118.923, \\
-96.772,-87.164,-85.832,-73.245,-65.608,-35.095,-22.933,-10.316, \\
-0.002(\times 3),-0.001(\times 6), 0.000(\times 36), 0.001(\times 4), 0.002(\times 7), 31.576,53.685, \\
74.574,104.426,230.292,236.606,259.217,339.889,342.643,377.088,420.592, \\
442.254,520.533,575.339,616.889,627.614,685.082,693.354,713.538,744.717, \\
758.637,829.997,862.354,903.018,994.062,1078.598,1088.086,1151.688, \\
1194.366,1216.798,1267.615,1308.525,1321.055,1370.016,1400.464,1417.772, \\
1419.322,1433.747,1468.384,1525.153,1640.035,2050.637,2063.792\end{array}$ \\
\hline $\mathcal{M}_{v} / g$ & $\begin{array}{l}-38.888,-37.842,-36.790,-35.409,-34.863,-33.437,-32.619,-31.672, \\
-30.979,-30.883,-30.425,-29.114,-28.095,-27.346,-26.969,-26.522, \\
-24.973,-23.348,-20.940,-20.149,-17.906,-14.885,-14.419,-8.824, \\
-6.047,-5.247,-3.763,-1.914,0.000(\times 72), 2.137,2.965,5.262,6.136,9.128, \\
14.757,15.165,17.047,20.887,21.454,24.438,25.118,25.288,26.295,28.146, \\
28.606,28.908,30.245,30.298,30.825,31.162,33.060,33.334,33.786,35.811, \\
36.607,38.229,39.174\end{array}$ \\
\hline$A_{1}$ & $\begin{array}{l}-28.223,-26.588,-24.952,-22.140,-21.170,-19.923,-17.567,-17.448, \\
16.415,18.506,19.755,21.966,22.555,24.769,25.559,28.487\end{array}$ \\
\hline
\end{tabular}




\begin{tabular}{|c|c|}
\hline$A_{3}$ & $\begin{array}{l}-84.670,-79.765,-74.856,-66.421,-63.510,-59.770,-52.700,-52.345, \\
-44.922,-43.959,-43.427,-42.943,-40.753,-40.499,-39.507,-38.991, \\
-38.721,-38.310,-38.273,-37.075,-37.003,-36.402,-35.744,-35.491, \\
-35.292,-33.785,-33.349,-31.707,-31.561,-30.200,-29.837,-29.030, \\
-28.945,-26.914,-25.150,-23.640,-23.096,-22.514,-22.278,-21.343, \\
-20.229,-19.412,-18.887,-16.943,-16.792,-15.892,-15.199,-14.729, \\
-13.613,-12.735,-12.248,-9.836,-9.648,-8.715,-7.933,-7.681,-7.154, \\
-6.343,-5.294,-5.102,-3.311,-2.311,-2.146,-0.747,0.837,1.995,3.006, \\
3.117,4.469,5.401,6.855,7.327,7.771,7.803,9.480,9.615,10.990,11.276,11.892, \\
13.541,13.549,15.686,15.701,16.919,17.782,18.668,19.794,20.628,21.194, \\
21.507,22.177,23.235,24.086,24.453,27.265,28.575,28.775,30.440,30.584, \\
31.719,32.155,32.746,33.154,35.567,35.602,36.378,36.466,36.924,37.084, \\
37.543,38.216,38.409,38.958,39.920,40.026,40.778,42.694,43.197,44.491, \\
45142.4924555 .519592646589767666 .74307 .676\end{array}$ \\
\hline
\end{tabular}

\begin{tabular}{|c|c|}
\hline \multicolumn{2}{|c|}{$R=3.593 \quad \Lambda=-318.537 \quad \operatorname{SUSY}=(0,0) \quad \operatorname{dim}(H)=0$} \\
\hline$\Psi_{128}$ & 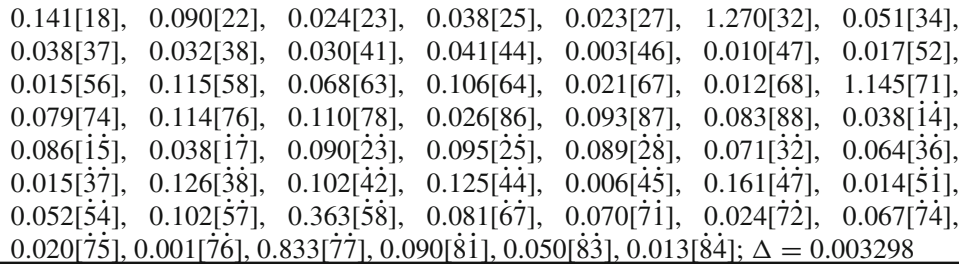 \\
\hline $\mathcal{M}_{s} / g^{2}$ & $\begin{array}{l}-419.126,-386.789,-375.199,-345.874,-330.482,-325.349,-288.305, \\
-274.369,-269.598,-253.236,-241.638,-240.083,-220.341,-196.904, \\
-193.652,-186.234,-182.037,-150.239,-131.112,-128.564,-122.830, \\
-118.522,-102.769,-92.827,-87.114,-84.176,-83.545,-52.094,-21.762, \\
-0.734,-0.718,-0.658,-0.565,-0.550,-0.541,-0.509,-0.468(\times 2),-0.436, \\
-0.413,-0.375,-0.350,-0.349,-0.302(\times 2),-0.257,-0.235,-0.181,-0.178, \\
-0.174,-0.165,-0.127,-0.109,-0.094,-0.067,-0.035,-0.016,0.003,0.006, \\
0.040,0.086,0.092,0.104,0.115,0.122,0.126,0.156,0.199,0.216,0.239,0.270, \\
0.306,0.310,0.328,0.362,0.399,0.454,0.481,0.493,0.521,0.546,0.560,0.622, \\
0.659,0.681,0.887,39.118,61.265,129.148,161.448,206.379,244.428,304.575, \\
369.406,413.356,419.280,485.831,529.016,545.198,582.814,585.355,691.384, \\
802.910,819.539,827.872,909.731,932.273,934.332,955.068,1048.965,1063.493, \\
1097.430,1136.604,1147.730,1327.559,1368.622,1376.590,1407.272,1469.230, \\
1506.607,1522.489,1541.723,1568.161,1614.129,1863.587,1888.001,2203.249, \\
2217.957\end{array}$ \\
\hline $\mathcal{M}_{v} / g$ & $\begin{array}{l}-41.611,-41.108,-36.749,-35.992,-35.292,-34.094,-33.501,-32.632, \\
-32.295,-30.946,-30.619,-29.452,-28.734,-27.595,-27.352,-25.297, \\
-24.145,-23.257,-22.330,-21.587,-18.348,-15.669,-12.934,-9.530, \\
-7.308,-4.368,-3.913,-2.587,0.000(\times 72), 2.587,3.913,4.368,7.308,9.530, \\
12.934,15.669,18.348,21.587,22.330,23.257,24.145,25.297,27.352,27.595, \\
28.734,29.452,30.619,30.946,32.295,32.632,33.501,34.094,35.293,35.992, \\
36.749,41.108,41.611\end{array}$ \\
\hline$A_{1}$ & $\begin{array}{l}-29.637,-27.554,-24.909,-23.511,-20.755,-19.962,-17.889,-17.023, \\
17.023,17.889,19.962,20.755,23.511,24.909,27.554,29.637\end{array}$ \\
\hline
\end{tabular}




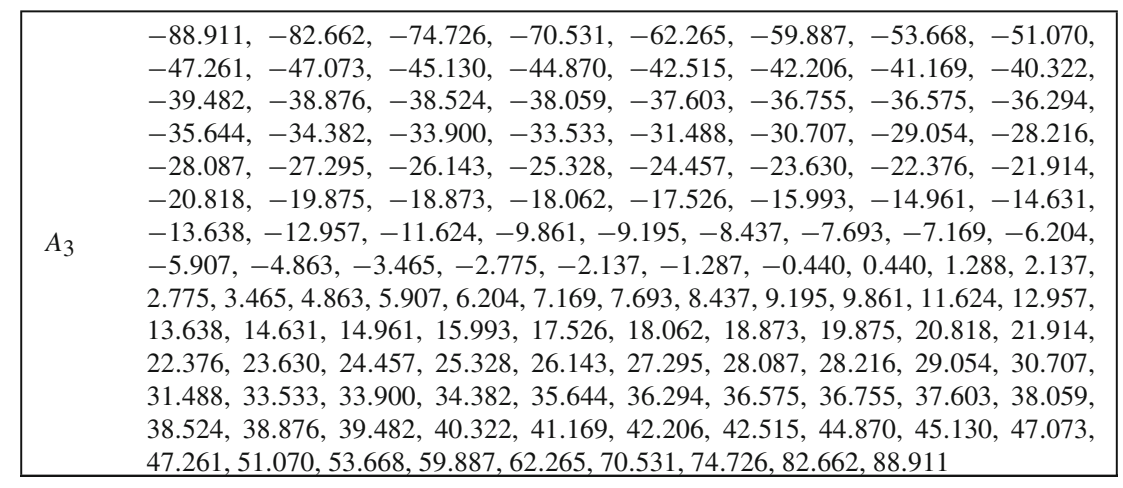

\begin{tabular}{|c|c|}
\hline \multicolumn{2}{|c|}{$R=3.599 \quad \Lambda=-321.202 \quad \operatorname{SUSY}=(0,0) \quad \operatorname{dim}(H)=0$} \\
\hline$\Psi_{128}$ & 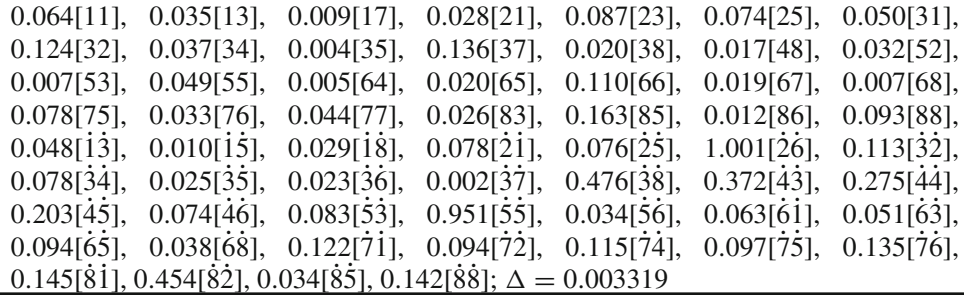 \\
\hline $\mathcal{M}_{s} / g^{2}$ & $\begin{array}{l}-415.123,-385.705,-374.406,-353.787,-344.822,-330.699,-306.077, \\
-289.904,-284.961,-275.848,-249.464,-230.665,-216.182,-212.373, \\
-206.805,-197.096,-175.151,-157.882,-146.764,-139.922,-120.754, \\
-109.431,-104.623,-78.511,-60.006,-47.544,-35.062,-26.850,-0.002, \\
-0.001(\times 4), 0.000(\times 38), 0.001(\times 7), 0.002(\times 6), 28.567,39.389,97.440,118.201, \\
195.146,203.583,210.896,251.198,315.547,329.943,347.403,423.450,468.391, \\
520.379,527.641,634.987,651.785,657.536,715.215,720.087,787.544,858.921, \\
862.315,907.699,1013.620,1040.305,1055.128,1112.886,1133.009,1140.459, \\
1285.721,1288.123,1324.914,1371.717,1407.316,1411.178,1447.463,1489.215, \\
1500.769,1568.490,1617.088,1685.495,1723.497,1821.322\end{array}$ \\
\hline $\mathcal{M}_{v} / g$ & $\begin{array}{l}-38.658,-37.443,-36.902,-35.456,-35.141,-33.989,-32.492,-31.419, \\
-31.273,-31.171,-30.402,-30.370,-29.648,-28.608,-27.455,-25.110, \\
-24.276,-23.113,-22.325,-21.585,-19.400,-15.191,-14.350,-11.441, \\
-7.354,-5.394,-3.316,-2.335,0.000(\times 72), 2.335,3.316,5.394,7.354,11.441, \\
14.350,15.191,19.400,21.585,22.325,23.113,24.276,25.110,27.455,28.608, \\
29.648,30.370,30.402,31.171,31.273,31.419,32.492,33.989,35.141,35.456, \\
36.902,37.443,38.658\end{array}$ \\
\hline$A_{1}$ & $\begin{array}{l}-27.273,-26.502,-25.069,-22.661,-22.289,-21.907,-18.752,-15.990, \\
15.990,18.752,21.907,22.289,22.661,25.069,26.502,27.273\end{array}$ \\
\hline
\end{tabular}




\begin{tabular}{|c|c|}
\hline$A_{3}$ & $\begin{array}{l}-81.819,-79.506,-75.206,-67.982,-66.868,-65.721,-56.255,-47.969, \\
-43.438,-43.151,-42.239,-41.588,-41.408,-40.733,-40.146,-39.602, \\
-39.184,-38.550,-38.386,-38.095,-37.939,-37.530,-36.818,-36.488, \\
-36.116,-34.929,-32.930,-32.290,-31.980,-30.820,-30.606,-29.670, \\
-27.605,-27.362,-26.631,-26.226,-22.993,-22.445,-21.396,-21.029, \\
-20.739,-20.041,-18.877,-18.600,-16.808,-16.707,-16.220,-15.369, \\
-14.113,-12.994,-11.681,-10.090,-9.805,-9.515,-8.815,-7.709,-7.634, \\
-6.444,-5.451,-4.770,-4.226,-2.424,-1.160,-0.396,0.396,1.160,2.424, \\
4.226,4.770,5.451,6.444,7.634,7.709,8.815,9.515,9.805,10.090,11.681,12.994, \\
14.113,15.369,16.220,16.707,16.808,18.600,18.877,20.041,20.739,21.029, \\
21.396,22.445,22.993,26.226,26.631,27.362,27.605,29.670,30.606,30.820, \\
31.980,32.290,32.930,34.929,36.117,36.488,36.818,37.530,37.939,38.095, \\
38.386,38.550,39.184,39.602,40.146,40.733,41.408,41.588,42.239,43.151, \\
43.438,47.969,56.255,65.721,66.868,67.982,75.206,79.506,81.819\end{array}$ \\
\hline
\end{tabular}

\begin{tabular}{|c|c|}
\hline \multicolumn{2}{|c|}{$R=3.611 \quad \Lambda=-325.675 \quad \operatorname{SUSY}=(0,0) \quad \operatorname{dim}(H)=0$} \\
\hline$\Psi_{128}$ & 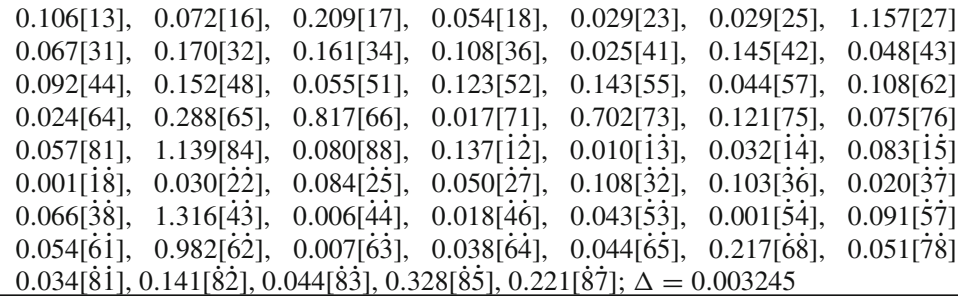 \\
\hline $\mathcal{M}_{s} / g^{2}$ & $\begin{array}{l}-447.354,-420.934,-409.211,-349.413,-330.036,-329.964,-287.745 \\
-284.939,-281.448,-252.887,-250.939,-246.680,-227.628,-203.721 \\
-194.825,-168.730,-156.232,-135.177,-124.781,-119.686,-117.083 \\
-111.297,-103.036,-85.372,-71.623,-55.461,-31.163,-23.960,-5.984 \\
-0.003,-0.002(\times 9),-0.001(\times 9), 0.000(\times 33), 0.001(\times 4), 20.231,145.971 \\
156.946,188.968,210.115,232.521,287.340,348.518,393.443,446.466,473.528 \\
503.039,534.690,572.265,631.900,654.424,703.357,792.955,799.786,863.861 \\
886.285,955.568,1002.195,1075.930,1082.496,1265.620,1299.799,1323.857 \\
1422.707,1443.013,1492.641,1506.613,1529.664,1589.265,1677.492,1735.844 \\
1802.840,1856.604,1897.996,2312.078,2322.875,2783.385,2788.254\end{array}$ \\
\hline $\mathcal{M}_{v} / g$ & $\begin{array}{l}-44.970,-44.756,-39.735,-39.577,-36.475,-36.367,-34.388,-33.202 \\
-31.880,-31.678,-30.723,-30.046,-29.626,-28.745,-26.883,-26.331 \\
-25.969,-24.984,-24.235,-22.072,-20.673,-17.032,-9.614,-7.779,-6.885 \\
-4.645,-3.571,-2.476,0.000(\times 72), 2.476,3.571,4.645,6.885,7.779,9.614 \\
17.032,20.673,22.072,24.235,24.984,25.969,26.331,26.883,28.745,29.626 \\
30.046,30.723,31.678,31.880,33.202,34.388,36.367,36.475,39.577,39.735 \\
44.756,44.970\end{array}$ \\
\hline$A_{1}$ & $\begin{array}{l}-31.607,-29.726,-25.517,-23.524,-20.982,-19.744,-18.941,-17.877 \\
17.877,18.941,19.744,20.982,23.524,25.517,29.726,31.607\end{array}$ \\
\hline
\end{tabular}




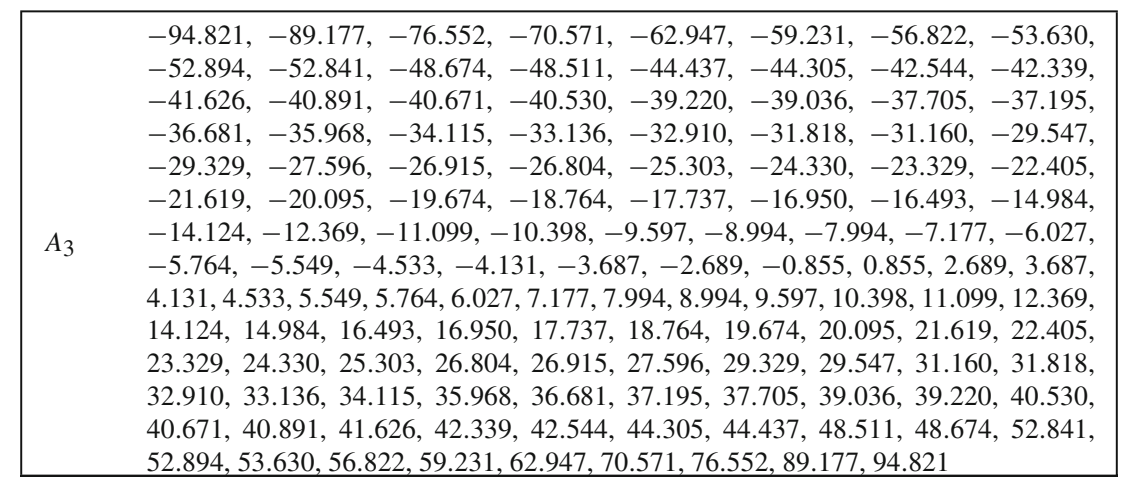

\begin{tabular}{|c|c|}
\hline \multicolumn{2}{|c|}{$R=3.653 \quad \Lambda=-345.473 \quad \operatorname{SUSY}=(0,0) \quad \operatorname{dim}(H)=0$} \\
\hline$\Psi_{128}$ & 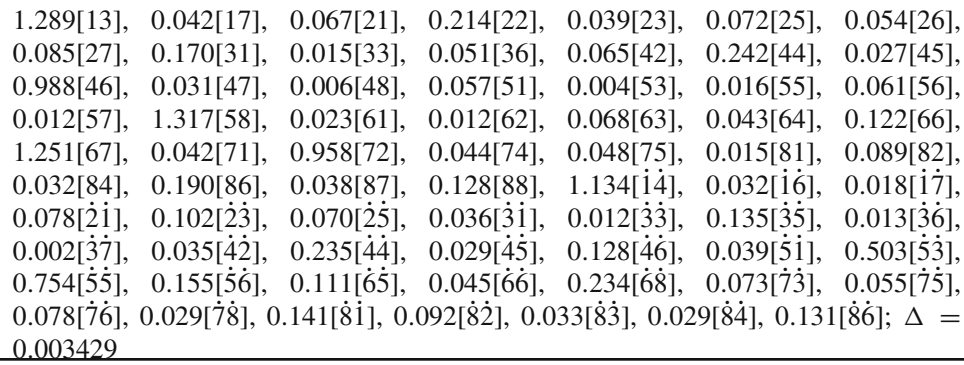 \\
\hline $\mathcal{M}_{s} / g^{2}$ & $\begin{array}{l}-438.750,-425.160,-389.984,-371.729,-361.650,-344.454,-316.607, \\
-294.070,-283.177,-272.548,-263.057,-254.359,-235.755,-223.704, \\
-180.180,-169.716,-159.476,-147.802,-132.866,-114.734,-102.474, \\
-97.108,-85.664,-81.052,-62.645,-52.887,-33.338,-0.002(\times 6), \\
-0.001(\times 7), 0.000(\times 33), 0.001(\times 5), 0.002(\times 5), 33.221,44.954,92.030,131.157, \\
140.480,188.506,269.227,298.044,351.495,354.033,442.365,488.264,544.591, \\
602.287,655.136,675.733,710.628,715.748,802.373,886.252,936.150,995.955, \\
1021.965,1063.128,1085.586,1187.070,1213.334,1272.902,1355.155,1360.815, \\
1449.185,1468.293,1482.001,1533.157,1593.841,1632.143,1663.023,1739.102, \\
1747.012,1813.350,1884.982,2044.819,2066.363,2245.182,2250.469\end{array}$ \\
\hline $\mathcal{M}_{v} / g$ & $\begin{array}{l}-43.081,-42.035,-38.851,-37.815,-35.955,-35.717,-35.057,-34.494, \\
-33.348,-32.724,-31.967,-31.305,-30.812,-30.572,-29.153,-27.406, \\
-27.165,-25.774,-25.016,-22.994,-22.222,-20.719,-12.483,-12.353, \\
-8.081,-5.206,-4.724,-2.262,0.000(\times 72), 2.261,3.503,4.901,10.151,10.623, \\
15.429,19.173,21.589,22.498,23.989,24.650,25.832,27.013,28.312,28.862, \\
30.718,31.512,32.755,33.536,34.070,34.853,35.325,36.093,37.988,38.752, \\
39.488,42.182,43.229\end{array}$ \\
\hline$A_{1}$ & $\begin{array}{l}-29.953,-27.398,-27.087,-23.778,-23.452,-22.090,-19.642,-19.586, \\
16.986,20.342,21.588,23.042,25.198,26.347,28.980,30.501\end{array}$ \\
\hline
\end{tabular}




\begin{tabular}{|c|c|}
\hline$A_{3}$ & $\begin{array}{l}-89.859,-82.193,-81.260,-71.334,-70.355,-66.269,-58.926,-58.758, \\
-49.329,-48.989,-45.882,-45.414,-44.818,-43.984,-43.442,-42.777, \\
-42.021,-41.635,-40.881,-40.120,-39.497,-39.054,-38.517,-38.319, \\
-37.494,-37.194,-35.746,-35.002,-34.806,-33.703,-32.476,-32.027, \\
-31.222,-30.923,-28.612,-27.649,-25.802,-25.306,-25.074,-24.393, \\
-23.479,-23.023,-21.986,-19.804,-18.962,-18.541,-18.041,-16.661, \\
-15.796,-14.762,-13.524,-11.362,-9.394,-8.901,-8.203,-8.079,-7.168, \\
-5.994,-5.301,-4.310,-3.597,-1.878,-1.611,0.800,1.144,1.929,2.289,2.986, \\
3.264,3.980,5.798,6.744,8.104,8.898,10.427,10.791,11.569,13.384,14.257, \\
15.694,16.624,17.763,18.057,19.158,20.264,21.303,22.177,22.597,24.074, \\
24.907,25.546,25.775,27.132,27.967,28.293,29.153,30.688,32.371,33.253, \\
34.113,35.403,36.983,37.500,37.678,38.324,39.009,39.777,40.735,41.009, \\
41.280,41.874,42.662,43.167,44.349,45.544,45.780,45.902,46.169,47.853, \\
48.210,50.958,61.027,64.764,69.127,75.593,79.041,86.940,91.504\end{array}$ \\
\hline
\end{tabular}

\begin{tabular}{|c|c|}
\hline \multicolumn{2}{|c|}{$R=3.666 \quad \Lambda=-353.424 \quad \operatorname{SUSY}=(0,0) \quad \operatorname{dim}(H)=0$} \\
\hline$\Psi_{128}$ & 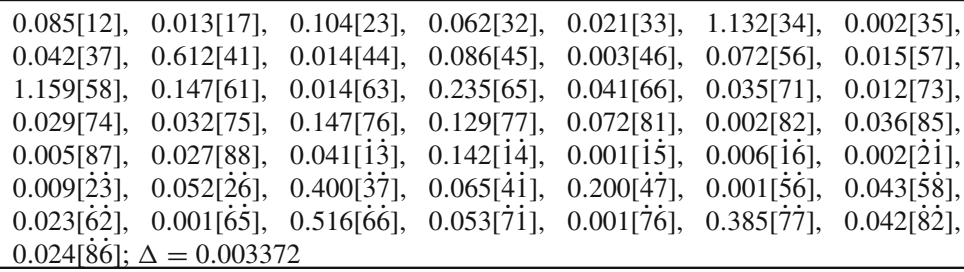 \\
\hline $\mathcal{M}_{s} / g^{2}$ & $\begin{array}{l}-412.689,-404.118,-360.077,-354.833,-353.060,-344.022,-336.283, \\
-327.206,-307.942,-278.494,-276.604,-264.722,-218.195,-207.439, \\
-198.433,-174.623,-161.892,-157.846,-136.211,-105.275,-104.482, \\
-96.657,-86.533,-76.214,-74.882,-60.463,-41.670,-20.596,-5.032, \\
-0.003(\times 2),-0.002(\times 8),-0.001(\times 6), 0.000(\times 21), 0.001(\times 4), 0.002(\times 7), \\
0.003(\times 6), 0.004(\times 2), 61.427,81.378,88.158,140.946,225.940,416.861,465.391, \\
526.871,538.838,638.801,686.805,744.871,755.483,825.455,869.653,910.587, \\
924.447,942.117,1013.437,1050.530,1095.184,1109.888,1145.760,1173.055, \\
1303.668,1379.816,1407.333,1411.290,1486.982,1531.536,1579.090,1641.056, \\
1656.181,1797.281,1828.530,1977.992,1987.184,2256.302,2263.098,2779.893, \\
2780.728,3715.845,3719.505\end{array}$ \\
\hline $\mathcal{M}_{v} / g$ & $\begin{array}{l}-47.268,-46.770,-41.612,-41.023,-39.544,-39.319,-36.610,-35.440, \\
-34.912,-34.821,-34.129,-33.798,-33.234,-33.171,-31.093,-29.977, \\
-26.240,-26.099,-24.221,-22.296,-21.596,-15.209,-14.595,-11.348, \\
-7.610,-3.774,-3.281,-2.458,0.000(\times 72), 1.569,3.406,3.788,8.960,12.235, \\
14.528,15.180,21.401,23.958,25.665,27.141,27.964,28.693,29.856,31.132, \\
31.254,32.125,32.284,32.877,35.865,36.028,36.736,37.886,37.903,41.460, \\
41.511,50.008,50.034\end{array}$ \\
\hline$A_{1}$ & $\begin{array}{l}-30.907,-29.491,-27.363,-25.832,-25.788,-24.227,-21.113,-17.371, \\
17.664,20.864,21.720,23.821,28.239,29.061,29.235,31.489\end{array}$ \\
\hline
\end{tabular}




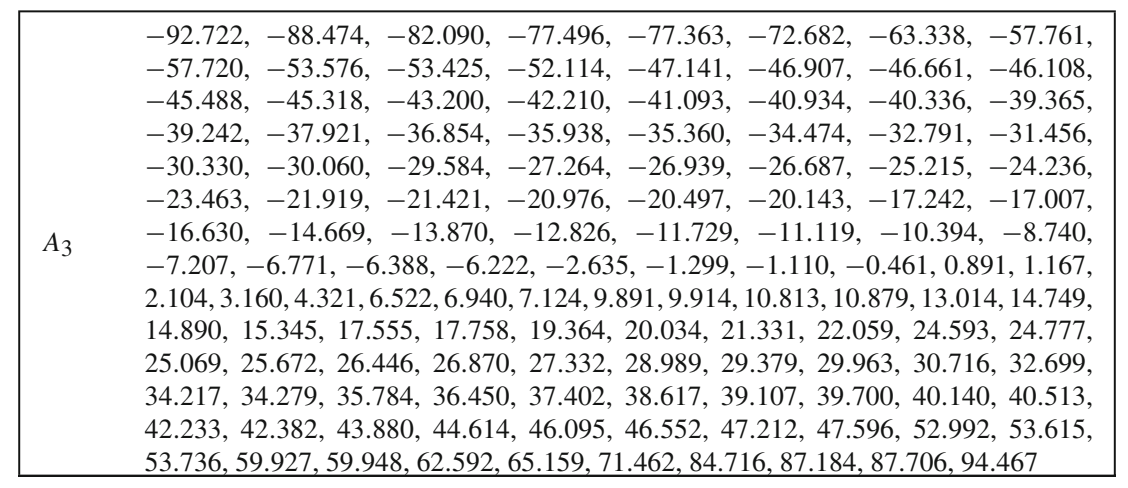

\begin{tabular}{|c|c|}
\hline \multicolumn{2}{|c|}{$R=3.667 \quad \Lambda=-352.079 \quad \operatorname{SUSY}=(0,0) \quad \operatorname{dim}(H)=0$} \\
\hline$\Psi_{128}$ & 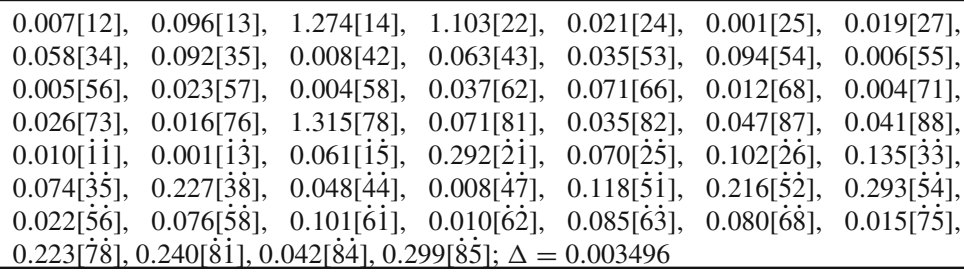 \\
\hline $\mathcal{M}_{s} / g^{2}$ & $\begin{array}{l}-518.093,-475.240,-404.398,-398.042,-397.928,-383.477,-360.978, \\
-302.898,-296.873,-289.689,-276.729,-241.352,-197.824,-196.770, \\
-182.572,-181.030,-152.041,-147.831,-142.116,-126.692,-120.208, \\
-85.735,-70.359,-52.935,-36.690,-8.487,-4.163,-0.003(\times 4),-0.002(\times 10), \\
-0.001(\times 5), 0.000(\times 27), 0.001(\times 6), 0.002(\times 4), 48.289,117.988,170.024,179.167, \\
226.382,271.077,286.786,319.898,372.947,380.313,381.497,480.276,572.017, \\
672.710,747.323,751.484,772.360,805.846,846.129,869.025,921.858,990.356, \\
1010.082,1078.362,1138.905,1186.431,1326.246,1348.050,1354.947,1429.995, \\
1484.663,1515.641,1559.531,1613.732,1638.362,1691.437,1733.222,1747.647, \\
1788.362,1891.260,1898.241,1973.484,2049.375,2172.058,2208.026\end{array}$ \\
\hline $\mathcal{M}_{v} / g$ & $\begin{array}{l}-45.309,-44.592,-38.523,-37.544,-37.044,-36.079,-36.030,-34.710, \\
-33.314,-33.161,-31.150,-30.598,-29.824,-29.694,-28.948,-28.173, \\
-27.386,-27.123,-25.042,-25.014,-20.763,-20.480,-17.337,-11.016, \\
-10.963,-4.728,-4.692,-0.595,0.000(\times 72), 0.595,4.692,4.728,10.963,11.016, \\
17.337,20.480,20.763,25.014,25.042,27.123,27.386,28.173,28.948,29.694, \\
29.824,30.598,31.150,33.161,33.314,34.710,36.030,36.079,37.044,37.544, \\
38.523,44.592,45.309\end{array}$ \\
\hline$A_{1}$ & $\begin{array}{l}-32.083,-28.049,-27.828,-25.625,-22.016,-20.735,-19.267,-19.053, \\
19.053,19.267,20.735,22.016,25.625,27.828,28.049,32.083\end{array}$ \\
\hline
\end{tabular}




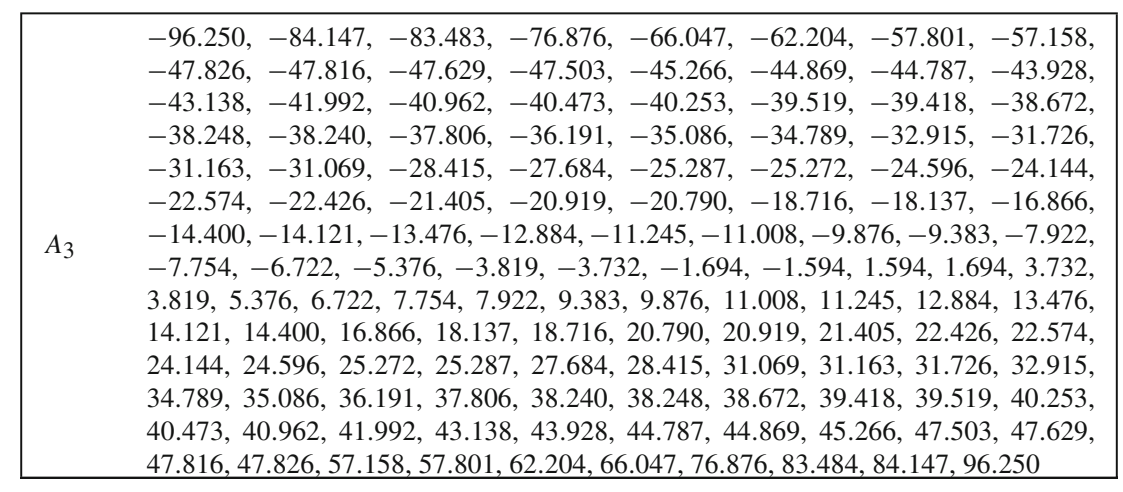

\begin{tabular}{|c|c|}
\hline \multicolumn{2}{|c|}{$R=3.704 \quad \Lambda=-371.297 \quad \operatorname{SUSY}=(0,0) \quad \operatorname{dim}(H)=0$} \\
\hline$\Psi_{128}$ & 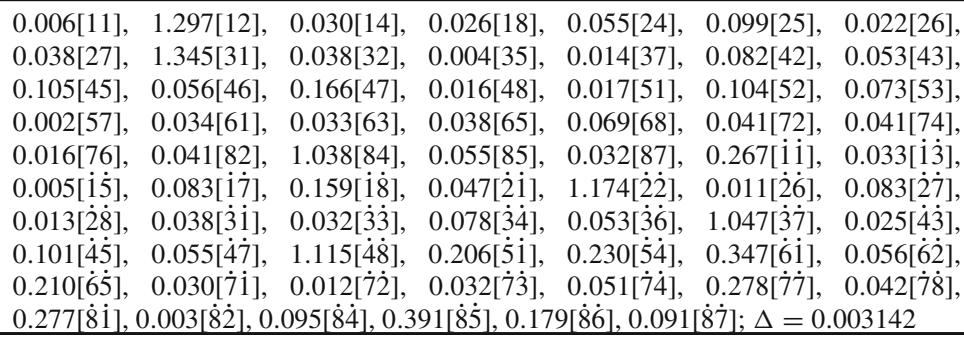 \\
\hline $\mathcal{M}_{s} / g^{2}$ & $\begin{array}{l}-518.645,-488.219,-441.179,-421.766,-372.373,-347.144,-338.428, \\
-310.045,-293.800,-280.299,-255.195,-249.484,-229.546,-218.229, \\
-202.357,-166.887,-158.190,-135.943,-127.223,-118.571,-102.825, \\
-95.663,-80.870,-66.230,-60.858,-46.448,-0.003(\times 5),-0.002(\times 8), \\
-0.001(\times 5), 0.000(\times 25), 0.001(\times 5), 0.002(\times 7), 0.003,15.690,50.407,91.543, \\
145.885,200.325,211.480,276.379,286.183,360.357,368.309,450.637,515.639, \\
567.491,639.370,703.341,743.777,806.807,853.048,904.573,973.049,1035.274, \\
1038.133,1082.802,1164.090,1181.261,1249.651,1309.938,1381.563,1399.454, \\
1438.282,1526.707,1565.780,1640.739,1648.593,1711.217,1718.853,1738.598, \\
1763.125,1890.330,1957.796,2020.765,2105.378,2245.741,2249.676,2396.200, \\
2432.677\end{array}$ \\
\hline $\mathcal{M}_{v} / g$ & $\begin{array}{l}-44.618,-44.079,-41.992,-39.484,-38.273,-36.949,-36.372,-36.268, \\
-35.583,-34.996,-32.892,-32.753,-31.222,-30.805,-30.018,-29.243, \\
-29.028,-28.063,-26.299,-25.596,-23.934,-20.596,-18.949,-9.891, \\
-7.611,-5.925,-5.218,-1.561,0.000(\times 72), 1.991,5.551,5.785,8.257,10.015, \\
19.178,19.941,22.489,23.857,26.695,26.995,28.616,29.012,29.521,31.892, \\
32.212,33.077,33.703,35.012,35.591,35.738,36.201,36.330,39.551,41.293, \\
42.016,43.669,44.026\end{array}$ \\
\hline$A_{1}$ & $\begin{array}{l}-31.462,-29.722,-26.778,-25.133,-24.669,-23.243,-22.502,-19.083, \\
19.518,20.363,22.033,25.795,27.328,28.049,28.410,31.097\end{array}$ \\
\hline
\end{tabular}




\begin{tabular}{|c|c|}
\hline$A_{3}$ & $\begin{array}{l}-94.385,-89.167,-80.335,-75.399,-74.007,-69.728,-67.507,-57.250, \\
-50.349,-50.185,-47.963,-46.896,-46.605,-46.161,-45.834,-45.429, \\
-44.363,-43.990,-43.770,-42.698,-42.100,-41.414,-40.791,-40.163, \\
-39.959,-39.262,-38.842,-38.494,-37.722,-35.270,-34.738,-34.234, \\
-32.283,-31.241,-29.653,-29.079,-28.296,-26.747,-25.661,-24.314, \\
-23.141,-22.924,-22.437,-21.827,-20.822,-19.926,-19.063,-18.228, \\
-16.059,-15.666,-14.699,-13.418,-11.508,-9.846,-8.770,-8.092,-7.752, \\
-6.866,-6.470,-5.245,-4.549,-2.271,-1.904,-1.821,0.318,1.293,2.332, \\
3.877,5.409,5.538,6.633,6.944,7.663,8.351,9.538,12.482,13.885,15.732,16.712, \\
17.542,18.756,19.356,20.712,20.958,22.265,22.730,23.531,24.336,24.653, \\
25.544,26.461,26.875,29.470,30.368,31.570,32.089,32.519,33.413,34.987, \\
35.672,37.884,38.677,39.138,39.490,40.255,40.936,41.571,42.096,42.807, \\
43.272,44.720,45.138,45.640,46.080,46.484,46.903,47.623,47.917,50.161, \\
50.504,58.554,61.088,66.098,77.386,81.983,84.147,85.230,93.290\end{array}$ \\
\hline
\end{tabular}

\begin{tabular}{|c|c|}
\hline \multicolumn{2}{|c|}{$R=3.770 \quad \Lambda=-411.138 \quad \operatorname{SUSY}=(0,0) \quad \operatorname{dim}(H)=0$} \\
\hline$\Psi_{128}$ & 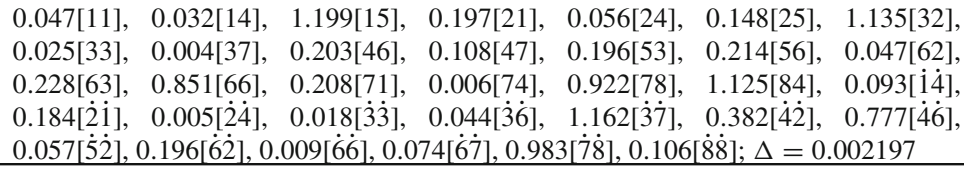 \\
\hline $\mathcal{M}_{s} / g^{2}$ & $\begin{array}{l}-536.177,-463.001,-436.783,-410.551,-401.184,-389.318,-352.169, \\
-350.777,-343.795,-323.539,-296.234,-272.370,-271.078,-266.261, \\
-248.836,-244.110,-239.322,-233.693,-220.994,-197.842,-174.751, \\
-152.707,-143.595,-127.619,-108.697,-70.573,-54.212,-39.066,-24.138, \\
-2.755,-0.002(\times 6),-0.001(\times 6), 0.000(\times 32), 0.001(\times 9), 0.002(\times 3), 20.542, \\
188.109,282.916,398.278,416.790,526.924,587.360,676.595,836.382,865.947, \\
873.193,904.594,1010.269,1026.780,1040.272,1073.627,1195.954,1221.618, \\
1285.541,1306.478,1439.706,1449.180,1547.326,1549.015,1556.801,1609.815, \\
1651.638,1695.482,1712.824,1780.189,1801.508,1966.553,2376.168,2391.866, \\
2412.219,2431.539,2435.926,2444.884,2936.841,2998.228,3354.367,3382.563\end{array}$ \\
\hline $\mathcal{M}_{v} / g$ & $\begin{array}{l}-52.707,-52.569,-45.547,-44.253,-43.905,-43.834,-39.194,-38.124, \\
-37.984,-37.463,-36.981,-34.868,-34.545,-34.066,-31.362,-30.953, \\
-28.858,-24.699,-24.528,-19.308,-17.782,-16.128,-15.002,-12.429, \\
-9.112,-7.473,-4.368,-3.989,0.000(\times 72), 3.989,4.368,7.473,9.112,12.429, \\
15.002,16.128,17.782,19.308,24.528,24.699,28.858,30.953,31.362,34.066, \\
34.545,34.868,36.981,37.463,37.984,38.124,39.194,43.834,43.905,44.253, \\
45.547,52.569,52.707\end{array}$ \\
\hline$A_{1}$ & $\begin{array}{l}-39.643,-38.986,-26.498,-24.828,-22.043,-21.314,-20.953,-15.619, \\
15.619,20.953,21.314,22.043,24.828,26.498,38.986,39.643\end{array}$ \\
\hline$A_{3}$ & $\begin{array}{l}-118.929,-116.959,-79.494,-74.485,-66.128,-63.942,-62.860,-58.925, \\
-58.816,-56.929,-56.785,-50.926,-50.325,-47.752,-47.710,-47.348, \\
-46.856,-46.658,-46.542,-46.305,-44.230,-43.348,-42.812,-42.332, \\
-42.159,-41.346,-40.694,-40.464,-40.269,-40.248,-39.413,-39.022, \\
-35.303,-34.448,-32.130,-31.349,-28.511,-26.507,-25.700,-25.385, \\
-24.585,-24.194,-23.443,-21.908,-20.938,-19.741,-16.681,-15.540, \\
-15.139,-14.620,-13.647,-13.026,-10.252,-8.594,-8.546,-8.396,-6.738, \\
-5.963,-4.566,-2.983,-1.841,-1.558,-1.258,-0.186,0.186,1.258,1.558, \\
1.841,2.983,4.566,5.963,6.738,8.396,8.546,8.594,10.252,13.026,13.647,14.620, \\
15.139,15.540,16.681,19.741,20.938,21.908,23.443,24.194,24.585,25.385, \\
25.700,26.507,28.511,31.349,32.130,34.448,35.303,39.022,39.413,40.248, \\
40.269,40.464,40.694,41.346,42.159,42.332,42.812,43.348,44.230,46.305, \\
46.542,46.658,46.856,47.348,47.710,47.752,50.325,50.926,56.785,56.929, \\
58.816,58.925,62.860,63.942,66.128,74.485,79.494,116.959,118.929\end{array}$ \\
\hline
\end{tabular}




\begin{tabular}{|c|c|}
\hline \multicolumn{2}{|c|}{$R=3.786 \quad \Lambda=-424.423 \quad \operatorname{SUSY}=(0,0) \quad \operatorname{dim}(H)=0$} \\
\hline$\Psi_{128}$ & 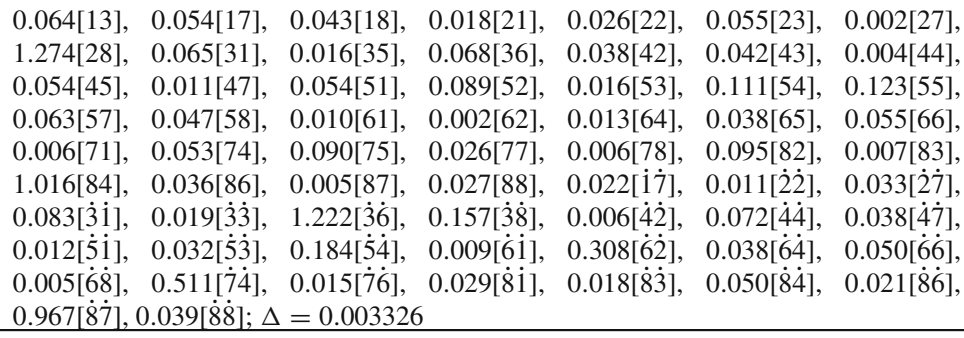 \\
\hline $\mathcal{M}_{s} / g^{2}$ & $\begin{array}{l}-535.110,-530.345,-511.629,-483.725,-418.777,-412.582,-332.040, \\
-325.466,-312.770,-308.735,-276.333,-255.891,-237.056,-231.357, \\
-230.672,-225.657,-188.937,-183.460,-182.919,-151.445,-113.355, \\
-108.155,-83.555,-73.582,-56.908,-43.036,-35.671,-3.886,-0.003(\times 4), \\
-0.002(\times 10),-0.001(\times 7), 0.000(\times 24), 0.001(\times 4), 0.002(\times 7), 38.996,148.877, \\
201.198,310.898,332.809,516.848,552.111,573.132,586.752,662.995,669.492, \\
680.384,730.094,823.170,832.767,924.009,1038.003,1056.777,1083.654, \\
1191.038,1223.112,1319.198,1324.058,1446.466,1447.623,1495.414,1607.268, \\
1654.783,1700.605,1725.723,1820.982,1890.025,1899.001,1926.976,1940.073, \\
1948.446,2006.914,2038.642,2319.796,2498.815,2732.826,2742.767,3024.765, \\
3064.001\end{array}$ \\
\hline $\mathcal{M}_{v} / g$ & $\begin{array}{l}-47.446,-47.318,-44.724,-44.385,-41.835,-41.698,-39.417,-39.331, \\
-37.492,-37.127,-36.838,-36.328,-36.089,-34.353,-32.660,-32.171, \\
-30.094,-28.491,-26.558,-23.885,-19.827,-18.762,-14.369,-12.862, \\
-9.860,-8.302,-4.386,-3.096,0.000(\times 72), 3.096,4.386,8.302,9.860,12.862, \\
14.369,18.762,19.827,23.885,26.558,28.490,30.094,32.171,32.660,34.353, \\
36.089,36.328,36.838,37.127,37.492,39.331,39.417,41.698,41.835,44.385, \\
44.724,47.318,47.446\end{array}$ \\
\hline$A_{1}$ & $\begin{array}{l}-35.652,-34.782,-29.084,-27.188,-25.409,-24.497,-19.825,-17.791, \\
17.791,19.825,24.497,25.409,27.188,29.084,34.782,35.652\end{array}$ \\
\hline$A_{3}$ & $\begin{array}{l}-106.956,-104.347,-87.251,-81.563,-76.226,-73.490,-59.475,-56.618, \\
-56.447,-53.372,-52.928,-52.591,-48.931,-48.020,-47.138,-46.863, \\
-46.257,-46.177,-45.459,-45.340,-44.732,-44.407,-43.887,-43.252, \\
-42.349,-41.759,-41.307,-41.273,-40.067,-39.563,-37.040,-34.824, \\
-34.563,-34.285,-32.127,-31.542,-30.776,-30.639,-28.485,-28.046, \\
-26.420,-24.463,-24.125,-22.865,-20.534,-20.204,-18.822,-18.306, \\
-17.684,-16.855,-14.979,-13.855,-12.005,-10.814,-9.844,-8.042,-7.802, \\
-7.548,-6.956,-4.955,-4.044,-3.272,-2.464,-2.317,2.317,2.464,3.272, \\
4.044,4.955,6.956,7.548,7.802,8.042,9.844,10.814,12.005,13.855,14.979, \\
16.855,17.684,18.306,18.822,20.204,20.534,22.865,24.125,24.463,26.420, \\
28.046,28.485,30.639,30.776,31.542,32.127,34.285,34.563,34.824,37.040, \\
39.563,40.067,41.273,41.307,41.759,42.349,43.252,43.887,44.407,44.732, \\
45.340,45.459,46.177,46.257,46.863,47.138,48.020,48.931,52.591,52.928, \\
53.372,56.447,56.618,59.475,73.490,76.226,81.563,87.251,104.347,106.956\end{array}$ \\
\hline
\end{tabular}

\begin{tabular}{|c|c|c|c|c|c|}
\hline \multicolumn{6}{|c|}{$R=3.790 \quad \Lambda=-432.430 \quad \operatorname{SUSY}=(0,0) \quad \operatorname{dim}(H)=0$} \\
\hline$\Psi_{128}$ & $\begin{array}{l}0.005[13] \\
0.068[46] \\
0.101[81] \\
0.144[\dot{2} \overline{7}] \\
0.012[\dot{5} \dot{2}] \\
0.002360\end{array}$ & $\begin{array}{cc}0.101[15], & 0.505[17], \\
0.056[48], & 0.143[56], \\
0.071[83], & 0.094[\dot{1} \dot{3}], \\
0.057[\dot{3} \dot{2}], & 0.229[\dot{3} \dot{6}], \\
0.047[\dot{5} \dot{6}], & 0.049[\dot{5} \dot{8}], 1\end{array}$ & $\begin{array}{c}0.505[21] \\
0.141[68] \\
0.018[\dot{1} \dot{5}] \\
0.047[\dot{3} \dot{8}] \\
133[\dot{6} \dot{2}], 0.0\end{array}$ & $\begin{array}{r}0.280[32], \\
0.005[71], \\
0.008[\dot{i}], \\
1.016[\dot{4} \dot{4}], \\
94[\dot{7} \dot{1}], 1.0\end{array}$ & $\begin{array}{l}0.143[38] \\
0.071[75] \\
1.086[\dot{2} \dot{3}] \\
0.040[\dot{4} \dot{8}] \\
{[\dot{8} \dot{1}] ; \Delta=}\end{array}$ \\
\hline
\end{tabular}




\begin{tabular}{|c|c|}
\hline & $\begin{array}{l}-556.682,-511.014,-474.645,-457.227,-428.334,-419.380,-409.277, \\
-390.568,-343.419,-336.611,-316.343,-311.542,-302.629,-272.429, \\
-265.220,-245.476,-230.534,-217.344,-208.648,-190.601,-180.279, \\
-150.778,-137.463,-117.881,-79.855,-44.686,-0.002(\times 3),-0.001(\times 5), \\
0.000(\times 35), 0.001(\times 6), 0.002(\times 7), 17.145,114.212,184.731,200.880,276.292, \\
294.143,313.336,525.604,544.287,581.624,615.948,672.493,690.015,774.206, \\
801.203,803.700,881.034,953.725,968.497,983.097,1104.853,1105.414, \\
1169.059,1218.905,1231.711,1236.409,1262.080,1326.071,1406.556,1413.946, \\
1593.509,1598.601,1664.198,1743.513,1771.315,1835.300,1867.773,1954.528, \\
2026.295,2031.669,2054.779,2101.044,2290.190,2308.853,2585.582,2645.298\end{array}$ \\
\hline & $\begin{array}{l}-45.918,-43.016,-42.837,-41.584,-40.693,-40.170,-39.480,-38.719, \\
-38.363,-38.315,-37.159,-36.643,-35.048,-34.870,-32.777,-30.383, \\
-29.118,-26.998,-24.926,-24.257,-19.864,-19.255,-16.267,-12.023, \\
-11.664,-11.220,-6.009,-3.926,0.000(\times 72), 3.926,6.009,11.220,11.664, \\
12.023,16.267,19.255,19.864,24.257,24.926,26.998,29.118,30.383,32.777, \\
34.870,35.048,36.643,37.159,38.315,38.363,38.719,39.480,40.170,40.693, \\
41.584,42.837,43.016,45.918\end{array}$ \\
\hline$A_{1}$ & $\begin{array}{l}352,-20.041,-19.621, \\
4.794\end{array}$ \\
\hline$A_{3}$ & $\begin{array}{l}-104.382,-100.910,-85.858,-77.216,-76.147,-73.056,-60.122,-58.862, \\
-51.405,-50.703,-50.143,-49.668,-48.014,-47.837,-46.956,-46.923, \\
-46.008,-45.824,-45.025,-44.508,-44.098,-43.810,-43.287,-42.401, \\
-41.993,-40.198,-39.866,-38.568,-37.934,-37.554,-36.024,-35.435, \\
-34.893,-33.628,-33.054,-31.531,-30.152,-29.532,-29.266,-28.426, \\
-25.476,-24.079,-24.062,-23.156,-21.870,-21.550,-18.688,-17.862, \\
-16.279,-15.633,-14.052,-13.667,-13.341,-12.463,-10.616,-10.383, \\
-7.679,-7.426,-5.397,-4.903,-3.525,-3.476,-1.869,-0.420,0.420,1.869, \\
3.476,3.525,4.903,5.397,7.426,7.679,10.383,10.616,12.463,13.341,13.667, \\
14.052,15.633,16.279,17.862,18.688,21.550,21.870,23.156,24.062,24.079, \\
25.476,28.426,29.266,29.532,30.152,31.531,33.054,33.628,34.893,35.435, \\
36.024,37.554,37.934,38.568,39.866,40.198,41.993,42.401,43.287,43.810, \\
44.098,44.508,45.025,45.824,46.008,46.923,46.956,47.837,48.014,49.668, \\
50.143,50.703,51.405,58.862,60.122,73.056,76.147,77.216,85.858,100.910, \\
104.382\end{array}$ \\
\hline
\end{tabular}

\begin{tabular}{|c|c|}
\hline \multicolumn{2}{|c|}{$R=3.795 \quad \Lambda=-422.755 \quad \operatorname{SUSY}=(0,0) \quad \operatorname{dim}(H)=0$} \\
\hline$\Psi_{128}$ & 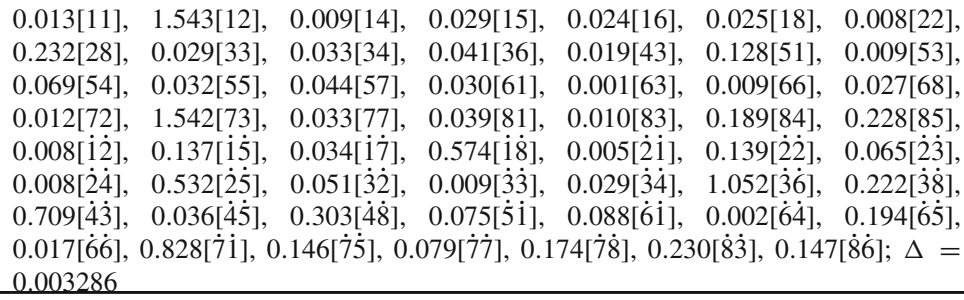 \\
\hline $\mathcal{M}_{s} / g^{2}$ & $\begin{array}{l}-641.295,-609.372,-508.647,-465.567,-400.787(\times 2),-350.815,-349.893, \\
-346.853,-343.384(\times 2),-328.738,-294.493(\times 2),-272.252,-229.166(\times 2), \\
-197.098,-116.830,-113.668,-82.566(\times 2),-73.205,-56.203,-48.912, \\
-0.004,-0.003(\times 3),-0.002(\times 10),-0.001(\times 8), 0.000(\times 21), 0.001(\times 4), \\
0.002(\times 7), 0.003(\times 2), 52.214,273.917,273.918,430.798,440.811(\times 2), 464.544, \\
464.545,477.219,578.006,727.233,832.937,832.939,856.213,919.400,937.748, \\
955.845,1012.849,1025.752,1054.671(\times 2), 1174.553,1174.555,1299.465, \\
1299.467,1342.483,1381.389,1381.391,1452.198,1772.856,1908.987,1910.302, \\
1988.014,2043.011,2047.879,2221.394,2236.950,2301.981,2301.983,2309.693, \\
2309.696,2918.778,2968.480,3463.941(\times 2), 4554.754,4568.034\end{array}$ \\
\hline
\end{tabular}




\begin{tabular}{|c|c|}
\hline $\mathcal{M}_{v / g}$ & $\begin{array}{l}-52.730,-52.647,-50.687,-50.279,-44.660,-42.122,-40.908,-40.306, \\
-39.477,-38.853,-37.549,-36.262,-34.888,-34.125,-33.437,-31.185, \\
-30.835,-30.568,-29.135,-25.759,-23.440,-20.340,-19.827,-8.133, \\
-6.626,-5.527,-3.681,-2.113,0.000(\times 72), 2.113,3.681,5.527,6.626,8.133, \\
19.827,20.340,23.440,25.759,29.135,30.568,30.835,31.185,33.437,34.125, \\
34.888,36.262,37.549,38.853,39.477,40.306,40.908,42.122,44.660,50.279, \\
50.687,52.647,52.730\end{array}$ \\
\hline$A_{1}$ & $\begin{array}{l}-37.913(\times 2),-27.078(\times 2),-25.752(\times 2),-21.580(\times 2), 21.580(\times 2), 25.752(\times 2), \\
27.078(\times 2), 37.913(\times 2)\end{array}$ \\
\hline$A_{3}$ & 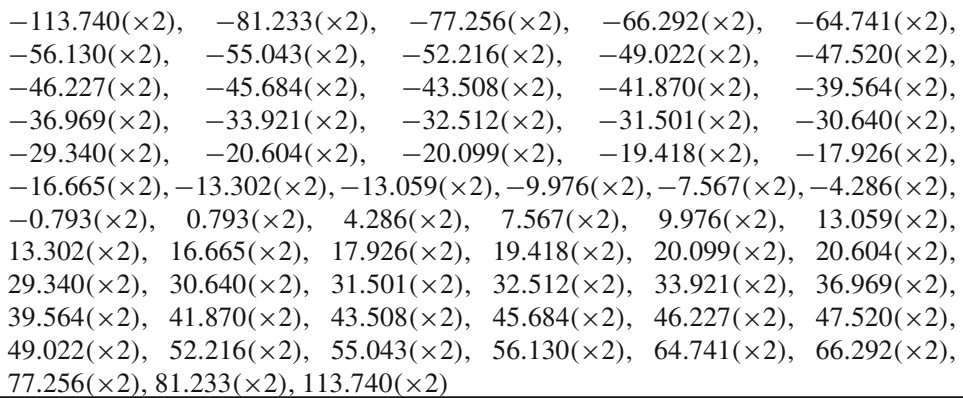 \\
\hline
\end{tabular}

\begin{tabular}{|c|c|}
\hline \multicolumn{2}{|c|}{$R=3.840 \quad \Lambda=-458.646 \quad \operatorname{SUSY}=(0,0) \quad \operatorname{dim}(H)=0$} \\
\hline$\Psi_{128}$ & 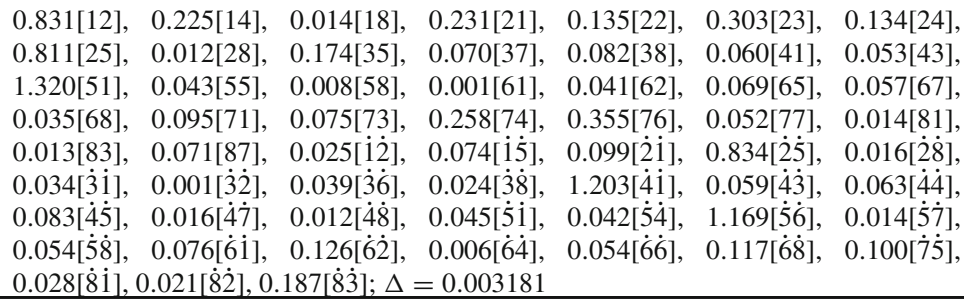 \\
\hline $\mathcal{M}_{s} / g^{2}$ & $\begin{array}{l}-554.347,-524.830,-468.441,-450.955,-433.773,-420.354,-391.248, \\
-377.368,-361.410,-338.980,-334.718,-283.126,-274.151,-250.731, \\
-234.106,-203.629,-182.157,-164.204,-145.717,-124.392,-102.025, \\
-93.931,-74.487,-59.567,-35.126,-4.371,-0.004(\times 3),-0.003(\times 6), \\
-0.002(\times 8),-0.001(\times 4), 0.000(\times 19), 0.001(\times 4), 0.002(\times 7), 0.003(\times 4), 0.004, \\
53.817,77.575,138.064,153.193,277.790,312.176,379.891,517.918,597.390, \\
629.330,688.996,730.889,898.962,916.319,970.833,1095.642,1155.893, \\
1182.861,1225.276,1311.529,1318.731,1413.413,1462.226,1492.828,1597.636, \\
1653.065,1708.471,1782.557,1832.613,1880.842,1945.998,2003.650,2066.169, \\
2083.167,2175.220,2208.742,2331.583,2405.708,2452.594,2510.165,2593.439, \\
2627.190,3334.366,3337.055,3639.883,3647.393\end{array}$ \\
\hline $\mathcal{M}_{v} / g$ & $\begin{array}{l}-49.817,-49.573,-46.321,-46.091,-45.230,-44.341,-43.525,-43.367, \\
-40.917,-39.477,-38.891,-38.662,-38.059,-35.792,-34.576,-33.263, \\
-31.077,-30.919,-28.410,-27.475,-22.870,-22.476,-20.230,-16.719, \\
-9.983,-7.815,-4.522,-3.543,0.000(\times 72), 3.741,4.554,8.744,13.258,15.432, \\
18.350,19.357,24.233,26.409,27.436,30.396,30.889,32.252,34.643,36.082, \\
36.720,37.125,38.240,38.544,40.078,41.222,42.527,43.356,45.325,48.654, \\
49.235,53.443,53.692\end{array}$ \\
\hline$A_{1}$ & $\begin{array}{l}-36.664,-35.810,-31.551,-29.173,-27.569,-24.854,-22.351,-20.911, \\
19.530,20.240,25.699,27.159,28.689,30.940,38.089,38.536\end{array}$ \\
\hline
\end{tabular}




\begin{tabular}{|c|c|}
\hline$A_{3}$ & $\begin{array}{l}-109.991,-107.430,-94.652,-87.520,-82.707,-74.561,-67.054,-62.732, \\
-60.831,-60.657,-56.981,-56.852,-51.684,-50.759,-50.297,-50.059, \\
-49.213,-48.912,-48.492,-47.967,-47.430,-47.188,-47.081,-45.457, \\
-44.739,-44.193,-43.953,-43.703,-42.747,-42.137,-42.125,-40.223, \\
-38.430,-37.015,-35.357,-34.542,-33.477,-33.270,-31.539,-30.130, \\
-28.534,-28.172,-27.235,-24.951,-23.899,-23.602,-22.062,-20.320, \\
-19.936,-17.822,-15.493,-13.855,-13.000,-12.475,-11.439,-8.659, \\
-8.374,-6.673,-5.884,-5.052,-4.234,-3.924,-1.178,-0.293,1.010,2.851, \\
4.033,4.873,5.088,5.539,6.145,7.309,9.363,11.052,12.431,13.242,15.488, \\
16.243,18.198,19.527,20.851,22.285,23.048,24.058,24.262,26.250,26.547, \\
28.091,29.016,30.714,32.450,32.964,33.696,34.447,36.042,37.095,38.489, \\
39.393,40.728,42.044,43.045,43.679,45.013,45.448,46.436,46.871,47.129, \\
47.432,47.863,49.060,49.929,50.264,51.227,52.118,53.514,54.042,54.527, \\
54.985,58.591,60.720,63.503,63.552,77.098,81.478,86.066,92.820,114.267, \\
115.608\end{array}$ \\
\hline
\end{tabular}

\begin{tabular}{|c|c|}
\hline \multicolumn{2}{|c|}{$R=3.869 \quad \Lambda=-467.885 \quad \operatorname{SUSY}=(0,0) \quad \operatorname{dim}(H)=0$} \\
\hline$\Psi_{128}$ & 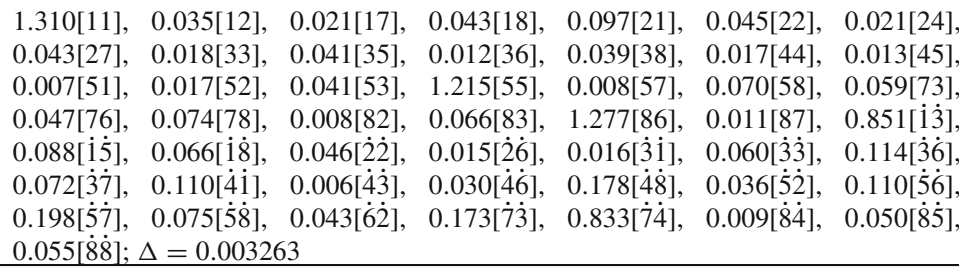 \\
\hline $\mathcal{M}_{s} / g^{2}$ & $\begin{array}{l}-914.735,-814.723,-696.464,-643.547,-643.546,-520.683,-459.321, \\
-455.145(\times 2),-408.151(\times 2),-335.289,-273.567,-163.528(\times 2),-97.202, \\
-59.541,-57.056,-57.055,-0.003(\times 5),-0.002(\times 9),-0.001(\times 4), 0.000(\times 24), \\
0.001(\times 5), 0.002(\times 7), 0.003(\times 2), 41.851(\times 2), 69.179,69.180,88.088,253.898, \\
253.899,439.989,439.990,518.070,681.730,738.608,738.610,761.083,762.913, \\
762.915,808.272(\times 2), 832.903,832.904,995.033,1002.143,1002.144,1112.879, \\
1112.882,1118.977,1136.016,1155.314,1155.317,1195.934,1195.935,1302.248, \\
1641.108,1778.849,1778.851,1894.819,1894.821,2129.574,2245.791,2245.793, \\
2305.696,2437.314,2437.316,2442.901,2442.902,2450.461,2487.726,2487.728, \\
2678.033,2678.037,2962.397,3181.256,3411.001\end{array}$ \\
\hline $\mathcal{M}_{v} / g$ & $\begin{array}{l}-51.507,-49.031,-48.078,-47.254(\times 2),-41.390,-41.086(\times 2),-38.302(\times 2), \\
-37.820,-37.299,-36.562(\times 2),-35.897,-34.959(\times 2),-32.976(\times 2), \\
-28.782(\times 2),-27.393,-26.938,-11.710,-9.356,-5.419,-3.426,-2.572, \\
0.000(\times 72), 2.572,3.426,5.419,9.356,11.710,26.938,27.393,28.782(\times 2), \\
32.976(\times 2), 34.959(\times 2), 35.897,36.562(\times 2), 37.299,37.820,38.302(\times 2), \\
41.086(\times 2), 41.390,47.254(\times 2), 48.078,49.031,51.507\end{array}$ \\
\hline$A_{1}$ & $-33.926(\times 4),-24.550(\times 4), 24.550(\times 4), 33.926(\times 4)$ \\
\hline$A_{3}$ & $\begin{array}{l}-101.777(\times 4),-73.651,-73.650(\times 3),-56.967(\times 4),-50.727(\times 4),-49.003(\times 4), \\
-46.759(\times 3),-46.758,-45.974(\times 4),-44.160(\times 4),-40.617(\times 4),-30.080(\times 4), \\
-26.719(\times 4), \quad-18.422(\times 4), \quad-17.833(\times 4), \quad-16.029(\times 4),-12.644(\times 4), \\
-8.383(\times 4), 8.383(\times 4), 12.644(\times 4), 16.029(\times 4), 17.833(\times 4), 18.422(\times 4), \\
26.719(\times 4), 30.080(\times 4), 40.617(\times 4), 44.160(\times 4), 45.974(\times 4), 46.758,46.759(\times 3), \\
49.003(\times 4), 50.727(\times 4), 56.967(\times 4), 73.650(\times 2), 73.651(\times 2), 101.777(\times 4)\end{array}$ \\
\hline
\end{tabular}




\begin{tabular}{|c|c|}
\hline \multicolumn{2}{|c|}{$R=3.874 \quad \Lambda=-482.518 \quad \operatorname{SUSY}=(0,0) \quad \operatorname{dim}(H)=0$} \\
\hline$\Psi_{128}$ & 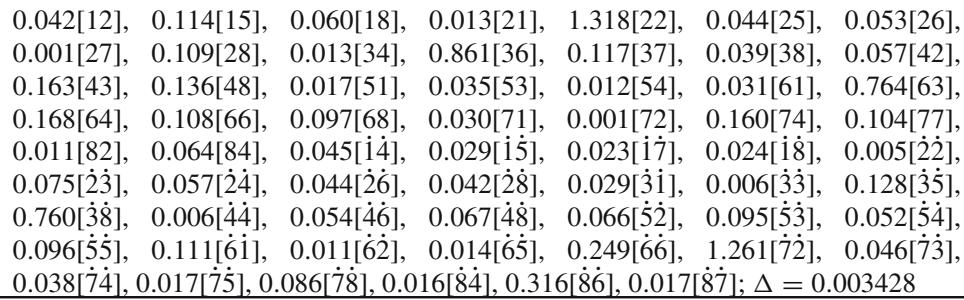 \\
\hline $\mathcal{M}_{s} / g^{2}$ & $\begin{array}{l}-571.630,-549.068,-545.985,-470.376,-448.033,-413.115,-392.326, \\
-376.083,-373.426,-342.276,-316.470,-303.554,-265.152,-261.417, \\
-241.860,-226.871,-203.045,-178.438,-162.844,-131.787,-125.505, \\
-93.867,-74.251,-51.679,-17.563,-0.005,-0.004(\times 5),-0.003(\times 4), \\
-0.002(\times 5),-0.001(\times 6), 0.000(\times 18), 0.001(\times 5), 0.002(\times 5), 0.003(\times 5), \\
0.004(\times 2), 68.056,82.663,139.363,167.991,203.716,272.633,393.213,417.903, \\
608.611,658.276,755.507,845.088,900.754,975.247,1004.644,1088.255, \\
1120.957,1222.253,1258.495,1332.506,1343.453,1376.927,1447.747,1501.049, \\
1531.400,1586.139,1733.606,1815.054,1872.384,1924.053,1983.796,2004.347, \\
2090.727,2125.710,2155.443,2244.739,2272.772,2334.704,2409.331,2447.610, \\
2570.341,2809.477,2831.383,3576.700,3601.255,3853.227,3862.733\end{array}$ \\
\hline $\mathcal{M}_{v} / g$ & $\begin{array}{l}-53.651,-53.376,-48.583,-47.679,-46.438,-44.703,-43.700,-42.286, \\
-41.479,-40.920,-40.700,-39.701,-39.273,-37.540,-35.381,-34.274, \\
-31.511,-30.727,-29.718,-26.793,-25.989,-21.431,-19.481,-15.860, \\
-11.990,-8.636,-5.859,-4.728,0.000(\times 72), 2.504,6.290,9.092,13.287,16.089, \\
20.746,23.353,25.798,29.394,30.032,30.781,33.362,35.747,36.544,36.934, \\
37.544,38.121,39.094,39.427,39.534,41.152,42.166,45.047,45.197,49.804, \\
50.179,52.409,52.780\end{array}$ \\
\hline$A_{1}$ & $\begin{array}{l}-37.820,-37.323,-33.709,-31.470,-27.935,-25.210,-21.788,-20.350, \\
19.698,22.510,27.119,29.478,31.480,32.852,35.824,36.646\end{array}$ \\
\hline$A_{3}$ & $\begin{array}{l}-113.459,-111.970,-101.128,-94.410,-83.806,-75.630,-65.365,-64.096, \\
-64.043,-61.050,-56.330,-56.042,-54.714,-54.157,-52.882,-52.344, \\
-51.779,-51.279,-50.031,-49.313,-48.922,-48.393,-47.057,-46.761, \\
-46.348,-45.445,-44.614,-44.420,-43.763,-42.389,-41.580,-40.018, \\
-39.018,-37.595,-36.626,-35.765,-34.336,-33.458,-31.637,-31.296, \\
-29.785,-29.004,-27.477,-25.819,-25.517,-22.980,-22.299,-21.236, \\
-20.387,-19.218,-17.580,-15.186,-13.939,-11.886,-11.075,-10.480, \\
-9.116,-8.456,-7.109,-6.450,-4.139,-3.517,-2.763,-0.580,0.742,2.480, \\
2.966,5.107,5.526,7.072,8.058,8.373,10.073,11.122,13.109,14.459,16.379, \\
17.846,18.732,19.845,21.804,23.151,24.287,25.971,26.411,26.938,28.675, \\
30.524,32.003,32.345,34.255,35.540,35.989,36.572,38.164,39.133,39.632, \\
40.624,41.335,43.458,44.560,44.826,45.478,46.256,46.913,47.188,47.454, \\
48.360,48.715,49.759,50.549,50.947,51.828,52.272,53.725,53.884,55.615, \\
55.841,59.093,64.777,64.807,67.529,81.356,88.434,94.440,98.557,107.473,\end{array}$ \\
\hline
\end{tabular}




\begin{tabular}{|c|c|}
\hline \multicolumn{2}{|c|}{$R=3.928 \quad \Lambda=-518.952 \quad \operatorname{SUSY}=(0,0) \quad \operatorname{dim}(H)=0$} \\
\hline$\Psi_{128}$ & 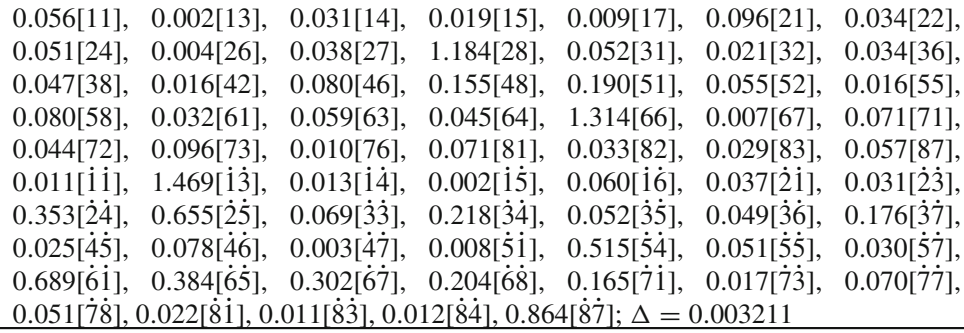 \\
\hline $\mathcal{M}_{s} / g^{2}$ & $\begin{array}{l}-662.632,-606.814,-591.850,-503.488,-485.813,-460.017,-435.509, \\
-420.492,-394.490,-342.900,-329.368,-284.364,-257.875,-241.723, \\
-225.920,-202.402,-162.470,-135.604,-108.413,-78.833,-55.198,-42.043, \\
-38.145,-5.874,-5.480,-5.174,-4.739,-4.519,-4.409,-4.268,-3.906, \\
-3.653,-3.520,-3.254,-3.133,-2.996,-2.553,-2.141,-1.914,-1.676, \\
-1.625,-1.376,-1.246,-1.146,-0.955,-0.607,-0.530,-0.140,-0.126, \\
-0.061,-0.034,-0.019,0.257,0.428,0.503,0.606,0.771,0.931,1.086,1.252, \\
1.411,1.742,1.920,2.133,2.409,2.520,2.778,2.814,3.020,3.314,3.626,3.797, \\
4.004,4.368,4.614,4.720,4.963,5.472,5.987,6.611,93.932,220.166,226.772, \\
246.973,361.421,385.521,548.180,576.850,662.698,742.647,826.196,947.326, \\
969.098,1084.856,1109.092,1278.279,1329.430,1349.089,1474.320,1547.815, \\
1596.541,1702.603,1803.265,1898.462,1981.324,2056.270,2118.097,2180.834, \\
2232.921,2304.562,2389.664,2443.780,2483.971,2534.911,2597.847,2652.048, \\
2685.061,2740.011,2990.306,3081.130,3359.213,3383.558,3730.889,3750.295, \\
4813.945,4826.030,5993.274,6000.164\end{array}$ \\
\hline $\mathcal{M}_{v} / g$ & $\begin{array}{l}-61.654,-61.563,-54.857,-54.585,-52.285,-50.763,-49.597,-47.696, \\
-45.100,-43.903,-43.519,-41.589,-39.601,-39.404,-37.572,-36.811, \\
-36.236,-34.604,-31.396,-29.984,-26.999,-26.777,-22.769,-17.348, \\
-11.659,-7.851,-4.970,-3.832,0.000(\times 72), 2.787,7.047,7.803,11.522,17.558, \\
22.090,26.854,27.996,30.406,32.558,32.927,36.026,36.778,37.563,39.821, \\
40.097,41.774,43.461,44.056,45.474,47.052,49.071,51.596,52.117,53.477, \\
53.641,61.666,61.706\end{array}$ \\
\hline$A_{1}$ & $\begin{array}{l}-42.213,-41.344,-34.646,-32.885,-31.047,-29.934,-26.018,-20.039, \\
20.123,26.779,29.239,31.826,32.701,35.641,39.924,41.895\end{array}$ \\
\hline$A_{3}$ & $\begin{array}{l}-126.639,-124.033,-103.939,-98.655,-93.144,-89.803,-78.056,-76.302, \\
-76.275,-65.837,-65.787,-62.098,-61.818,-60.119,-60.077,-59.951, \\
-58.164,-57.308,-54.440,-54.074,-53.208,-51.905,-51.706,-51.130, \\
-50.133,-49.610,-48.772,-47.721,-46.658,-46.037,-45.487,-44.191, \\
-43.520,-40.958,-39.462,-37.944,-37.517,-35.836,-34.806,-33.452, \\
-31.652,-30.918,-30.067,-28.673,-27.542,-27.193,-24.962,-23.692, \\
-22.923,-21.806,-21.386,-19.410,-18.071,-14.727,-14.356,-11.753, \\
-11.188,-9.642,-6.610,-6.501,-5.107,-3.999,-2.338,-1.581,2.594,2.926, \\
3.850,4.199,5.475,7.585,8.154,12.075,12.861,13.281,13.675,17.761,18.789, \\
20.454,22.384,23.764,25.010,25.434,26.713,28.223,29.860,30.491,31.635, \\
32.507,33.664,35.433,37.107,37.218,38.004,38.968,41.428,42.844,44.158, \\
45.161,46.057,46.458,46.884,48.236,49.272,49.639,49.940,50.834,51.239, \\
53.339,53.462,54.394,57.626,58.200,59.983,60.191,60.371,61.743,62.044, \\
66.506,66.627,75.943,75.980,80.337,87.712,95.477,98.102,106.925,119.776, \\
125.686\end{array}$ \\
\hline
\end{tabular}




\begin{tabular}{|c|c|}
\hline \multicolumn{2}{|c|}{$R=4.002 \quad \Lambda=-576.638 \quad \operatorname{SUSY}=(0,0) \quad \operatorname{dim}(H)=0$} \\
\hline$\Psi_{128}$ & 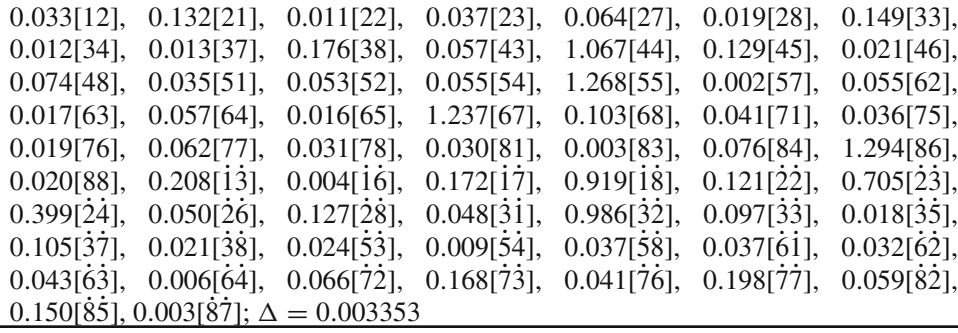 \\
\hline $\mathcal{M}_{s} / g^{2}$ & $\begin{array}{l}-831.670,-799.814,-679.553,-653.255,-580.430,-548.964,-522.714, \\
-498.781,-472.682,-446.044,-350.141,-349.392,-310.190,-307.370, \\
-267.899,-258.256,-243.818,-179.491,-129.565,-88.608,-69.294,-60.623, \\
-57.939,-0.005(\times 2),-0.004(\times 2),-0.003(\times 4),-0.002(\times 6),-0.001(\times 2), \\
0.000(\times 15), 0.001(\times 3), 0.002(\times 4), 0.003(\times 6), 0.004(\times 4), 0.005(\times 4), 0.006(\times 2), \\
0.007(\times 2), 107.954,108.774,149.880,182.752,207.686,367.307,638.292,713.372, \\
821.964,1014.650,1049.354,1142.430,1162.564,1204.270,1276.339,1297.169, \\
1371.085,1489.055,1552.822,1584.342,1615.302,1957.345,1977.680,2191.567, \\
2204.060,2308.275,2335.258,2392.975,2412.170,2497.124,2642.759,2654.378, \\
2668.353,2688.564,2699.926,2733.981,2794.213,3807.408,3888.145,4127.823, \\
4189.424,4304.564,4315.281,4585.224,4598.888,4876.970,4883.549,5359.759, \\
5372.626\end{array}$ \\
\hline $\mathcal{M}_{v} / g$ & $\begin{array}{l}-70.737,-70.714,-56.393,-56.056,-55.817,-55.533,-49.260,-48.772, \\
-48.344,-46.549,-44.982,-41.351,-40.794,-40.574,-38.569,-37.952, \\
-37.906,-37.467,-34.198,-33.338,-30.857,-24.105,-19.606,-18.800, \\
-13.658,-10.884,-5.952,-2.607,0.000(\times 72), 2.607,5.952,10.884,13.658, \\
18.800,19.606,24.105,30.857,33.338,34.197,37.467,37.906,37.952,38.569, \\
40.574,40.794,41.351,44.982,46.549,48.344,48.772,49.260,55.533,55.817, \\
56.056,56.393,70.714,70.737\end{array}$ \\
\hline$A_{1}$ & $\begin{array}{l}-50.873,-50.647,-34.436,-34.310,-24.147,-24.022,-23.656,-23.430, \\
23.430,23.656,24.022,24.147,34.310,34.436,50.647,50.873\end{array}$ \\
\hline$A_{3}$ & $\begin{array}{l}-152.619,-151.940,-103.307,-102.931,-73.824,-73.812,-73.760,-73.750, \\
-72.440,-72.065,-70.969,-70.311,-70.290,-70.163,-66.762,-66.664, \\
-60.851,-60.709,-57.080,-57.073,-57.069,-57.045,-51.445,-51.245, \\
-50.511,-50.475,-49.775,-48.595,-48.379,-48.055,-46.800,-46.129, \\
-46.081,-45.256,-43.104,-42.852,-42.246,-41.956,-41.493,-39.893, \\
-35.333,-34.365,-34.298,-33.233,-30.972,-30.063,-28.542,-28.007, \\
-19.890,-16.178,-15.975,-15.159,-14.747,-13.769,-13.757,-11.800, \\
-10.104,-5.522,-5.508,-3.988,-3.719,-2.605,-2.569,-2.223,2.223,2.569, \\
2.605,3.719,3.988,5.508,5.522,10.104,11.800,13.757,13.769,14.747,15.159, \\
15.975,16.178,19.890,28.007,28.542,30.063,30.972,33.233,34.298,34.365, \\
35.333,39.893,41.493,41.956,42.246,42.852,43.105,45.256,46.081,46.129, \\
46.800,48.055,48.379,48.595,49.775,50.475,50.511,51.245,51.445,57.045, \\
57.069,57.073,57.080,60.709,60.851,66.664,66.762,70.163,70.290,70.311, \\
70.969,72.065,72.441,73.750,73.760,73.812,73.824,102.931,103.307,151.940,\end{array}$ \\
\hline
\end{tabular}




\begin{tabular}{|c|c|}
\hline \multicolumn{2}{|c|}{$R=4.032 \quad \Lambda=-606.053 \quad \operatorname{SUSY}=(0,0) \quad \operatorname{dim}(H)=0$} \\
\hline & 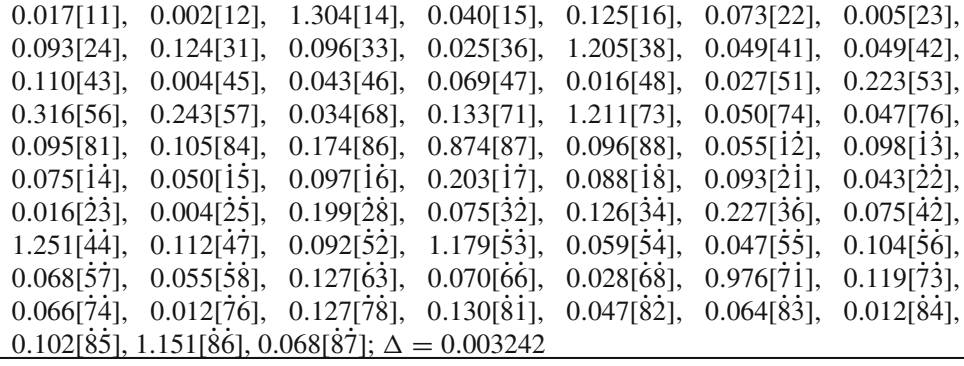 \\
\hline & $\begin{array}{l}-782.673,-746.307,-664.530,-616.986,-587.664,-551.916,-518.606, \\
-484.145,-468.816,-429.152,-361.123,-341.081,-305.181,-289.659, \\
-203.053,-154.378,-140.501,-131.578,-90.893,-72.132,-54.113, \\
-0.005(\times 2),-0.004(\times 3),-0.003(\times 7),-0.002(\times 7),-0.001(\times 3), 0.000(\times 17), \\
0.001(\times 3), 0.002(\times 5), 0.003(\times 6), 0.004(\times 3), 43.991,94.460,148.183,243.485, \\
345.670,439.915,558.860,639.343,688.150,706.016,809.136,951.020,985.310, \\
1069.563,1126.856,1371.319,1431.359,1480.719,1642.200,1713.638,1852.947, \\
1867.030,1909.328,2018.405,2078.937,2180.420,2304.047,2402.240,2480.545, \\
2530.111,2553.148,2662.117,2756.986,2769.964,2823.578,2853.739,2982.918, \\
3071.118,3149.429,3232.881,3421.498,3511.841,3671.606,3771.738,3869.335, \\
4217.134,4248.790,4706.792,4720.405,4986.307,4993.978\end{array}$ \\
\hline $\mathcal{M}_{v} / g$ & $\begin{array}{l}-62.559,-62.315,-57.188,-56.531,-55.621,-54.765,-53.004,-50.826, \\
-49.372,-48.123,-47.181,-46.108,-43.799,-42.704,-41.079,-39.293, \\
-38.562,-37.444,-36.774,-32.789,-30.797,-30.362,-26.454,-24.955, \\
-21.317,-12.638,-6.555,-3.544,0.000(\times 72), 5.765,7.388,11.094,18.280, \\
25.454,27.756,30.918,31.390,33.752,36.687,38.767,39.155,40.437,42.093, \\
43.501,44.175,45.817,45.875,47.068,49.405,50.053,51.590,53.251,55.677, \\
57.150,57.445,61.233,61.484\end{array}$ \\
\hline$A_{1}$ & $\begin{array}{l}-45.357,-42.390,-38.890,-36.527,-32.393,-31.455,-26.959,-25.579, \\
25.799,28.595,31.384,32.655,37.159,38.820,41.900,43.235\end{array}$ \\
\hline$A_{3}$ & $\begin{array}{l}-136.072,-127.170,-116.669,-109.580,-97.178,-94.364,-80.876,-76.736, \\
-72.757,-72.701,-67.752,-67.238,-65.005,-64.172,-63.226,-62.763, \\
-61.952,-61.299,-60.038,-59.566,-58.760,-57.937,-56.819,-56.265, \\
-55.199,-54.263,-53.323,-52.374,-51.864,-50.494,-49.690,-48.839, \\
-48.411,-47.661,-45.594,-42.337,-41.260,-40.324,-39.406,-38.445, \\
-36.503,-35.682,-34.624,-33.200,-31.002,-29.841,-28.284,-27.902, \\
-25.872,-24.883,-24.837,-22.006,-20.355,-19.792,-16.242,-14.068, \\
-12.438,-10.226,-9.217,-6.761,-5.072,-3.809,-2.899,0.285,0.620,2.688, \\
4.460,5.022,7.185,8.624,10.601,12.400,15.096,15.846,18.118,19.874,20.826, \\
22.824,24.634,26.308,28.262,29.929,31.178,31.943,32.509,34.077,36.109, \\
37.927,39.018,40.920,41.152,41.391,43.892,45.779,45.838,48.161,49.650, \\
50.648,51.403,51.752,52.689,53.751,54.271,54.900,55.941,56.266,57.647, \\
58.257,58.551,59.114,60.546,60.931,62.234,62.845,64.546,64.923,65.976, \\
66.375,73.240,73.299,77.398,85.786,94.152,97.966,111.476,116.461,125.701, \\
129.706\end{array}$ \\
\hline
\end{tabular}




\begin{tabular}{|c|c|}
\hline \multicolumn{2}{|c|}{$R=4.051 \quad \Lambda=-615.137 \quad \operatorname{SUSY}=(0,0) \quad \operatorname{dim}(H)=0$} \\
\hline$\Psi_{128}$ & 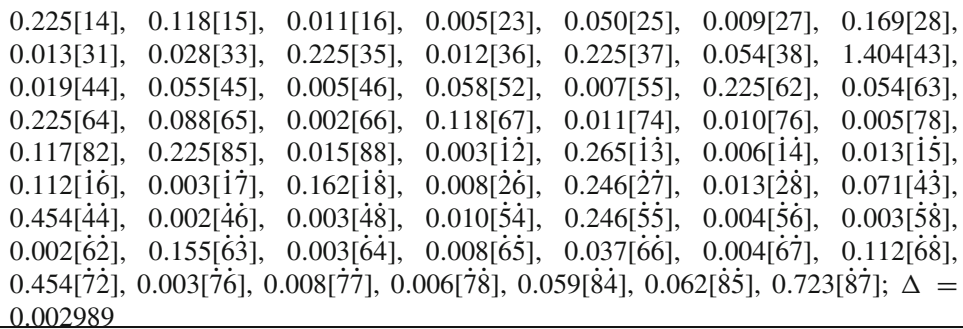 \\
\hline $\mathcal{M}_{s}$ & $\begin{array}{l}-1135.092,-884.886,-800.552,-718.801,-691.859,-502.286,-450.665, \\
-423.632,-411.613,-177.439,-137.351,-92.916,-82.609,-71.239,-71.045, \\
-68.233,-38.160,-36.960,-21.220,-0.008,-0.007(\times 5),-0.006(\times 3),-0.005, \\
-0.004,-0.003(\times 2),-0.002,-0.001(\times 2), 0.000(\times 14), 0.001(\times 4), 0.002(\times 6), \\
0.003(\times 4), 0.005(\times 3), 0.006(\times 2), 0.007(\times 3), 0.008(\times 2), 0.009(\times 2), 16.551, \\
84.585,100.157,352.068,367.872,509.967,524.777,585.582,624.578,847.861, \\
857.951,886.128,1028.077,1052.753,1298.959,1447.174,1553.753,1656.976, \\
1764.956,2031.575,2181.910,2434.240,2608.781,2613.650,2716.649,2768.746, \\
2781.734,2782.976,2844.517,3035.488,3218.826,3252.824,3253.795,3260.842, \\
3329.792,3393.732,3436.546,3487.614,3559.156,4112.162,4229.308,4823.044, \\
4857.255,6347.689,6356.539,7260.392,7261.029,7531.941,7535.313,8012.429, \\
8014.290,12371.244,12371.710\end{array}$ \\
\hline $\mathcal{M}_{v} / g$ & $\begin{array}{l}-85.913,-85.910,-66.972,-66.806,-64.582,-64.161,-60.963,-60.836, \\
-60.249,-59.971,-49.019,-48.316,-48.213,-45.219,-44.919,-44.396, \\
-41.213,-39.544,-38.757,-37.137,-34.268,-34.016,-32.656,-29.782, \\
-7.935,-5.358,-4.642,-1.430,0.000(\times 72), 1.430,4.642,5.358,7.935,29.782, \\
32.656,34.016,34.268,37.137,38.757,39.544,41.213,44.396,44.919,45.219, \\
48.213,48.316,49.019,59.971,60.249,60.836,60.963,64.161,64.582,66.806, \\
66.972,85.910,85.913\end{array}$ \\
\hline$A_{1}$ & $\begin{array}{l}-48.112(\times 2),-47.369(\times 2),-32.873(\times 2),-32.129(\times 2), 32.129(\times 2), 32.873(\times 2), \\
47.369(\times 2), 48.112(\times 2)\end{array}$ \\
\hline$A_{3}$ & $\begin{array}{l}-144.337(\times 2),-142.107(\times 2),-101.138(\times 4),-98.618(\times 2),-96.388(\times 2), \\
-76.022(\times 2),-75.983(\times 2),-72.872(\times 2),-72.833(\times 2),-64.921,-64.920, \\
-64.448(\times 2),-59.419(\times 2),-58.288(\times 2),-54.131(\times 2),-54.043(\times 2),-48.487, \\
-48.486,-48.423(\times 2),-45.885(\times 2),-44.988(\times 2),-42.603(\times 2),-42.256(\times 2), \\
-30.262(\times 2),-28.459(\times 2),-23.024(\times 2),-22.947(\times 2),-18.953(\times 2), \\
-18.705(\times 2),-18.043(\times 2),-17.736(\times 2),-11.035(\times 2),-8.017(\times 2), 8.017(\times 2), \\
11.035(\times 2), 17.736(\times 2), 18.043(\times 2), 18.705(\times 2), 18.953(\times 2), 22.947(\times 2), \\
23.024(\times 2), 28.459(\times 2), 30.262(\times 2), 42.256(\times 2), 42.603(\times 2), 44.988(\times 2), \\
45.885(\times 2), 48.423(\times 2), 48.486,48.487,54.043(\times 2), 54.131(\times 2), 58.288(\times 2), \\
59.419(\times 2), 64.448(\times 2), 64.920(\times 2), 72.833(\times 2), 72.872(\times 2), 75.983(\times 2), \\
76.022(\times 2), 96.388(\times 2), 98.618(\times 2), 101.138(\times 4), 142.107(\times 2), 144.336,144.337\end{array}$ \\
\hline
\end{tabular}




\begin{tabular}{|c|c|}
\hline \multicolumn{2}{|c|}{$R=4.075 \quad \Lambda=-635.266 \quad \operatorname{SUSY}=(0,0) \quad \operatorname{dim}(H)=1$} \\
\hline 28 & 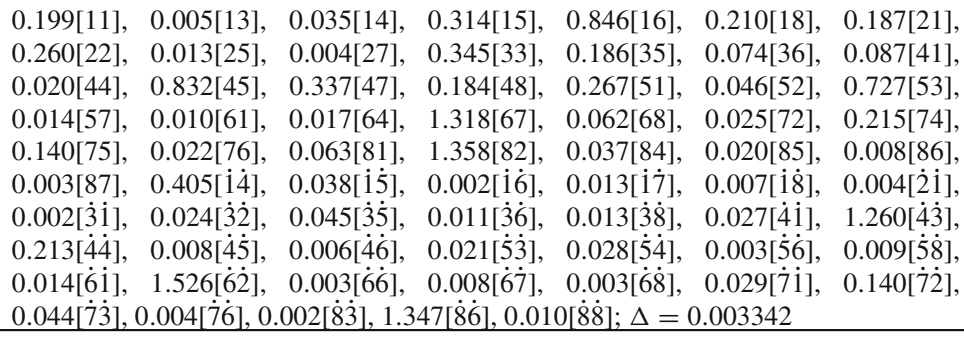 \\
\hline & $\begin{array}{l}-961.431,-871.387,-746.937,-578.759, \quad-578.444(\times 2), \quad-553.455, \\
-539.938(\times 2),-524.111,-418.566,-290.715(\times 2),-225.212,-149.827(\times 2), \\
-62.439(\times 2),-60.907,-44.332,-21.032,-10.641,-0.010(\times 2),-0.009(\times 2), \\
-0.008(\times 2),-0.007(\times 4),-0.006,-0.005,-0.004,-0.003(\times 2),-0.002(\times 7), \\
-0.001(\times 2), 0.000(\times 10), 0.001(\times 2), 0.002(\times 3), 0.003(\times 4), 0.004,0.005, \\
0.006,0.007(\times 2), 0.008(\times 4), 0.009(\times 2), 0.010,222.116(\times 2), 494.259(\times 2), \\
960.625,1005.441(\times 2), 1044.677,1227.541,1343.900,1409.361,1479.735, \\
1647.438(\times 2), 1747.482,1768.989(\times 2), 1858.271,1997.722(\times 2), 2128.839, \\
2381.293(\times 2), 2653.770,2702.885,2956.773(\times 2), 2973.527,3161.434,3448.716, \\
3463.422,4109.545(\times 2), 4246.831(\times 2), 4807.025(\times 2), 4862.019,4963.983, \\
5295.965,5307.813,5565.091,5630.780,7731.976,7735.146,8719.021,8726.270, \\
11977.576(\times 2), 12488.517,12490.001\end{array}$ \\
\hline $\mathcal{M}_{v} / g$ & $\begin{array}{l}-93.273,-93.270,-75.926,-75.715,-67.671(\times 2),-56.845,- \\
-53.525,-53.246(\times 2),-51.081(\times 2),-49.351,-49.246,-47.662,- \\
-38.342,-38.066,-37.589(\times 2),-37.200,-34.630(\times 2),-11.790,-5.75 \\
0.000(\times 74), 5.751(\times 2), 11.790,34.630(\times 2), 37.200,37.589(\times 2), 38.066, \\
43.587,47.662,49.246,49.351,51.081(\times 2), 53.246(\times 2), 53.525,53.847, \\
67.671(\times 2), 75.715,75.926,93.270,93.273\end{array}$ \\
\hline$A_{1}$ & $\begin{array}{l}-52.999(\times 2),-49.817(\times 2),-36.317(\times 2),-24.985(\times 2), 24.985(\times 2), 36.317(\times 2), \\
49.817(\times 2), 52.999(\times 2)\end{array}$ \\
\hline$A_{3}$ & 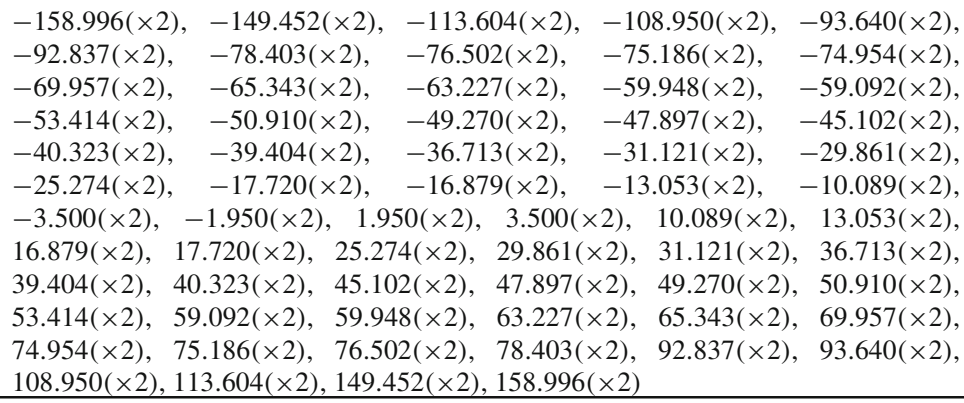 \\
\hline
\end{tabular}

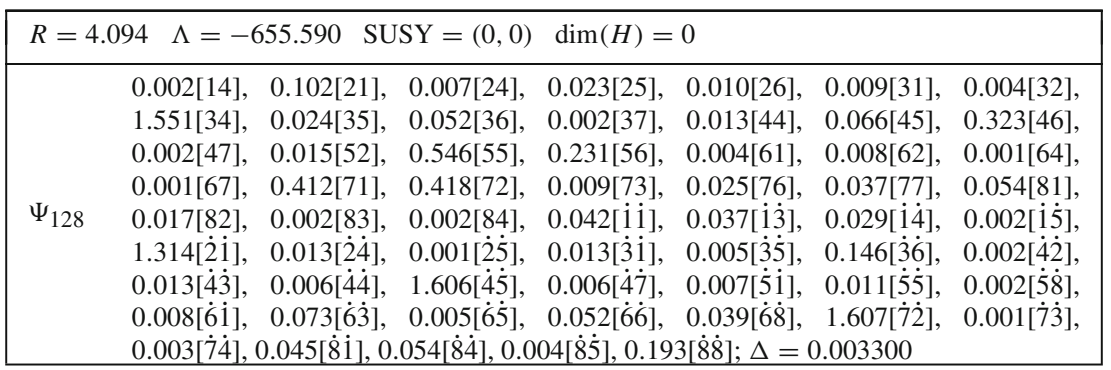




\begin{tabular}{|c|c|}
\hline $\mathcal{M}_{s} / g^{2}$ & $\begin{array}{l}-1012.029,-811.917,-751.597,-714.762,-688.136,-620.133,-582.146, \\
-553.942,-410.439,-253.240,-235.093,-198.936,-81.152,-79.451,-77.278, \\
-52.855,-38.525,-26.903,-11.180,-0.008(\times 2),-0.007(\times 2),-0.006(\times 2), \\
-0.005(\times 2),-0.004(\times 3),-0.003(\times 3),-0.002(\times 4),-0.001(\times 3), 0.000(\times 10), \\
0.001(\times 2), 0.002(\times 4), 0.003(\times 3), 0.004(\times 4), 0.005(\times 3), 0.006,0.007(\times 3), \\
0.008(\times 2), 0.009,0.010(\times 2), 137.201,329.714,353.226,400.062,513.021,653.957, \\
703.543,864.745,1123.432,1124.993,1127.707,1234.097,1241.335,1267.587, \\
1355.796,1460.168,1659.805,1935.738,2011.002,2044.990,2340.362,2349.061, \\
2360.750,2563.703,2629.901,2684.135,2711.330,3053.953,3159.414,3235.807, \\
3303.989,3350.331,3404.991,3792.567,3807.527,4862.031,4942.744,5243.993, \\
5272.802,5273.878,5276.263,5503.898,5504.532,6147.373,6179.919,7926.966, \\
7929.220,9095.116,9100.172,12545.088,12546.401,14217.741,14217.743\end{array}$ \\
\hline $\mathcal{M}_{v} / g$ & $\begin{array}{l}-94.757,-94.756,-76.332,-76.184,-74.948,-74.941,-59.751,-59.398, \\
-57.669,-55.987,-53.422,-50.117,-49.336,-49.073,-48.572,-48.294, \\
-43.644,-40.588,-39.491,-39.201,-38.809,-38.005,-33.338,-32.209, \\
-9.934,-6.091,-4.876,-2.001,0.000(\times 72), 2.001,4.876,6.091,9.934,32.209, \\
33.338,38.005,38.809,39.201,39.491,40.588,43.643,48.294,48.572,49.073, \\
49.336,50.117,53.422,55.987,57.669,59.398,59.751,74.941,74.948,76.184, \\
76.332,94.756,94.757\end{array}$ \\
\hline$A_{1}$ & $\begin{array}{l}-53.284(\times 2),-50.947(\times 2),-38.306(\times 2),-27.065(\times 2), 27.065(\times 2), 38.306(\times 2), \\
50.947(\times 2), 53.284(\times 2)\end{array}$ \\
\hline$A_{3}$ & 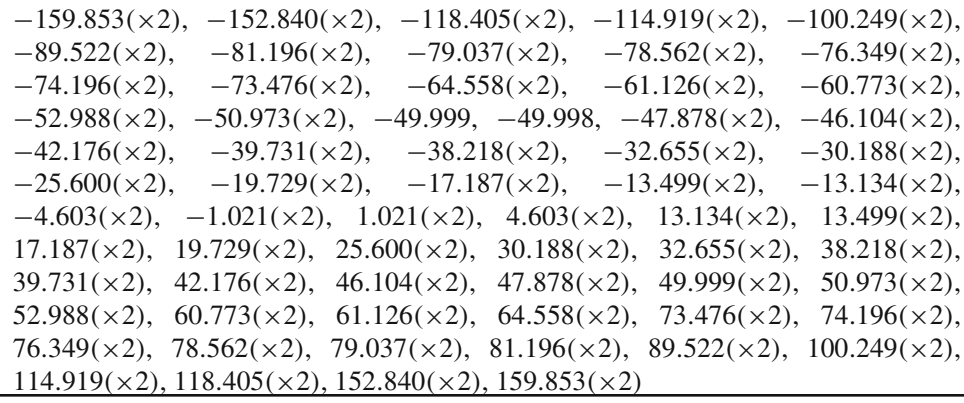 \\
\hline
\end{tabular}

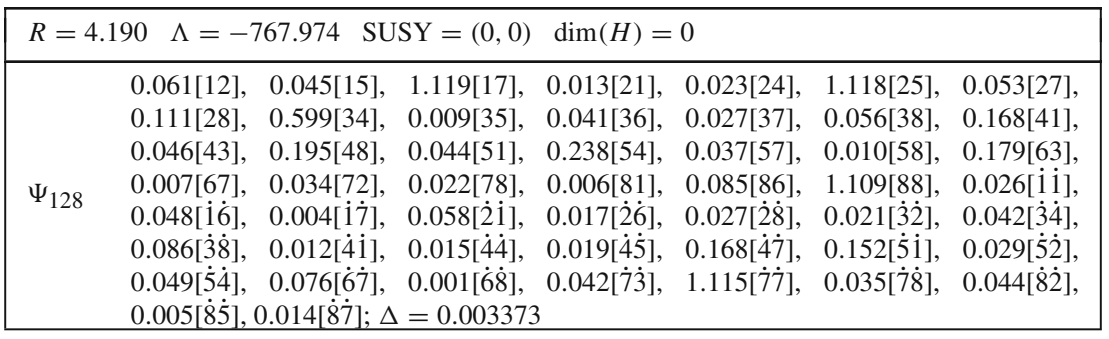




\begin{tabular}{|c|c|}
\hline $\mathcal{M}_{s} / g^{2}$ & $\begin{array}{l}-1103.825,-935.198,-861.555,-822.745,-783.843,-728.998,-653.693, \\
-599.926,-556.618,-484.634,-420.054,-398.829,-307.750,-292.462, \\
-242.969,-156.829,-127.890,-86.310,-71.771,-4.838,-0.007,-0.006(\times 2), \\
-0.005(\times 4),-0.004(\times 2),-0.003(\times 4),-0.002(\times 7),-0.001(\times 3), 0.000(\times 14), \\
0.001(\times 2), 0.002(\times 4), 0.003(\times 5), 0.004(\times 3), 0.005(\times 3), 0.006(\times 2), 51.500, \\
231.743,265.240,275.734,511.880,710.394,876.290,903.543,1120.304,1268.149, \\
1373.563,1449.280,1594.140,1666.665,1876.342,1950.026,2093.384,2154.383, \\
2294.735,2346.125,2498.590,2679.117,2757.265,2843.589,2925.700,3028.232, \\
3130.834,3167.516,3273.593,3300.196,3456.884,3538.072,3631.537,3667.341, \\
3752.928,3843.749,3949.638,4034.316,4343.478,4375.390,4423.369,4521.789, \\
4780.774,4825.814,5261.808,5302.704,5845.287,5869.706,6344.274,6381.751, \\
7978.583,7992.382\end{array}$ \\
\hline $\mathcal{M}_{v} / g$ & $\begin{array}{l}-75.999,-75.905,-69.522,-68.747,-65.931,-64.954,-61.552,-59.556, \\
-55.395,-54.860,-53.746,-53.450,-51.063,-50.440,-48.102,-46.443, \\
-45.173,-43.045,-41.886,-39.170,-39.007,-34.647,-30.706,-26.062, \\
-21.606,-11.679,-10.280,-4.508,0.000(\times 72), 4.427,11.174,13.113,21.739, \\
23.840,33.771,34.901,36.064,41.840,43.091,44.528,46.986,47.698,49.210, \\
50.700,51.938,53.538,53.899,54.328,55.355,57.851,58.853,61.499,62.599, \\
69.579,69.955,75.429,75.533\end{array}$ \\
\hline$A_{1}$ & $\begin{array}{l}-56.975,-56.552,-40.468,-38.611,-37.498,-31.544,-31.170,-29.928, \\
27.933,32.438,33.655,37.222,40.094,41.594,54.369,55.442\end{array}$ \\
\hline$A_{3}$ & $\begin{array}{l}-170.926,-169.655,-121.405,-115.832,-112.493,-94.632,-93.510,-89.785, \\
-86.725,-86.684,-82.768,-82.532,-78.324,-78.223,-74.618,-74.279, \\
-72.412,-72.035,-69.992,-69.130,-66.850,-66.217,-64.068,-63.640, \\
-63.047,-61.384,-61.126,-60.089,-59.709,-58.796,-58.153,-57.501, \\
-56.526,-55.955,-54.345,-53.466,-52.698,-49.677,-48.258,-47.446, \\
-45.639,-45.072,-43.016,-42.187,-40.116,-36.127,-33.578,-30.182, \\
-28.744,-27.988,-26.045,-25.073,-24.685,-22.708,-20.324,-18.259, \\
-14.092,-12.518,-10.394,-9.369,-6.881,-5.233,-3.226,-1.550,-0.276, \\
2.373,4.232,6.415,8.801,10.735,15.123,16.395,17.239,20.086,21.094,25.061, \\
27.237,28.245,29.200,29.937,31.230,34.621,36.536,39.097,40.866,43.432, \\
44.673,46.601,48.753,49.602,50.380,52.142,52.619,53.916,55.295,56.593, \\
57.861,58.175,59.436,59.963,60.068,60.205,61.203,63.284,64.081,65.427, \\
65.583,66.145,68.693,68.833,70.892,71.506,73.651,74.103,76.741,76.833, \\
81.112,81.277,83.798,88.177,88.181,97.314,100.966,111.666,120.281,124.781, \\
163.108,166.325\end{array}$ \\
\hline
\end{tabular}

\begin{tabular}{|c|c|c|c|c|}
\hline \multicolumn{5}{|c|}{$R=4.309 \quad \Lambda=-911.543 \quad \operatorname{SUSY}=(0,0) \quad \operatorname{dim}(H)=0$} \\
\hline$\Psi_{128}$ & 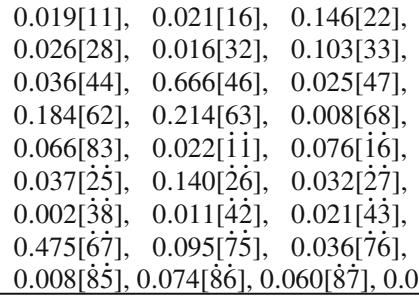 & $\begin{array}{ll}0.004[23], & 0.034[24], \\
0.076[34], & 0.030[36], \\
0.035[48], & 0.141[51], \\
0.093[72], & 0.073[75], \\
0.001[\dot{1}]], & 0.023[\dot{2} \dot{1}], \\
0.508[\dot{3} \dot{1}], & 0.573[\dot{3} \dot{3}], \\
0.096[\dot{4} \dot{4}], & 0.059[\dot{5} \dot{1}], \\
0.022[\dot{7} \dot{8}], & 0.023[\dot{8} \dot{1}], \\
93 \dot{\dot{8}} \dot{\dot{8}}] ; \Delta=0.003268\end{array}$ & $\begin{array}{l}0.145[26], \\
0.114[41], \\
0.040[55], \\
0.070[77], \\
0.047[\dot{2} \dot{2}], \\
0.096[\dot{3} \dot{6}], \\
0.129[\dot{5} \dot{2}], \\
1.444[\dot{8} \dot{2}],\end{array}$ & $\begin{array}{l}0.029 \text { [27], } \\
0.016[43], \\
0.084[57], \\
1.050[82], \\
0.525[\dot{2} \dot{3}], \\
0.019[\dot{3} \overline{7}], \\
0.496[\dot{6} \dot{6}], \\
0.017[\dot{8} \dot{4}],\end{array}$ \\
\hline
\end{tabular}




\begin{tabular}{|c|c|}
\hline $\mathcal{M}_{s} / g^{2}$ & $\begin{array}{l}-1150.080,-1121.661,-1055.848,-1004.104,-938.075,-787.238,-713.579, \\
-669.609,-553.961,-553.242,-459.192,-354.283,-342.803,-311.433, \\
-215.717,-83.764,-76.673,-0.010,-0.009,-0.008(\times 3),-0.007,-0.006(\times 3), \\
-0.005(\times 3),-0.004(\times 2),-0.003(\times 5),-0.002(\times 5),-0.001(\times 2), 0.000(\times 7), \\
0.001(\times 3), 0.002(\times 4), 0.003(\times 3), 0.004(\times 4), 0.005(\times 3), 0.006,0.007(\times 2), \\
0.008(\times 2), 0.009,54.066,88.685,167.596,306.962,594.153,760.377,790.911, \\
892.197,1182.776,1366.438,1583.702,1802.421,1941.253,2093.611,2137.310, \\
2337.650,2515.377,2516.887,2688.795,2732.827,3117.055,3204.136,3348.059, \\
3476.275,3504.856,3965.142,4031.366,4203.825,4253.643,4317.650,4368.742, \\
4431.327,4570.893,4895.913,4973.603,5207.302,5254.741,5588.250,5609.380, \\
5841.322,5860.775,6472.300,6498.843,7171.182,7209.050,7480.986,7644.535, \\
8416.904,8417.428,8565.850,8599.895,10737.559,10749.021,13442.504, \\
13443.951\end{array}$ \\
\hline $\mathcal{M}_{v} / g$ & $\begin{array}{l}-95.964,-95.913,-83.101,-82.713,-77.937,-77.532,-72.164,-70.350, \\
-68.711,-66.085,-63.157,-60.463,-58.774,-57.782,-55.481,-52.961, \\
-52.150,-49.138,-48.697,-45.356,-45.011,-41.508,-37.829,-27.766, \\
-24.468,-13.506,-10.287,-4.676,0.000(\times 72), 4.677,10.287,13.506,24.468, \\
27.766,37.828,41.508,45.011,45.356,48.697,49.138,52.150,52.961,55.481, \\
57.782,58.774,60.463,63.157,66.085,68.711,70.350,72.164,77.532,77.937, \\
82.713,83.101,95.913,95.964\end{array}$ \\
\hline$A_{1}$ & $\begin{array}{l}-68.139,-67.782,-49.553,-46.019,-40.295,-38.613,-33.757,-32.562, \\
32.562,33.757,38.613,40.295,46.019,49.553,67.782,68.139\end{array}$ \\
\hline$A_{3}$ & $\begin{array}{l}-204.416,-203.346,-148.659,-138.058,-120.884,-115.840,-110.737, \\
-110.732,-104.019,-104.011,-101.271,-97.685,-94.776,-94.616,-93.737, \\
-93.702,-90.056,-89.324,-81.244,-80.537,-77.428,-76.039,-75.688, \\
-74.506,-72.671,-72.219,-71.249,-69.976,-69.689,-67.677,-66.101, \\
-65.895,-64.903,-63.531,-63.194,-62.568,-61.318,-60.944,-59.752, \\
-54.806,-54.234,-52.036,-50.396,-44.704,-43.251,-42.498,-41.047, \\
-40.032,-39.260,-35.594,-33.455,-31.138,-29.796,-28.039,-25.282, \\
-20.434,-18.186,-16.778,-16.472,-14.188,-12.631,-5.681,-3.557,-2.097, \\
2.097,3.557,5.681,12.631,14.188,16.472,16.778,18.186,20.434,25.282,28.039, \\
29.796,31.138,33.455,35.594,39.260,40.032,41.047,42.498,43.251,44.704, \\
50.396,52.036,54.234,54.806,59.752,60.944,61.318,62.568,63.194,63.531, \\
64.903,65.895,66.101,67.677,69.689,69.976,71.249,72.219,72.671,74.506, \\
75.688,76.039,77.428,80.537,81.244,89.324,90.056,93.702,93.737,94.616, \\
94.776,97.685,101.271,104.011,104.019,110.732,110.737,115.840,120.884, \\
138.057,148.659,203.346,204.416\end{array}$ \\
\hline
\end{tabular}

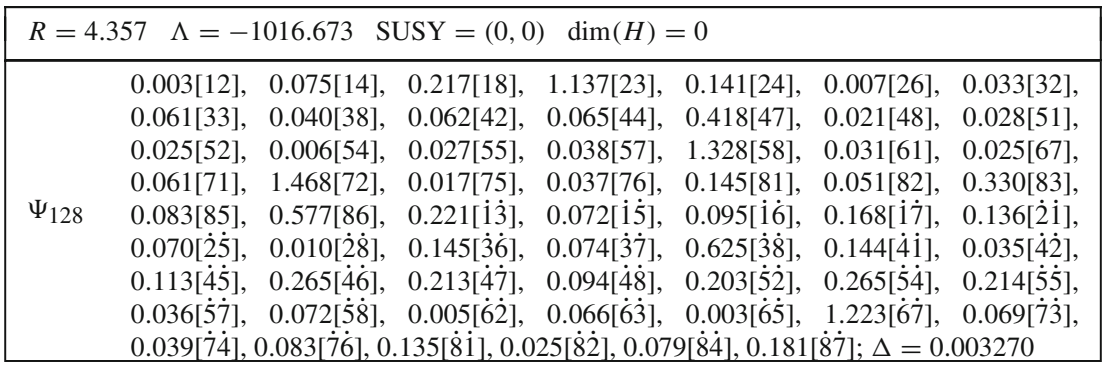




\begin{tabular}{|c|c|}
\hline $\mathcal{M}_{s} / g^{2}$ & $\begin{array}{l}-1278.931,-1118.109,-1028.346,-1004.185,-855.757,-829.222,-659.388, \\
-633.003,-565.663,-492.818,-443.892,-386.841,-307.453,-291.322, \\
-243.742,-136.731,-114.169,-103.356,-0.011,-0.010,-0.009,-0.008(\times 3), \\
-0.007(\times 2),-0.006(\times 2),-0.005(\times 2),-0.004(\times 4),-0.003(\times 3),-0.002(\times 5), \\
-0.001,0.000(\times 7), 0.001,0.002(\times 3), 0.003(\times 3), 0.004(\times 4), 0.005(\times 2), 0.006(\times 2), \\
0.007(\times 3), 0.008,0.009(\times 2), 0.010(\times 2), 0.011,92.943,278.179,407.567,612.943, \\
671.104,874.225,1089.564,1219.937,1550.751,1777.927,1947.704,2043.444, \\
2100.614,2349.021,2418.698,2718.442,2919.001,3038.218,3177.323,3277.896, \\
3391.399,3510.092,3591.201,3667.987,3810.694,4006.837,4239.848,4353.782, \\
4421.520,4501.889,4621.516,4835.248,4865.787,5027.882,5181.017,5297.545, \\
5464.629,5598.631,5727.093,5735.181,6209.359,6352.628,6612.913,6714.923, \\
6938.763,7141.846,7776.374,7910.708,8560.921,8596.960,9313.899,9337.986, \\
11147.532,11154.964\end{array}$ \\
\hline $\mathcal{M}_{v} / g$ & $\begin{array}{l}-92.647,-92.462,-82.128,-81.128,-76.930,-74.668,-71.549,-68.913, \\
-66.122,-62.674,-61.887,-61.346,-60.336,-59.544,-57.878,-56.936, \\
-55.481,-52.122,-51.906,-50.020,-47.829,-41.521,-39.983,-38.813, \\
-33.063,-27.693,-15.309,-7.935,0.000(\times 72), 6.520,17.871,26.585,32.938, \\
35.080,40.657,44.618,48.816,49.948,52.931,54.460,55.148,56.912,58.834, \\
60.178,61.343,61.719,63.762,65.636,67.225,70.586,72.417,75.264,76.197, \\
78.523,79.630,87.430,87.596\end{array}$ \\
\hline$A_{1}$ & $\begin{array}{l}-63.847,-62.295,-53.283,-48.368,-45.450,-43.410,-41.766,-30.110, \\
33.369,38.925,44.904,46.656,53.194,54.929,58.020,58.533\end{array}$ \\
\hline$A_{3}$ & $\begin{array}{l}-191.540,-186.886,-159.849,-145.103,-136.351,-130.229,-125.298, \\
-108.212,-108.181,-98.155,-98.025,-90.329,-88.505,-88.394,-87.332, \\
-87.068,-83.649,-82.964,-82.846,-81.407,-81.037,-78.585,-78.105, \\
-77.780,-76.887,-75.918,-73.950,-73.458,-72.887,-70.855,-70.212, \\
-69.627,-68.584,-65.967,-65.165,-63.233,-63.161,-60.706,-58.371, \\
-56.235,-53.724,-51.946,-49.995,-49.149,-47.894,-46.218,-43.491, \\
-43.421,-41.692,-41.026,-36.208,-34.572,-33.642,-31.630,-30.157, \\
-22.290,-20.702,-18.638,-16.693,-13.697,-12.290,-8.825,-3.914,-1.001, \\
3.285,4.953,7.604,10.254,13.894,14.793,19.752,23.020,24.031,28.575,30.462, \\
33.050,35.233,37.595,38.510,40.400,43.529,45.782,47.432,47.857,48.486, \\
50.661,54.740,55.942,56.775,57.757,58.962,62.643,64.390,65.533,66.714, \\
67.344,68.408,69.975,70.709,71.490,72.841,74.412,75.208,76.885,77.322, \\
78.360,80.255,81.322,81.771,82.439,85.948,86.323,87.038,88.054,89.892, \\
91.502,96.609,96.886,100.106,102.292,102.371,116.774,134.711,139.968, \\
159.583,164.786,174.059,175.598\end{array}$ \\
\hline
\end{tabular}

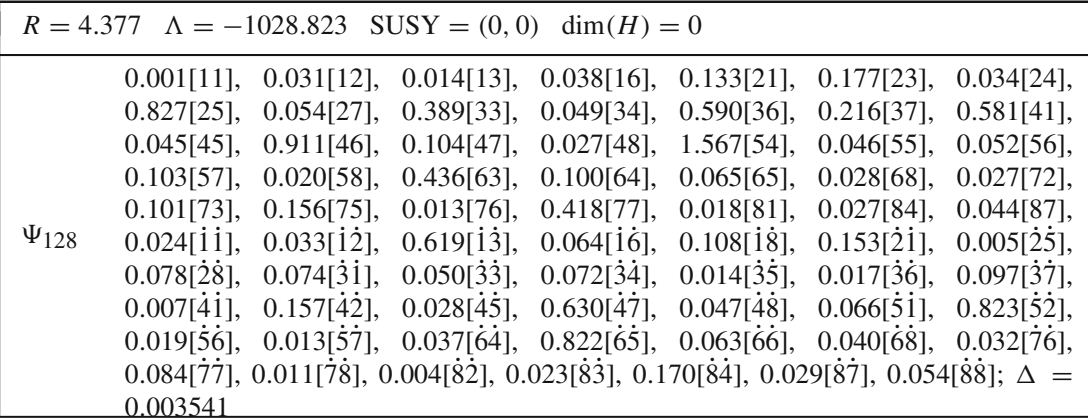




\begin{tabular}{|c|c|}
\hline $\mathcal{M}_{s} / g^{2}$ & $\begin{array}{l}-1478.709,-1419.319,-1170.348,-967.967,-908.253,-868.791,-736.002, \\
-683.904,-483.083,-480.318,-438.540,-399.539,-192.961,-110.100, \\
-63.015,-0.010,-0.009(\times 2),-0.008(\times 2),-0.007(\times 2),-0.006(\times 4), \\
-0.005(\times 2),-0.004(\times 4),-0.003(\times 3),-0.002(\times 4),-0.001(\times 2), 0.000(\times 8), \\
0.001(\times 2), 0.002(\times 4), 0.003(\times 4), 0.004(\times 3), 0.005(\times 2), 0.006(\times 2), 0.007, \\
0.008(\times 2), 0.009(\times 2), 73.944,81.365,395.500,413.369,609.662,910.467, \\
1069.908,1247.410,1484.631,1484.900,1602.183,1648.599,1797.437,1856.811, \\
1931.025,1933.417,2334.015,2351.773,2660.162,2678.769,2718.576,2998.046, \\
3202.138,3264.332,3294.202,3411.055,3943.157,4020.324,4268.784,4282.312, \\
4497.938,4587.508,4686.620,4694.509,4843.972,4859.892,5028.800,5098.268, \\
5296.179,5316.868,5335.798,5383.102,5586.400,5887.699,5919.077,6169.445, \\
6397.927,6475.187,6554.693,7036.747,7348.492,7392.040,7661.201,8011.419, \\
8374.832,9238.865,9411.959\end{array}$ \\
\hline $\mathcal{M}_{v} / g$ & $\begin{array}{l}-80.113,-79.623,-77.817,-76.304,-76.257,-73.996,-73.155,-70.466, \\
-70.153,-69.595,-67.073,-63.776,-62.127,-61.245,-60.278,-58.379, \\
-56.155,-54.329,-52.563,-50.983,-46.669,-43.088,-41.854,-35.009, \\
-32.674,-12.691,-9.655,-6.516,0.000(\times 72), 6.516,9.655,12.691,32.674, \\
35.009,41.854,43.088,46.669,50.983,52.563,54.329,56.155,58.379,60.278, \\
61.245,62.127,63.776,67.073,69.595,70.153,70.466,73.155,73.996,76.257, \\
76.304,77.816,79.623,80.113\end{array}$ \\
\hline$A_{1}$ & $\begin{array}{l}-62.588,-61.869,-50.044,-47.791,-44.996,-42.782,-40.245,-35.164, \\
35.164,40.245,42.782,44.996,47.791,50.044,61.869,62.588\end{array}$ \\
\hline$A_{3}$ & $\begin{array}{l}-187.765,-185.606,-150.131,-143.372,-134.989,-128.346,-120.735, \\
-105.492,-94.020,-93.485,-93.230,-92.935,-88.527,-87.849,-86.032, \\
-85.538,-84.380,-82.167,-81.192,-80.560,-79.057,-77.564,-76.660, \\
-74.590,-73.993,-73.844,-73.477,-72.801,-72.729,-71.584,-71.103, \\
-70.139,-69.718,-67.915,-67.674,-66.547,-65.631,-63.259,-61.809, \\
-60.756,-55.569,-54.266,-52.662,-52.475,-49.747,-49.146,-44.625, \\
-41.190,-37.112,-35.196,-34.855,-33.177,-31.676,-31.217,-30.675, \\
-29.882,-24.616,-22.389,-18.870,-17.386,-12.492,-10.517,-2.184, \\
-1.295,1.295,2.184,10.517,12.492,17.386,18.870,22.389,24.616,29.882,30.675, \\
31.217,31.676,33.177,34.855,35.196,37.112,41.190,44.625,49.146,49.747, \\
52.475,52.662,54.266,55.569,60.756,61.809,63.259,65.631,66.547,67.674, \\
67.915,69.718,70.139,71.103,71.584,72.729,72.801,73.477,73.844,73.993, \\
74.590,76.660,77.564,79.057,80.560,81.192,82.167,84.380,85.538,86.032, \\
87.849,88.527,92.935,93.230,93.485,94.020,105.492,120.735,128.346,134.989, \\
143.372,150.131,185.606,187.765\end{array}$ \\
\hline
\end{tabular}

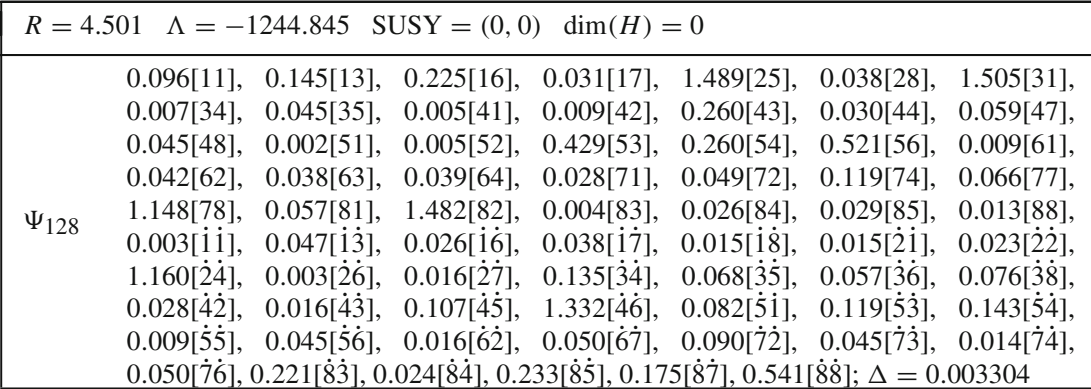




\begin{tabular}{|c|c|}
\hline $\mathcal{M}_{s} / g^{2}$ & $\begin{array}{l}-1645.366,-1432.959,-1373.115,-1266.871,-1121.244,-1092.501,-894.976, \\
-833.199,-676.370,-464.101,-399.464,-312.512,-206.323,-159.214, \\
-100.312,-22.881,-0.013,-0.011,-0.010,-0.009(\times 2),-0.008,-0.007(\times 3), \\
-0.006(\times 2),-0.005(\times 2),-0.004(\times 3),-0.003(\times 3),-0.002(\times 2),-0.001, \\
0.000(\times 7), 0.001,0.002(\times 2), 0.003(\times 4), 0.004(\times 2), 0.005(\times 3), 0.006(\times 2), \\
0.007(\times 3), 0.008(\times 2), 0.009(\times 2), 0.010(\times 2), 0.012,0.013(\times 2), 0.014,141.933, \\
421.134,490.565,869.735,1245.168,1282.327,1617.820,1787.288,2150.445, \\
2273.011,2543.701,2816.327,2902.355,2981.369,3381.536,3476.927,3709.728, \\
3894.949,4011.258,4104.620,4678.122,4722.985,5003.087,5170.149,5216.874, \\
5424.218,5548.590,5690.828,5753.846,5827.295,5994.846,6167.380,6200.031, \\
6415.535,6652.022,6705.925,6919.602,7238.908,7358.270,7554.756,7832.448, \\
8180.181,8329.611,8579.582,9082.928,9264.282,9768.573,9902.092,10830.925, \\
10911.787,11396.687,11419.829,12385.749,12408.334,17160.636,17166.949\end{array}$ \\
\hline $\mathcal{M}_{v} / g$ & $\begin{array}{l}-107.471,-107.292,-95.608,-95.424,-85.893,-84.958,-83.242,-81.444, \\
-79.316,-76.806,-73.434,-70.905,-69.256,-68.334,-66.637,-64.808, \\
-64.486,-61.141,-57.863,-56.637,-55.546,-53.711,-49.766,-46.561, \\
-40.269,-35.149,-16.617,-9.924,0.000(\times 72), 9.509,17.935,33.904,42.215, \\
47.951,49.640,54.111,56.405,57.080,57.697,61.291,63.121,64.874,66.452, \\
67.591,69.239,70.124,72.863,75.420,76.130,78.848,81.590,89.309,90.653, \\
92.890,93.907,108.748,109.002\end{array}$ \\
\hline$A_{1}$ & $\begin{array}{l}-73.869,-72.550,-62.138,-59.299,-51.061,-44.864,-43.207,-42.239, \\
38.362,43.452,49.549,50.227,58.072,63.801,71.765,74.002\end{array}$ \\
\hline$A_{3}$ & $\begin{array}{l}-221.608,-217.650,-186.415,-177.896,-153.184,-134.592,-129.622, \\
-126.718,-126.334,-126.313,-117.118,-117.083,-103.661,-103.410, \\
-101.766,-101.111,-98.877,-97.523,-95.445,-94.862,-91.247,-91.140, \\
-90.593,-89.404,-87.645,-87.185,-85.780,-84.809,-83.802,-82.193, \\
-81.737,-79.587,-79.109,-77.672,-76.143,-74.925,-73.924,-73.137, \\
-71.181,-69.563,-64.397,-62.476,-61.475,-59.541,-57.866,-56.880, \\
-53.231,-51.598,-50.167,-47.178,-44.502,-43.097,-40.145,-37.600, \\
-35.381,-31.915,-27.014,-25.025,-21.995,-17.266,-14.684,-9.107, \\
-5.540,-3.963,3.865,7.826,11.191,13.111,13.757,19.278,25.083,30.907,32.386, \\
33.840,38.860,40.972,43.633,46.018,49.304,50.951,53.704,56.004,57.582, \\
57.693,58.796,60.471,61.898,63.756,65.973,69.396,71.388,72.890,73.905, \\
75.772,76.648,77.622,79.166,80.928,81.897,83.638,85.271,86.319,87.242, \\
88.203,88.843,89.938,91.176,91.871,93.013,94.953,98.490,98.997,100.363, \\
101.504,104.477,104.606,115.086,120.344,120.381,125.091,125.134,130.355, \\
148.648,150.681,174.216,191.402,215.294,222.005\end{array}$ \\
\hline
\end{tabular}

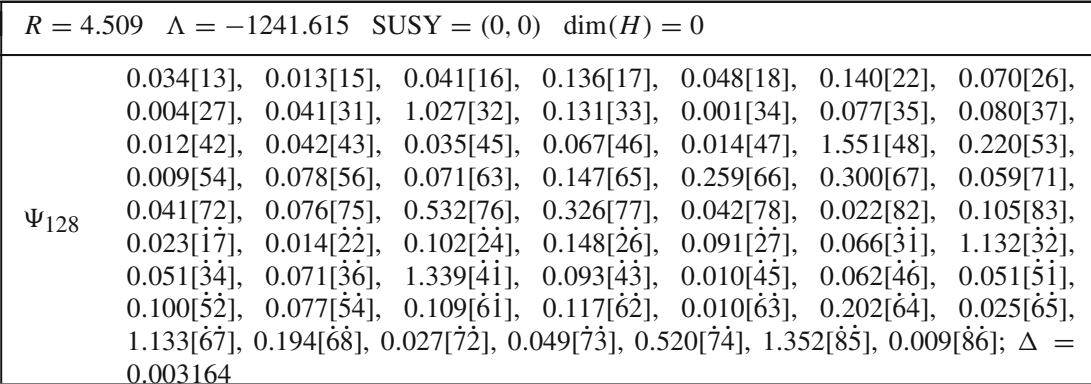




\begin{tabular}{|c|c|}
\hline $\mathcal{M}_{s} / g^{2}$ & $\begin{array}{l}-1578.932,-1385.689,-1318.880,-1176.835,-1069.835,-1022.769,-968.662, \\
-807.460,-571.011,-482.351,-458.444,-309.784,-224.587,-172.071, \\
-131.238,-72.773,-0.016,-0.015,-0.014,-0.012(\times 2),-0.011(\times 2),-0.010, \\
-0.009,-0.007(\times 3),-0.006(\times 4),-0.005(\times 2),-0.004(\times 2),-0.003(\times 3), \\
-0.002(\times 2), 0.000(\times 7), 0.002(\times 2), 0.003(\times 4), 0.004(\times 2), 0.005(\times 2), 0.006(\times 3), \\
0.007(\times 2), 0.008,0.009,0.010,0.011(\times 2), 0.013,0.014(\times 2), 0.016,254.938, \\
501.240,844.417,859.916,1030.473,1161.472,1291.671,1403.985,1730.555, \\
1767.571,1990.045,2731.371,2919.281,2932.727,3245.775,3532.303,3669.118, \\
3726.612,3771.252,4101.098,4176.104,4597.079,4696.941,4861.346,5123.485, \\
5194.785,5344.559,5841.197,5841.821,5939.504,6039.030,6465.767,6490.018, \\
6533.931,6580.181,6752.791,7334.119,7376.211,7907.594,7958.442,8776.908, \\
9037.882,9672.395,9798.191,9898.542,9929.927,10131.408,10322.214, \\
11378.585,11469.152,12529.156,12535.606,15675.876,15688.999,19041.792, \\
19041.911\end{array}$ \\
\hline $\mathcal{M}_{v} / g$ & $\begin{array}{l}-112.211,-112.145,-101.584,-101.457,-88.248,-87.039,-84.751,-83.667, \\
-81.065,-79.923,-73.982,-73.483,-70.989,-69.044,-68.067,-65.345, \\
-64.947,-61.870,-60.898,-57.436,-50.558,-49.763,-44.316,-43.745, \\
-28.172,-27.354,-22.664,-10.081,0.000(\times 72), 10.081,22.664,27.354,28.172, \\
43.745,44.316,49.763,50.558,57.436,60.898,61.870,64.947,65.345,68.067, \\
69.044,70.989,73.483,73.982,79.923,81.065,83.667,84.751,87.039,88.248, \\
101.457,101.584,112.145,112.211\end{array}$ \\
\hline$A_{1}$ & $\begin{array}{l}-77.546,-76.909,-61.618,-55.809,-53.952,-49.956,-39.369,-38.761, \\
38.761,39.369,49.956,53.952,55.809,61.618,76.909,77.546\end{array}$ \\
\hline$A_{3}$ & $\begin{array}{l}-232.637,-230.727,-184.855,-167.426,-161.857,-149.869,-134.610, \\
-134.604,-120.854,-120.799,-118.107,-116.284,-112.150,-112.006, \\
-108.466,-108.142,-102.389,-102.198,-100.450,-99.319,-94.467,-92.640, \\
-90.869,-89.984,-89.015,-86.750,-85.292,-84.602,-82.919,-81.758, \\
-79.838,-78.107,-76.579,-75.958,-75.168,-73.114,-72.247,-70.694, \\
-69.914,-69.431,-66.806,-64.754,-61.792,-61.197,-57.680,-54.945, \\
-53.130,-49.721,-48.902,-46.651,-43.043,-40.642,-39.403,-36.387, \\
-34.525,-33.020,-26.333,-23.357,-19.902,-11.988,-8.811,-7.358,-5.448, \\
-2.573,2.573,5.448,7.358,8.811,11.988,19.902,23.357,26.333,33.020,34.525, \\
36.387,39.403,40.642,43.043,46.651,48.902,49.721,53.130,54.945,57.680, \\
61.197,61.792,64.754,66.806,69.431,69.914,70.694,72.247,73.114,75.168, \\
75.958,76.579,78.107,79.838,81.758,82.919,84.602,85.292,86.750,89.015, \\
89.984,90.869,92.640,94.467,99.319,100.450,102.198,102.389,108.142,108.466, \\
112.006,112.150,116.284,118.107,120.799,120.854,134.604,134.610,149.869, \\
161.857,167.426,184.855,230.727,232.637\end{array}$ \\
\hline
\end{tabular}

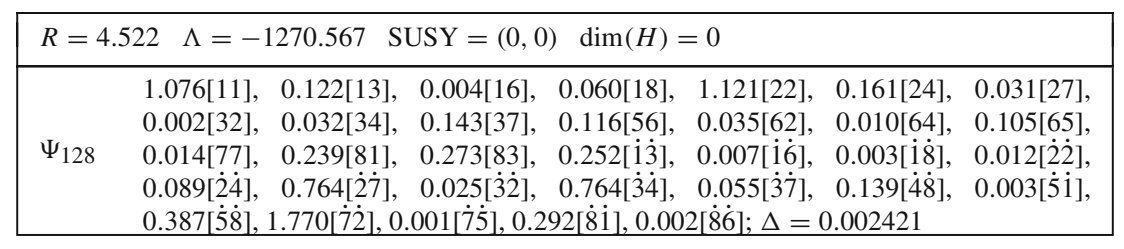




\begin{tabular}{|c|c|}
\hline $\mathcal{M}_{s} / g^{2}$ & $\begin{array}{l}-2260.063,-1992.853,-1231.194,-1189.783,-1094.496,-1065.413, \\
-1005.177,-826.712,-596.275,-589.453,-437.666,-393.416,-368.012, \\
-349.539,-163.281,-56.461,-28.492,-0.019,-0.017,-0.015(\times 2),-0.014, \\
-0.013,-0.012,-0.011(\times 2),-0.009(\times 2),-0.008,-0.007(\times 2),-0.006,-0.005, \\
-0.004(\times 2),-0.003(\times 3),-0.002(\times 2),-0.001,0.000(\times 6), 0.001,0.002(\times 2), \\
0.003(\times 3), 0.004(\times 2), 0.005(\times 2), 0.006,0.007(\times 2), 0.008,0.009(\times 2), 0.010(\times 2), \\
0.011,0.012,0.013,0.014(\times 2), 0.016,0.017,0.019,404.944,749.151,1182.798, \\
1200.236,1243.977,1353.436,1616.524,1927.284,2717.499,2906.258,3108.410, \\
3192.700,3353.634,3590.307,3593.199,3602.696,3725.898,4284.773,5476.794, \\
5570.422,5579.509,5663.427,5931.269,5950.938,6032.193,6064.956,6679.340, \\
6772.036,6961.262,7015.114,7070.624,7101.679,7306.426,7448.653,7982.813, \\
8048.917,8141.815,9263.774,9447.399,10213.640,10309.265,10793.603, \\
10870.250,11562.841,11599.136,13380.360,13381.978,13725.232,13769.900, \\
14871.418,14881.036,15462.515,15466.306,28952.750,28953.475\end{array}$ \\
\hline $\mathcal{M}_{v} / g$ & $\begin{array}{l}-124.563,-124.549,-108.278,-108.013,-96.744,-96.625,-90.362,-90.355, \\
-87.693,-86.901,-80.675,-76.073,-75.577,-70.766,-69.602,-69.210, \\
-64.227,-62.754,-60.155,-58.205,-56.741,-56.169,-52.313,-50.834, \\
-15.569,-14.377,-11.651,-7.948,0.000(\times 72), 7.948,11.651,14.377,15.569, \\
50.834,52.313,56.169,56.741,58.205,60.155,62.754,64.227,69.210,69.602, \\
70.766,75.577,76.073,80.675,86.901,87.693,90.355,90.362,96.625,96.744, \\
108.013,108.278,124.549,124.563\end{array}$ \\
\hline$A_{1}$ & $\begin{array}{l}-75.730,-75.201,-70.967,-68.455,-59.009,-51.137,-42.803,-42.353, \\
42.353,42.803,51.137,59.009,68.455,70.967,75.201,75.730\end{array}$ \\
\hline$A_{3}$ & $\begin{array}{l}-227.191,-225.604,-212.902,-205.366,-177.028,-153.410,-150.165, \\
-150.162,-145.291,-145.287,-131.111,-131.095,-128.409,-127.059, \\
-114.148,-114.110,-107.644,-107.294,-106.031,-105.965,-95.433, \\
-94.669,-94.476,-94.066,-91.384,-91.176,-88.072,-86.431,-82.927, \\
-80.754,-79.111,-78.477,-76.990,-75.784,-73.198,-73.065,-71.937, \\
-71.627,-68.620,-66.493,-66.392,-64.923,-64.389,-61.896,-61.413, \\
-61.172,-59.150,-58.873,-55.250,-55.180,-51.830,-47.338,-43.184, \\
-41.398,-38.030,-36.009,-31.581,-29.514,-29.114,-28.925,-22.940, \\
-12.176,-10.051,-7.550,7.550,10.051,12.176,22.940,28.925,29.114,29.514, \\
31.581,36.009,38.030,41.398,43.184,47.338,51.830,55.180,55.250,58.873, \\
59.150,61.172,61.413,61.896,64.389,64.923,66.392,66.493,68.620,71.627, \\
71.937,73.065,73.198,75.784,76.990,78.477,79.111,80.754,82.927,86.431, \\
88.072,91.176,91.384,94.066,94.476,94.669,95.433,105.965,106.031,107.294, \\
107.644,114.110,114.148,127.059,128.409,131.095,131.111,145.287,145.291, \\
150.162,150.165,153.410,177.028,205.366,212.902,225.604,227.191\end{array}$ \\
\hline
\end{tabular}

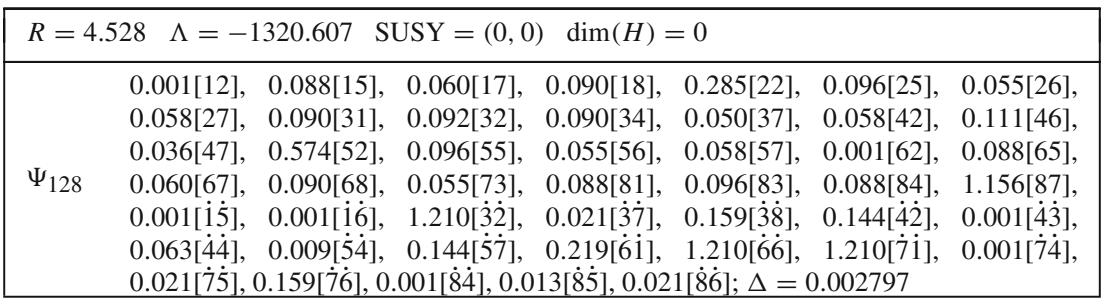




\begin{tabular}{|c|c|}
\hline $\mathcal{M}_{s} / g^{2}$ & $\begin{array}{l}-1898.123,-1776.021,-1689.302,-1462.590,-1418.215,-1108.474,-845.290, \\
-811.013,-694.666,-575.644,-345.224,-301.839,-243.644,-120.650, \\
-45.573,-0.011,-0.010,-0.009(\times 2),-0.008(\times 2),-0.007,-0.006(\times 3),-0.005, \\
-0.004(\times 2),-0.003(\times 5),-0.002(\times 3),-0.001,0.000(\times 6), 0.002(\times 5), 0.003(\times 3), \\
0.004(\times 2), 0.005(\times 3), 0.006(\times 2), 0.007(\times 3), 0.008(\times 2), 0.009(\times 3), 0.010, \\
0.011(\times 2), 0.013(\times 2), 69.634,73.499,234.847,496.500,1048.228,1198.888, \\
1373.109,1814.377,2136.241,2171.426,2613.012,2749.344,2994.676,3213.783, \\
3510.530,3541.315,3549.486,3872.594,3940.542,4216.167,4306.226,4911.142, \\
4921.008,5027.626,5068.757,5105.186,5282.387,5544.139,5633.177,5723.398, \\
5730.392,5753.639,5816.927,6172.878,6286.721,6368.725,6390.729,6397.609, \\
6733.791,6740.651,7062.010,7190.895,7225.350,7266.260,7603.339,7744.831, \\
7900.324,7943.116,8065.211,8404.509,8490.539,8544.765,8660.332,8887.673, \\
9284.014,11534.353,11593.147\end{array}$ \\
\hline $\mathcal{M}_{v} / g$ & $\begin{array}{l}-89.571,-89.457,-88.665,-86.479,-84.961,-82.816,-82.373,-81.453, \\
-79.258,-76.566,-74.968,-72.937,-72.396,-71.473,-67.684,-66.641, \\
-62.167,-60.799,-59.770,-57.376,-54.666,-52.657,-48.772,-46.293, \\
-42.367,-22.900,-22.148,-3.198,0.000(\times 72), 3.198,22.148,22.900,42.367, \\
46.293,48.772,52.657,54.666,57.376,59.770,60.799,62.167,66.641,67.684, \\
71.473,72.396,72.937,74.968,76.566,79.258,81.453,82.373,82.816,84.961, \\
86.479,88.665,89.457,89.571\end{array}$ \\
\hline$A_{1}$ & $\begin{array}{l}-68.170(\times 2),-62.453(\times 2),-45.314(\times 2),-43.494(\times 2), 43.494(\times 2), 45.314(\times 2), \\
62.453(\times 2), 68.170,68.171\end{array}$ \\
\hline$A_{3}$ & 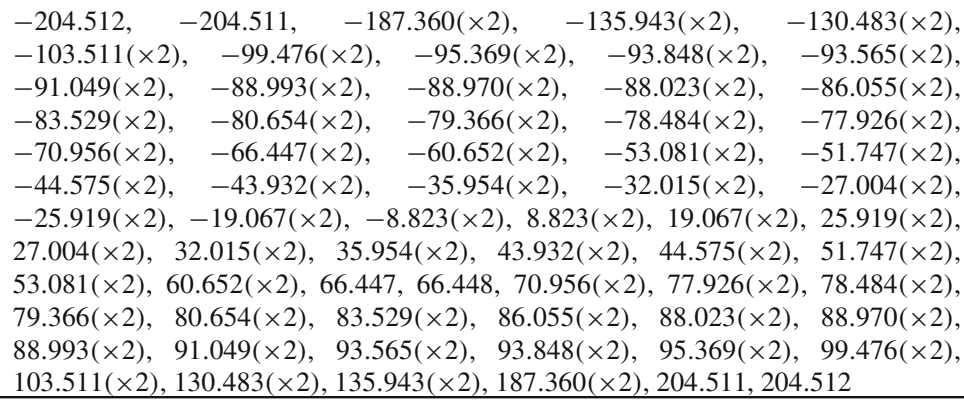 \\
\hline
\end{tabular}

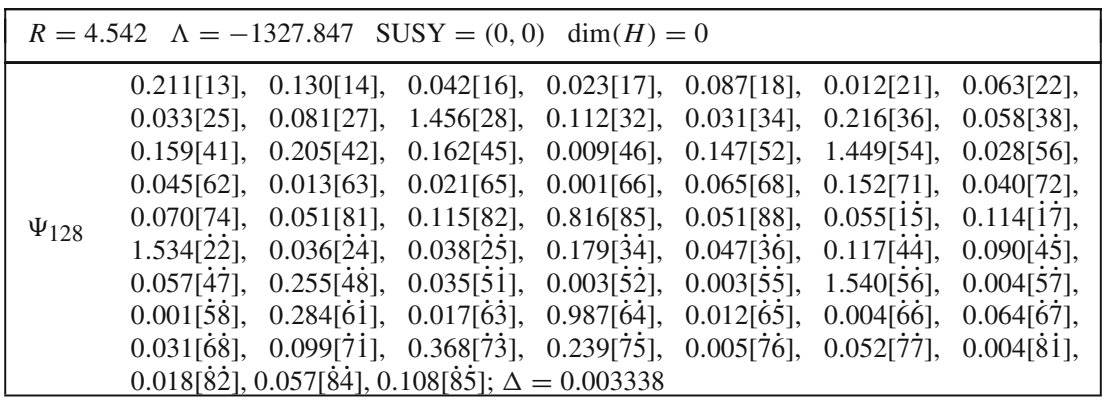




\begin{tabular}{|c|c|}
\hline $\mathcal{M}_{s} / g^{2}$ & $\begin{array}{l}-1785.103,-1643.027,-1528.136,-1168.456,-1119.242,-910.116,-712.002, \\
-617.736,-526.589,-483.293,-412.309,-213.710,-180.563,-88.291, \\
-86.879,-0.021(\times 2),-0.019,-0.018,-0.017,-0.016,-0.015,-0.014(\times 2), \\
-0.013,-0.012,-0.011,-0.010(\times 2),-0.009(\times 2),-0.008,-0.007(\times 2), \\
-0.005(\times 3),-0.004(\times 2),-0.003,-0.002(\times 3), 0.000(\times 5), 0.001,0.002(\times 2), \\
0.003(\times 2), 0.004,0.005(\times 2), 0.006(\times 2), 0.007,0.008(\times 4), 0.010,0.011,0.012(\times 3), \\
0.014,0.015,0.018,219.315,414.690,592.917,882.767,1100.062,1122.555, \\
1565.038,1691.037,1920.840,2159.499,2275.658,2502.505,2804.761,3500.584, \\
3511.083,3598.788,3797.236,3964.809,4036.204,4259.108,4450.089,4514.578, \\
5078.833,5134.795,5492.672,5604.631,5627.084,5746.086,5878.058,6108.314, \\
6275.686,6433.567,6444.891,6594.489,6828.424,6956.049,7042.247,7100.451, \\
7549.476,8029.638,8078.885,8755.384,8772.018,8966.907,9092.178,9887.617, \\
9935.581,10704.496,10710.542,12331.338,12347.107,12809.554,12835.552, \\
16687.718,16688.525,18816.425,18825.154\end{array}$ \\
\hline $\mathcal{M}_{v} / g$ & $\begin{array}{l}-114.378,-114.319,-96.119,-95.161,-91.782,-91.087,-87.562,-86.788, \\
-82.395,-80.725,-78.198,-77.714,-75.056,-70.476,-69.055,-67.426, \\
-63.119,-62.803,-62.011,-61.461,-57.226,-56.560,-49.763,-41.174, \\
-38.206,-36.656,-26.803,-7.386,0.000(\times 72), 7.386,26.803,36.656,38.206, \\
41.173,49.763,56.560,57.226,61.461,62.011,62.803,63.119,67.426,69.055, \\
70.476,75.056,77.714,78.198,80.725,82.395,86.788,87.562,91.087,91.782, \\
95.161,96.119,114.319,114.378\end{array}$ \\
\hline$A_{1}$ & $\begin{array}{l}-72.780,-70.839,-69.128,-66.472,-55.038,-50.600,-47.100,-38.241, \\
38.241,47.100,50.599,55.038,66.472,69.128,70.839,72.780\end{array}$ \\
\hline$A_{3}$ & $\begin{array}{l}-218.340,-212.516,-207.383,-199.416,-165.114,-151.799,-141.301, \\
-137.166,-137.147,-121.547,-121.477,-114.722,-112.921,-112.810, \\
-106.807,-106.462,-101.770,-101.159,-99.197,-98.311,-97.751,-97.027, \\
-92.054,-91.190,-89.861,-89.270,-88.076,-87.239,-85.473,-84.899, \\
-83.625,-82.465,-81.705,-81.210,-78.242,-76.951,-76.383,-74.940, \\
-74.547,-73.237,-69.435,-68.054,-65.030,-63.606,-62.367,-57.303, \\
-55.255,-54.322,-51.723,-48.004,-47.509,-45.706,-41.332,-37.509, \\
-34.935,-32.719,-30.066,-23.497,-22.623,-20.722,-14.844,-10.389, \\
-3.295,-2.398,2.398,3.295,10.389,14.844,20.722,22.623,23.497,30.067, \\
32.719,34.935,37.509,41.332,45.706,47.509,48.004,51.723,54.322,55.255, \\
57.303,62.367,63.606,65.030,68.054,69.435,73.237,74.547,74.940,76.383, \\
76.951,78.242,81.210,81.705,82.465,83.625,84.899,85.473,87.239,88.076, \\
89.270,89.861,91.190,92.054,97.027,97.751,98.311,99.197,101.159,101.771, \\
106.462,106.807,112.810,112.921,114.722,121.477,121.547,137.147,137.166, \\
141.301,151.798,165.114,199.416,207.383,212.516,218.341\end{array}$ \\
\hline
\end{tabular}

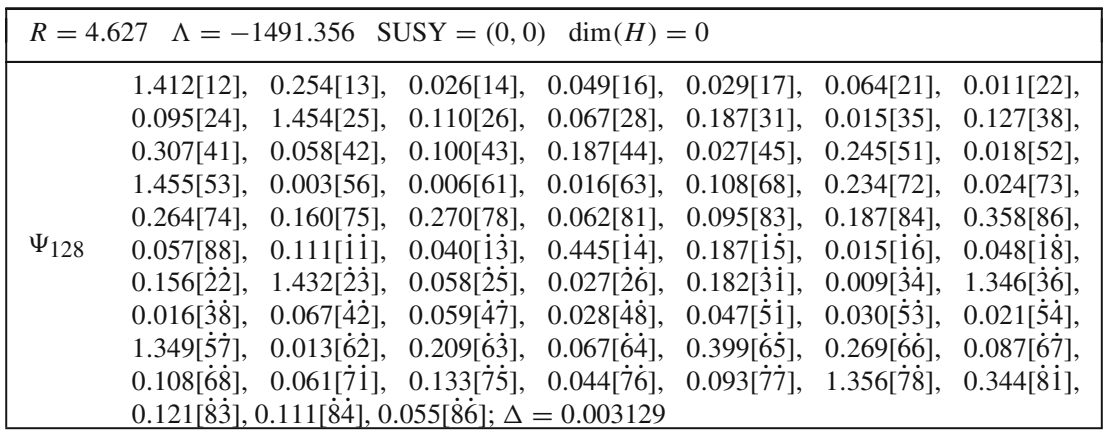




\begin{tabular}{|c|c|}
\hline $\mathcal{M}_{s} / g^{2}$ & $\begin{array}{l}-2165.484,-1802.100,-1642.451,-1435.729,-1400.430,-1178.314,-921.725, \\
-607.536,-335.168,-289.761,-272.077,-119.917,-70.388,-24.690,-0.032, \\
-0.029,-0.028,-0.025,-0.024,-0.023,-0.022,-0.021,-0.019,-0.017, \\
-0.016,-0.015,-0.013(\times 2),-0.012,-0.011(\times 2),-0.010,-0.009,-0.007(\times 2), \\
-0.005(\times 3),-0.004,-0.003,-0.001(\times 2), 0.000(\times 3), 0.002(\times 2), 0.003(\times 2), \\
0.004,0.005,0.006,0.007(\times 3), 0.008(\times 2), 0.010(\times 2), 0.012,0.013,0.015(\times 2), \\
0.016,0.018,0.020(\times 2), 0.022,0.024,0.026,486.597,764.680,1492.089,1672.880, \\
1822.161,2118.871,2332.170,2428.999,2637.630,2789.976,3072.809,3552.409, \\
3624.753,4169.770,4547.637,4715.392,4810.784,4966.994,5133.659,5469.224, \\
5907.130,6259.779,6494.919,6759.484,6968.989,7183.835,7497.699,7714.887, \\
7774.509,8005.504,8059.530,8411.817,8583.701,8839.486,9336.379,9361.785, \\
10168.775,10220.894,10643.617,10802.470,11438.488,11456.180,13285.777, \\
13291.011,14475.051,14629.558,15241.256,15477.703,16875.335,16884.956, \\
19703.671,19705.743,21490.494,21495.467,26723.154,26724.775,29770.400, \\
29771.084\end{array}$ \\
\hline $\mathcal{M}_{v} / g$ & $\begin{array}{l}-143.778,-143.761,-119.616,-119.577,-109.160,-108.803,-104.687, \\
-103.674,-97.675,-96.385,-88.054,-86.732,-82.827,-81.605,-77.630, \\
-75.465,-75.111,-70.627,-68.718,-67.587,-63.235,-62.381,-57.766, \\
-56.528,-51.639,-39.552,-20.099,-8.588,0.000(\times 72), 8.588,20.099,39.552, \\
51.639,56.528,57.766,62.381,63.235,67.587,68.718,70.627,75.111,75.465, \\
77.630,81.604,82.827,86.732,88.054,96.385,97.675,103.674,104.687,108.803, \\
109.160,119.577,119.616,143.761,143.778\end{array}$ \\
\hline$A_{1}$ & $\begin{array}{l}-90.995,-90.502,-76.472,-75.854,-59.027,-54.730,-52.716,-47.640, \\
47.640,52.716,54.730,59.027,75.854,76.472,90.502,90.995\end{array}$ \\
\hline$A_{3}$ & $\begin{array}{l}-272.984,-271.506,-229.416,-227.562,-177.082,-170.980(\times 2),-164.190, \\
-158.148,-152.883,-152.872,-144.101,-144.084,-142.921,-130.027, \\
-129.925,-125.045,-124.829,-122.235,-122.198,-114.024,-113.028, \\
-108.315,-106.894,-106.238,-104.910,-103.265,-102.867,-97.022, \\
-96.611,-95.189,-94.655,-92.999,-91.922,-90.443,-88.467,-87.194, \\
-85.922,-84.329,-82.281,-81.492,-79.999,-78.302,-73.075,-70.718, \\
-70.350,-66.770,-64.725,-57.269,-53.910,-52.258,-48.167,-47.221, \\
-44.610,-42.117,-38.805,-33.682,-31.063,-26.849,-21.784,-20.613, \\
-16.868,-10.921,-4.408,4.408,10.921,16.868,20.613,21.784,26.849,31.063, \\
33.682,38.805,42.117,44.610,47.221,48.167,52.258,53.910,57.269,64.725, \\
66.770,70.350,70.718,73.076,78.302,79.999,81.492,82.281,84.329,85.922, \\
87.194,88.467,90.443,91.922,92.999,94.655,95.189,96.611,97.022,102.867, \\
103.265,104.910,106.238,106.894,108.315,113.028,114.024,122.198,122.235, \\
124.829,125.045,129.925,130.027,142.921,144.084,144.101,152.872,152.883, \\
158.148,164.190,170.980(\times 2), 177.082,227.562,229.416,271.506,272.984\end{array}$ \\
\hline
\end{tabular}

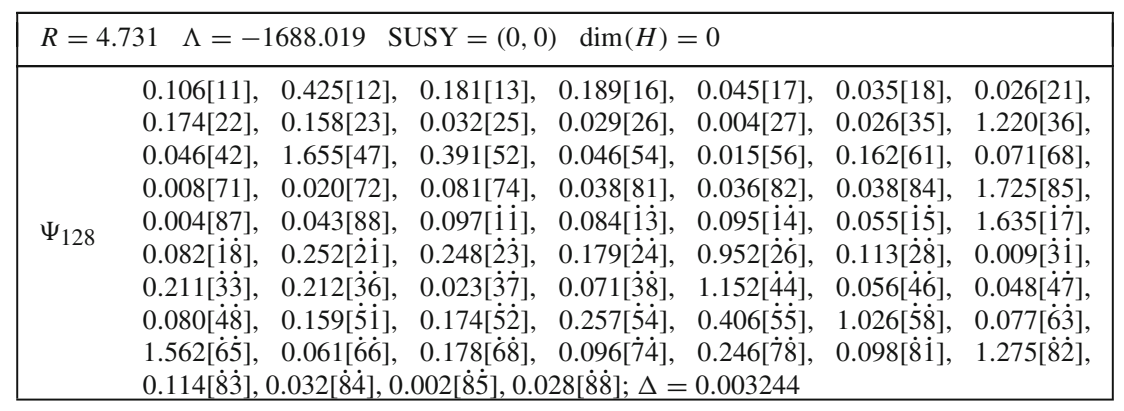




\begin{tabular}{|c|c|}
\hline $\mathcal{M}_{s} / g^{2}$ & $\begin{array}{l}-2100.387,-1855.354,-1799.434,-1510.064,-1390.345,-1097.218, \\
-1037.941,-843.416,-671.463,-632.664,-467.362,-137.672,-87.435, \\
-65.292,-0.061,-0.058,-0.056,-0.054,-0.053,-0.052,-0.049,-0.048, \\
-0.046(\times 2),-0.042,-0.041,-0.011,-0.007(\times 2),-0.006,-0.005(\times 2), \\
-0.004(\times 2),-0.003,-0.002,-0.001,0.000(\times 4), 0.001,0.002,0.003(\times 3), \\
0.004(\times 2), 0.005,0.006(\times 2), 0.008(\times 2), 0.009(\times 2), 0.011,0.012,0.016,0.043, \\
0.046(\times 2), 0.050,0.051,0.055(\times 2), 0.056,0.060,0.061,0.062,0.064,90.787, \\
217.233,516.877,2365.565,2562.612,3045.280,3087.675,3723.430,4057.062, \\
4175.139,4796.665,4955.207,5137.127,5521.505,5919.948,6130.132,6362.714, \\
6468.376,6774.743,7039.390,7092.200,8069.751,8398.881,8888.330,9432.107, \\
9461.600,11838.385,11867.153,12618.624,12645.769,13342.793,13404.201, \\
14738.302,14772.460,19224.064,19224.884,20274.302,20275.170,21473.291, \\
21475.615,22952.790,22954.359,24352.279,24354.107,31375.590,31376.230, \\
33279.019,33279.082,34140.544,34140.630,47536.547,47536.552,48953.611, \\
48953.621,67824.098(\times 2), 83151.688(\times 2)\end{array}$ \\
\hline $\mathcal{M}_{v} / g$ & $\begin{array}{l}-218.208(\times 2),-187.698(\times 2),-157.047,-157.037,-144.743,-144.730, \\
-121.434,-121.365,-115.966,-115.876,-93.728,-93.056,-90.474,-88.115, \\
-83.478,-83.014,-81.304,-71.795,-66.857,-64.519,-57.676,-56.563, \\
-50.546,-35.243,-19.608,-9.015,0.000(\times 72), 12.554,19.803,31.607,47.845, \\
53.984,57.193,62.572,67.152,70.946,78.488,80.675,81.570,86.750,88.060, \\
92.796,93.341,112.027,112.197,124.756,124.764,148.409,148.415,154.222, \\
154.235,189.074(\times 2), 226.244(\times 2)\end{array}$ \\
\hline$A_{1}$ & $\begin{array}{l}-131.088,-131.067,-100.148,-99.936,-73.728,-72.308,-49.538,-41.329, \\
40.453,46.306,66.617,68.915,100.098,100.376,138.177,138.200\end{array}$ \\
\hline$A_{3}$ & $\begin{array}{l}-393.263,-393.202,-300.443,-299.807,-276.410(\times 2),-233.511(\times 2), \\
-221.185,-216.925,-207.043(\times 2),-201.798,-201.797,-174.942,-174.941, \\
-171.416,-171.415,-167.016,-167.015,-148.615,-145.747,-145.741, \\
-140.362,-140.354,-133.141,-133.130,-129.546,-129.535,-123.986, \\
-111.816,-111.291,-109.753,-109.270,-105.010,-104.943,-95.598, \\
-95.306,-91.231,-90.931,-88.676,-86.493,-85.429,-83.220,-82.066, \\
-78.695,-73.403,-69.292,-67.084,-66.508,-60.963,-60.853,-57.830, \\
-47.289,-45.267,-41.192,-34.857,-26.875,-23.847,-21.499,-14.840, \\
-5.302,-4.751,-0.116,1.640,6.372,10.651,14.461,17.006,27.662,32.377, \\
34.649,39.470,46.301,47.768,52.006,56.956,58.208,61.574,66.122,67.208, \\
70.832,77.183,78.591,84.066,84.852,87.246,88.210,91.419,92.474,98.311, \\
98.979,101.874,102.240,104.968,105.362,112.949,113.114,121.360,131.287, \\
131.308,135.409,135.423,138.918,139.897,139.946,142.421,142.429, \\
163.461,163.462,171.404(\times 2), 174.446,174.447,199.850,202.377(\times 2), 206.745, \\
212.121(\times 2), 238.612(\times 2), 278.626(\times 2), 300.293,301.127,414.531,414.600\end{array}$ \\
\hline
\end{tabular}

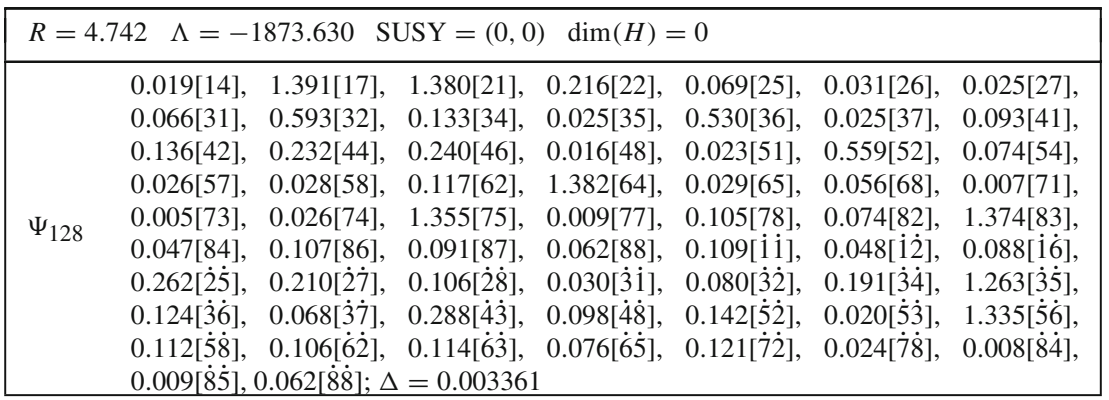




\begin{tabular}{|c|c|}
\hline $\mathcal{M}_{s} / g^{2}$ & $\begin{array}{l}-2485.023,-1850.535,-1618.214,-1606.209,-1087.060,-1087.055,-732.081, \\
-732.079,-650.519,-304.625,-304.623,-142.377,-120.675,-98.965,-0.024, \\
-0.022,-0.019(\times 3),-0.017,-0.015,-0.014(\times 2),-0.013,-0.012,-0.011(\times 2), \\
-0.010(\times 2),-0.008(\times 2),-0.007,-0.006(\times 2),-0.005,-0.004,-0.003(\times 2), \\
-0.002(\times 3), 0.000(\times 3), 0.001,0.002(\times 2), 0.003(\times 2), 0.004,0.005(\times 2), 0.007, \\
0.008(\times 2), 0.009,0.010,0.011(\times 2), 0.012,0.013(\times 2), 0.015,0.016,0.017(\times 2), \\
0.020,0.022,0.025,0.026,503.468,752.704,1111.739,1111.742,1166.711, \\
1696.130,3499.829,3499.832,3510.849,3747.715,4343.707,4734.816,4734.821, \\
4978.036,5374.885,5386.191,5386.193,5645.056,5795.818,5795.822,6454.202, \\
6877.151,6877.152,7532.979,8151.959,8660.982,8660.986,8762.872,8810.335, \\
8973.065,9118.387,9197.316,9386.148,9386.153,9798.141,10094.414,10306.616, \\
10379.428,10379.436,10516.572,10819.830,10819.840,11241.811,11348.975, \\
11732.386,11732.394,11921.702,12777.064,13240.475,13268.701,14530.494, \\
14530.499,15424.968,15424.970,18576.797,18654.629,19845.104,19860.630\end{array}$ \\
\hline $\mathcal{M}_{v} / g$ & $\begin{array}{l}-117.811,-117.077,-107.720,-106.097,-106.031,-102.968,-102.762, \\
-98.932,-98.440,-98.321,-95.986,-92.161,-92.097,-90.488,-89.064, \\
-85.559,-84.633,-79.903,-79.377,-73.100,-71.413,-64.382,-57.438, \\
-53.159,-45.986,-41.914,-40.738,-19.887,0.000(\times 72), 19.887,40.738, \\
41.914,45.986,53.159,57.438,64.382,71.413,73.100,79.377,79.903,84.633, \\
85.559,89.064,90.488,92.097,92.161,95.986,98.321,98.440,98.932,102.762, \\
102.968,106.031,106.097,107.720,117.077,117.811\end{array}$ \\
\hline$A_{1}$ & $\begin{array}{l}-81.429,-79.330,-77.106,-74.317,-72.760,-68.109,-57.560,-45.089, \\
45.089,57.560,68.109,72.760,74.317,77.106,79.330,81.429\end{array}$ \\
\hline$A_{3}$ & $\begin{array}{l}-244.288,-237.991,-231.317,-222.952,-218.280,-204.326,-172.680, \\
-141.405,-140.839,-135.267,-131.017,-129.897,-127.775,-127.669, \\
-118.982,-117.915,-117.720,-115.913,-114.229,-113.578,-113.434, \\
-110.522,-108.982,-108.673,-106.813,-106.141,-104.999,-104.549, \\
-102.913,-102.088,-102.074,-99.894,-97.595,-96.148,-94.768,-94.282, \\
-93.748,-93.719,-91.408,-90.992,-86.694,-86.362,-85.605,-82.823, \\
-78.925,-77.746,-76.573,-67.709,-63.642,-60.355,-60.237,-56.227, \\
-55.510,-53.256,-48.011,-46.231,-40.525,-31.627,-29.640,-28.605, \\
-20.754,-16.659,-12.174,-6.149,6.149,12.174,16.659,20.754,28.605,29.640, \\
31.627,40.525,46.232,48.011,53.256,55.509,56.227,60.237,60.355,63.642, \\
67.709,76.573,77.746,78.925,82.823,85.605,86.362,86.694,90.992,91.408, \\
93.719,93.748,94.282,94.768,96.148,97.595,99.894,102.074,102.088,102.913, \\
104.549,104.999,106.141,106.813,108.673,108.982,110.522,113.434,113.578, \\
114.229,115.913,117.720,117.915,118.982,127.669,127.775,129.897,131.017, \\
135.267,140.839,141.405,172.680,204.326,218.280,222.952,231.317,237.991, \\
244.288\end{array}$ \\
\hline
\end{tabular}

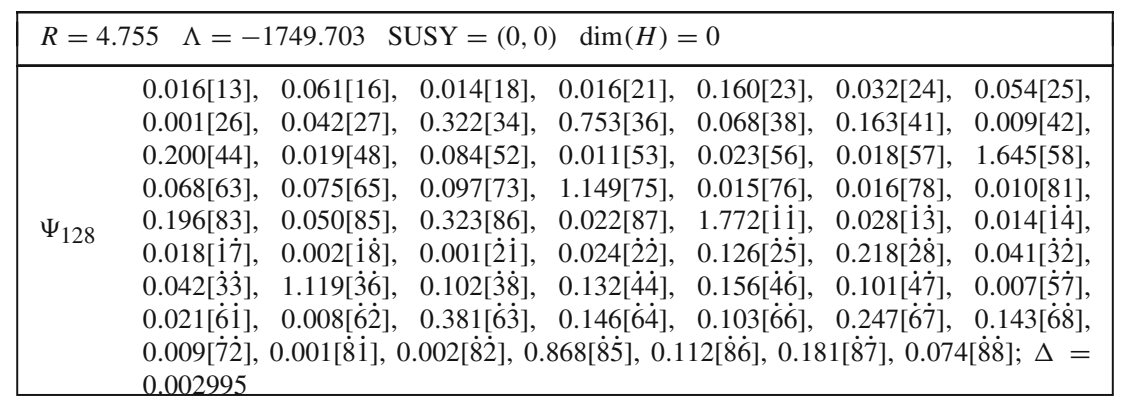




\begin{tabular}{|c|c|}
\hline $\mathcal{M}_{s} / g^{2}$ & $\begin{array}{l}-2517.739,-2058.950,-1779.459,-1599.551,-1379.488,-1130.732,-952.426, \\
-806.439,-647.256,-483.033,-305.959,-244.079,-114.696,-41.651,-0.057, \\
-0.054,-0.052,-0.049,-0.047,-0.046,-0.044,-0.043,-0.040,-0.038, \\
-0.034,-0.029,-0.020(\times 2),-0.017,-0.016,-0.014,-0.011,-0.010(\times 2), \\
-0.008,-0.006(\times 2),-0.005,-0.003(\times 2),-0.002,0.000(\times 3), 0.001,0.002,0.004, \\
0.005(\times 2), 0.007,0.008,0.010(\times 2), 0.013(\times 2), 0.016,0.018,0.019,0.029,0.034, \\
0.037,0.039,0.041,0.045,0.047,0.048,0.049,0.052,0.053,0.057,380.070,512.245, \\
1586.558,2087.582,2657.308,2865.183,3230.119,3343.879,4296.199,4368.137, \\
5024.953,5442.757,5769.490,6153.216,6483.249,6488.290,6753.644,6988.232, \\
7122.623,7594.750,8190.292,8331.617,8343.804,8828.227,8950.104,9103.968, \\
10594.218,10680.740,11528.788,11765.796,14259.174,14266.422,15206.963, \\
15234.130,17065.891,17072.717,17335.047,17339.845,19850.202,19854.324, \\
22095.500,22103.189,25798.020,25799.846,28370.472,28372.256,31154.351, \\
31154.796,32494.065,32494.244,45584.278,45584.378,46973.098,46973.108, \\
67251.457,67251.462,74545.234,74545.235\end{array}$ \\
\hline $\mathcal{M}_{v} / g$ & $\begin{array}{l}-217.803(\times 2),-182.198,-182.197,-150.959,-150.935,-139.566,-139.555, \\
-130.042,-130.004,-107.023,-106.833,-98.135,-97.902,-89.946,-89.353, \\
-82.867,-79.315,-75.941,-72.861,-69.247,-65.761,-62.155,-56.576, \\
-48.712,-32.349,-19.177,-14.287,0.000(\times 72), 14.287,19.177,32.349,48.712, \\
56.576,62.155,65.761,69.247,72.861,75.941,79.315,82.867,89.353,89.946, \\
97.902,98.135,106.833,107.023,130.004,130.042,139.555,139.566,150.935, \\
150.959,182.197,182.198,217.803(\times 2)\end{array}$ \\
\hline$A_{1}$ & $\begin{array}{l}-133.404,-133.386,-98.882,-98.545,-70.048,-66.752,-47.774,-40.346, \\
40.346,47.774,66.752,70.048,98.545,98.882,133.386,133.404\end{array}$ \\
\hline$A_{3}$ & $\begin{array}{l}-400.213,-400.159,-296.647,-295.636,-269.455(\times 2),-224.224(\times 2), \\
-212.681(\times 2),-210.144,-200.255,-189.604(\times 2),-176.042,-176.040, \\
-169.649,-169.645,-157.910,-157.900,-144.566,-144.557,-143.321, \\
-138.606,-138.547,-132.797,-132.777,-129.927,-129.888,-121.038, \\
-111.797,-111.185,-107.143,-106.205,-101.628,-101.375,-95.446, \\
-94.130,-92.720,-91.637,-90.041,-88.350,-87.334,-85.140,-84.975, \\
-82.030,-74.870,-72.140,-65.902,-62.195,-58.819,-57.111,-52.331, \\
-50.413,-47.749,-43.642,-37.595,-29.725,-23.295,-19.818,-15.021, \\
-11.556,-5.601,-3.097,3.097,5.601,11.556,15.021,19.818,23.295,29.725, \\
37.595,43.642,47.749,50.413,52.331,57.111,58.819,62.195,65.902,72.140, \\
74.870,82.030,84.975,85.140,87.334,88.350,90.041,91.637,92.720,94.130, \\
95.446,101.375,101.628,106.205,107.143,111.185,111.797,121.038,129.889, \\
129.927,132.777,132.797,138.547,138.606,143.321,144.557,144.566,157.900, \\
157.910,169.645,169.649,176.040,176.042,189.604(\times 2), 200.255,210.144, \\
212.681(\times 2), 224.224(\times 2), 269.455(\times 2), 295.636,296.647,400.159,400.213\end{array}$ \\
\hline
\end{tabular}

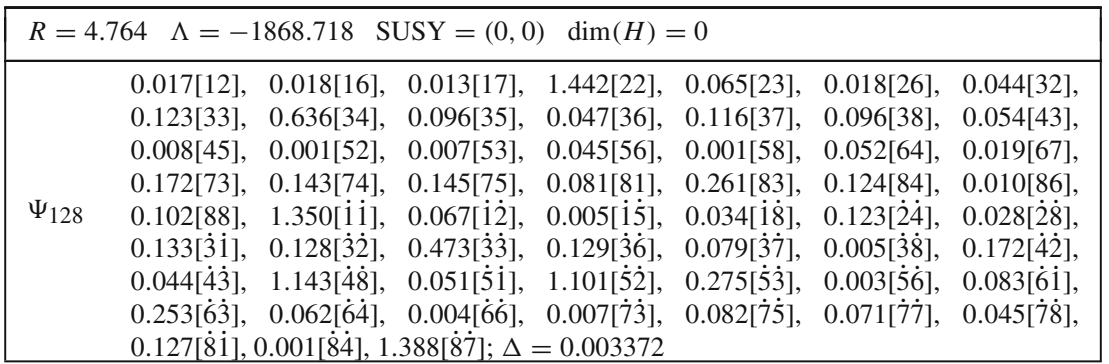




\begin{tabular}{|c|c|}
\hline $\mathcal{M}_{s} / g^{2}$ & $\begin{array}{l}-2649.076,-2496.336,-2012.031,-1833.505,-1559.208,-1037.631, \\
-988.450,-654.617,-451.322,-331.241,-109.011,-81.206,-50.753,-0.027, \\
-0.026(\times 2),-0.025,-0.023,-0.020,-0.019,-0.017(\times 2),-0.016,-0.014(\times 2), \\
-0.013,-0.012(\times 2),-0.011,-0.010,-0.009,-0.008,-0.007,-0.006(\times 2), \\
-0.005,-0.004(\times 2),-0.003(\times 2),-0.002,-0.001,0.000(\times 3), 0.002(\times 2), \\
0.003,0.004(\times 2), 0.006(\times 2), 0.007(\times 2), 0.008,0.009,0.010,0.011,0.012(\times 2), \\
0.013,0.015,0.016,0.017,0.018,0.019(\times 2), 0.021,0.024,154.268,686.541, \\
888.599,976.413,1176.633,1971.693,2105.848,2615.353,2781.419,3837.815, \\
3878.870,4148.718,4696.523,4713.501,5289.190,5310.958,5691.435,6024.681, \\
6440.975,6513.431,6937.773,7195.390,7513.306,7725.437,8301.575,8363.915, \\
8776.857,8817.161,9090.990,9321.487,9610.605,9705.548,9944.162,10109.587, \\
10370.778,10658.777,11069.943,11865.320,12037.583,12092.852,12420.459, \\
12598.912,12827.720,13175.507,13234.787,14528.060,15104.500,15531.496, \\
15761.299,16401.342,17489.079,18461.395,19572.664,20176.123,20736.623, \\
21395.079,21522.542,23869.799,23941.546\end{array}$ \\
\hline $\mathcal{M}_{v} / g$ & $\begin{array}{l}-126.986,-126.561,-124.175,-122.884,-122.239,-119.659,-113.814, \\
-113.258,-102.313,-100.661,-93.166,-92.057,-89.790,-87.700,-84.395, \\
-84.027,-83.535,-81.840,-80.286,-77.122,-70.205,-63.099,-62.685, \\
-58.774,-50.995,-28.576,-21.748,-16.429,0.000(\times 72), 16.429,21.748, \\
28.576,50.995,58.774,62.686,63.099,70.205,77.122,80.286,81.840,83.535, \\
84.027,84.395,87.700,89.790,92.057,93.166,100.661,102.313,113.258,113.814, \\
119.659,122.239,122.884,124.175,126.560,126.986\end{array}$ \\
\hline$A_{1}$ & $\begin{array}{l}-96.810,-92.823,-83.892,-79.171,-61.411,-60.238,-57.232,-40.697, \\
40.697,57.232,60.238,61.411,79.171,83.892,92.823,96.810\end{array}$ \\
\hline$A_{3}$ & $\begin{array}{l}-290.431,-278.468,-251.676,-237.512,-184.232,-180.714,-171.697, \\
-153.961,-153.672,-147.970,-147.287,-137.185,-136.663,-133.620, \\
-132.427,-129.004,-128.319,-126.036,-125.137,-122.092,-120.071, \\
-118.710,-118.030,-116.275,-115.165,-114.154,-112.326,-110.339, \\
-108.986,-105.714,-104.074,-103.848,-101.629,-100.700,-98.351, \\
-96.991,-94.179,-93.821,-92.302,-86.570,-85.836,-85.183,-83.921, \\
-83.048,-81.581,-78.931,-75.040,-72.565,-69.679,-67.604,-64.262, \\
-54.376,-53.189,-51.011,-44.029,-38.675,-34.438,-33.468,-29.648, \\
-22.525,-10.850,-7.635,-5.526,-5.073,5.073,5.526,7.635,10.850,22.525, \\
29.648,33.468,34.438,38.674,44.029,51.011,53.189,54.376,64.262,67.604, \\
69.679,72.565,75.040,78.931,81.581,83.048,83.921,85.183,85.836,86.570, \\
92.302,93.821,94.179,96.991,98.351,100.700,101.629,103.848,104.074,105.714, \\
108.986,110.339,112.326,114.154,115.165,116.275,118.030,118.710,120.071, \\
122.092,125.137,126.036,128.319,129.004,132.427,133.620,136.663,137.185, \\
147.287,147.970,153.672,153.961,171.697,180.714,184.232,237.512,251.676, \\
278.468,290.431\end{array}$ \\
\hline
\end{tabular}

\begin{tabular}{|c|c|c|c|c|c|c|}
\hline \multicolumn{7}{|c|}{$R=4.781 \quad \Lambda=-1880.634 \quad \operatorname{SUSY}=(0,0) \quad \operatorname{dim}(H)=0$} \\
\hline$\Psi_{128}$ & $\begin{array}{l}0.004[11], \\
0.070[33], \\
0.124[44], \\
0.076[62], \\
0.428[77], \\
0.262[\dot{2} \dot{4}], \\
0.553[\dot{5} \dot{4}], \\
0.100[\dot{7} \dot{4}],\end{array}$ & $\begin{array}{cc}1.501[13], & 0.079[15], \\
0.035[34], & 1.486[35], \\
0.099[45], & 0.019[48], \\
1.480[64], & 0.212[68], \\
1.331[82], & 0.092[84], \\
0.770[\dot{3} \dot{1}], & 0.091[\dot{3} \dot{8}], \\
0.045[\dot{5} 5], & 0.032[\dot{5} 7], \\
0.064[\dot{7} \dot{8}], & 0.332[\dot{8} \dot{2}], 0 .\end{array}$ & $\begin{array}{l}0.359[21], \\
0.007[36], \\
0.109[51], \\
0.752[71], \\
0.096[\dot{1} \dot{2}], \\
0.337[\dot{4} \dot{4}], \\
0.032[\dot{5} \dot{8}], \\
94[\dot{8} \dot{3}], 0.00\end{array}$ & $\begin{array}{l}0.020[23], \\
0.036[38], \\
0.019[52], \\
0.075[72], \\
1.224[\dot{1} \dot{3}], \\
0.056[\dot{4} \dot{6}], \\
0.023[\dot{6} \dot{7}], \\
9[\dot{8} \dot{5}] ; \Delta=(\end{array}$ & $\begin{array}{l}0.239[27], \\
0.168[41], \\
0.031[55], \\
0.169[75], \\
0.068[\dot{1} \dot{6}], \\
0.017[\dot{4} \dot{7}], \\
0.120[\dot{6} \dot{8}], \\
0.003350\end{array}$ & $\begin{array}{l}0.438[28], \\
0.025[43], \\
0.290[57], \\
0.183[76], \\
0.030[\dot{1} \overline{7}], \\
1.558[\dot{4} \dot{8}], \\
0.132[\dot{7} \dot{3}],\end{array}$ \\
\hline
\end{tabular}




\begin{tabular}{|c|c|}
\hline $\mathcal{M}_{s} / g^{2}$ & $\begin{array}{l}-2616.303,-2069.943,-1718.324,-1460.454,-1250.731,-1104.000,-957.240, \\
-921.127,-750.903,-632.636,-205.856,-89.074,-19.082,-0.046,-0.039, \\
-0.033,-0.030,-0.028,-0.026(\times 2),-0.025,-0.023,-0.022,-0.020,-0.018, \\
-0.017,-0.015,-0.014,-0.013,-0.012,-0.011,-0.010,-0.009,-0.008, \\
-0.007,-0.004(\times 2),-0.003,-0.002,0.000(\times 2), 0.001,0.002,0.003,0.004,0.006, \\
0.007(\times 2), 0.009(\times 2), 0.011(\times 2), 0.014,0.015,0.017,0.019(\times 2), 0.020,0.022, \\
0.023(\times 2), 0.025,0.026,0.028,0.031,0.033,0.034,0.041,0.044,27.445,309.122, \\
407.455,1075.411,1862.898,2080.331,2703.664,3781.492,3975.100,4623.428, \\
4749.663,4807.918,5505.133,5695.958,5832.278,6085.094,6534.530,7069.836, \\
7165.248,7278.160,7791.662,8194.337,8249.439,8387.469,8612.533,8967.499, \\
9105.391,9417.385,9568.666,10897.854,11410.634,11660.977,11718.058, \\
13108.783,13210.703,13740.346,13841.687,15329.038,15334.179,16285.811, \\
16334.703,17643.257,17671.610,21439.060,21450.922,22799.505,22810.550, \\
24078.461,24080.049,26912.108,26913.959,33778.923,33779.876,39176.191, \\
39176.515,49424.694,49424.740,53789.467,53789.499\end{array}$ \\
\hline $\mathcal{M}_{v} / g$ & $\begin{array}{l}-195.734(\times 2),-154.711,-154.707,-134.989,-134.926,-126.188,-126.075, \\
-124.314,-124.225,-105.915,-104.880,-98.784,-97.011,-91.929,-86.321, \\
-85.815,-83.099,-77.848,-74.270,-70.606,-63.903,-60.843,-53.377, \\
-48.915,-46.030,-22.536,-16.063,0.000(\times 72), 14.019,18.021,48.003,57.034, \\
63.216,64.678,68.038,70.943,74.446,78.959,83.398,85.786,92.481,93.082, \\
96.035,97.254,102.810,103.446,110.836,111.066,126.694,126.838,135.832, \\
135.898,158.172,158.178,192.292,192.293\end{array}$ \\
\hline$A_{1}$ & $\begin{array}{l}-124.265,-124.129,-91.149,-90.903,-64.933,-58.209,-52.022,-44.952, \\
41.914,50.251,62.685,71.878,91.971,93.120,119.299,119.444\end{array}$ \\
\hline$A_{3}$ & $\begin{array}{l}-372.796,-372.388,-273.447,-272.708,-228.141(\times 2),-205.002,-205.001, \\
-194.799,-194.719,-194.717,-174.626,-162.185,-162.179,-159.762, \\
-159.754,-156.067,-153.812,-153.801,-138.404,-138.308,-134.857, \\
-134.281,-134.106,-128.482,-128.335,-120.707,-120.563,-119.451, \\
-119.083,-110.808,-108.851,-107.710,-106.940,-101.821,-100.016, \\
-97.496,-96.876,-94.985,-91.214,-89.466,-89.042,-87.436,-86.560, \\
-85.191,-82.546,-78.059,-75.505,-71.380,-69.715,-63.459,-61.427, \\
-52.714,-51.719,-49.262,-45.567,-42.722,-28.144,-20.326,-19.725, \\
-11.655,-10.574,-7.271,-5.983,-1.457,5.305,10.423,16.615,22.136,25.093, \\
32.392,40.053,45.099,47.269,49.549,54.921,57.259,64.255,68.177,69.894, \\
75.233,77.910,82.445,85.487,86.490,88.994,90.202,92.514,94.006,95.611, \\
97.180,101.822,102.362,103.791,104.768,106.219,112.396,112.886,118.023, \\
118.597,121.609,121.981,125.742,130.308,130.345,133.247,133.521,140.676, \\
140.831,150.319,150.326,150.752,161.467,161.482,164.636,164.645,185.738, \\
185.740,188.056,196.755(\times 2), 215.635,233.399(\times 2), 275.914,279.360,357.896, \\
358.331\end{array}$ \\
\hline
\end{tabular}

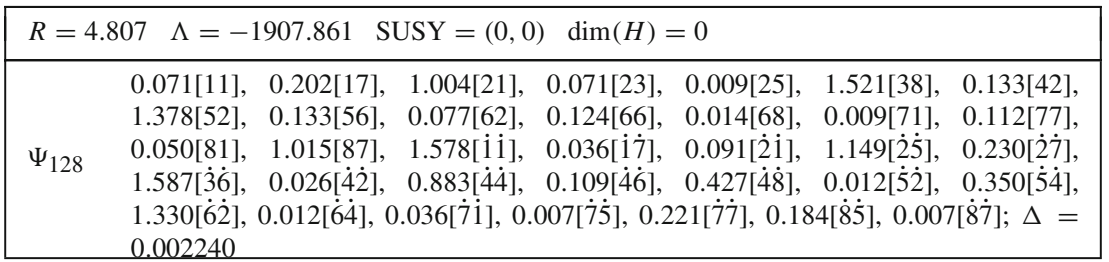




\begin{tabular}{|c|c|}
\hline $\mathcal{M}_{s} / g^{2}$ & $\begin{array}{l}-2835.573,-2425.194,-2359.887,-1629.910,-1549.716,-1385.040, \\
-1291.164,-1198.826,-669.686,-513.631,-95.002,-53.574,-38.344, \\
-0.036,-0.034,-0.028,-0.027,-0.024,-0.023(\times 2),-0.020,-0.019,-0.017, \\
-0.016,-0.014,-0.013,-0.012,-0.011,-0.010,-0.009,-0.008,-0.007, \\
-0.006,-0.005,-0.004,-0.003,0.000(\times 3), 0.001,0.002,0.003(\times 2), 0.005(\times 2), \\
0.006,0.007(\times 2), 0.009(\times 2), 0.010,0.012(\times 2), 0.013,0.014,0.015,0.016,0.017, \\
0.018,0.019,0.020,0.021,0.025,0.026,0.027,0.030,0.033,0.036,0.040,34.486, \\
49.291,834.015,900.058,2260.764,2486.311,2683.811,2803.477,3174.754, \\
4287.631,4596.840,4786.099,5352.860,6315.838,6837.324,7298.927,7445.141, \\
7573.461,7696.169,7918.741,7979.183,9001.354,9036.533,9229.719,9631.820, \\
9966.669,10051.669,10425.772,10790.868,11527.518,11529.068,15449.393, \\
15450.390,15800.808,15810.380,17847.217,17867.865,18603.935,18645.126, \\
19434.760,19500.065,19921.043,19979.546,24617.274,24733.953,25610.751, \\
25664.733,27326.343,27331.363,28712.235,28732.148,30228.624,30230.781, \\
33591.803,33597.513,42524.493,42525.410,44786.304,44787.571\end{array}$ \\
\hline $\mathcal{M}_{v} / g$ & $\begin{array}{l}-179.153,-179.148,-175.193,-175.186,-140.774,-140.624,-137.056, \\
-136.983,-115.448,-114.731,-100.817,-100.644,-98.136,-97.441,-96.016, \\
-89.122,-88.984,-86.374,-83.039,-80.698,-70.203,-68.149,-53.207, \\
-48.392,-47.274,-31.464,-22.407,-16.968,0.000(\times 72), 16.968,22.407, \\
31.464,47.274,48.392,53.207,68.149,70.203,80.698,83.039,86.374,88.984, \\
89.122,96.016,97.441,98.136,100.644,100.817,114.731,115.448,136.983, \\
137.056,140.624,140.774,175.186,175.193,179.148,179.153\end{array}$ \\
\hline$A_{1}$ & $\begin{array}{l}-136.382,-136.365,-76.003,-72.628,-68.684,-56.884,-47.711,-46.430, \\
46.430,47.711,56.884,68.684,72.628,76.004,136.365,136.382\end{array}$ \\
\hline$A_{3}$ & $\begin{array}{l}-409.147,-409.095,-228.010,-217.884,-211.671(\times 2),-206.052,-186.444, \\
-186.430,-185.456,-185.440,-172.811,-172.799,-172.743,-172.718, \\
-170.652,-157.373,-157.107,-154.348,-154.121,-143.133,-140.903, \\
-140.740,-139.291,-133.340,-133.187,-130.650,-130.579,-123.264, \\
-119.873,-117.721,-113.655,-111.774,-111.495,-109.272,-106.209, \\
-101.087,-99.276,-98.885,-98.872,-94.784,-92.758,-91.352,-89.152, \\
-83.738,-83.122,-79.809,-78.278,-71.557,-66.996,-62.158,-60.880, \\
-55.518,-49.448,-43.247,-36.204,-31.285,-31.033,-23.199,-22.864, \\
-16.437,-15.221,-13.009,-5.961,5.961,13.009,15.221,16.437,22.864,23.199, \\
31.033,31.284,36.204,43.247,49.448,55.518,60.880,62.158,66.996,71.557, \\
78.278,79.809,83.122,83.738,89.152,91.352,92.758,94.784,98.872,98.885, \\
99.276,101.087,106.209,109.272,111.495,111.774,113.655,117.721,119.873, \\
123.264,130.579,130.650,133.187,133.340,139.291,140.740,140.903,143.133, \\
154.121,154.348,157.107,157.373,170.652,172.718,172.743,172.799,172.811, \\
185.440,185.456,186.430,186.444,206.052,211.671(\times 2), 217.884,228.011, \\
409.095,409.147\end{array}$ \\
\hline
\end{tabular}

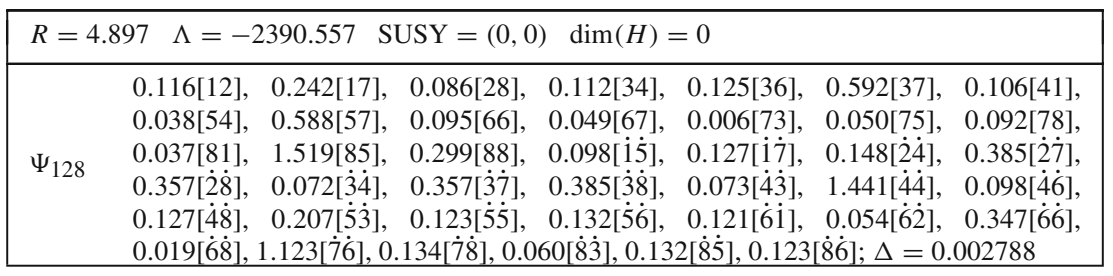




\begin{tabular}{|c|c|}
\hline $\mathcal{M}_{s} / g^{2}$ & $\begin{array}{l}-4046.781,-3075.315,-1894.380,-1783.548,-1724.353,-1091.587,-741.322, \\
-555.864,-155.581,-22.853,-0.029,-0.028,-0.024,-0.023,-0.022,-0.020, \\
-0.019,-0.016,-0.014,-0.013,-0.012,-0.011,-0.010,-0.009(\times 2),-0.008, \\
-0.007(\times 2),-0.006,-0.005,-0.004,-0.002,-0.001(\times 3), 0.000,0.001, \\
0.002(\times 2), 0.003,0.004,0.005,0.006,0.007(\times 2), 0.008,0.009,0.010,0.011, \\
0.012,0.013,0.014(\times 2), 0.016(\times 2), 0.017,0.018,0.022(\times 2), 0.023(\times 2), 0.025, \\
0.028,0.032,0.034,0.035,139.455,151.768,306.307,307.123,1709.638,2445.857, \\
2509.746,2779.427,3118.893,3585.043,3635.997,4648.968,4680.715,5580.399, \\
5774.674,6212.180,7191.888,7255.703,8131.868,8198.549,8578.785,8817.259, \\
9218.601,9286.780,9590.709,9978.535,10015.814,10317.657,10813.680, \\
11115.751,11283.913,11515.559,12266.697,12292.783,12784.851,12807.229, \\
13558.652,13611.181,14249.766,14334.273,14857.120,15288.674,15658.137, \\
15668.828,15765.993,16357.222,16720.523,16987.163,16996.133,17864.294, \\
18504.434,18733.101,19902.835,20212.828,20457.711,21180.343,23801.954, \\
24190.307,26360.768,26635.958,30662.051,30764.924\end{array}$ \\
\hline $\mathcal{M}_{v} / g$ & $\begin{array}{l}-144.198,-143.793,-133.049,-132.516,-127.168,-124.965,-122.740, \\
-118.117,-115.906,-114.328,-110.550,-108.946,-108.227,-104.501, \\
-102.650,-98.573,-95.004,-92.561,-90.946,-89.863,-89.792,-80.996, \\
-72.900,-71.285,-64.047,-34.056,-30.835,-15.503,0.000(\times 72), 15.503, \\
30.835,34.056,64.047,71.285,72.900,80.996,89.792,89.863,90.946,92.561, \\
95.004,98.573,102.650,104.501,108.227,108.946,110.550,114.328,115.906, \\
118.117,122.740,124.965,127.168,132.516,133.049,143.793,144.198\end{array}$ \\
\hline$A_{1}$ & $\begin{array}{l}-101.605,-99.648,-90.837,-88.846,-86.786,-68.037,-66.116,-53.280, \\
53.280,66.116,68.037,86.786,88.846,90.837,99.648,101.605\end{array}$ \\
\hline$A_{3}$ & $\begin{array}{l}-304.816,-298.944,-272.512,-266.539,-260.357,-204.112,-198.349, \\
-173.217,-172.923,-159.839,-159.786,-159.220,-149.430,-147.927, \\
-144.770,-143.926,-141.781,-141.227,-138.848,-138.792,-134.736, \\
-134.593,-131.902,-130.752,-128.189,-127.658,-126.153,-125.962, \\
-123.335,-120.485,-120.438,-117.732,-117.002,-114.300,-113.911, \\
-112.299,-109.416,-109.204,-108.875,-104.990,-103.562,-100.464, \\
-100.057,-95.215,-95.016,-92.496,-82.890,-80.250,-77.555,-76.115, \\
-74.208,-72.316,-65.510,-58.550,-54.533,-53.588,-51.117,-46.624, \\
-44.153,-33.512,-29.493,-20.396,-2.510,-2.349,2.349,2.510,20.396,29.493, \\
33.512,44.153,46.624,51.117,53.588,54.533,58.550,65.510,72.316,74.208, \\
76.115,77.555,80.250,82.890,92.496,95.016,95.215,100.057,100.464,103.562, \\
104.990,108.875,109.204,109.416,112.299,113.911,114.300,117.002,117.732, \\
120.438,120.485,123.335,125.962,126.153,127.658,128.189,130.752,131.902, \\
134.593,134.736,138.792,138.848,141.227,141.781,143.926,144.770,147.927, \\
149.430,159.220,159.786,159.839,172.923,173.217,198.348,204.112,260.357, \\
266.539,272.512,298.944,304.816\end{array}$ \\
\hline
\end{tabular}

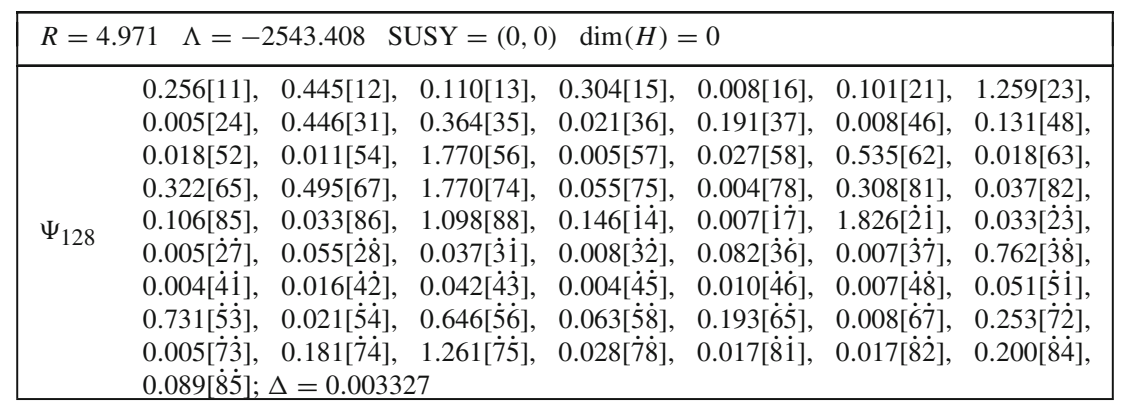




\begin{tabular}{|c|c|}
\hline $\mathcal{M}_{s} / g^{2}$ & $\begin{array}{l}-3394.441,-3226.501,-2727.892,-1914.828,-1597.160,-1255.559,-957.167, \\
-765.420,-435.441,-145.799,-82.234,-0.061,-0.054,-0.053,-0.045, \\
-0.044,-0.042,-0.036,-0.035,-0.033,-0.029,-0.025,-0.024,-0.023, \\
-0.021,-0.020,-0.017,-0.016,-0.014,-0.011,-0.010,-0.009,-0.008, \\
-0.006(\times 2),-0.004,-0.002,0.000,0.002(\times 2), 0.004(\times 2), 0.005(\times 2), 0.007, \\
0.010,0.011,0.013,0.015,0.018,0.019,0.020,0.021,0.024,0.025,0.026,0.027, \\
0.030,0.035,0.038,0.040,0.042,0.044,0.046,0.050,0.059,0.062,36.163, \\
1310.326,1837.094,2703.075,3606.152,4173.078,4237.464,5111.187,5437.357, \\
5749.376,6171.912,6781.767,8073.141,8529.610,9864.960,10739.552,10765.915, \\
11118.472,11492.148,11915.864,12142.661,12259.701,12491.404,12556.317, \\
13023.716,13031.738,13890.067,14321.764,14985.142,15209.229,15755.352, \\
15966.748,16389.351,20550.508,20554.854,21183.204,21195.272,22672.104, \\
22788.569,22832.993,22907.643,25625.790,25765.887,30943.694,30955.566, \\
32175.191,32177.666,33766.153,33770.458,39142.427,39145.404,40296.512, \\
40297.367,53241.154,53241.936,53449.606,53450.102,82790.824,82790.920,\end{array}$ \\
\hline $\mathcal{M}_{v} / g$ & $\begin{array}{l}-232.997,-232.995,-204.951,-204.949,-168.052,-167.984,-158.917, \\
-158.857,-150.687,-150.497,-127.389,-125.834,-124.215,-121.327, \\
-113.127,-108.872,-103.777,-101.844,-99.879,-93.289,-90.512,-87.225, \\
-80.586,-75.439,-74.087,-50.691,-20.078,-15.682,0.000(\times 72), 15.682, \\
20.078,50.691,74.087,75.439,80.586,87.226,90.512,93.289,99.879,101.844, \\
103.777,108.872,113.127,121.327,124.215,125.834,127.389,150.497,150.687, \\
158.857,158.917,167.984,168.052,204.949,204.952,232.995,232.997\end{array}$ \\
\hline$A_{1}$ & $\begin{array}{l}-148.165,-148.032,-108.003,-107.343,-91.212,-82.704,-69.934,-66.173, \\
66.173,69.935,82.704,91.212,107.342,108.003,148.032,148.165\end{array}$ \\
\hline$A_{3}$ & $\begin{array}{l}-444.495,-444.096,-324.008,-322.028,-291.126(\times 2),-273.637,-248.113, \\
-240.756,-240.755,-237.163,-237.160,-210.897,-210.893,-209.803, \\
-206.184,-206.177,-198.520,-182.192,-182.172,-176.935,-176.895, \\
-171.542,-171.491,-158.599,-158.458,-152.609,-152.542,-146.897, \\
-146.714,-134.873,-133.588,-132.077,-130.948,-129.145,-126.063, \\
-121.909,-119.626,-117.260,-116.225,-113.877,-112.638,-109.324, \\
-104.445,-102.907,-101.923,-100.307,-97.520,-97.137,-89.642,-82.586, \\
-76.613,-75.512,-73.074,-70.530,-65.912,-56.136,-48.038,-45.189, \\
-37.500,-31.519,-23.616,-19.054,-6.546,6.546,19.054,23.616,31.519, \\
37.500,45.188,48.038,56.136,65.912,70.530,73.074,75.512,76.613,82.586, \\
89.642,97.137,97.520,100.307,101.923,102.907,104.445,109.324,112.638, \\
113.877,116.225,117.260,119.626,121.909,126.063,129.145,130.948,132.077, \\
133.588,134.873,146.714,146.897,152.542,152.609,158.458,158.599,171.491, \\
171.542,176.895,176.935,182.172,182.192,198.520,206.177, \\
209.804,210.893,210.897,237.160,237.163,240.755,240.756,248.113,273.637,\end{array}$ \\
\hline
\end{tabular}

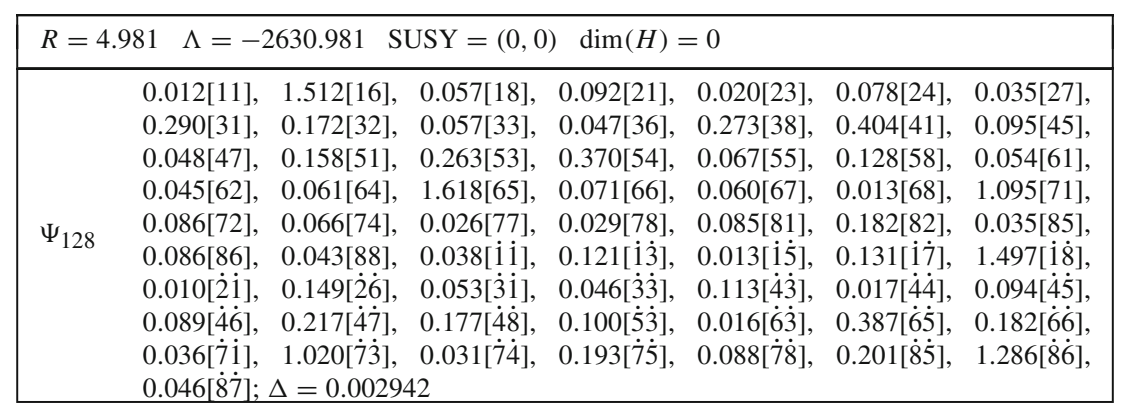




\begin{tabular}{|c|c|}
\hline $\mathcal{M}_{s} / g^{2}$ & $\begin{array}{l}-3708.821,-3384.663,-2948.594,-2357.028,-1293.442,-1196.842,-828.650, \\
-380.908,-94.665,-31.450,-0.062,-0.055,-0.052,-0.048,-0.045,-0.040, \\
-0.037(\times 2),-0.036,-0.033,-0.032,-0.028,-0.027,-0.025,-0.022,-0.020, \\
-0.019,-0.018,-0.017,-0.015,-0.014,-0.012,-0.011,-0.009,-0.008, \\
-0.006(\times 2),-0.004,-0.002,-0.001,0.000(\times 2), 0.002,0.004(\times 2), 0.008,0.009, \\
0.010,0.011,0.012,0.014,0.018,0.019(\times 2), 0.023,0.027,0.028,0.029,0.030, \\
0.034,0.037,0.041,0.044,0.047,0.050,0.057,172.097,439.781,1044.238, \\
1889.683,2899.505,2906.967,3808.311,3891.143,5127.525,5431.292,6013.111, \\
6207.031,6920.527,7976.039,8548.644,9117.637,9173.058,10545.488,10617.542, \\
11368.805,11390.321,11985.770,12169.184,12242.528,12472.225,12916.566, \\
13538.293,13837.195,14130.851,14487.392,14963.078,16416.776,16442.032, \\
17238.939,17838.474,18826.526,18930.580,19103.408,19620.605,19996.074, \\
20521.569,21849.530,22537.433,22643.900,24552.841,25396.651,27015.311, \\
27426.176,28663.409,28819.540,29327.088,29436.886,33255.972,33337.466, \\
35291.915,35404.994,39039.849,39113.854,51366.711,51371.750,58706.974, \\
58708.837\end{array}$ \\
\hline $\mathcal{M}_{v} / g$ & $\begin{array}{l}-187.730,-187.692,-171.486,-171.422,-157.514,-155.809,-148.440, \\
-145.169,-141.372,-137.464,-134.871,-130.719,-123.713,-123.009, \\
-112.412,-106.759,-105.222,-101.331,-99.340,-96.825,-94.560,-88.686, \\
-81.255,-77.593,-67.674,-51.933,-39.876,-15.277,0.000(\times 72), 15.277, \\
39.876,51.933,67.674,77.594,81.255,88.686,94.559,96.825,99.340,101.331, \\
105.222,106.759,112.412,123.008,123.713,130.719,134.871,137.464,141.372, \\
145.169,148.440,155.809,157.515,171.422,171.486,187.692,187.730\end{array}$ \\
\hline$A_{1}$ & $\begin{array}{l}-124.577,-123.508,-105.521,-98.216,-92.664,-83.364,-76.874,-74.421, \\
74.421,76.874,83.364,92.664,98.216,105.521,123.508,124.577\end{array}$ \\
\hline$A_{3}$ & $\begin{array}{l}-373.732,-370.525,-316.563,-294.647,-277.993,-250.091,-231.875, \\
-231.866,-230.623,-223.263,-216.803,-216.761,-194.383,-194.329, \\
-183.527,-183.292,-176.056,-175.834,-167.482,-166.226,-162.432, \\
-160.623,-157.132,-156.562,-154.265,-153.191,-147.351,-143.952, \\
-139.751,-139.267,-139.074,-135.527,-133.593,-133.441,-130.922, \\
-127.028,-126.385,-124.085,-120.852,-119.340,-118.799,-117.009, \\
-113.471,-109.191,-103.670,-100.479,-96.882,-93.855,-89.711,-84.088, \\
-83.160,-79.952,-74.852,-70.386,-64.680,-63.354,-59.375,-53.584, \\
-47.711,-38.697,-33.165,-24.796,-20.650,-4.335,4.335,20.650,24.796, \\
33.165,38.697,47.711,53.584,59.375,63.354,64.680,70.386,74.852,79.952, \\
83.160,84.088,89.711,93.855,96.882,100.479,103.670,109.191,113.471,117.009, \\
118.799,119.340,120.852,124.085,126.385,127.028,130.922,133.441,133.593, \\
135.527,139.074,139.267,139.751,143.952,147.351,153.191,154.266,156.562, \\
157.132,160.623,162.433,166.226,167.482,175.834,176.057,183.292,183.527, \\
194.329,194.383,216.761,216.803,223.263,230.623,231.866,231.875,250.091,\end{array}$ \\
\hline
\end{tabular}

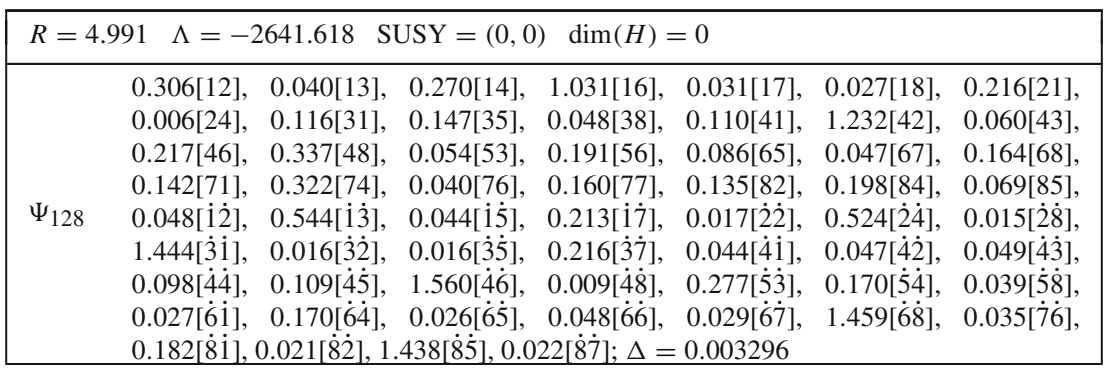




\begin{tabular}{|c|c|}
\hline $\mathcal{M}_{s} / g^{2}$ & $\begin{array}{l}-4145.867,-3240.417,-2830.698,-2465.663,-2188.398,-1549.679,-824.008, \\
-241.836,-202.185,-148.204,-61.052,-0.069,-0.061,-0.059,-0.057, \\
-0.055,-0.052,-0.050,-0.045,-0.043,-0.041,-0.035,-0.033,-0.027, \\
-0.025,-0.020,-0.016,-0.015,-0.014,-0.013,-0.012,-0.009(\times 2),-0.008, \\
-0.007,-0.004,-0.003,-0.002,0.000(\times 2), 0.002,0.003,0.005,0.007,0.008, \\
0.010,0.011,0.012,0.017(\times 2), 0.018,0.020,0.024,0.026,0.028,0.030,0.037,0.040, \\
0.045,0.049,0.050,0.051,0.054,0.056,0.060,0.064,0.072,1360.018,2041.869, \\
2205.571,2691.785,3732.340,4389.491,4515.843,5202.276,5362.769,6106.418, \\
6919.148,7051.317,7606.304,8242.431,9374.616,9923.021,9944.290,10051.662, \\
10285.299,12003.485,12229.765,12441.198,12902.432,13239.429,13867.080, \\
13867.520,14445.560,14791.368,15432.555,15732.813,16129.823,17238.450, \\
17691.254,17842.079,18622.392,19309.336,19616.124,21391.501,21410.633, \\
22764.978,22789.924,23456.683,23500.172,26242.760,26263.607,31489.220, \\
31527.670,34214.211,34217.094,35204.959,35215.218,37955.362,37977.793, \\
43061.024,43065.695,48095.419,48096.526,64426.334,64426.764,65568.370, \\
65568.675\end{array}$ \\
\hline $\mathcal{M}_{v} / g$ & $\begin{array}{l}-215.038,-215.034,-191.144,-191.131,-160.256,-159.938,-154.476, \\
-153.842,-139.228,-138.414,-130.889,-126.657,-119.932,-117.585, \\
-116.057,-112.635,-103.628,-101.241,-95.619,-93.282,-91.283,-87.738, \\
-83.625,-77.668,-72.810,-65.112,-39.564,-19.863,0.000(\times 72), 19.863, \\
39.564,65.112,72.810,77.668,83.625,87.738,91.283,93.282,95.619,101.241, \\
103.628,112.635,116.057,117.585,119.932,126.657,130.889,138.414,139.228, \\
153.843,154.476,159.938,160.256,191.131,191.144,215.034,215.038\end{array}$ \\
\hline$A_{1}$ & $\begin{array}{l}-146.898,-146.859,-98.188,-94.598,-87.094,-80.167,-73.457,-64.744, \\
64.744,73.457,80.167,87.094,94.597,98.188,146.859,146.898\end{array}$ \\
\hline$A_{3}$ & $\begin{array}{l}-440.694,-440.576,-294.564,-283.793,-261.282,-258.023,-258.022, \\
-240.500,-223.325,-223.323,-220.371,-218.189,-218.187,-198.541, \\
-198.510,-194.231,-191.886,-191.861,-184.250,-184.213,-168.807, \\
-168.527,-161.725,-160.525,-151.421,-149.422,-147.393,-145.992, \\
-145.039,-144.058,-140.598,-140.280,-136.151,-134.652,-129.123, \\
-127.177,-126.627,-126.455,-121.871,-121.265,-118.999,-116.471, \\
-115.129,-111.373,-106.382,-102.851,-98.419,-91.713,-90.070,-88.634, \\
-84.617,-82.078,-77.493,-71.102,-66.921,-58.593,-54.471,-52.895, \\
-44.891,-39.439,-31.354,-20.798,-18.488,-5.047,5.047,18.488,20.798, \\
31.354,39.439,44.891,52.895,54.471,58.593,66.921,71.102,77.493,82.078, \\
84.617,88.634,90.070,91.713,98.419,102.851,106.382,111.373,115.128,116.471, \\
118.999,121.265,121.871,126.455,126.627,127.177,129.123,134.652,136.151, \\
140.280,140.598,144.058,145.039,145.992,147.393,149.422,151.421,160.525, \\
161.725,168.527,168.807,184.213,184.250,191.861,191.886,194.231,198.510, \\
198.541,218.187,218.189,220.372,223.323,223.325,240.500,258.022,258.023, \\
261.282,283.792,294.564,440.576,440.695\end{array}$ \\
\hline
\end{tabular}

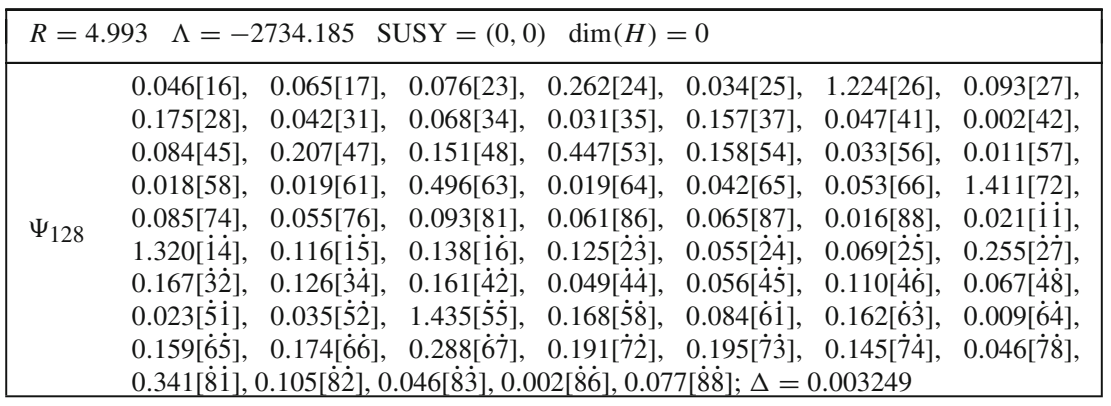




\begin{tabular}{|c|c|}
\hline $\mathcal{M}_{s} / g^{2}$ & $\begin{array}{l}-3709.038,-3383.450,-2985.036,-2621.756,-1915.641,-1458.258,-851.672, \\
-710.027,-360.683,-274.519,-101.445,-0.036,-0.035,-0.034,-0.033, \\
-0.029,-0.028,-0.027,-0.025,-0.023,-0.021,-0.020,-0.017(\times 2),-0.015, \\
-0.013,-0.012,-0.011,-0.010(\times 2),-0.009,-0.008,-0.007,-0.006, \\
-0.005(\times 2),-0.003,-0.002,0.000(\times 4), 0.001,0.002(\times 2), 0.003(\times 2), 0.004(\times 2), \\
0.006,0.007,0.009,0.010,0.011,0.013(\times 3), 0.015,0.018,0.019,0.020,0.021, \\
0.024,0.025,0.027,0.028,0.030,576.718,1480.111,2238.282,2464.567, \\
2696.496,3207.514,3776.598,4617.058,5304.732,5461.952,5857.965,6479.092, \\
6756.775,8142.389,8658.673,8725.730,9526.228,9715.372,10774.833,10872.396, \\
11208.154,11651.297,12185.661,12234.783,12833.043,12869.577,13588.627, \\
13647.343,13756.395,13911.020,14569.223,14824.411,14982.795,15797.546, \\
16019.139,16204.362,16399.798,16584.501,17311.918,17473.114,17670.906, \\
17808.456,18023.684,18182.002,19560.405,19595.444,20332.592,20739.479, \\
21099.066,22191.970,22488.638,23813.910,24055.209,25967.903,26072.100, \\
27964.156,27972.906,29489.018,29646.552,35262.129,35362.669\end{array}$ \\
\hline $\mathcal{M}_{v} / g$ & $\begin{array}{l}-155.096,-154.132,-147.453,-141.839,-140.637,-136.365,-132.886, \\
-131.312,-129.053,-125.511,-123.468,-119.004,-116.668,-114.471, \\
-110.294,-108.578,-102.520,-100.493,-98.955,-95.424,-94.979,-86.578, \\
-83.384,-74.463,-67.790,-58.004,-42.770,-28.202,0.000(\times 72), 28.202, \\
42.770,58.004,67.790,74.463,83.384,86.578,94.979,95.424,98.955,100.493, \\
102.520,108.578,110.294,114.471,116.668,119.004,123.468,125.511,129.053, \\
131.312,132.886,136.365,140.637,141.839,147.453,154.132,155.096\end{array}$ \\
\hline$A_{1}$ & $\begin{array}{l}-109.573,-109.027,-103.074,-98.935,-86.379,-78.971,-70.822,-57.886 \text {, } \\
57.886,70.822,78.971,86.379,98.935,103.074,109.027,109.573\end{array}$ \\
\hline$A_{3}$ & $\begin{array}{l}-328.720,-327.082,-309.223,-296.805,-259.138,-236.913,-212.465, \\
-177.986,-177.298,-174.163,-173.658,-173.524,-171.216,-169.098, \\
-164.594,-160.856,-155.729,-154.788,-149.627,-148.829,-146.351, \\
-144.960,-143.183,-142.384,-141.222,-139.795,-136.858,-135.016, \\
-133.404,-132.086,-129.461,-128.422,-126.385,-125.365,-124.030, \\
-123.076,-121.186,-118.863,-117.950,-115.416,-112.477,-111.130, \\
-109.615,-105.656,-102.619,-98.363,-94.769,-92.164,-90.561,-87.910, \\
-86.690,-80.840,-77.415,-75.070,-65.761,-63.287,-55.846,-51.815, \\
-47.099,-41.770,-31.327,-21.941,-13.653,-2.343,2.343,13.653,21.941, \\
31.327,41.770,47.099,51.815,55.846,63.287,65.761,75.070,77.415,80.840, \\
86.691,87.910,90.561,92.164,94.769,98.363,102.619,105.656,109.615,111.130, \\
112.477,115.416,117.950,118.863,121.186,123.076,124.030,125.365,126.385, \\
128.422,129.461,132.086,133.404,135.016,136.858,139.795,141.222,142.384, \\
143.183,144.960,146.351,148.829,149.627,154.788,155.729,160.857,164.594, \\
169.098,171.216,173.524,173.658,174.163,177.298,177.986,212.465,236.913, \\
259.138,296.804,309.223,327.082,328.720\end{array}$ \\
\hline
\end{tabular}

\begin{tabular}{|c|c|c|c|c|c|c|}
\hline \multicolumn{7}{|c|}{$R=4.995 \quad \Lambda=-2811.104 \quad \operatorname{SUSY}=(0,0) \quad \operatorname{dim}(H)=0$} \\
\hline$\Psi_{128}$ & $\begin{array}{l}0.026[12], \\
0.024[35], \\
0.052[52], \\
0.027[63], \\
0.109[77], \\
0.196[\dot{1} \dot{6}], \\
0.043[\dot{3} \dot{4}], \\
0.040[\dot{5} \dot{4}], \\
0.001[\dot{7} \dot{7}],\end{array}$ & $\begin{array}{cc}0.139[13], & 0.007[16], \\
0.010[36], & 0.007[37], \\
0.032[53], & 0.010[55], \\
0.050[65], & 0.047[67], \\
0.019[78], & 0.050[82], \\
0.040[\dot{1} \overline{7}], & 0.032[\dot{2} \dot{2}], \\
0.024[\dot{3} \dot{8}], & 0.149[\dot{4} \dot{3}], \\
0.001[\dot{5} \dot{6}], & 0.086[\dot{6} \dot{1}], \\
0.140[\dot{7} \dot{8}], & 0.005[\dot{8} \dot{1}], 0.0 \\
\end{array}$ & $\begin{array}{l}1.411[17], \\
0.092[38], \\
1.411[56], \\
1.371[71], \\
0.236[83], \\
0.467[\dot{2} \dot{4}], \\
0.140[\dot{4} \dot{5}], \\
0.033[\dot{6} \dot{2}], \\
14[\dot{8} \dot{7}] ; \Delta=\end{array}$ & $\begin{array}{l}0.236[25], \\
0.006[43], \\
0.018[57], \\
0.031[73], \\
0.024[86], \\
0.036[\dot{2} \overline{7}], \\
0.025[\dot{4} \dot{8}], \\
0.011[\dot{6} \dot{6}], \\
0.003376\end{array}$ & $\begin{array}{l}0.139[27], \\
0.052[44], \\
0.019[61], \\
0.321[75], \\
0.043[1 \dot{1}], \\
0.265[\dot{2} \dot{8}] \\
0.014[\dot{5} \dot{2}] \\
0.196[\dot{7} \dot{4}] \\
\end{array}$ & $\begin{array}{l}0.005[32], \\
0.021[48], \\
0.021[62], \\
0.010[76], \\
0.003[\dot{1} \dot{3}], \\
0.005[\dot{3} \dot{2}], \\
0.173[\dot{5} \dot{3}], \\
1.584[\dot{7} \dot{6}],\end{array}$ \\
\hline
\end{tabular}




\begin{tabular}{|c|c|}
\hline $\mathcal{M}_{s} / g^{2}$ & $\begin{array}{l}-5039.683,-3749.860,-3352.973,-2647.356,-1938.490,-1446.393,-992.780, \\
-339.008,-260.596,-192.833,-176.426,-75.899,-0.042,-0.040,-0.038, \\
-0.034,-0.033,-0.030,-0.029,-0.028,-0.026,-0.024,-0.022,-0.019, \\
-0.018,-0.017,-0.016,-0.015,-0.013(\times 2),-0.011,-0.010,-0.009,-0.008, \\
-0.007,-0.005(\times 2),-0.004,-0.002,-0.001,0.000(\times 2), 0.002,0.004,0.005(\times 2), \\
0.006,0.008,0.009,0.010,0.011,0.012,0.014(\times 2), 0.015,0.017,0.018,0.020,0.024, \\
0.025,0.028,0.029,0.031,0.033,0.034,0.035,0.041,0.043,877.461,1066.267, \\
2227.991,2666.852,3431.989,4045.593,4470.486,5581.739,6092.585,6156.372, \\
7131.244,7455.249,8553.094,8737.456,9101.221,9431.477,9723.090,10087.859, \\
10297.142,11097.379,11477.601,12194.392,12305.792,13106.496,13462.497, \\
13679.509,14161.126,14310.198,14622.293,14804.038,15675.250,15838.510, \\
16139.352,17958.599,18110.641,18552.148,18962.918,19042.309,19865.471, \\
20224.376,21940.177,22174.493,23008.092,23023.079,24994.843,25452.402, \\
26505.727,26580.132,27406.293,27413.969,28044.488,28980.695,32389.334, \\
32674.352,35581.261,35599.925,38351.605,38386.480,40762.021,40828.552\end{array}$ \\
\hline $\mathcal{M}_{v / g}$ & $\begin{array}{l}-174.320,-174.089,-164.954,-164.595,-151.647,-150.901,-140.822, \\
-138.710,-128.619,-125.935,-123.353,-122.556,-115.114,-113.863, \\
-112.267,-109.736,-104.575,-103.341,-98.433,-97.079,-94.224,-87.766, \\
-85.663,-82.611,-73.072,-59.323,-53.455,-12.786,0.000(\times 72), 12.786, \\
53.455,59.323,73.072,82.611,85.663,87.766,94.224,97.079,98.434,103.341, \\
104.575,109.736,112.267,113.863,115.114,122.556,123.353,125.935,128.619, \\
138.710,140.822,150.901,151.647,164.595,164.954,174.089,174.320\end{array}$ \\
\hline$A_{1}$ & $\begin{array}{l}-120.437,-118.514,-108.668,-105.893,-88.490,-86.350,-60.456,-55.550, \\
55.550,60.456,86.350,88.490,105.893,108.668,118.514,120.437\end{array}$ \\
\hline$A_{3}$ & $\begin{array}{l}-361.311,-355.542,-326.005,-317.678,-265.469,-259.050,-201.709, \\
-201.576,-196.971,-196.743,-182.742,-182.567,-181.369,-171.748, \\
-170.557,-166.649,-166.066,-165.893,-162.757,-161.287,-156.823, \\
-155.483,-153.252,-153.096,-150.063,-149.269,-144.938,-144.498, \\
-142.185,-139.348,-138.667,-136.052,-131.907,-131.036,-128.783, \\
-126.759,-125.264,-119.931,-119.361,-116.691,-114.878,-114.161, \\
-111.720,-108.234,-106.108,-103.067,-96.296,-94.080,-89.006,-87.291, \\
-85.554,-81.842,-74.137,-69.959,-65.151,-55.643,-51.585,-46.732, \\
-42.849,-37.648,-35.324,-20.076,-14.401,-4.159,4.159,14.401,20.076, \\
35.325,37.648,42.849,46.732,51.585,55.643,65.151,69.959,74.137,81.842, \\
85.554,87.291,89.006,94.080,96.297,103.067,106.108,108.234,111.720,114.161, \\
114.878,116.691,119.361,119.931,125.264,126.759,128.783,131.036,131.907, \\
136.052,138.667,139.348,142.185,144.498,144.938,149.269,150.063,153.096, \\
153.252,155.483,156.823,161.287,162.757,165.893,166.066,166.649,170.557, \\
171.748,181.369,182.568,182.742,196.743,196.971,201.576,201.709,259.050,\end{array}$ \\
\hline
\end{tabular}

\begin{tabular}{|c|c|c|c|c|c|c|c|}
\hline \multicolumn{8}{|c|}{$R=5.019 \quad \Lambda=-2829.083 \quad \operatorname{SUSY}=(0,0) \quad \operatorname{dim}(H)=0$} \\
\hline$\Psi_{128}$ & $\begin{array}{l}1.671[12], \\
0.381[24], \\
0.051[42], \\
0.014[62], \\
0.015[82], \\
0.053[\dot{1} \dot{3}], \\
0.065[\dot{2} \dot{8}], \\
1.303[\dot{4} \dot{6}], \\
0.032[\dot{6} \dot{3}], \\
0.047[\overline{\dot{4}}],\end{array}$ & $\begin{array}{l}0.005[13], \\
0.021[31], \\
0.122[47], \\
0.093[64], \\
0.018[83], \\
0.033[\dot{1} \dot{5}], \\
0.026[\dot{3} \dot{4}], \\
0.066[\dot{4} \dot{7}], \\
0.119[\dot{6} \dot{4}], \\
.004[\dot{8} \dot{3}], 0\end{array}$ & $\begin{array}{l}0.057[14], \\
0.024[32], \\
0.084[51], \\
0.080[65], \\
0.048[84], \\
0.152[\dot{1} \dot{6}], \\
0.021[\dot{3} \dot{5}], \\
0.001[\dot{5} \dot{3}], \\
0.036[\dot{6} \dot{5}], \\
083[\dot{8} \dot{6}] ; \Delta\end{array}$ & $\begin{array}{l}0.009[17], \\
0.091[33], \\
0.010[52], \\
0.025[68], \\
0.009[86], \\
0.010[\dot{2} \dot{3}], \\
0.068[\dot{3} \dot{7}], \\
0.090[\dot{5} \dot{6}], \\
0.160[\dot{6} \dot{6}], \\
=0.003162\end{array}$ & $\begin{array}{l}0.040[18], \\
0.437[34], \\
0.080[54], \\
0.076[76], \\
0.003[87], \\
0.059[\dot{2} \dot{4}], \\
0.066[\dot{3} \dot{8}], \\
0.036[\dot{5} \dot{8}], \\
0.020[\dot{7} \dot{1}],\end{array}$ & $\begin{array}{l}0.066[21], \\
0.156[36], \\
0.046[56], \\
0.029[78], \\
0.217[\dot{1} \dot{1}], \\
1.706[\dot{2} \dot{5}], \\
0.013[\dot{4} \dot{1}], \\
0.788[\dot{6} \dot{1}], \\
0.027[\dot{7} \dot{2}],\end{array}$ & $\begin{array}{l}0.261[23], \\
0.423[41], \\
0.264[61], \\
0.024[81], \\
0.047[\dot{1} \dot{2}], \\
0.055[\dot{2} \dot{6}], \\
0.085[\dot{4} \dot{4}], \\
0.186[\dot{6} \dot{2}], \\
1.708[\dot{7} \dot{3}],\end{array}$ \\
\hline
\end{tabular}




\begin{tabular}{|c|c|}
\hline $\mathcal{M}_{s} / g^{2}$ & $\begin{array}{l}-4134.500,-4048.976,-2781.433,-2740.228,-2167.664,-1233.249,-907.381, \\
-406.397,-217.272,-79.983,-0.048,-0.043,-0.037,-0.036,-0.034,-0.031, \\
-0.029,-0.027,-0.026,-0.025,-0.022(\times 2),-0.020,-0.018,-0.017,-0.016, \\
-0.015,-0.014,-0.013,-0.011,-0.010(\times 2),-0.009,-0.008,-0.007,-0.006, \\
-0.003,-0.002(\times 2), 0.000(\times 3), 0.002,0.003,0.005,0.006,0.007,0.008(\times 2), \\
0.009,0.012,0.013,0.014,0.017,0.018,0.020,0.021,0.024,0.025,0.026,0.027, \\
0.029,0.033,0.037,0.040,0.044,526.298,691.901,1840.718,2493.910,3144.446, \\
3905.941,4839.343,5185.538,5262.086,6635.187,7215.429,7618.435,7936.676, \\
8148.664,9029.418,9754.017,9934.365,10302.343,10372.103,11045.128, \\
11931.509,12132.455,12459.086,13143.344,13366.178,13525.616,13755.159, \\
14131.703,14397.039,15567.448,15618.081,16447.815,16464.286,17025.959, \\
17122.270,17504.262,19526.877,19826.302,22110.936,22203.073,22569.352, \\
22644.357,25064.119,25105.678,25255.872,25424.361,27277.949,27361.250, \\
31493.211,31516.611,33460.929,33501.981,34946.806,34953.110,45313.035, \\
45316.702,46493.592,46493.907,66741.287,66742.622,73037.425,73037.966\end{array}$ \\
\hline $\mathcal{M}_{v} / g$ & $\begin{array}{l}-215.997,-215.992,-186.235,-186.212,-165.846,-165.779,-146.730, \\
-146.126,-139.174,-137.949,-129.014,-127.120,-123.142,-121.308, \\
-117.186,-111.998,-107.884,-106.245,-104.419,-101.317,-99.075, \\
-94.716,-88.530,-86.869,-71.077,-70.047,-29.609,-15.356,0.000(\times 72), \\
15.356,29.609,70.047,71.077,86.869,88.530,94.716,99.075,101.317,104.419, \\
106.245,107.884,111.998,117.186,121.308,123.142,127.120,129.014,137.949, \\
139.174,146.126,146.730,165.779,165.846,186.212,186.235,215.992,215.997\end{array}$ \\
\hline$A_{1}$ & $\begin{array}{l}-129.946,-129.156,-117.955,-117.590,-94.519,-89.615,-70.828,-61.505, \\
61.505,70.828,89.615,94.519,117.590,117.955,129.156,129.946\end{array}$ \\
\hline$A_{3}$ & $\begin{array}{l}-389.838,-387.467,-353.866,-352.771,-283.556,-268.845,-268.456, \\
-268.455,-223.911,-223.905,-215.932,-215.913,-212.484,-198.828, \\
-198.795,-184.516,-183.148,-183.117,-176.538,-176.484,-174.244, \\
-174.199,-161.376,-161.154,-154.114,-153.526,-150.654,-148.514, \\
-147.115,-143.338,-140.239,-137.480,-135.156,-134.183,-130.133, \\
-127.589,-125.567,-124.898,-122.656,-120.170,-118.723,-117.177, \\
-114.658,-112.948,-112.194,-108.594,-102.959,-98.360,-96.975,-91.080, \\
-83.701,-82.806,-80.776,-73.854,-72.236,-67.223,-53.679,-53.197, \\
-45.003,-41.536,-34.890,-25.494,-13.800,-10.871,10.871,13.800,25.494, \\
34.890,41.536,45.003,53.197,53.679,67.223,72.236,73.854,80.776,82.806, \\
83.701,91.080,96.975,98.360,102.959,108.594,112.194,112.948,114.658, \\
117.177,118.723,120.170,122.656,124.898,125.567,127.589,130.133,134.183, \\
135.156,137.480,140.239,143.338,147.115,148.514,150.654,153.526,154.114, \\
161.154,161.376,174.198,174.244,176.484,176.538,183.117,183.148,184.516, \\
198.795,198.828,212.484,215.913,215.932,223.905,223.911,268.455,268.456, \\
268.845,283.556,352.771,353.866,387.467,389.838\end{array}$ \\
\hline
\end{tabular}

\begin{tabular}{|c|c|c|c|c|c|c|c|}
\hline \multicolumn{8}{|c|}{$R=5.020 \quad \Lambda=-2841.660 \quad \operatorname{SUSY}=(0,0) \quad \operatorname{dim}(H)=0$} \\
\hline$\Psi_{128}$ & $\begin{array}{l}0.177[11], \\
1.489[34], \\
0.040[53], \\
0.328[72], \\
0.031[86], \\
0.049[\dot{2} \dot{4}], \\
0.084[\dot{4} \dot{3}], \\
0.066[\dot{5} \dot{4}], \\
1.204[\dot{7} \dot{1}], \\
0.063[\dot{8} \dot{ },\end{array}$ & $\begin{array}{l}0.050[14], \\
0.045[35], \\
0.146[56], \\
0.002[74], \\
0.028[88], \\
0.057[\dot{2} \dot{5}], \\
0.214[\dot{4} \dot{4}], \\
1.179[\dot{5} \dot{7}], \\
0.038[\overline{7} \dot{2}], \\
0.021[\dot{8} \dot{6}], 0 .\end{array}$ & $\begin{array}{c}0.059[15], \\
0.034[37], \\
1.174[62], \\
1.208[75], \\
0.042[\dot{1} \dot{2}], \\
0.159[\dot{2} \dot{8}], \\
0.182[\dot{4} \dot{5}], \\
0.046[\dot{6} \dot{1}], \\
0.112[\dot{7} \dot{4}], \\
143[\dot{8} 7], 0.0\end{array}$ & $\begin{array}{c}0.318[17], \\
0.348[45], \\
0.054[64], \\
0.318[76], \\
0.161[\dot{1} \dot{4}], \\
0.053[\dot{3} \dot{1}], \\
0.088[\dot{4} \dot{6}], \\
0.158[\dot{6} \dot{3}], \\
0.164[\dot{7} \dot{5}], \\
88[\dot{8} \dot{8}] ; \Delta=\end{array}$ & $\begin{array}{l}0.219[21], \\
0.178[46], \\
0.281[65], \\
0.016[78], \\
0.141[\dot{1} \dot{5}], \\
0.064[\dot{3} \dot{2}], \\
0.010[\dot{4} \overline{7}], \\
1.437[\dot{6} \dot{4}], \\
0.145[\dot{7} \dot{6}], \\
0.003219\end{array}$ & $\begin{array}{l}0.030[24], \\
0.057[48], \\
0.022[67], \\
0.255[81], \\
0.076[\dot{1} \dot{6}], \\
0.128[\dot{3} \dot{4}], \\
0.071[\dot{5} \dot{1}], \\
0.109[\dot{6} \dot{7}], \\
1.522[\dot{8} \dot{2}],\end{array}$ & $\begin{array}{l}0.110[31], \\
0.021[52], \\
0.091[71], \\
0.026[85], \\
0.095[\dot{1} \dot{8}], \\
0.327[\dot{3} \dot{5}], \\
0.016[\dot{5} \dot{2}], \\
0.219[\dot{6} \dot{8}], \\
0.083[\dot{8} \dot{3}],\end{array}$ \\
\hline
\end{tabular}




\begin{tabular}{|c|c|}
\hline $\mathcal{M}_{s} / g^{2}$ & $\begin{array}{l}-4268.539,-3604.183,-2806.376,-2755.472,-2202.375,-1733.434,-758.685, \\
-331.854,-144.862,-51.994,-0.045,-0.036,-0.033,-0.030,-0.029,-0.026, \\
-0.024,-0.022,-0.020(\times 2),-0.017,-0.015,-0.014,-0.012,-0.011,-0.009, \\
-0.008(\times 2),-0.007,-0.005,-0.003,-0.002,0.000(\times 2), 0.002(\times 2), 0.003,0.004, \\
0.005,0.007(\times 3), 0.009,0.010,0.011,0.012,0.013,0.014,0.015,0.016,0.017,0.019, \\
0.021(\times 2), 0.023,0.025,0.029,0.031,0.036,0.038,0.040,0.041,0.042,0.046, \\
0.053,0.058,496.847,579.435,1355.514,1924.301,2222.250,2553.012,3517.005, \\
4135.860,4297.135,4461.139,4977.909,5557.505,6899.856,8071.686,8421.033, \\
9061.049,9847.212,9996.417,11372.641,11512.105,12023.327,12383.681, \\
12410.690,12963.400,13257.514,13493.081,13881.585,14256.935,15024.637, \\
15113.202,15573.292,16044.568,16186.599,17156.122,17188.990,17595.387, \\
17696.658,17814.385,18372.579,19724.041,20039.098,20405.432,20426.554, \\
21137.243,22153.610,22430.012,23080.486,23137.134,23996.020,24842.491, \\
25989.810,26221.916,28552.666,28573.196,33681.519,33749.307,35630.243, \\
35654.890,44131.757,44154.038,53515.115,53517.192\end{array}$ \\
\hline $\mathcal{M}_{v} / g$ & $\begin{array}{l}-187.845,-187.825,-163.937,-163.664,-149.838,-149.499,-141.008, \\
-139.533,-130.794,-128.657,-124.292,-121.843,-120.213,-116.866, \\
-111.063,-110.777,-106.481,-103.610,-101.205,-97.009,-95.788,-89.396, \\
-84.675,-79.389,-75.187,-62.346,-56.655,-21.262,0.000(\times 72), 21.262, \\
56.655,62.346,75.187,79.389,84.675,89.396,95.788,97.010,101.205,103.610, \\
106.481,110.777,111.063,116.866,120.213,121.843,124.292,128.657,130.794, \\
139.533,141.008,149.499,149.838,163.664,163.938,187.825,187.845\end{array}$ \\
\hline$A_{1}$ & $\begin{array}{l}-123.194,-121.512,-108.998,-106.021,-88.133,-78.248,-73.552,-60.334, \\
60.334,73.552,78.248,88.133,106.021,108.998,121.512,123.194\end{array}$ \\
\hline$A_{3}$ & $\begin{array}{l}-369.581,-364.536,-326.995,-318.062,-264.399,-234.745,-224.700, \\
-224.690,-220.656,-204.905,-204.895,-181.744,-181.691,-181.002, \\
-176.382,-176.201,-166.282,-164.749,-161.433,-160.241,-157.377, \\
-155.349,-151.400,-150.104,-148.353,-146.558,-144.042,-142.343, \\
-141.784,-140.305,-136.396,-134.821,-132.135,-130.292,-128.886, \\
-125.394,-124.824,-122.959,-120.249,-119.711,-117.142,-114.281, \\
-112.142,-109.794,-108.639,-107.638,-100.235,-98.613,-94.031,-91.600, \\
-88.147,-80.874,-76.534,-72.710,-67.479,-63.599,-55.134,-51.351, \\
-43.735,-42.984,-27.444,-25.775,-16.167,-10.430,10.430,16.167,25.775, \\
27.444,42.984,43.735,51.351,55.135,63.599,67.479,72.709,76.534,80.874, \\
88.147,91.600,94.031,98.613,100.235,107.638,108.639,109.794,112.142, \\
114.281,117.142,119.711,120.249,122.959,124.824,125.394,128.887,130.292, \\
132.135,134.821,136.396,140.305,141.784,142.343,144.042,146.558,148.353, \\
150.104,151.400,155.349,157.377,160.241,161.433,164.749,166.282,176.201, \\
176.382,181.002,181.691,181.744,204.895,204.905,220.656,224.690,224.700, \\
234.746,264.399,318.062,326.995,364.536,369.582\end{array}$ \\
\hline
\end{tabular}

\begin{tabular}{|c|c|c|c|c|c|c|c|}
\hline \multicolumn{8}{|c|}{$R=5.142 \quad \Lambda=-3396.918 \quad \operatorname{SUSY}=(0,0) \quad \operatorname{dim}(H)=0$} \\
\hline$\Psi_{128}$ & $\begin{array}{l}0.162[12], \\
1.242[26], \\
0.138[43], \\
0.016[64], \\
0.192[84], \\
0.227[\dot{2} 7], \\
0.337[\dot{4} \dot{2}], \\
0.409[\dot{5} \dot{4}], \\
0.002[\dot{7} \dot{1}], \\
0.015[\dot{8} \dot{3}],\end{array}$ & $\begin{array}{l}0.074[16], \\
0.095[27], \\
0.007[45], \\
0.192[65], \\
0.040[88], \\
0.024[\dot{2} \dot{8}], \\
1.439[\dot{4} \dot{3}], \\
0.017[\dot{5} \dot{5}], \\
0.211[\dot{7} \dot{3}], \\
1.099[\dot{8} \dot{4}], 0 .\end{array}$ & $\begin{array}{c}1.312[17], \\
0.011[31], \\
0.007[52], \\
0.142[71], \\
0.031[\dot{1} \dot{4}], \\
1.429[\dot{3} \dot{1}], \\
0.060[\dot{4} \dot{4}], \\
0.119[\dot{5} \dot{7}], \\
0.230[\dot{7} \dot{5}], \\
148[\dot{8} \dot{5}], 0.1\end{array}$ & $\begin{array}{c}0.094[18], \\
0.140[34], \\
0.027[53], \\
0.073[73], \\
0.183[\dot{1} \dot{5}], \\
0.172[\dot{3} \dot{2}], \\
0.194[\dot{4} \dot{7}], \\
1.476[\dot{5} \dot{8}], \\
0.270[\dot{7} \dot{6}], \\
22[\dot{8} \dot{6}] ; \Delta=\end{array}$ & $\begin{array}{l}0.062[21], \\
0.054[38], \\
0.015[57], \\
0.146[75], \\
0.413[\dot{1} \dot{6}], \\
0.175[\dot{3} \dot{5}], \\
0.019[\dot{4} \dot{8}], \\
0.191[\dot{6} \dot{2}], \\
0.079[\dot{7} \dot{7}], \\
0.003234\end{array}$ & $\begin{array}{l}0.020[22], \\
0.050[41], \\
0.041[61], \\
0.063[77], \\
0.010[\dot{1} \dot{8}], \\
0.026[\dot{3} \dot{8}], \\
0.060[\dot{5} \dot{1}], \\
0.027[\dot{6} \dot{4}], \\
0.272[\dot{7} \dot{8}],\end{array}$ & $\begin{array}{l}0.121[23], \\
1.553[42], \\
0.023[62], \\
0.037[82], \\
0.945[\dot{2} \dot{5}], \\
0.011[\dot{4} \dot{1}], \\
0.038[\dot{5} \dot{2}], \\
0.037[\dot{6} \dot{8}], \\
0.085[\dot{8} \dot{2}],\end{array}$ \\
\hline
\end{tabular}




\begin{tabular}{|c|c|}
\hline $\mathcal{M}_{s} / g^{2}$ & $\begin{array}{l}-6433.262,-5176.533,-3445.873,-1776.621,-1629.741,-1138.285,-922.826, \\
-606.640,-321.012,-79.665,-0.073,-0.063,-0.059,-0.052,-0.050,-0.048, \\
-0.040,-0.039,-0.037,-0.035,-0.031(\times 2),-0.027,-0.025,-0.024,-0.023, \\
-0.016(\times 2),-0.015,-0.013,-0.010(\times 2),-0.008,-0.006,-0.005,-0.004, \\
-0.003,0.000(\times 2), 0.002,0.003,0.005,0.007,0.008,0.011,0.013,0.015,0.018(\times 2), \\
0.020,0.021,0.024,0.027,0.029,0.031,0.032,0.038,0.040,0.042,0.043,0.048, \\
0.052,0.054,0.058,0.063,0.079,215.743,745.287,2428.459,2886.346,3380.364, \\
4536.355,5678.256,6148.932,6401.515,6685.298,8786.417,9708.706,9745.625, \\
10213.156,11649.006,11803.592,12182.892,12317.915,12489.505,14062.233, \\
14334.841,14996.367,15184.792,15542.605,15838.614,16098.712,17474.847, \\
17943.936,18661.639,18788.989,18875.137,20168.450,21439.528,22266.736, \\
22831.007,23920.036,23960.720,24239.662,26103.793,26405.328,26843.914, \\
27566.096,29788.460,30039.073,30409.858,30449.904,34526.427,34780.043, \\
35928.605,36015.901,38057.753,38138.826,47536.443,47567.372,50889.987, \\
50907.585,53927.225,53939.318,56202.736,56218.447,89093.658,89094.188\end{array}$ \\
\hline $\mathcal{M}_{v} / g$ & $\begin{array}{l}-226.383,-226.367,-197.403,-197.298,-190.525,-190.277,-159.534, \\
-159.146,-149.608,-147.262,-139.519,-137.982,-135.354,-132.695, \\
-130.768,-129.191,-116.057,-114.930,-111.063,-109.435,-102.440, \\
-101.879,-98.597,-96.897,-89.261,-82.268,-24.512,-20.933,0.000(\times 72), \\
20.933,24.512,82.268,89.261,96.897,98.597,101.879,102.440,109.435,111.064, \\
114.930,116.057,129.191,130.768,132.695,135.354,137.982,139.519,147.262, \\
149.608,159.146,159.534,190.277,190.525,197.298,197.403,226.367,226.383\end{array}$ \\
\hline$A_{1}$ & $\begin{array}{l}-149.680,-148.914,-109.152,-108.019,-104.553,-100.100,-94.253, \\
-69.540,69.540,94.253,100.100,104.553,108.019,109.152,148.914,149.680\end{array}$ \\
\hline$A_{3}$ & $\begin{array}{l}-449.041,-446.742,-327.456,-324.058,-313.660,-300.301,-282.758, \\
-271.969,-271.968,-260.315,-260.313,-231.744,-231.726,-208.620, \\
-206.906,-206.866,-203.026,-202.840,-191.500,-191.195,-176.297, \\
-175.890,-175.575,-174.905,-167.660,-166.677,-163.057,-161.897, \\
-152.493,-151.443,-150.404,-149.987,-149.132,-146.581,-145.711, \\
-142.104,-140.616,-139.800,-138.052,-135.589,-134.676,-132.395, \\
-127.456,-126.498,-122.754,-112.683,-108.201,-107.512,-106.835, \\
-102.990,-95.914,-95.286,-89.958,-86.949,-82.859,-80.497,-75.993, \\
-63.206,-59.028,-52.382,-42.608,-38.258,-14.711,-14.395,14.395,14.711, \\
38.258,42.608,52.382,59.028,63.206,75.993,80.497,82.859,86.949,89.958, \\
95.286,95.914,102.990,106.835,107.512,108.201,112.683,122.754,126.498, \\
127.456,132.395,134.676,135.589,138.052,139.800,140.616,142.104,145.711, \\
146.581,149.132,149.987,150.404,151.443,152.493,161.897,163.057,166.677, \\
167.660,174.905,175.575,175.890,176.297,191.195,191.500,202.840,203.027, \\
206.866,206.906,208.620,231.726,231.744,260.313,260.315,271.968,271.969, \\
282.758,300.301,313.660,324.058,327.456,446.742,449.041\end{array}$ \\
\hline
\end{tabular}

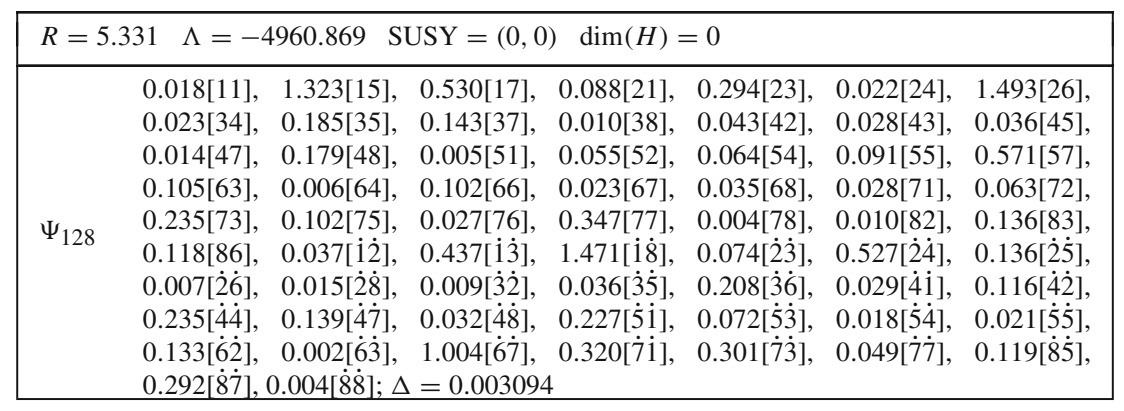




\begin{tabular}{|c|c|}
\hline $\mathcal{M}_{s} / g^{2}$ & $\begin{array}{l}-8810.346,-6449.877,-4496.062,-2975.418,-2718.911,-2399.747,-333.848, \\
-209.471,-204.707,-0.080,-0.067,-0.064,-0.059,-0.056,-0.051,-0.048, \\
-0.042,-0.041,-0.037,-0.035,-0.033,-0.031,-0.029,-0.026,-0.022, \\
-0.020,-0.019,-0.015,-0.014,-0.011,-0.010,-0.008,-0.004,-0.001,0.000, \\
0.001,0.004,0.006,0.007,0.010,0.013,0.015,0.017,0.019,0.022,0.024,0.026, \\
0.030,0.032,0.033,0.035,0.038,0.042,0.045,0.047,0.050,0.054,0.055,0.059, \\
0.062,0.070,0.074,0.078,0.083,0.097,3486.283,3850.802,6560.444,7006.250, \\
9136.863,10447.842,11504.284,13359.753,13789.798,13995.127,14709.531, \\
15669.740,17038.556,18288.488,20795.525,20888.196,22436.330,23839.836, \\
23881.869,24656.405,24824.328,25275.553,25732.779,27275.166,27386.658, \\
28902.364,29358.659,30274.118,30751.800,31187.331,31842.622,32967.086, \\
34502.341,35696.506,36219.619,36329.933,36523.489,38574.258,40055.828, \\
41417.592,41488.255,42246.729,42600.712,43977.358,45968.272,47102.505, \\
48510.240,49464.413,53211.561,55172.068,56373.259,56938.668,58286.611, \\
61879.646,63920.013,64158.201,65414.763,66558.044,67705.508,77573.641, \\
77707.847,85685.393,85907.714\end{array}$ \\
\hline $\mathcal{M}_{v} / g$ & $\begin{array}{l}-242.380,-240.981,-229.548,-223.792,-216.574,-209.404,-201.894, \\
-195.281,-194.943,-190.290,-186.352,-180.383,-178.496,-171.882, \\
-168.262,-161.005,-154.366,-150.680,-149.860,-146.426,-138.176, \\
-135.258,-132.529,-123.969,-117.462,-102.155,-87.733,-23.684, \\
0.000(\times 72), 23.684,87.732,102.155,117.462,123.969,132.529,135.258,138.176, \\
146.426,149.860,150.679,154.366,161.005,168.262,171.882,178.496,180.383, \\
186.352,190.290,194.944,195.281,201.894,209.404,216.573,223.793,229.548, \\
240.981,242.380\end{array}$ \\
\hline$A_{1}$ & $\begin{array}{l}-165.818,-165.118,-147.763,-143.876,-133.864,-126.622,-106.549, \\
-102.728,102.728,106.549,126.622,133.864,143.876,147.763,165.118,165.818\end{array}$ \\
\hline$A_{3}$ & 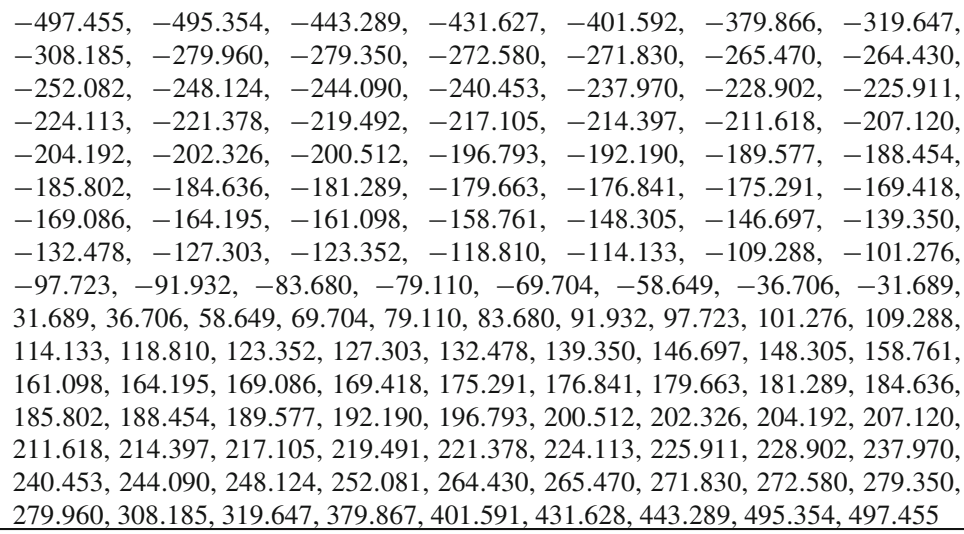 \\
\hline
\end{tabular}

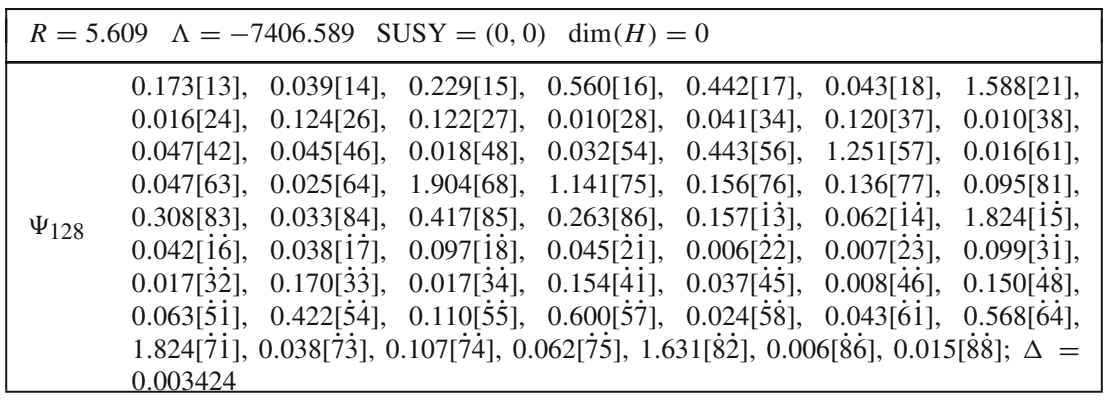




\begin{tabular}{|c|c|}
\hline $\mathcal{M}_{s} / g^{2}$ & $\begin{array}{l}-9689.180,-7685.877,-7638.058,-4100.767,-3507.540,-3153.466,-701.825, \\
-94.954,-0.166,-0.153,-0.145,-0.126,-0.119,-0.112,-0.104,-0.095, \\
-0.089,-0.082,-0.074,-0.073,-0.069,-0.065,-0.060,-0.055,-0.044, \\
-0.036,-0.032,-0.031,-0.026,-0.021,-0.020,-0.015,-0.013,-0.011, \\
-0.008,0.000,0.002,0.008,0.010,0.012,0.022,0.025,0.032(\times 2), 0.036,0.039, \\
0.042,0.048,0.054,0.060,0.064,0.069,0.071,0.075,0.079,0.091,0.097,0.100, \\
0.104,0.117,0.129,0.132,0.153,0.162,5626.585,6053.271,12531.016,17576.186, \\
18009.460,18191.415,18323.749,21213.443,23147.451,25640.471,25841.429, \\
28979.529,29485.247,32238.844,33045.791,34668.176,34886.375,37823.197, \\
39675.477,39893.631,42164.601,43106.417,43363.531,45166.701,46083.753, \\
47535.657,48904.263,51714.756,56749.144,56905.337,59629.715,60216.218, \\
61401.045,62729.230,64336.908,64956.964,67262.858,67760.333,70021.839, \\
71168.827,76406.717,76464.085,79530.680,79865.171,82468.501,83083.874, \\
83558.779,85587.688,89619.527,90255.920,90773.552,92422.611,95996.777, \\
98614.953,100118.557,104206.972,114203.436,115285.705,127664.722, \\
127821.613,161488.523,161517.547,164160.223,164164.320\end{array}$ \\
\hline $\mathcal{M}_{v} / g$ & 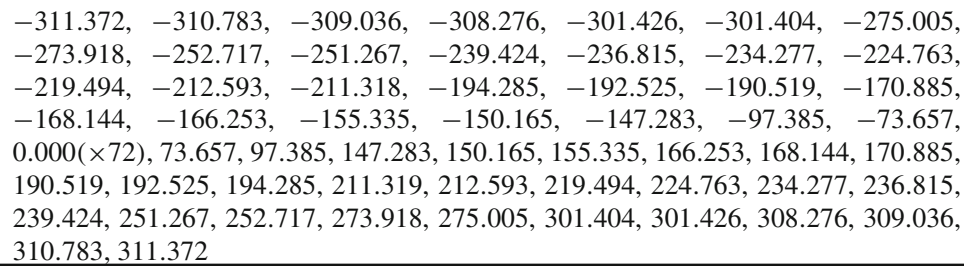 \\
\hline$A_{1}$ & $\begin{array}{l}-214.928,-209.264,-205.890,-196.824,-171.540,-171.430,-149.599, \\
-118.578,118.578,149.599,171.430,171.540,196.824,205.890,209.264,214.928\end{array}$ \\
\hline$A_{3}$ & 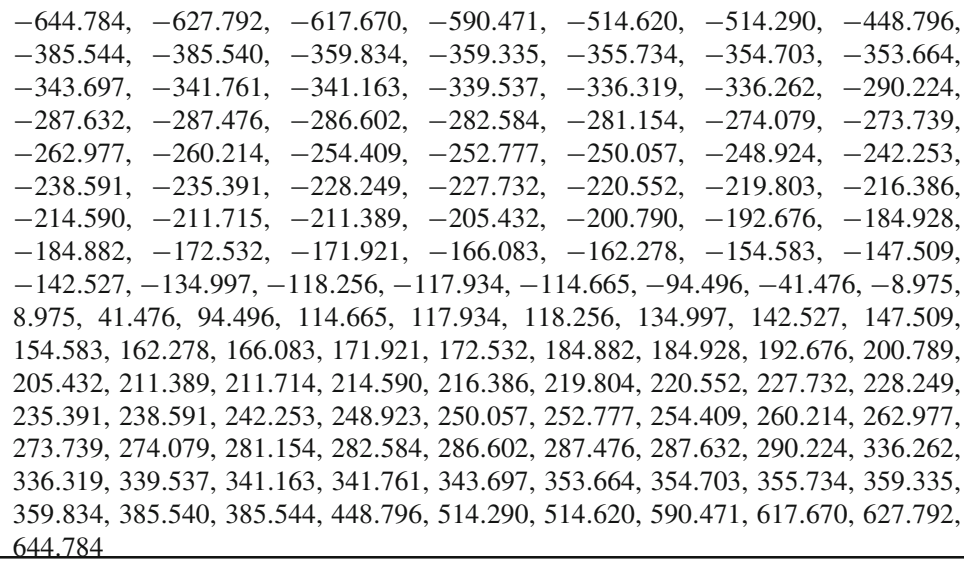 \\
\hline
\end{tabular}

\section{Conclusion and outlook}

The utilization of sensitivity analysis backpropagation for the numerical study of the scalar potentials of extended supergravity models appears to be a dramatically successful technique for finding and determining some of the properties of their vacua. A detailed analysis of the one model with the technically most challenging scalar potential known reveals that the number of possible vacuum solutions is quite large, much larger than the number of solutions that have been found hitherto. While some computationally helpful special properties of Spin(16) $\Gamma$-matrices simplify the problem in 
$D=3$, it is expected that these techniques will turn out to be equally powerful for the analysis of the $D=4$ and 5 potentials which involve the smaller exceptional groups $E_{6}$ and $E_{7}$. Detailed information about the properties of novel vacua of these models should be of considerable value in situations where the $\mathrm{AdS}_{4} / \mathrm{CFT}_{3}$ and $\mathrm{AdS}_{5} / \mathrm{CFT}_{4}$ correspondences can be utilized to obtain new insights. While a detailed analysis of the symmetry breaking structure of all dimensionally reduced models of maximal supergravity (including a large variety of noncompact gauge groups) may be within technological reach now, special attention should hence be paid to these particular cases.

As the number of stationary points of the scalar potential of $S O(8)$-gauged $D=4$, $\mathcal{N}=8$ supergravity is expected to be considerably smaller than that of the maximal three-dimensional model analyzed here, and as it is technically far easier to work with densely occupied complex $56 \times 56$ matrices than with real $248 \times 248$ matrices, the most sensible strategy to approach this particular problem seems to be to aim for very high numerical precision (i.e. hundreds of digits) right from the beginning so that conjectures for analytic expressions for the locations and properties of stationary points can be generated semi-automatically [15], to serve as important guidance for stringent proofs establishing the existence of new solutions.

Acknowledgments It is a pleasure to thank Hermann Nicolai, Maria Alessandra Papa, and Steffen Grunewald both for hospitality and access to supercomputing resources at the Albert Einstein Institute where part of this work was done. Also, I benefitted from discussions with Andreas Griewank, Shaun Forth, and Andy Keane on automatic differentiation as well as optimization.

\section{References}

1. Bagger, J., Lambert, N.: Gauge symmetry and supersymmetry of multiple M2-branes. Phys. Rev. D. 77, 065008 (2008). [arXiv:0711.0955 [hep-th]]

2. Berestetsky, V.B., Lifshitz, E.M., Pitaevsky, L.P.: Quantum Electrodynamics, Pergamon Press, Oxford (1982) 652 P. (Course Of Theoretical Physics, 4)

3. Bergshoeff, E.A., Hohm, O., Nutma, T.A.: A note on E11 and three-dimensional gauged supergravity. JHEP 0805, 081 (2008). [arXiv:0803.2989 [hep-th]]

4. Bergshoeff, E.A., Hohm, O., Kleinschmidt, A., Nicolai, H., Nutma, T.A., Palmkvist, J.: E10 and gauged maximal supergravity, arXiv:0810.5767 [hep-th]

5. Benna, M., Klebanov, I., Klose, T., Smedback, M.: Superconformal Chern-Simons theories and $\mathrm{AdS}_{4} / \mathrm{CFT}_{3}$ correspondence. JHEP 0809, 072 (2008). [arXiv:0806.1519 [hep-th]]

6. Breitenlohner, P., Freedman, D.Z.: Stability in gauged extended supergravity. Ann. Phys. 144, 249 (1982)

7. de Buyl, S., Henneaux, M., Paulot, L.: Extended E(8) invariance of 11-dimensional supergravity. JHEP 0602, 056 (2006). [arXiv:hep-th/0512292]

8. Byrd, R.H., Lu, P., Nocedal, J.: A limited memory algorithm for bound constrained optimization. SIAM J. Sci. Stat. Comput. 16(5), 1190-1208 (1995)

9. Cremmer, E., Julia, B., Scherk, J.: Supergravity theory in 11 dimensions. Phys. Lett. B 76, 409 (1978)

10. Cremmer, E., Julia, B., Lu, H., Pope, C.N.: Dualisation of dualities. I. Nucl. Phys. B 523, 73 (1998). [arXiv:hep-th/9710119]

11. Damour, T., Henneaux, M., Nicolai, H.: E(10) and a 'small tension expansion' of M-theory. Phys. Rev. Lett. 89, 221601 (2002). [arXiv:hep-th/0207267]

12. Englert, F., Houart, L., Taormina, A., West, P.C.: The symmetry of M-theories. JHEP 0309, 020 (2003). [arXiv:hep-th/0304206]

13. Fischbacher, T.: Introducing LambdaTensor1.0: a package for explicit symbolic and numeric Lie algebra and Lie group calculations. [arXiv:hep-th/0208218] 
14. Fischbacher, T., Nicolai, H., Samtleben, H.: Vacua of maximal gauged $D=3$ supergravities. Class. Quant. Gravit. 19, 5297 (2002). [arXiv:hep-th/0207206]

15. Fischbacher, T.: New vacua of $\mathrm{SO}(8)$ gauged $N=8 \mathrm{D}=4$ supergravity (in preparation)

16. Granlund, T.: The GNU multiple precision arithmetic library. http://gmplib.org

17. Green, M.B., Schwarz, J.H., Witten, E.: Superstring Theory: Introduction (Cambridge Monographs On Mathematical Physics), vol. 1, p. 469. Cambridge University Press, London (1987)

18. Griewank, A.: On automatic differentiation. In: Iri, M., Tanabe, K. (eds.) Mathematical Programming: Recent Developments and Applications. Kluwer Academic Publishers, Dordrecht, pp. 83-108

19. Griewank, A., Juedes, D., Utke, J.: A package for the automatic differentiation of algorithms written in C/C++. ACM TOMS 22(2), 131-167 (1996). (Algor. 755)

20. Gustavsson, A.: Algebraic structures on parallel M2-branes. arXiv:0709.1260 [hep-th]

21. Higgs, P.: Broken symmetries and the masses of gauge bosons. Phys. Rev. Lett. 0031-9007 13(16), 508 (1964)

22. Hull, C.M., Warner, N.P.: The potentials of the gauged $N=8$ supergravity theories. Nucl. Phys. B 253, 675 (1985)

23. Klebanov, I., Klose, T., Murugan, A.: $\mathrm{AdS}_{4} / \mathrm{CFT}_{3}$ — squashed, stretched and warped. arXiv:0809.3773 [hep-th]

24. Maldacena, J.M.: The large N limit of superconformal field theories and supergravity. Adv. Theor. Math. Phys. 2231 (1998). [Int. J. Theor. Phys. 38, 1113 (1999)] [arXiv:hep-th/9711200]

25. MATLAB is a registered trademark by The MathWorks. http://www.mathworks.com

26. Moler, C., Van Loan, C.: Nineteen Dubious Ways to Compute the Exponential of a Matrix, Twenty-Five Years Later. SIAM Rev. ISSN 0036-1445 45(1), 3-50 (2003)

27. Nicolai, H., Samtleben, H.: Compact and noncompact gauged maximal supergravities in three dimensions. JHEP 0104, 022 (2001). [arXiv:hep-th/0103032]

28. Pearlmutter, B.A., Siskind, J.M.: Using programming language theory to make automatic differentiation sound and efficient. Lecture Notes in Computational Science and Engineering, Advances in Automatic Differentiation, ISSN 1439-7358, vol. 64, pp. 79-90 (2008). doi:10.1007/ 978-3-540-68942-3_8

29. Eric Jones, Travis Oliphant, Pearu Peterson and others. SciPy: Open Source Scientific Tools for Python. http://www.scipy.org (2001)

30. Seiberg, N., Witten, E.: Electric - magnetic duality, monopole condensation, and confinement in $N=2$ supersymmetric Yang-Mills theory. Nucl. Phys. B 426, 19 (1994). [erratum-ibid. B 430, 485 (1994)] [arXiv:hep-th/9407087]

31. Speelpenning, B.: Compiling fast Partial Derivatives of Functions given by Algorithms, Ph.D. Dissertation, Department of Computer Science, University of Illinois at Urbana-Champaign (1980)

32. Warner, N.P.: Some properties of the scalar potential in gauged supergravity theories. Nucl. Phys. B 231, 250 (1984)

33. Warner, N.P.: Some new extrema of the scalar potential of gauged $N=8$ supergravity. Phys. Lett. B 128, 169 (1983)

34. Weinberg, S.: A model of leptons. Phys. Rev. Lett. 19, 1264 (1967)

35. Weinberg, S.: The Quantum Theory of Fields, vol. 1, p. 609. Foundations. Cambridge, University Press, London (1995)

36. Witten, E.: Quantum field theory and the Jones polynomial. Commun. Math. Phys. 121, 351 (1989)

37. Witten, E.: String theory dynamics in various dimensions. Nucl. Phys. B 443, 85 (1995). [arXiv:hep-th/ 9503124] 